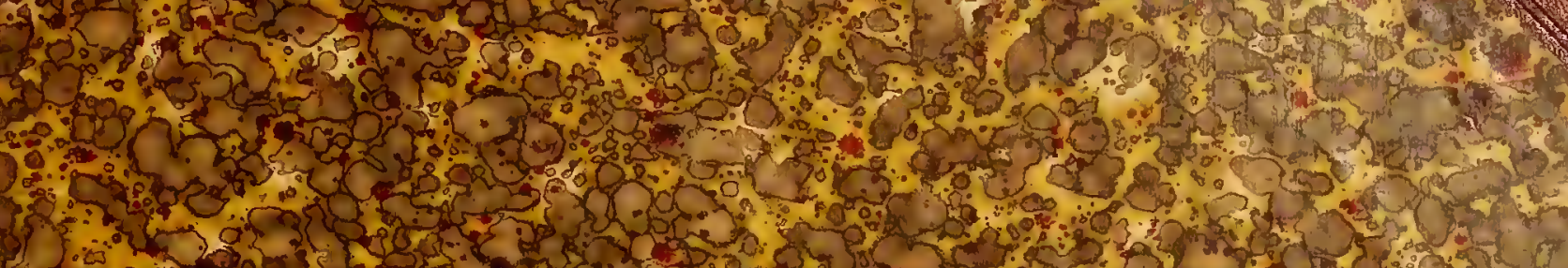

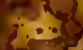

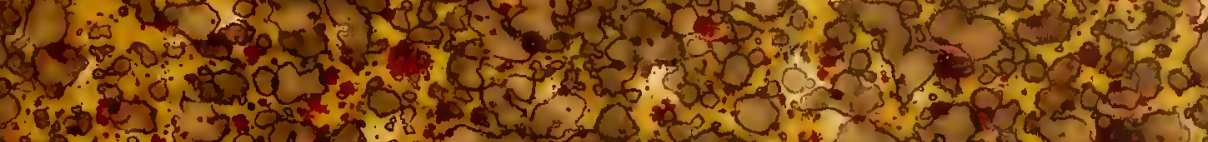

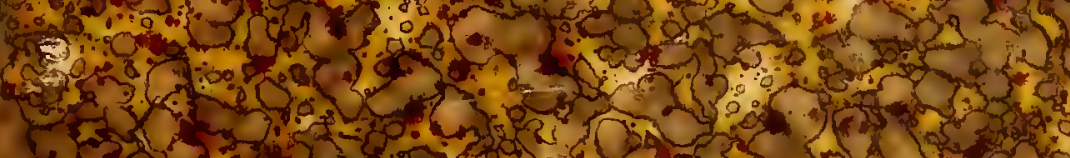

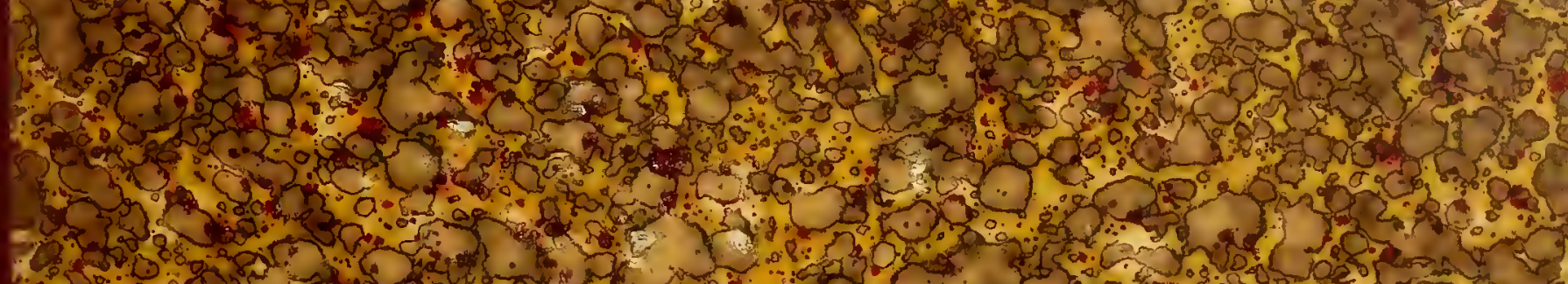

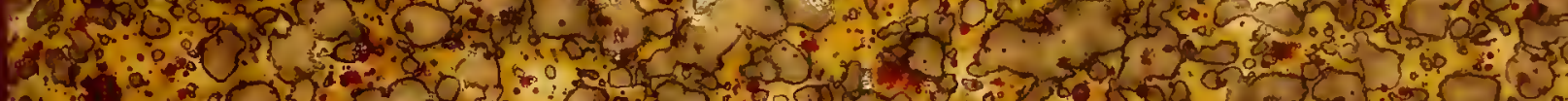

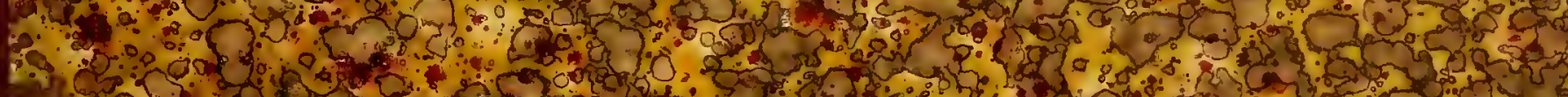

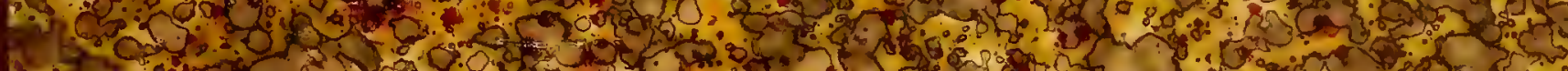

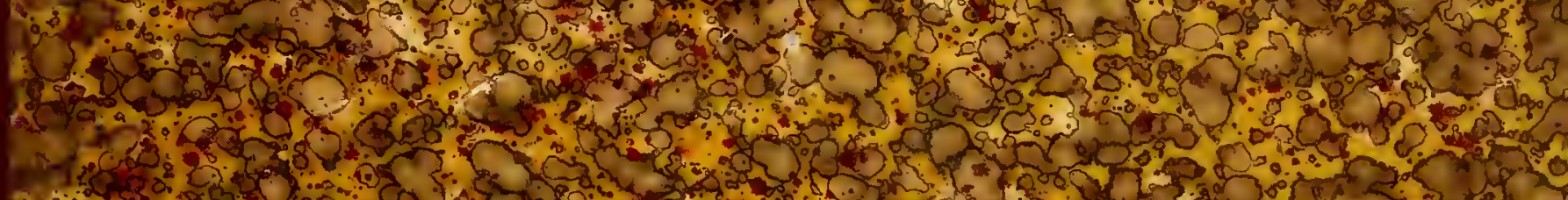

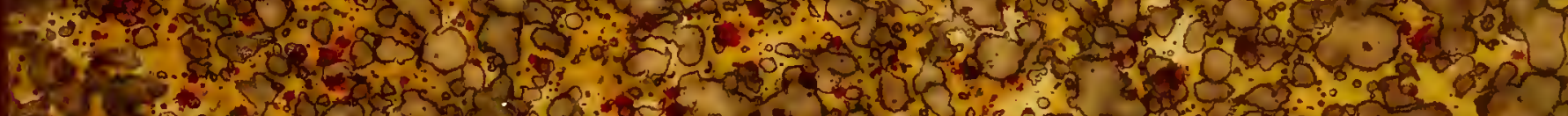

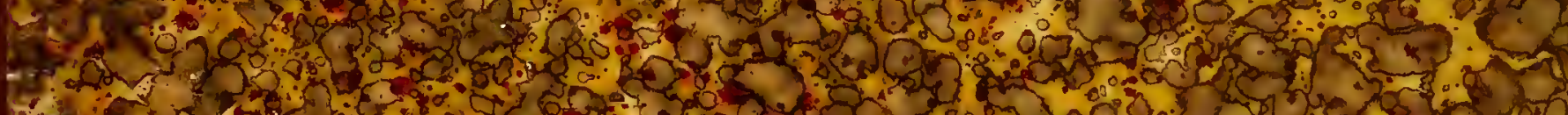

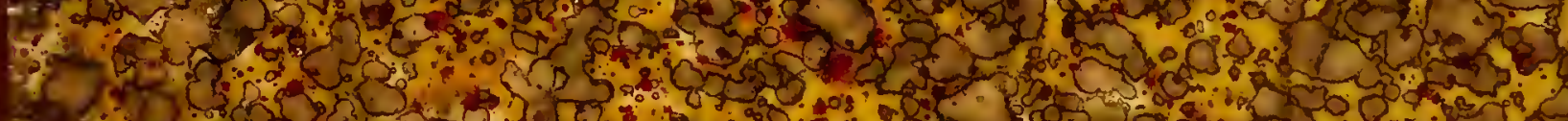

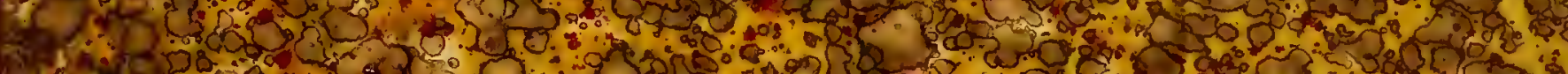

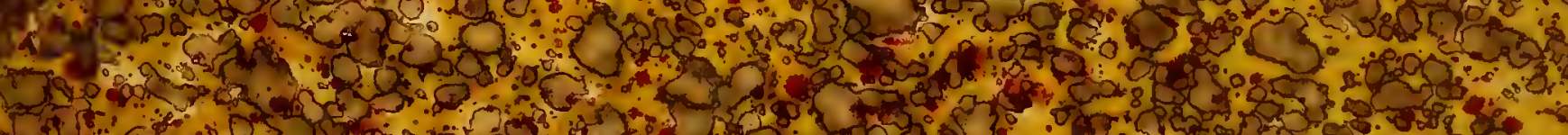

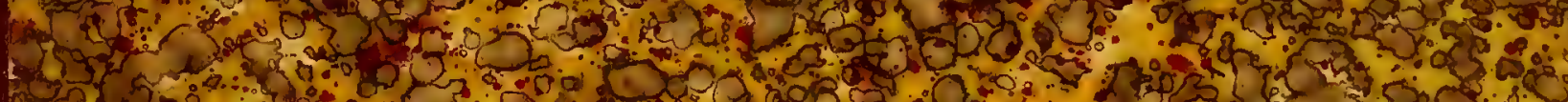

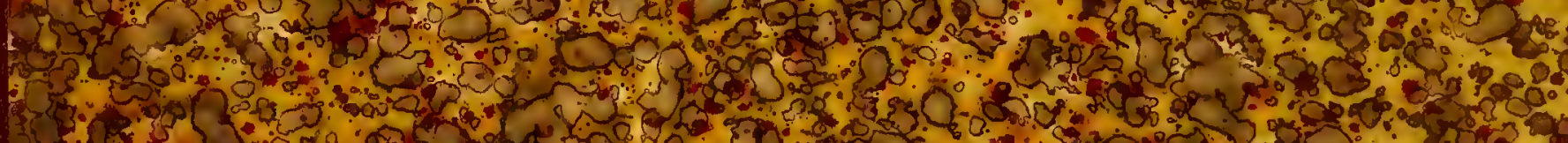
1. 3 .

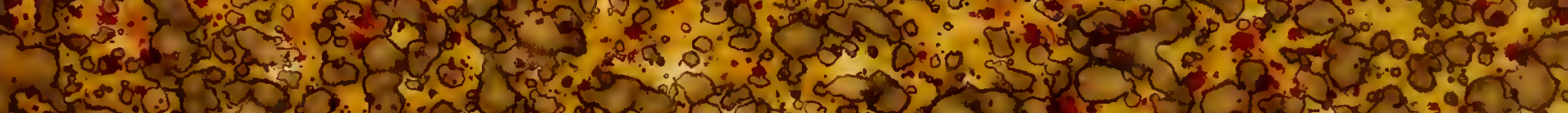

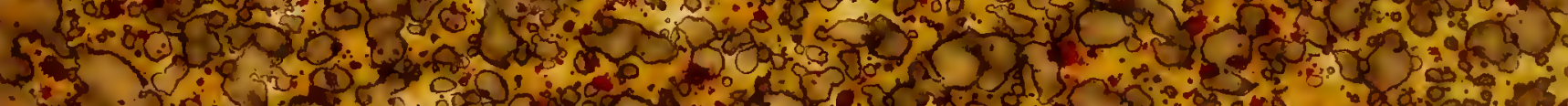

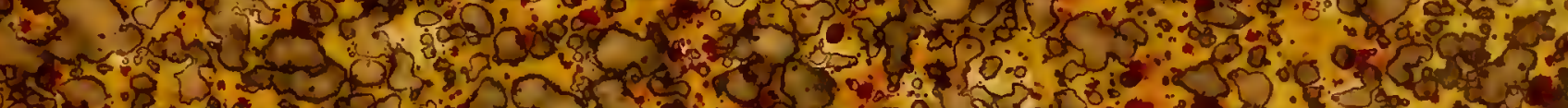

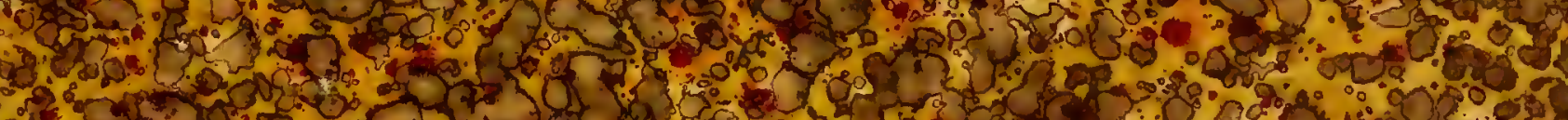

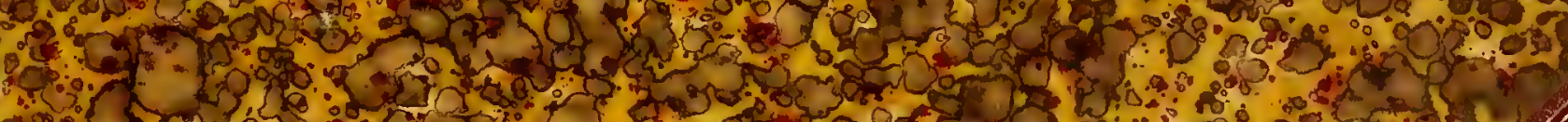

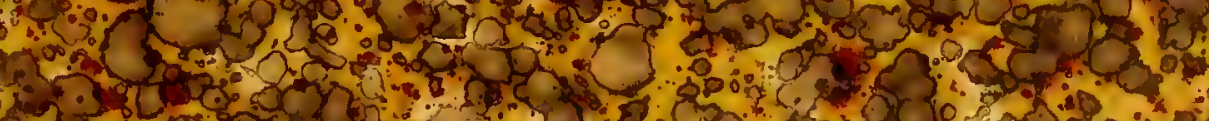

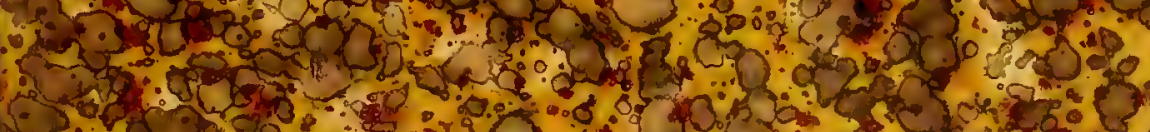

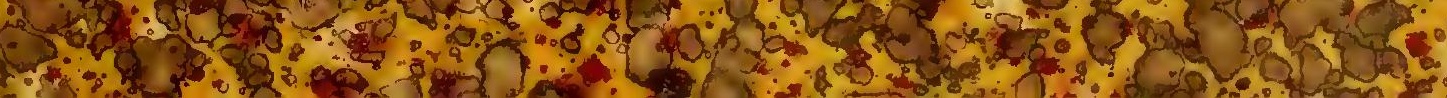




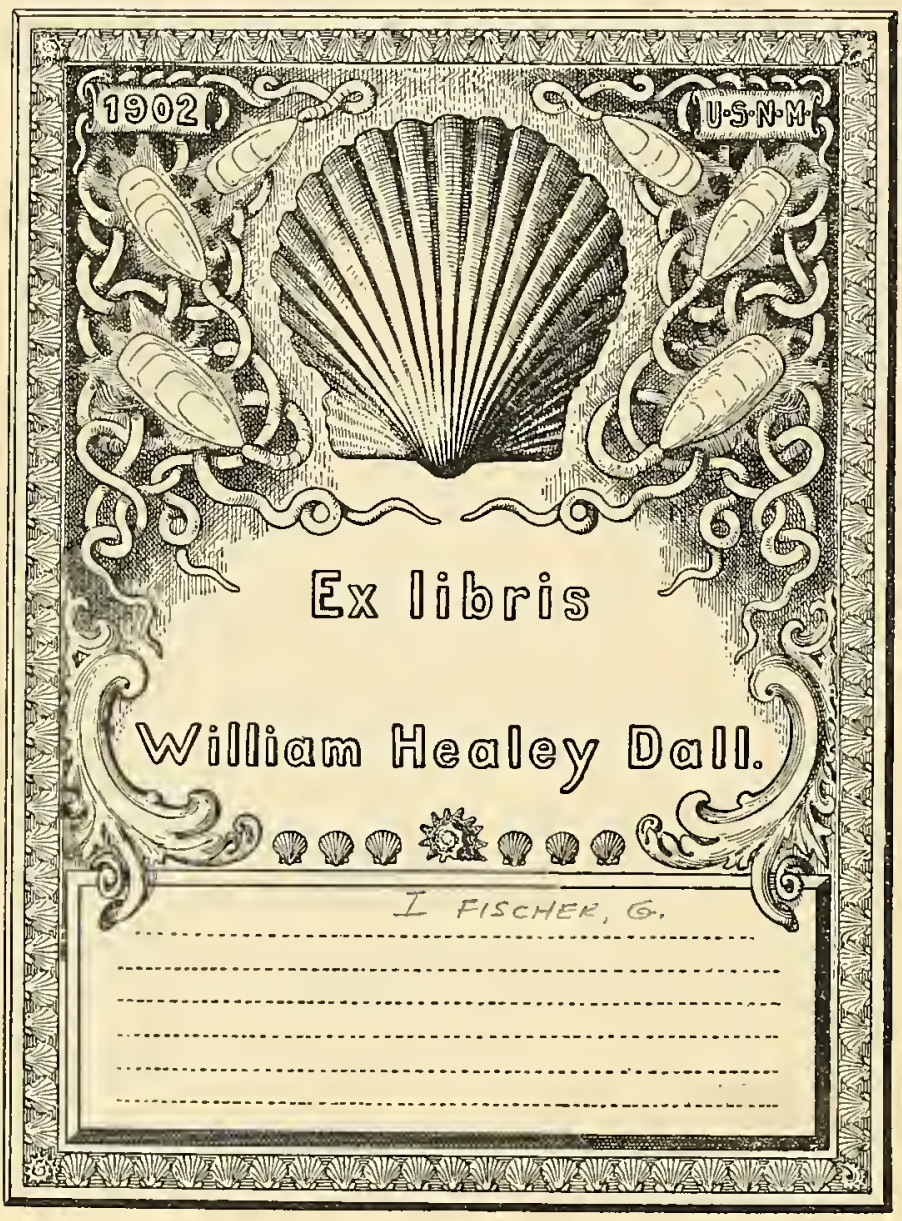




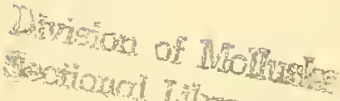
(n) 


G. F I S C H E R I

TABULAE SYNOPTICAE

Z O O G $N$ O S I A E

IN USUM AUDITORUM EDITAE.

C UM TABULIS AENEIS.

MOSQUA E. 1808. 
r.

, 


\title{
TABLEAUX SYNOPTIQUES
}

$D E$

\section{$\mathrm{Z} O O O G \quad \mathrm{~N} O \mathrm{~S} I \mathrm{E}$.}

publit́s À L'USAge de SES ÉLÈves À L'UNiversité IMPÉRIALE DE MOSCOU

par le Professeur G. Fischer.

avec fix planches.

Division of Molineks

Westional tibrosty

\begin{abstract}
à M O S C O U
de l'Imprimerie de l'Université Impériale.

1808.
\end{abstract}


Avec permission du. bureau de cenfure éłabli pour l'arrondiffement de l'Université Impériale de Moscon.

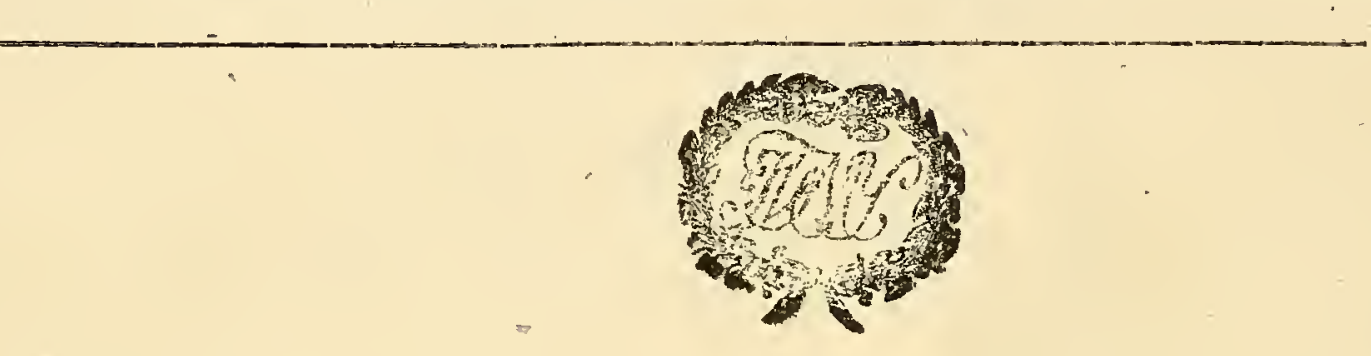




\section{MONSIEUR LE COMTE}

\section{ALEXIS D RAZOUMOFSKY,}

Conseifler privé actuel, Chambellan actuel, Curateur de l'Uniyersité Impériale de Moscou, Président de ia Société Impériale des Naturalistes, membre de plusieuris sociétés savantes, Chevalier de l'Ordre de St. Alexandre Neesiry, etco etc. 
A qui pourrois-je mieux adrefler le défir que j’ai d'être utile à mes élèves, fi ce n'éft à Votre Excellence, qui' s'intéreffe fi vivement aux progrès de l'inftruction publique; comme rien ne facilite autant l'étude d'une fcience quelconque que la réduction de fes principes en tableaux.

J'ai préféré ne m'occuper dans les tableaux de Zoognosie que j'ai l'honneur de Vous présenter et qui font d'une grande étendue, que des généralités et laisfer les détails à la démonftration, afin que l'oeil puiffe tout à la fois embraffer une claffe entière ou du moins une de les principales divifions. L'avantage que j'ai vu refulter de ces tableaux pendant plufieurs cours á. l'Université durant lesquels je les ai communiqué en manufcrits à mes élèves, promet le meilleur fuccès à la forme que je leur ai donné.

C'eft à la demande de quelques ans de mes élèves que j’ai ajouté des détails fur les Mollusques. Votre Excellence en connoit la belle collection 
donnée par Mr. de Bemidoff; elle efr.placée de forte qu'elle peut être étudiée à l'aide de ma délcription fans aucun autre lécours. J'abrégerai cette partie dans mes cours.

Je m'eftimerai très heureux fi mon onvrage acquiert l'approbation de Votre Excellence dont les connoilfances dans les fciences, naturelles ainf que dans l'hiftoire-de leurs progrès actuels me font attacher un grand prix a fon jugement.

Agréez, je Vous prie, l'allurance du profond refpect et de la haute efime avec lesquels je fuis

\section{DE VOTRE EXGELLENCE}

Moscou le

15 Nov, 1808 . le très humble et très obéissant ferviteur

Gotthelf Fischer. 


\section{T $A U L A E S L^{\circ} \mathbb{N} O P T I C A E$ \\ Z O O G NOS I A F \\ IN USUM DISCENTIUM EDITAE。}




\section{TA B U LAANIMAIIU}

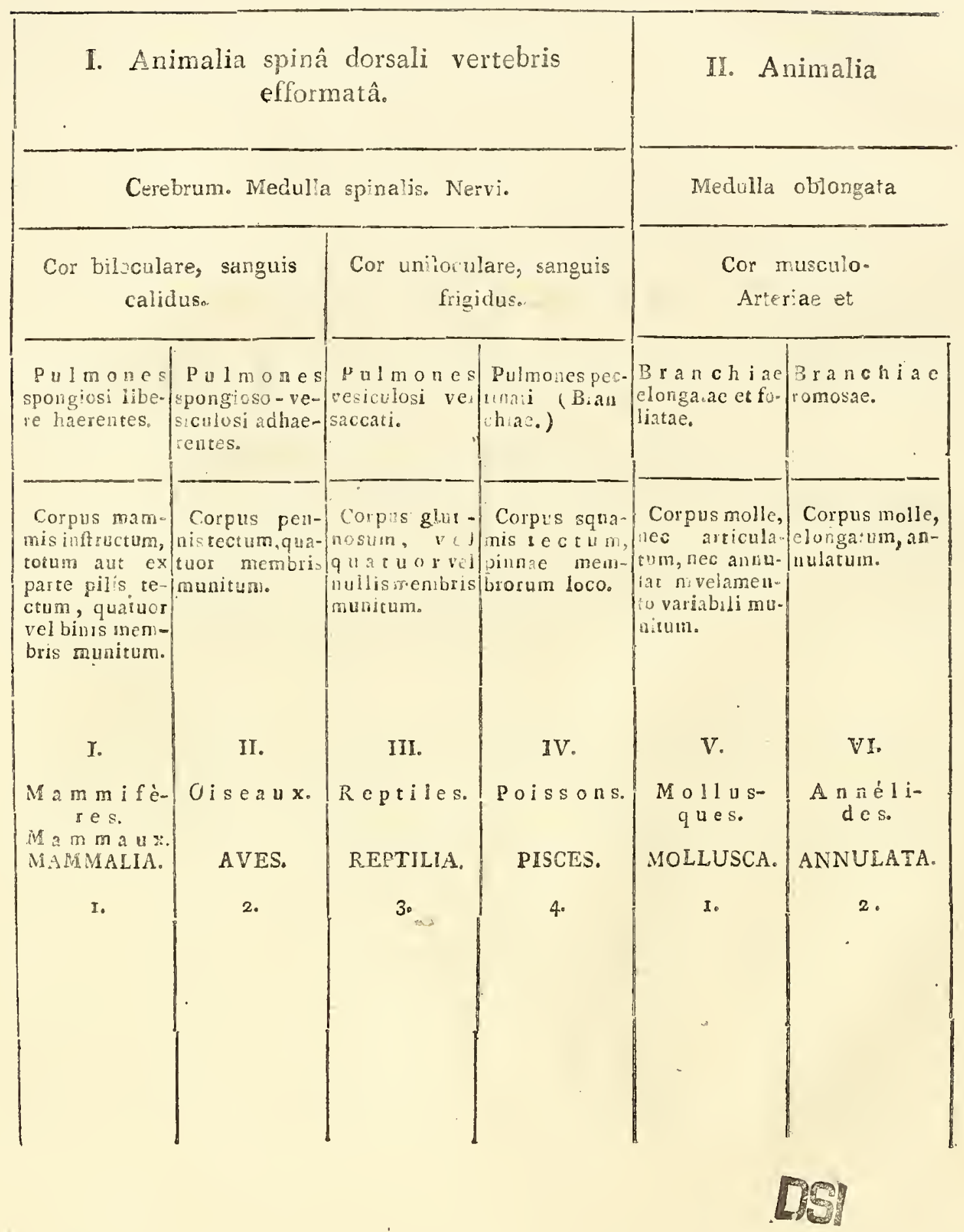




\section{CLASSES E U M E R A S.}

vertebris destituta.

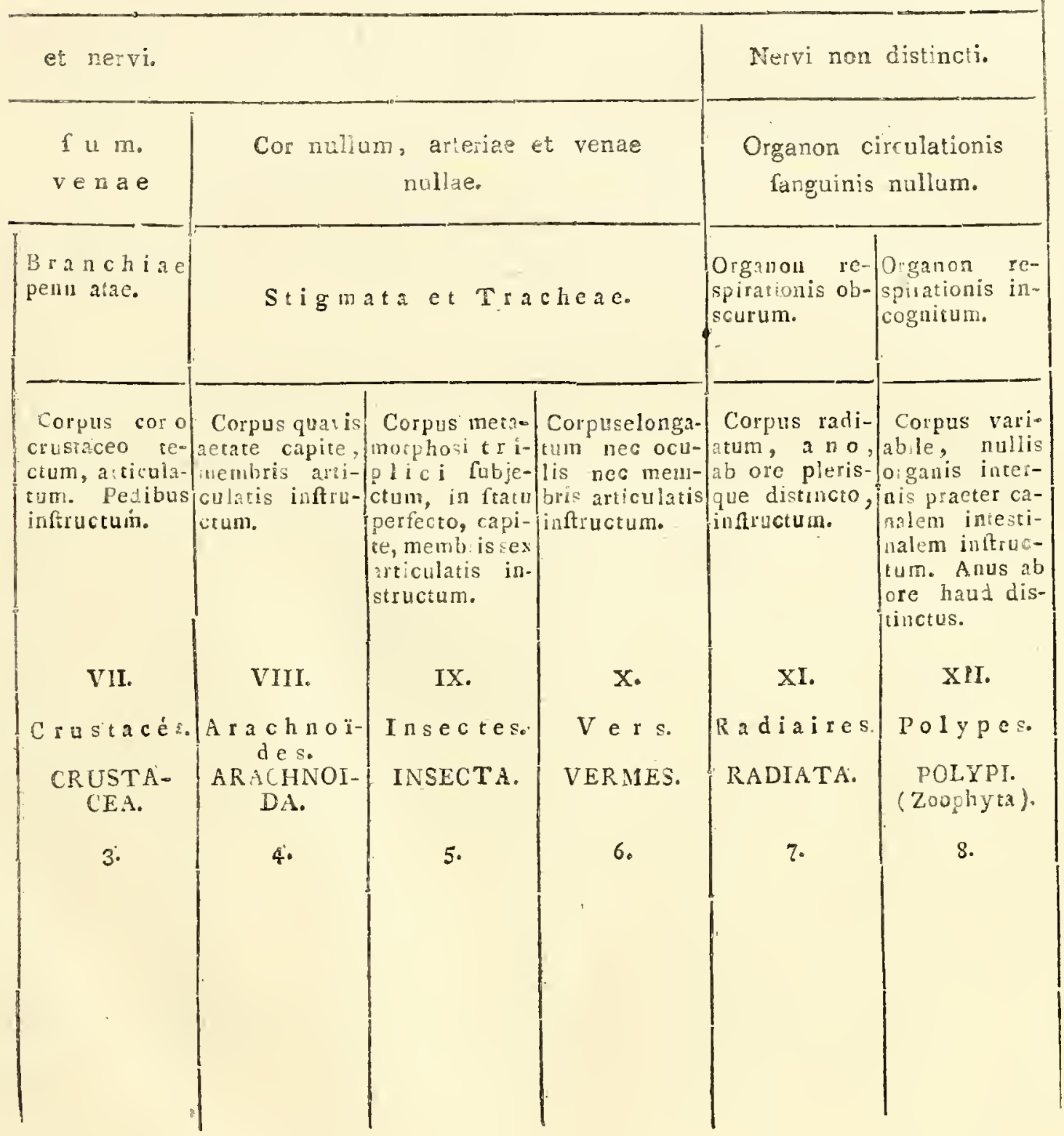




\section{I. $C L A S S I S$.}

$M$ a $m$ m a $l$ i a.

A. Qnatuor pedibus aut ecrum analogis instructa.

Tetrapodes. Quadrupèdes. Quadrupede.

I. Pedibus aut digitis membranâ non cinctis.

Fis s ipèdes. Fissipeda.

a. Digitis unguiculatis.

Onguiculés. Digités. Unguiculata. Digitata.

b. digitis ungulatis.

Ongulés. Ungziculata.

II. Pedibus aut digitis membranâ cinctis.

N e x o p è des. Nectopodes. Nectopodir.

a. Membranâ volatui inserviente.

P.t.e.ropodeso Pteropoda.

* Extremitates ipsas ambiente.

Podoptères. Podoptera.

* Inter digitos inprimis expansa.

Dact yloptères. C heiroptères.

Dactyloptera. Cbeiroptera.

a. Membranâ natatui inserviente.

P.almé s. Palribata.

* Membraná inter digitos expansa.

Pa $1 \mathrm{~m}$ i p è des. palmipeda.

* Membranâ digitos involvente.

Pirnipèdes. Piuripedr.

B. Binis pinnis pedum anterionum analogis instructa.

A podes. Cetacés. Apoida. Cetaceg. 
A. TETRAPODES. QUADRUP㞷DES.

I. $\mathbb{F}$ i s s i p è d e s..

a. Onguiculés. Unguiculata. Dig 1 ta ta.

I.

\begin{tabular}{|c|c|c|c|c|c|}
\hline $\begin{array}{c}\text { I. } \\
\text { Bimana. } \\
\text { I. }\end{array}$ & $\underbrace{2 .}_{2 .}$ & $\begin{array}{l}\text { 3. } \\
\text { Pedimana. } \\
3 .\end{array}$ & $\begin{array}{c}4 . \\
\text { Palmigrada } \\
4 . \cdot\end{array}$ & $\begin{array}{c}5 . \\
\text { Plantigrada. } \\
5 .\end{array}$ & $\begin{array}{c}6 . \\
\text { Digitigrada. } \\
6 .\end{array}$ \\
\hline $\begin{array}{l}\text { lomme. } \\
\text { lomo. }\end{array}$ & 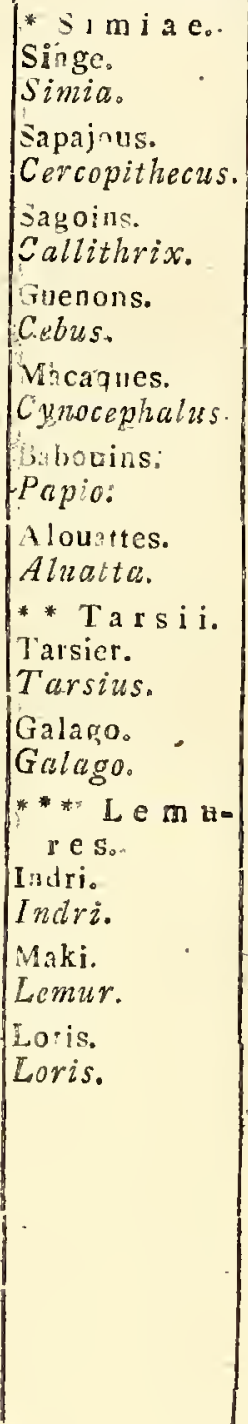 & $\begin{array}{l}\text { Parig:e. } \\
\text { Didlelphis: } \\
\text { rayure. } \\
\text { Dasyurus. } \\
\text { Phalanger. } \\
\text { Phalangevus. } \\
\text { * * } \\
\text { Wombat. } \\
\text { Wombatus: }\end{array}$ & 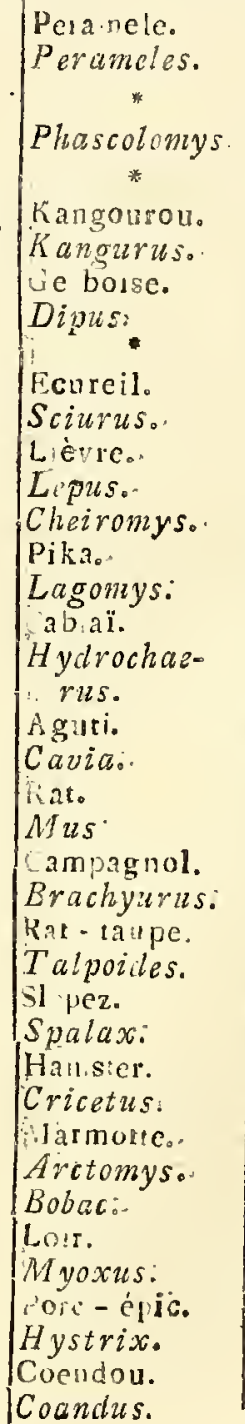 & 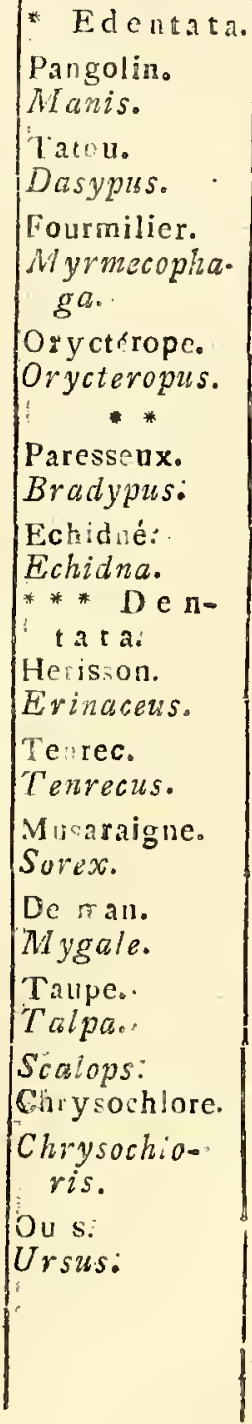 & $\begin{array}{l}\text { * Can in is } \\
\text { d uplicat is. } \\
\text { Blaireau. } \\
\text { Toxus. } \\
\text { 'oati. } \\
\text { Nasua. } \\
\text { Raton. } \\
\text { Lotor. } \\
\text { Kinkajou. } \\
\text { Kinkajus. } \\
\text { Marte. } \\
\text { Mustela. } \\
\text { Mouffète. } \\
\text { Mephitis. } \\
\text { Civette. } \\
\text { Viverra. } \\
\text { C a n in is } \\
\text { s i in } 1 \text { i c i- } \\
\text { bus. } \\
\text { Chat. } \\
\text { Felis. } \\
\text { Chien. } \\
\text { Canis. } \\
\text { Hyène. } \\
\text { Hyaena. }\end{array}$ \\
\hline
\end{tabular}


TETRAPODA. QUADRUPEDA.

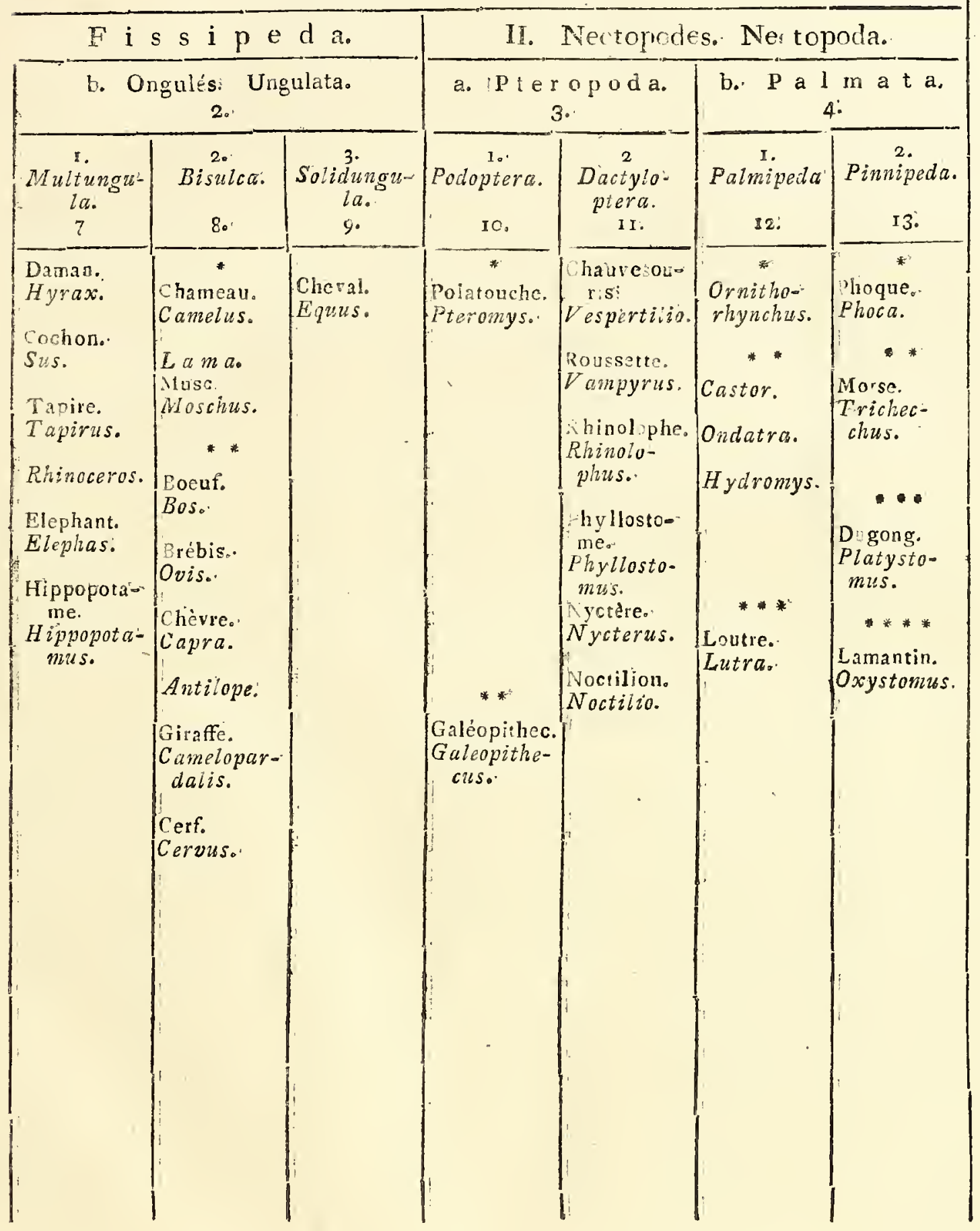


B. $\triangle P O D E S . A P O D A$

Cet a cés. Cerrecro.

* Edentata。

Baleine.

Balaena.

Baleinoptère.

Bulaenopters.

* Dentata.

Narhval.

Monodorz.

Catodons.

Anarnacus.

Physeter.

Pbysalius.

Delphinapterus.

Delpbiness.

Hyperoodon. 


\section{CLASSIS.}

\section{A V E S. O I S E A U X.}

I. Tibiis ad calcaneum usque plumosis.

Digitis membrana destitutis.

a. binis anticis totidemque pofticis. .... $\begin{aligned} & \mathrm{S} \text { c a } n \text { s o r e } 8 \\ & \text { Grimpèreaux. }\end{aligned}$

b. tribus anticis, vno vel nullo postico.

I. Vnguibus fortibus et aduncis. .. $\left\{\begin{array}{l}\text { A c c i p it res. } \\ \text { Rapaces. }\end{array}\right.$

2. Unguibus debilibus parum curvatis;

* digitis exterioribus liberis aut sola prima phalange coalitis. .... $\left\{\begin{array}{l}\mathrm{P} \text { a s s e r e s. } \\ \text { Passereaux. }\end{array}\right.$

* digitis exterioribus tota longitudine coalitis. ..... $\begin{aligned} & \mathrm{Pl} \text { a t y } \mathrm{p} \text { o d e s. } \\ & \text { Marcheurs. }\end{aligned}$ * * digitis anterioribus basi cinctis. $\left\{\begin{array}{l}\text { Ga } 11 \text { i n a e. } \\ \text { Gallinacés. }\end{array}\right.$

II. Tibiis parte inferiore pennis destitutis.

a. Digitis membrana auctis.

Tribus anticis vno vel nullo postico.

* Anticis membrana inter se connexis; postico soluto. ........ $\left\{\begin{array}{l}\text { A n s e res. } \\ \text { Palmipèdes. }\end{array}\right.$ * Omnibus membrana cinctis. . . $\left\{\begin{array}{l}\text { Plateretae. } \\ \text { Latirèmes. }\end{array}\right.$ ** basi membrana auctis. .. $\left\{\begin{array}{l}\text { Gra l I a e. } \\ \text { Echassiers. }\end{array}\right.$

b. Digitis membranadestitutis..$\left\{\begin{array}{l}\text { Cursores. } \\ \text { Coureurs. }\end{array}\right.$ 
II. O ISEAUX. I. LE DAS DE LA JAMBE GARNI DE RLJMES, TO!

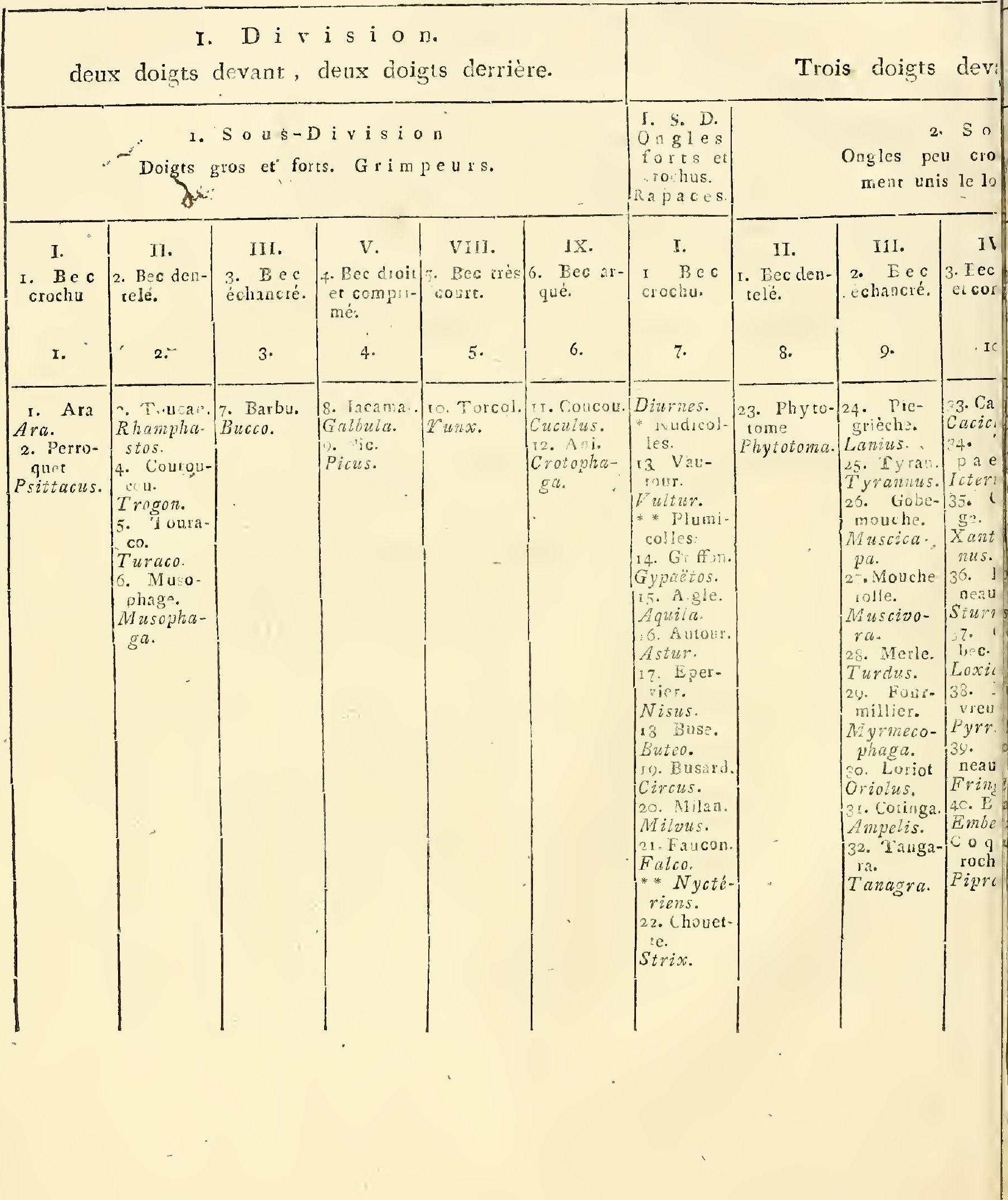




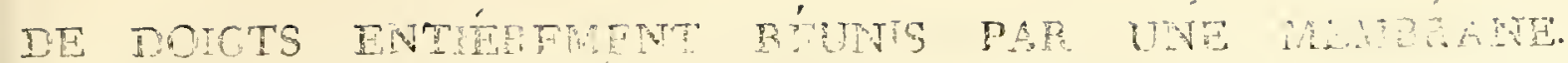

2. 1 i V i s i o il.

un doigt ou point de doict derrièe.

Division.

ts extérieurs libres ou seulela première phalange. Passereäux.
3. S o u s-D $\mathrm{i} v$ is i o r.

Digts extérteurs unis dalus presque route leur longueur. Platypodes.

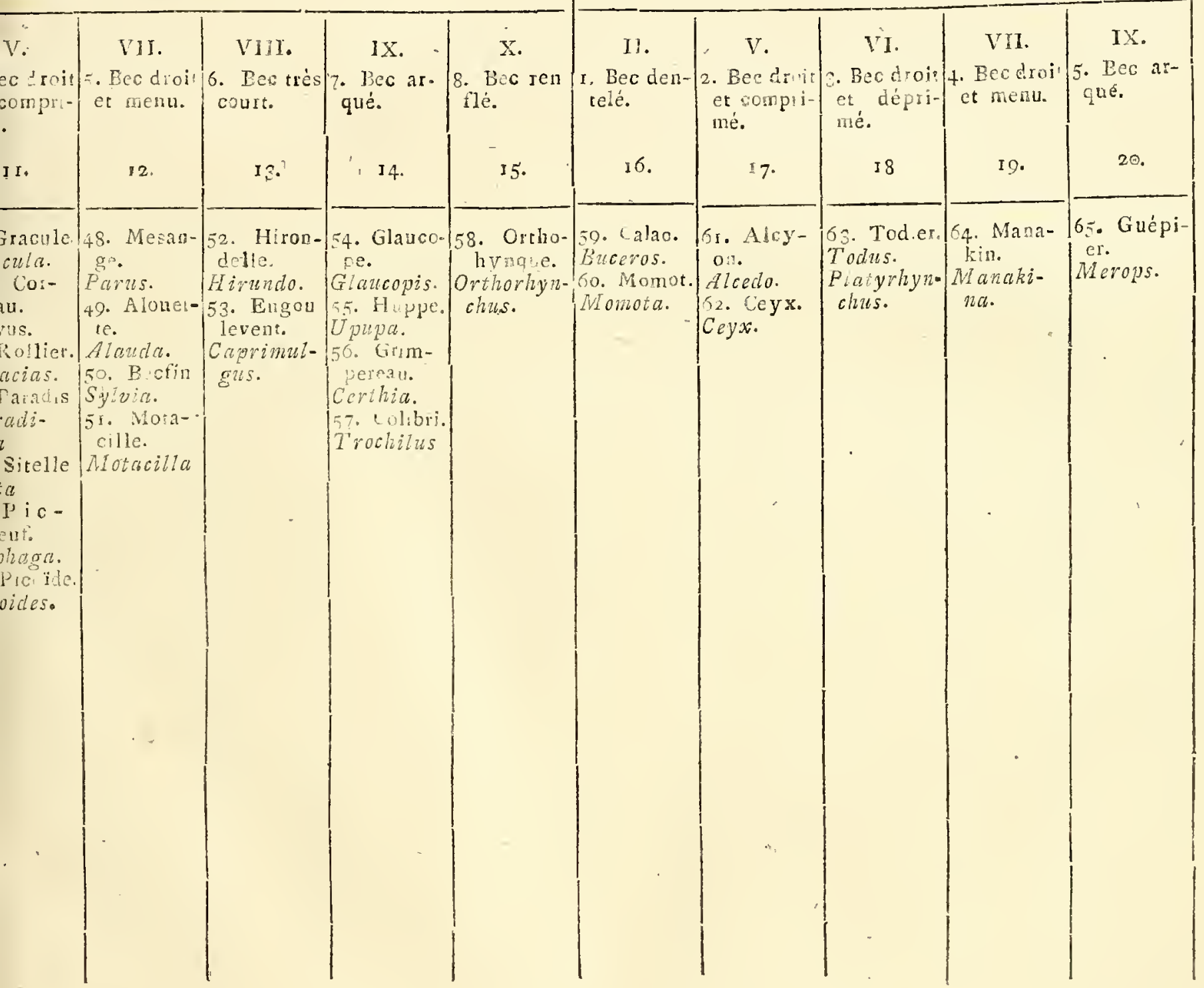




IT. OISEAUX IT. IE BAS DE LA JAMBE DENUÉ DE PLUM]

\section{Di vis i o n.}

Trois doigts devant, un doigt, ou pc

4. S D.

D. de devant ré nis à leur base par une membrane.

G a 11 in ac é s.

1. bec renflé.

2r.

66. Pigeon Columba.

67. Tetras Tetrao.

68. Pe : drix. Perdix.

6y Tinamoll.

Tinamus.

70 Tridactyle.

Tridacty-

lus.

7 i. Paon.

Pavo.

72. Faisan

Phasianus

73. Pinrade.

Numida.

74. Dindon.

Meleagris.

75. Hocco.

cirax.

i26. Outar-

ise.

Otis.

76. Penelo-

pe.

Penelope.

77 Gouan.

Guanus.
2. S. D i v is i o n.

4. Doigts réunis par une large membrane.

$L$ a $t i r$ è $m$ es.
Doigts de devant entièsement téunis par une membrane.

O iseaux d'cau. Palmipédes. b. bec cro- 2. b. den- 3. b. droit 4. b. droit 5. b. arqué 6. b. renflé chu.

22.

78. Flamand.

Phoenicopterus.

79. Alba-

irosse.

Diomedea. 80 Péléca-

noïde.

Pelecanö. des.

8i. Petrel.

Procella-

ria. 84 Petrel. ecoll.
25.

1:92. Sterne. 93. Avorette.
Recurvirostra.

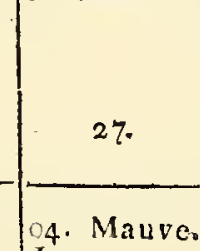

Larus. telé. et com- et menu. priné.

23. 24.

82. Canard. Anas.

83. Harle. Merous. 86. PlollPrion. Urinator.

87. Grèbe.

Colymbus.

88. Guil.emot.

Uria.

89. Alque

Alca.

go. Pingou-

in.

$P_{i}$ guinus

191. Man-

chot.

Aptenody-

tes
1. 11. I bec croco- 2. b. dentelé. et dépri

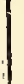

28.

95. FréFregatta. 96. Cosmoran. Carbo.
30.
97. Fou. Sula. can. 8. Phó- Pelecas ton. Phaeton. 99. Anhinga. Plotus. 


\section{PIUSIEURS DOIGTS RÉUNIS PAR UNE LARGE MEMBRANE.}

de doigts derrière.

3. So u s-D i v i s i o n.

Doigts réunis à leur base par une membrane.

Oisea ux de rivage. Echassiers.

\section{Division.}

2 3. ou 4. doigts très forts.

1. Sous-Division.

Doigts non réunis à leur base par une membrane.

Oiseaux coureurs.

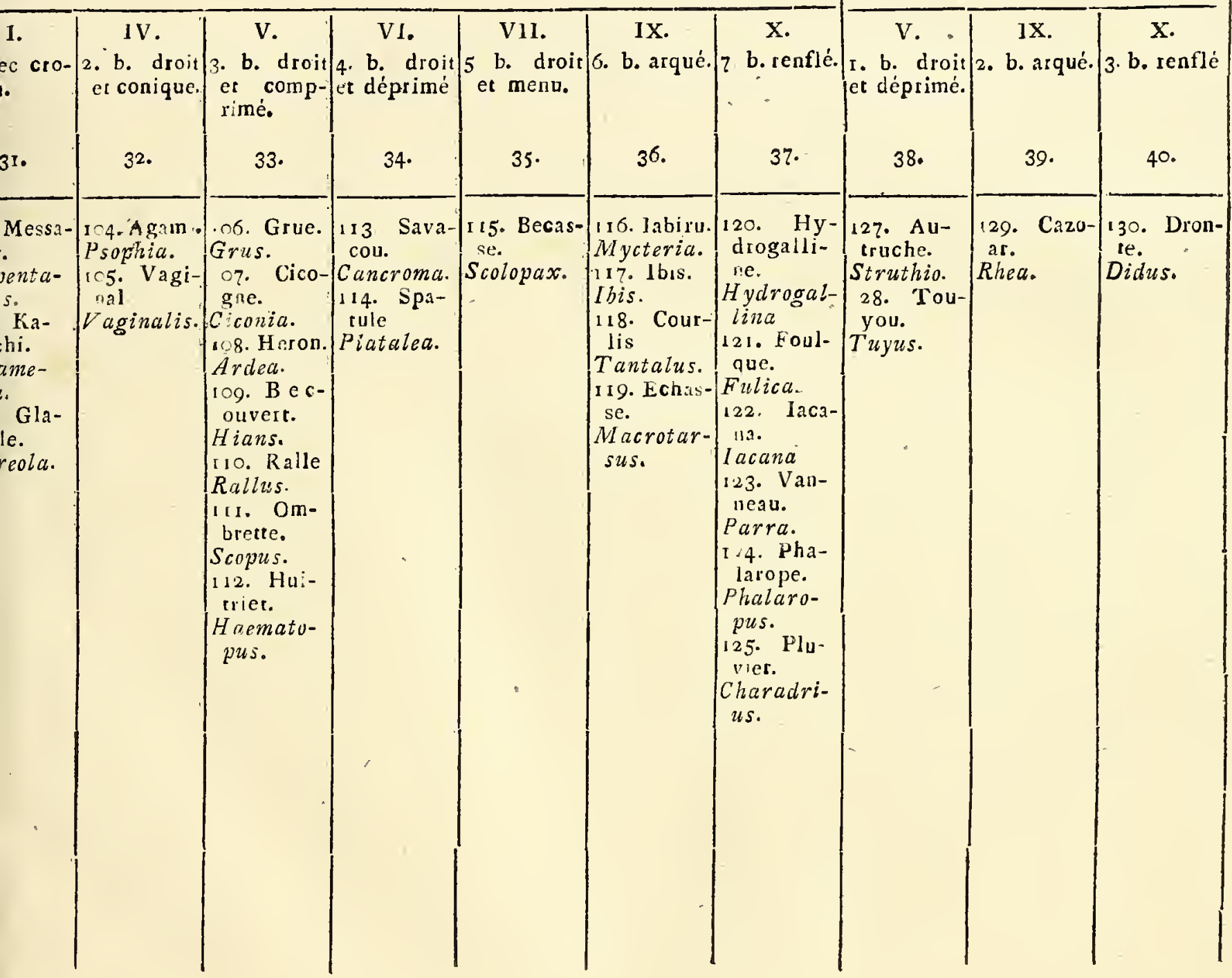


x

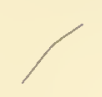

$+$ 


\section{REPTILES. REPTILIA.}

I. binis aut quatuor pedibus instructa. $\mathrm{P}$ ed a $\mathrm{t} a$.

I. digitis unguiculatis. Unguiculata.

* Corpus testis inclusum. Chelo n ia.

* Corpus fcutellis aut squamis tectum S a u ria.

2. digitis non unguiculatis. A chelat a. Batrachias II. Pedibus carentia, A poda. Ophidia. 
III. $\mathrm{R} E \mathrm{E} T \mathrm{~T} \mathrm{E} \mathrm{S}$.

Corps garni de deux ou de quatre pattes.

$$
P \text { e d a } t \text { a. }
$$

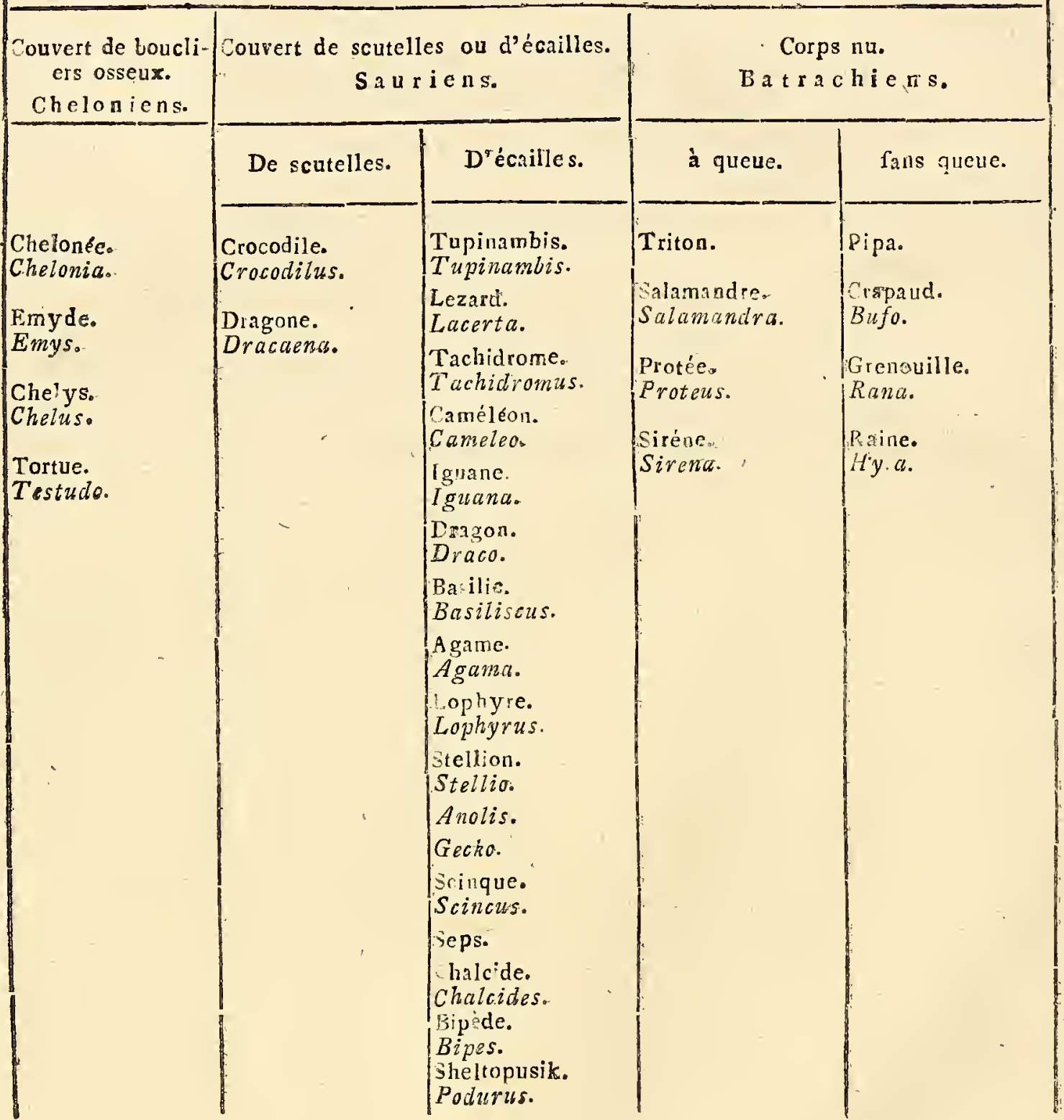


Corps alongés sans pattes. A pod e $s$.

O p h i d i e n s.

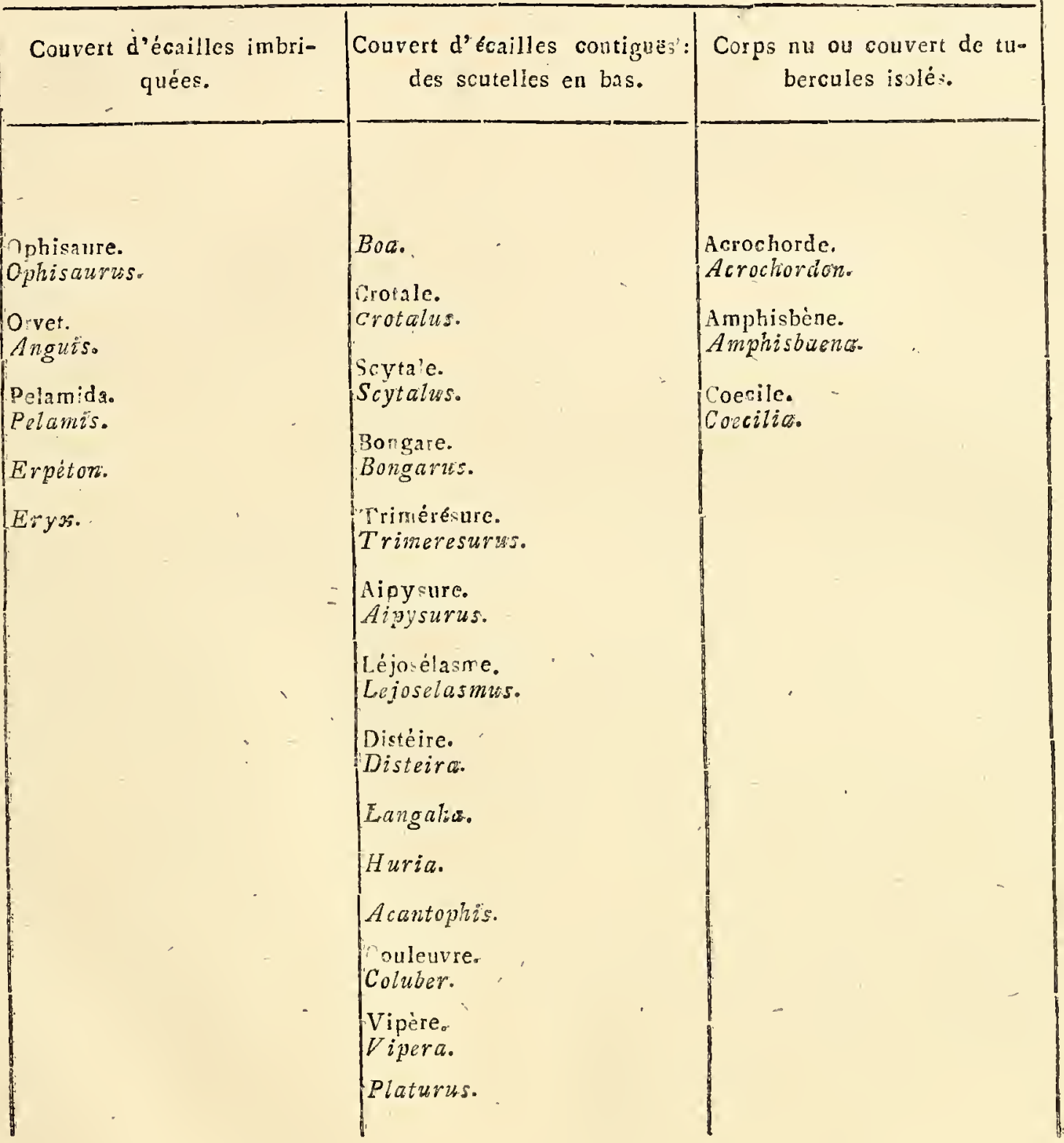




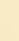


IV. Poissons. Pisces. L. Lacepède.

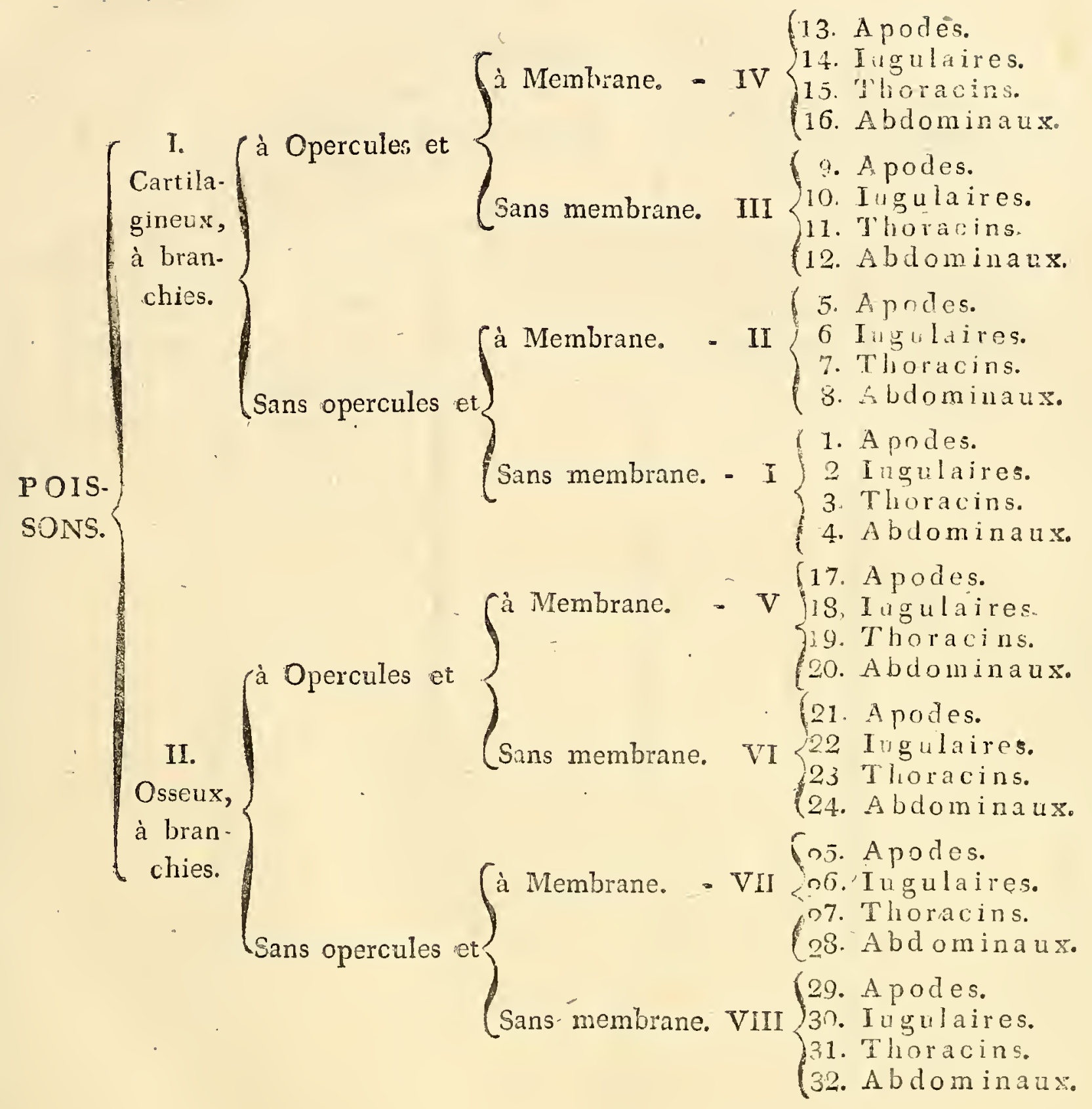




\section{Poissons I. cartilagineux.}

\section{L'épine dorsale composée de verièbres cartila.}

Point d'opercule branchial, ni de mumbrane branchiale. Chondropterygii.

1. Apodes.

Petromyzon L.

Gaítrobranchus.

Licep.

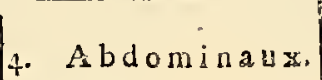
Raja. L.

Rhinolatus. Bl.

Squalus, $L$.

Prafts. Lath.

Audon L. C.
Point d'opercule branchial, une membrane brairchiale.

Branchiostegi.

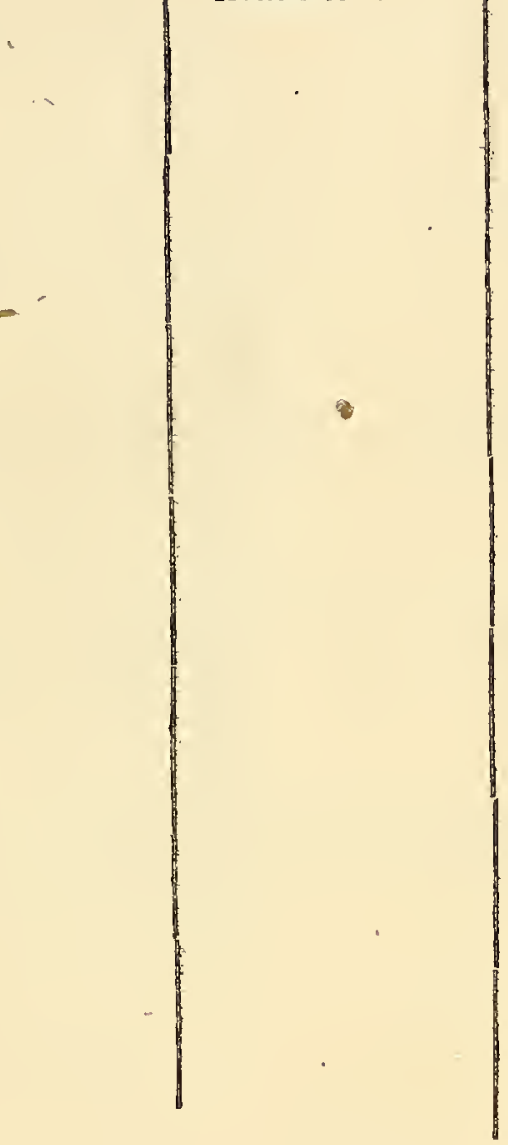

7. Thoracins. Lophius. L. Balleses, L.
3. Abdominaux Chimacra L.

Centriscus. L. Pegasus. L.

Macrorhynchus.

Lac. 
I. Pisces cartilaginei.

\section{gineuses. Foint d'écailles dans la plûpart.}

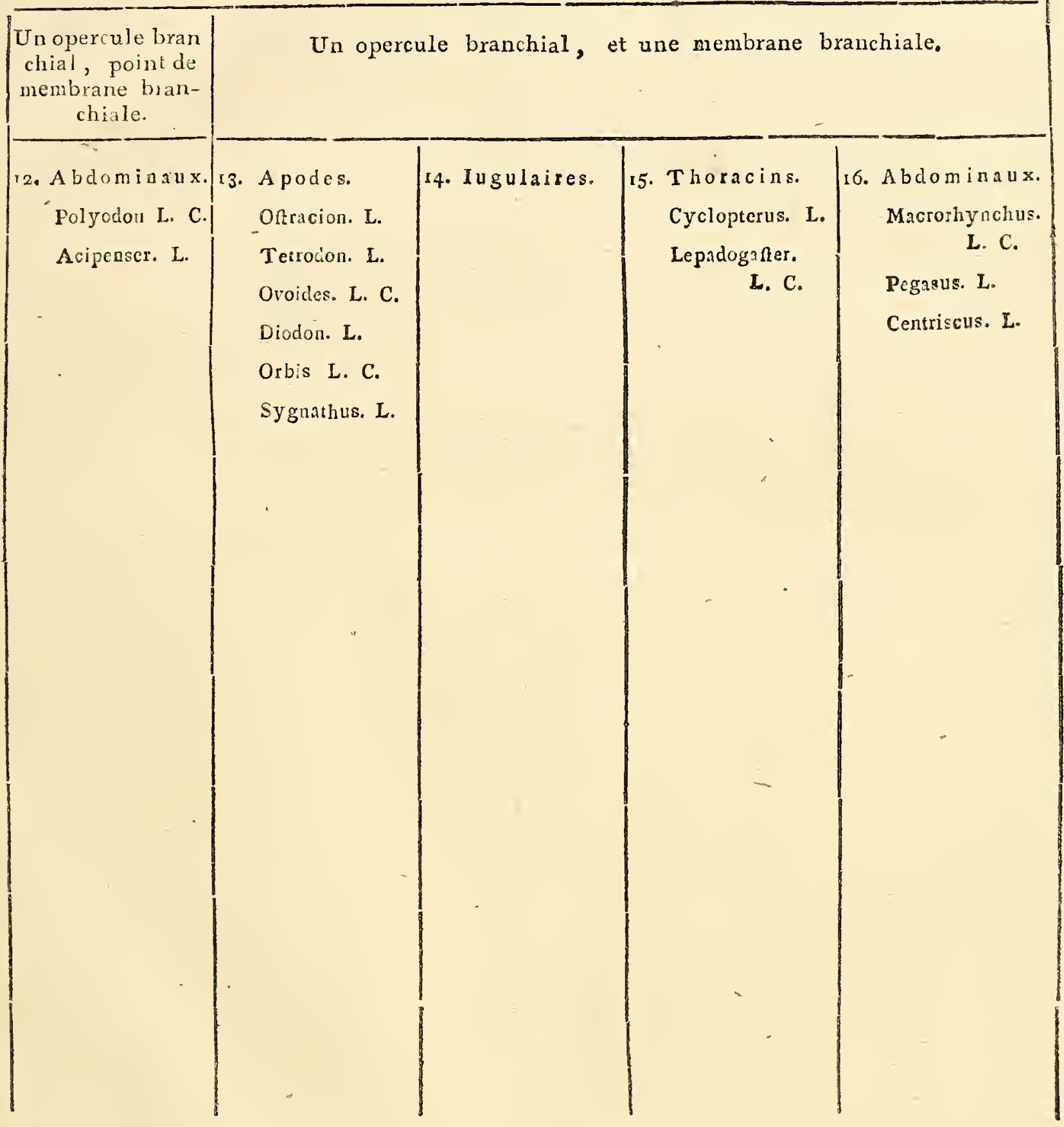


( 



\section{Poissons. II. Osseux.}

\section{L'épine dorsale composée de vertèbres}

Une ou deux nageoires fous la gox-Une ou deux nageoixes fituées à la par ge ou fituées en deçá des pecto-

r. Jugulaires. rales.

Kutrus. L.

Chrysontoma L. C.

Trachinus $\mathbf{L}$.

Gadus. L.

Batrachoides L. C.

Blennius. $\mathbf{Z}$.

Oligopodus, L. C.

Uranoscopus. L.

Callionymus. L.

Calliomorus. L. C.

Muraenoides. L. C.
Juguiares. tie inférieure du corps, au deffous dés nageoires pectorales.

2. Thoracici.

Thoracins.

Achirus. La Cep. pleuronectes. L. Capros. L. C.

Gallus. L. C.

Selene L C.

Argyrelosus. L. C.

Zeus. L.

Chaetodipterus. L. C. Pomadasys. L. C.

Enoplosus. L. C.

Acauthinion. L. C.

Chaetodon. L.

Poñacentrus. $L$. C.

Pomacanthus. L. C.

Eques. BI.

Glyphisodon. L. C. Acanthurus. L. C.

Apisurus: L. C.

A cantinepodus. L. C.

Perca L.

Holocentrus. Bl.

Micropteril. L. C.

Bodian. Bl.

Tacnianollis. L. C.

Lutjanus. Bi.

Centropomus. L. C

Dipterod :n. L. C.

Geforhyuchus. L. C.

Haspe. L. C.

Scarus. L

Sparus. L.

Nason. L. C.

Centrolophus. L. C.

Centronotus. L. C.

Caesiomo:us. L. C.

Cephalacanthus. L. C

Centropodus. L. C.

Scomber. L.

Scomberoides. L. C.

Scomberomorus. L. C.

Trachinotus. L. C.

Caranx. L. C.

Caranxomorus. I. C.

Caesio. L. C.

Leiagnathus. L. C.

Pogonias. L, C.
Plectorhyachus. L (C. Holog y mnosus. L. C. Ophicephalus. Bl. Cheilinus L. C. L t brus. L. C. Macropodus. L. C. Lonchurus. L. C. Trichopoda: L C. Monodactylus. L. C. Osphronenius. L. C. Kyphosus. L. C. Gomphosus. L C.

Leiostomus. L. C. Ponatomus. L. C. Cheilodipterus. Apogon. Mullus. L.

Trigla. L.

Prionotus. L. C.

Peristédion. -

Dactylopterus. -

Gyinucirus.

Ieriophorlls. $\longrightarrow$

Sco paena. I..

Macrourus. L. C.

Coryphaena. $L$.

Hcmipteronous L. C.

Coryphenodes. L. C.

Asp.dophorus.

Aspidophoroides.

Coris.

Cheilio. -

Gobiesox.

Gobius. L.

Gobioides. L. C.

Sobiomorus.

Gobiomoroides. -

Echeneis. $L$.

Bostrychus. L. C.

Bostrychoides. -

Cepola. L.

Taenioides. L. C.

Lepidopus. -

Hiátula. 
osseuses. Peau couverte d'écailles.

Une ou deux nagecires fituées fous le ventre, derrière les pectorales.

3. Abdominaux. Abdominales.

Cirnlitus. L. C.

Cheilodact ylus. -

Dorsuarius. -

Xyster. -

Mene. -

Gastcropelecus. -

Salmo. L.

Osmerus. L, C.

Coregonus. -

Characinus -

Serrasalınus. -

Elops. -

Megalops. -

Mormyrus.

Cyprinodon. -

Cyprinus. L.

Clupea. L.

Clupanodon. L. C.

Mystus. L. C.

Buro. -

Polynemus. B1.

Polydactylus. L. C.

Mugil. -

Mugiloides. L: C.

Chanos. L. C.

Mugilomorus. L. C.

Tripteronous L. C.

Anableps. -
Argentina. $L$

Hydrargira. L. C:

Stolephorus. -

Exococtus. L.

Pol ypterus Gff. H.

Sromberesox.

Sphyraera. -

Esox. L.

Syuodus. L. C.

Lepisosteus. -

Notacanthus. -

Loricaria. L.

Hy postomus. L. C. Corydoras. Gff. H.

Ageneiosus. L. C.

Macroramphosus. -

Centranodon. -

Plotosls. -

Tachysurus. -

Cataphrastus. L.

Pogonathus. L. C.

Doras. :-

Amia. -

Misgurnus. -

Cobitis. L.

Fundulus. L. C.

Colubrina. -

Butyrinus. -

Aulostomus. -

Solenostomus. -

Fistularia. L.
Point de nageoire ventrale. 4. Apodes. Apodes.

Rhombus. L. C.

Stromateus. L.

Sternoptyx. L. C.

Comephorus, -

Anarhichas. $I$.

Makaira. L. C.

Xiphias. L.

Macrognathus. L. C.

Ophidium. -

Odontognathus. -

Ammodytes. L.

Regalecus. L. C.

Trichiurus. L.

Triurus. L. C.

Leptocephalus. I. C.

Stylephorus. L. C.

Muraena. L.

Ophisurus. L. C.

Gymnotus. 'L.

Gy mnothorax. Bl.

Synbranchus. Bl.

Gymnomuracna. L. C.

Monopterus. -

Muratnoblenna. -

Sphagebranchus. -

Caecilia. - 

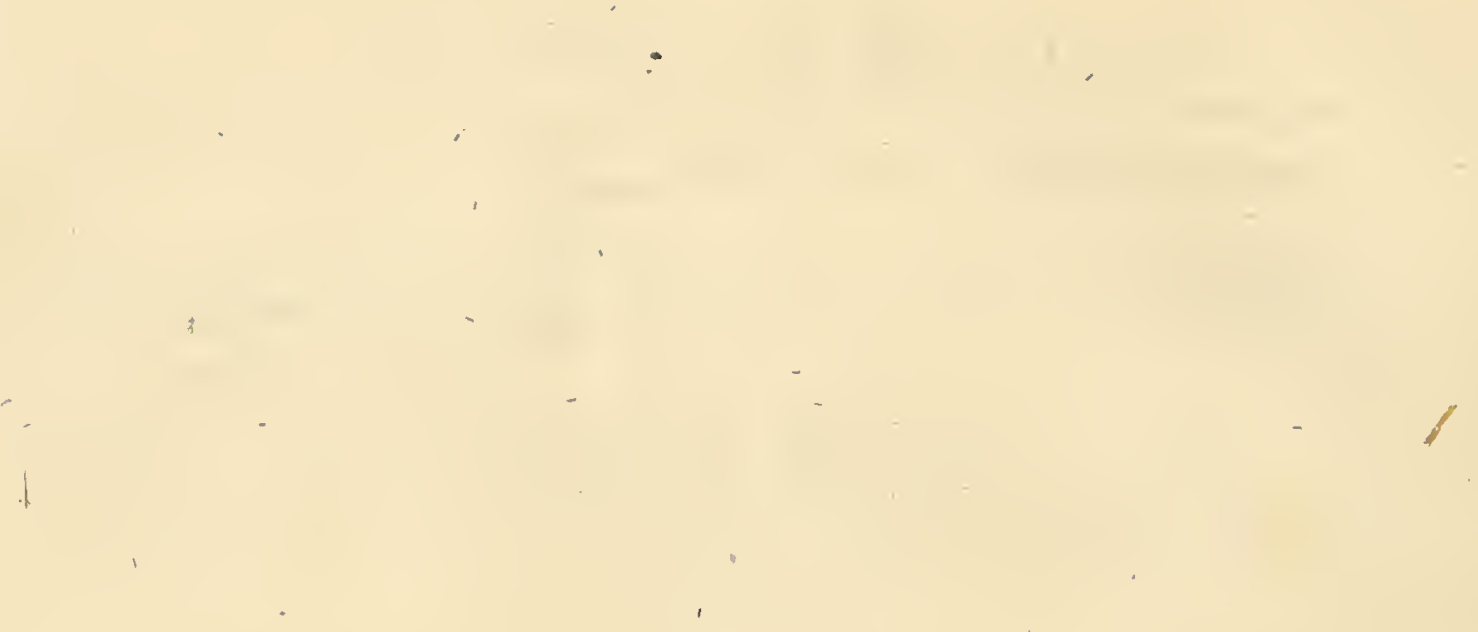

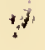

है 



\section{Mollusques. Mollusca Lam.}

I. Cephalés. Une tête diftincte et mobile; fouvent des yeux.

à Corps nu; point renfermé dans des coquilles. M. nus.

à Corps recouvert par une véritable coquille。 Moli. conchylifères.

* Animaux 1i. bres, nageant vaguement.

Sepia $\mathbf{L}$.

Loligo. Lm.

Octopus. Lm.

Lernaea. L.

Clio. L.

Pneumcderme.

Cuv.

Pterotrachea. Ik

Dosis. I.

Tritonia. Im.

Glaucus. Fft.

Eolides. Cuv.

* rampant

fur un ventre

large.

Laplysia. $\mathbf{L}$.

Limax. L.

Parmacella.Cuv.

Onchidium. Bch.

Tethys. L.

Bullaea. Im。

Sigaretus. Ad.

Phillidia. Cuv.

Scyllaea.

Pleurobranchus.

Cuvo

Coquille uni-Coquille univalve, uniloculaivalve recou. re, fpirivalve engainant lani vrant l'animal. Osabrion

Oscabrion. Br.

Patella L.

Fifiurella. $L \mathrm{~m}$.

Emarginula. I.m.

Concholepas.

Fv.

Crepidula. Lm.

Calyptraca. Lm.

.

Pur$$
\text { P }
$$

Buccinum. Lm.

Eburna. L.m.

Tercbra. Lm.

Dolium. -

Harpa. -

Cassis. -

Pterocera. -

Rofteliaria. -

Mures. L.

Fusus. Lm.

Pyrula. -

Fasciolaria. -

Turbinellus. -

Pleurotoma.

Clavatula. mal.

Cerithium. -

Stomatia. -
Coq. univalve, nultiloculaire, renfermant l'animal.

Nautilıs. L.

Ammonites. -

Planulites. -

Nummilites. Br.

Spirula. Im.

Turrilites. -

Baculites. -

Orthocera. -

Hippurites. -

Bclemnites. -

Discorbites. -

Rotalites. -

Lenticulina. -

Lituola. -

Spirolina. -

Miliola. -

Anotoma. Fsch.

Helix. L.

Helicina. Lm.

Nerita. L.

Natica. Lm.

Teftacella. -

Renulina. -

Gyrogona. -

Haliotis. -

Carinaria. -

Argonauta, - 


\section{Vermes et Teftacea Alior.}

II. Acéphalés. Point de tête diftincte; point d'yeux.

à $\operatorname{corps} \mathrm{nu}$.

M. acéph. $\mathrm{nus}$.

Ascidia. L. Salpa. Fk. Mammaria. [m. Fodia Bisc.

à Corps revêtu d'une enveloppe folide, crêtacée composée de deux pièces.

M. acéphalés conchylifères.

à coquilles équivalves; $\mid$ à coquilles inéquivalves; à charnière...

* sans dentsol**avec dents. $*$ * Sans dents. $\left.\right|^{* *}$ avec dents. Pinna. $L$. Mytilus. Lo Modiola. Lm.; Anodonta. Glycimeris. G.

I. à une dent. Unio. Lm. Craffatella. Paphia. Mya. 2. à deux dents. Lutraria. Lm. Erycina. Solen. L. Sanguinolaria. Lm.

Capsa. - T.

Venus. -

Venericardia.

Arca. $\mathbf{L}$. Lm.

Cucullaea. Lm Pectunculus. Nucula. -

Isocardia. Cardita. Cyclas. Donax. L. Hippopis. Lm. Tridacne. Trigonia. 3. à quatre dents. Meretrix. Lm. Buccardium. Lucina. Galathea. Oftrea. Lm.

Avicula. -

Pecten. L。

Lima. Im.

Pedum. -

Crania. Hyalaca. Lingula. r. à une dent. Radiolites, - Chama. L: Vulsella. Corbulz. Lm. Dicerates. 2. à deux dents. Spondylus. L. Plicatula. Im.

Pandorar Terebratula. Anomia. Calceolă. Placuna. 3. à plusieurs dents. Perna. L. Crenatula. Im. ( 



\section{Annélides. Annulata. Cur.}

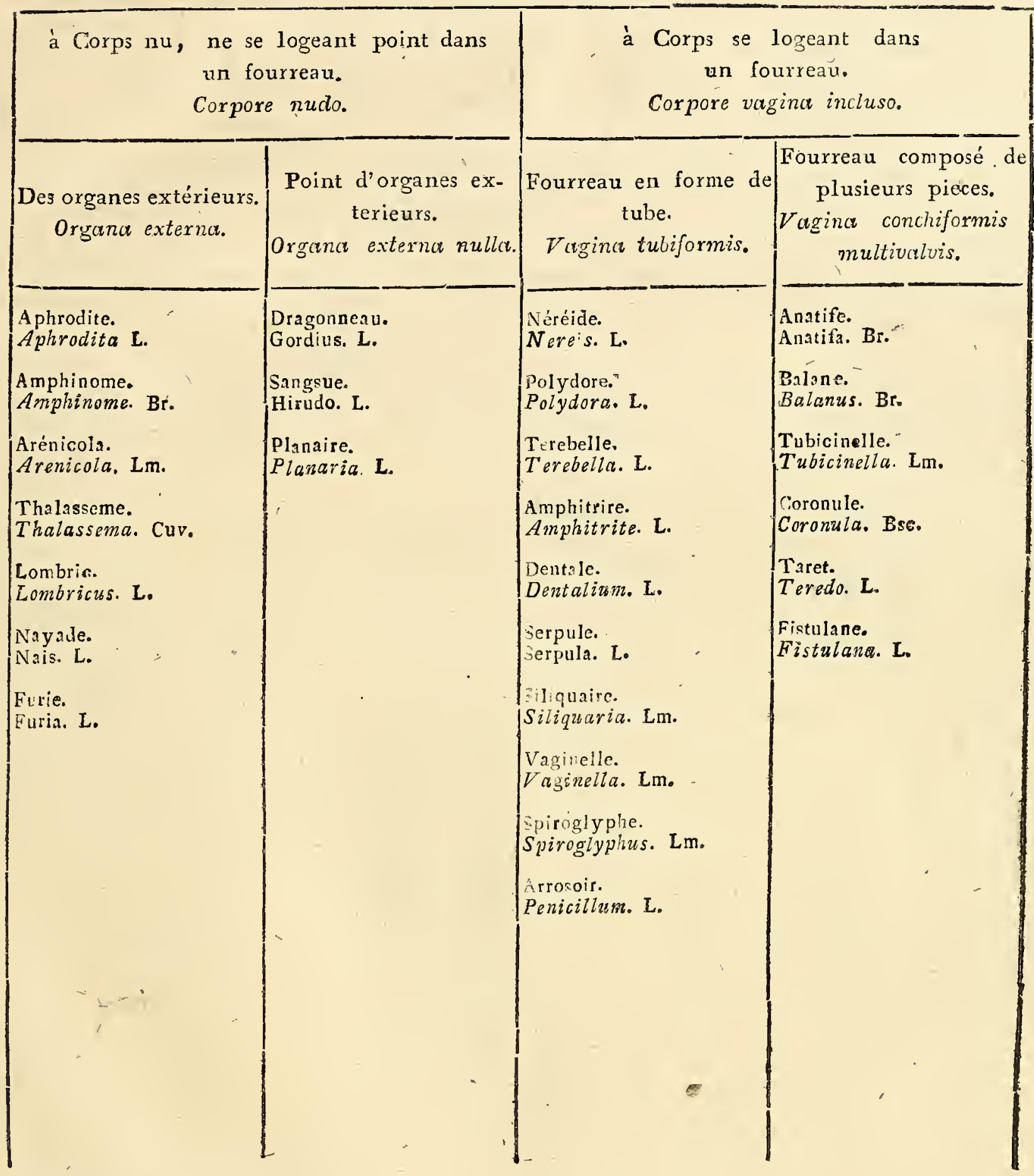


VII. Crustacés. Crustacea. Lam.

Corps couvert d'une peau membraneuse; cornée, ciypéacée ou bivalve. Yeux sessiles.

Corpore tegumento mmlaranceo, corneo, clypeato aut bivalvi; oculi sessiles.

Sessiliocles. Lam. Entomostraca. Latr.

Têt clypéacé ou bivalve.

Testa clypecitu inut bivolvis Operculésa. Thecata. Latr.

ב__

Têt couvrant tout le corps.

A s pidiota.

I. Xiphosura.

Limulus. $M$.

II. P n e u n 0 m u ra.

Caligus. M.

Binoculus. Gff.

1II. P h y 110 . poda.

Apus. Sch.
Têt bivalve.

Ostracoda.

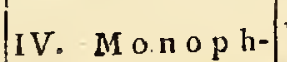
tha $1 \mathrm{~m}$ a.

Lynceus. $M$.

Daphnia: M.

Cypris $\mathrm{M}$.

Cythere. M.
Tèt nul.

Testa nullat.

$\mathrm{N}$ u s.

G ymota.

V. P s e u d o-

Cyclops. $\mathrm{Mr}$.

VI. Cephalota.

Polyphemus. $M$.

Zoe. Bosc.

Pranchiopoda.

Lm.
Corps couvert d'une croute cornée et calcaire; yeux pedonculés.

Corpus tegumento corneo-calcareo; oculi. pedunculati.

Pédiocles. Lam. Malacostraca. Latr.

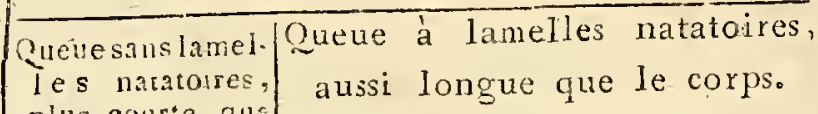
plus courte que
le corps. Cauda pinnis natatoris muilis, corpore brevior apicem, corpore ad minus longitudine.

Brachyuri Latr.

Kle y stag $n$ a. th a. F.

I. Cancerides. a. Platysmatii.

Remipes. Lit.

Hippa. T. Lm.

portunus. Dald. Pagurus. D.

Dromia. D.

Calappa. D.

Hepatus. Ltr.

Cailcer. L.

b. Vigiles.

Ocy pode. D.

Grapsu*. Lm.

Plagus a. Ltr.

IV. Palinuri.

Scyllarus. p.

Palinarus. D.

Forcclinna. $\mathbf{L}_{\mathbf{m}}$

Galathea. F.

V. Astacini.

Astacus. Gr.

Thalassina. Ler.

Alpheus. F.

Pauaeus. D:

11. Ox y h y nc hi.

Leucosia. $T$.

Maja. Lm.

Macropus, Ltr.

Lithodes. Ltr.

Corystes. -

Mictyris. -

Dorippe. D.

Osthyia, D.

Matuta. D.

Raniua. D.
Palaemon. D.

Crangon. 5 .
VI. Squili :-

Squilla. R.

Mysis. Ltr.

VII. Gam marin a e.

Phronima. Ler.

Talitrus. Bsc.

Gammarus. F.

Caprella. Lm. 


\section{Arachnoïdes. Arachnoida. Lam. Mitosata et Unogata Fabr.}

I. Corps à sept articulations pediféres ou plus. - Corpore segmentis pedigeris septem aut amplius.

IT. Corpj à trois sections pedifères. Corpore segmentis pedigeris tribus.

Tète distincte; des antennes:

Tête con'ondue avéc le corcelet. Point d'antennes. Coput a thorace discretum; antennae. Cuput cum thorcte unitum; antennae $\overline{\text { Quatorze paties, Plus que I } 4 \text { pattes, }}$ quatre antennes. deux antennes. I. Tetracera. 2. Myri a poda. $\mathrm{L}$ a t $r$.

1. Aseliota. Asellus. Gff. Idotes. F. Sphacroma. Ltr. Cymothoa. F. Bopyrus Ltr. II. O n is cides. Ligia. F. Philoscia. Ltr. Onisrus. L. Porcetlio. Ler. Armadillo, Ltr. nullate.

Huit pattes.

3. Acera Lat'r. L. Chatrogatha. Glomeris. Ltr. Julıs. L. Polydesmuss. Ltr. Pollyxenus. Ler.

II. Sygnatha. Scutigera. Ltr. Scolopendra. L..

1. L is ne ides. Mygale. Walk. Atypus. Lir. Erodon. Ltr. Drassus. $W$. Segetria. Let. Dy-dera. Ltr. Clubiona. Ler. reyroneta. Ltr. Aranca. L.

Therdion. W. scytodes. Ltr. Tetragnatha. Ltr. ippeirs. IV.

Uloborus. Ltr.

Tliomiscus. IV. Miciommisa. Lis. Dolomedes. Lir, Lycosa. I,tr. Eij:us. Ltr. Golticus. -

I1. Ped i pa 1 pi.

Phy ynus. Ol.

Theiyphonus. Ler.

III. scorpiunides. Scorpio. L.

Clatiter. Gft.

1 V. Pltalangita.

Galeodes. Ol.

Plislangium, L.

Trogulus. Ltr.

Sirn. Lir.

V. Pycnogonides.

IVymphor F.

Phoxichifus. Ler. Fycuogoumm. Bro
Vi. Acaricti ae. Trombidium. T. Erythacus. Ltr. Gornasus. Oribata. Acaus. L. VII. R i c i n i a sarcoptes. Ltr. Cheyletus. snatis. Bdella. Argas. Ixodes.Uropoda. VII1. H y d a chEylais. Ltr. Hydrachna. M. Limno:hares. XI. MicrophCaris. Ler. Leptus. Astoma. -
40.. A p terodic e ra. Ltr.

I. Thysa oura. 1. L e pis menae. Lepisna. L. Machilis. Ltr. 2. Podu re liac. Pudura. L.

Smynthurus. Ltr. II. P a rasit $i$. Ricinus. Geer. Pediculus. L. 


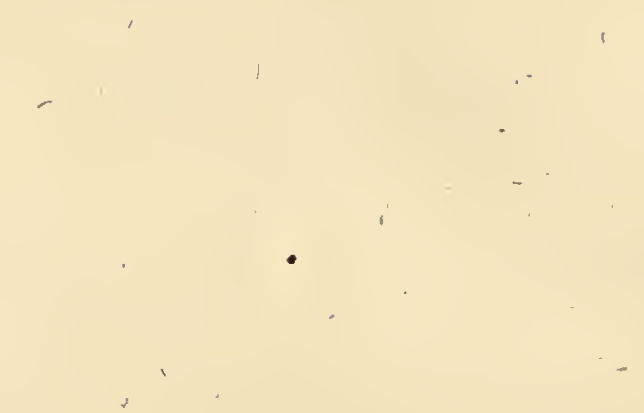




\section{I in $f$ e c t e s.}

Infecta, LAM. Pterodicera LATR.

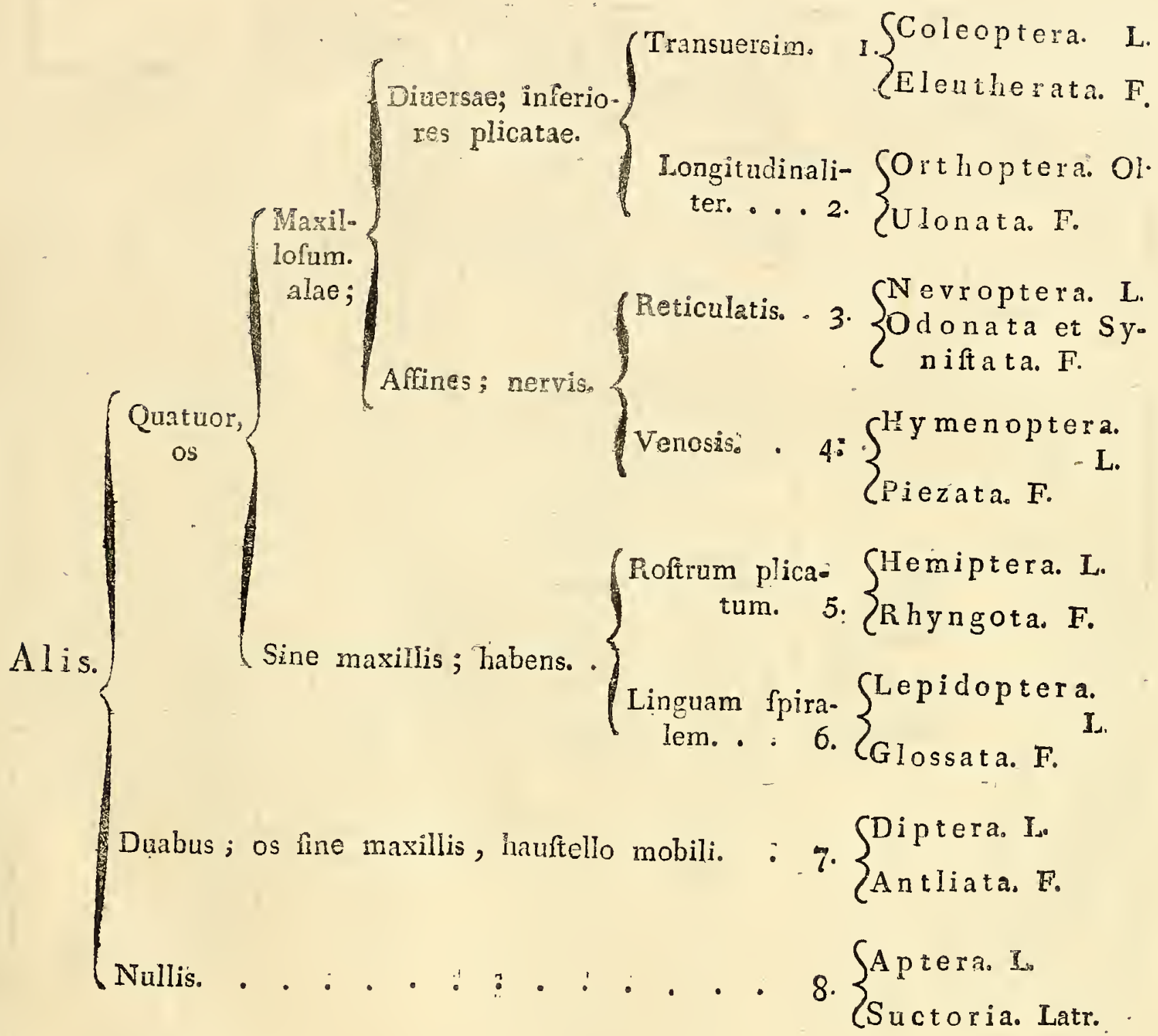




\section{I. Coléoptéres. Celeoptera L. Eleutherata F.}

\begin{tabular}{|c|c|c|c|c|c|}
\hline Tarses à & $\begin{array}{r}\text { à cinq artic } \\
P \mathrm{E} \cdot \mathrm{V}\end{array}$ & $\begin{array}{l}\text { les. Tarsis } \\
\text { T A IM E }\end{array}$ & $\begin{array}{l}\text { quinque arti } \\
\text { R E. S. }\end{array}$ & icubatiso & $\begin{array}{l}\text { Taxfes au nombre } \\
\text { pieds de devant; } \\
\text { derriere. Ta } x \text { s } \ddot{s} \text {. }\end{array}$ \\
\hline $\begin{array}{c}\text { à } 6 \text { pàlpes; } \\
\text { palpis fex; } \\
\text { ENTOMOPHAGL. }\end{array}$ & & $\begin{array}{l}\text { uatre palpes } \\
S A P R\end{array}$ & $\begin{array}{l}\text {, palpis qua } \\
\text { P I A G }\end{array}$ & & HETERO. \\
\hline $\begin{array}{l}\text { Anewhes fill- } \\
\text { fincs ou } \\
\text { fonformes. }\end{array}$ & $\begin{array}{l}\text { Autennes feti- } \\
\text { formes, filifor- } \\
\text { meroupectiné-s }\end{array}$ & $\begin{array}{c}\text { Aarenies plus } \\
\text { grosses a la } \\
\text { pointe. }\end{array}$ & $\begin{array}{l}\begin{array}{c}\text { Antennes en mas - } \\
\text { fue perfolice ou } \\
\text { folide. }\end{array} \\
\end{array}$ & 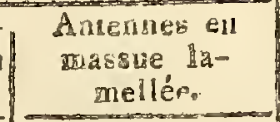 & I. $\mathrm{Nu}$ bili. \\
\hline 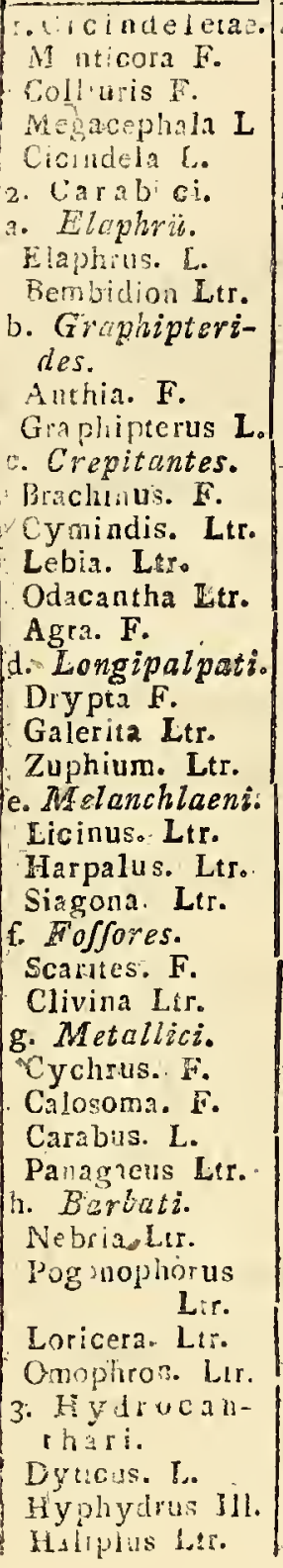 & \begin{tabular}{|c|} 
4. Sicruox I. \\
Bupreftis. L. \\
Melasis. F. \\
Elater. L. \\
Cebrio. F. \\
5. Malac \\
dermi. \\
Dascillus Ltr. \\
Elodes Iotr. \\
Cupes. F. \\
Drilus. Ol. \\
Lycus. F. \\
Omalisus \\
Geoff. \\
Lampyris. L. \\
Telephurus. \\
Sch. \\
Malthinus. \\
Ltr. \\
Melyris. F. \\
Zygia. F. \\
Dasytes. Pk. \\
Malachius. F. \\
Hylecoetus. \\
Ltr. \\
Lymexylon. \\
F. \\
Atractoceros. \\
P. B. \\
\end{tabular} & 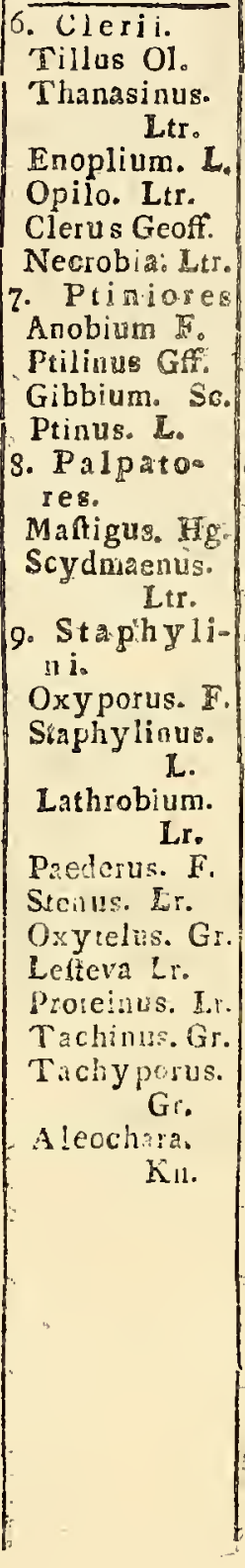 & 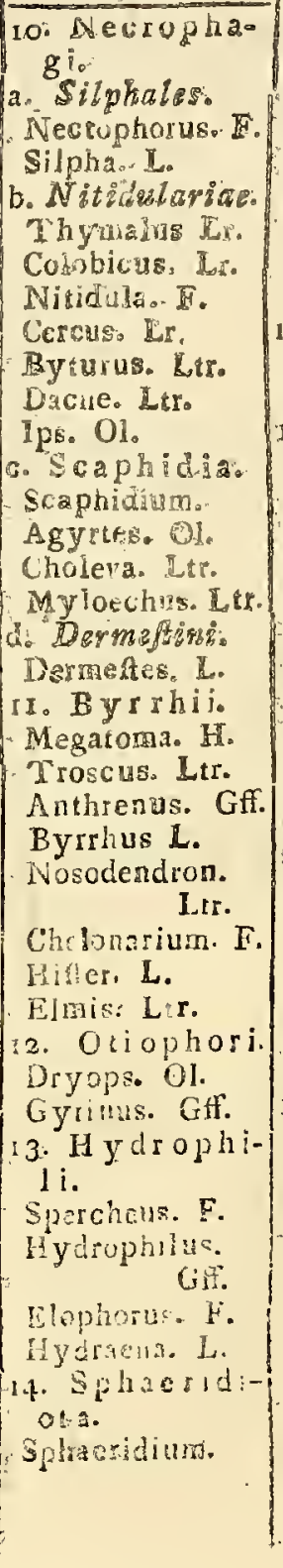 & 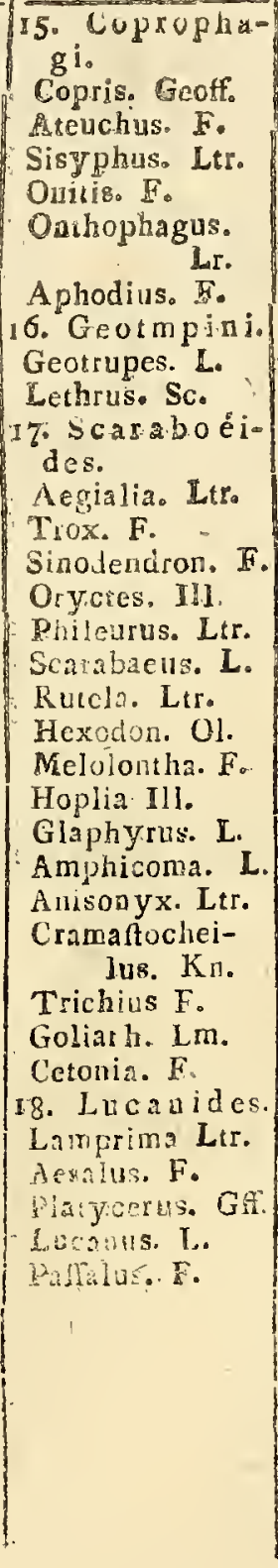 & 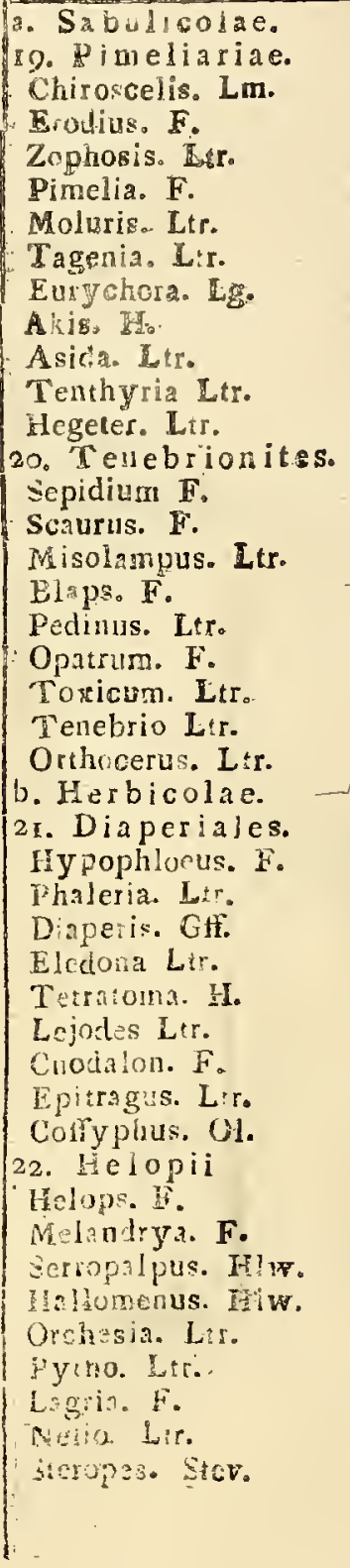 \\
\hline
\end{tabular}


Elytroptera. Vaginipennia. Alior.

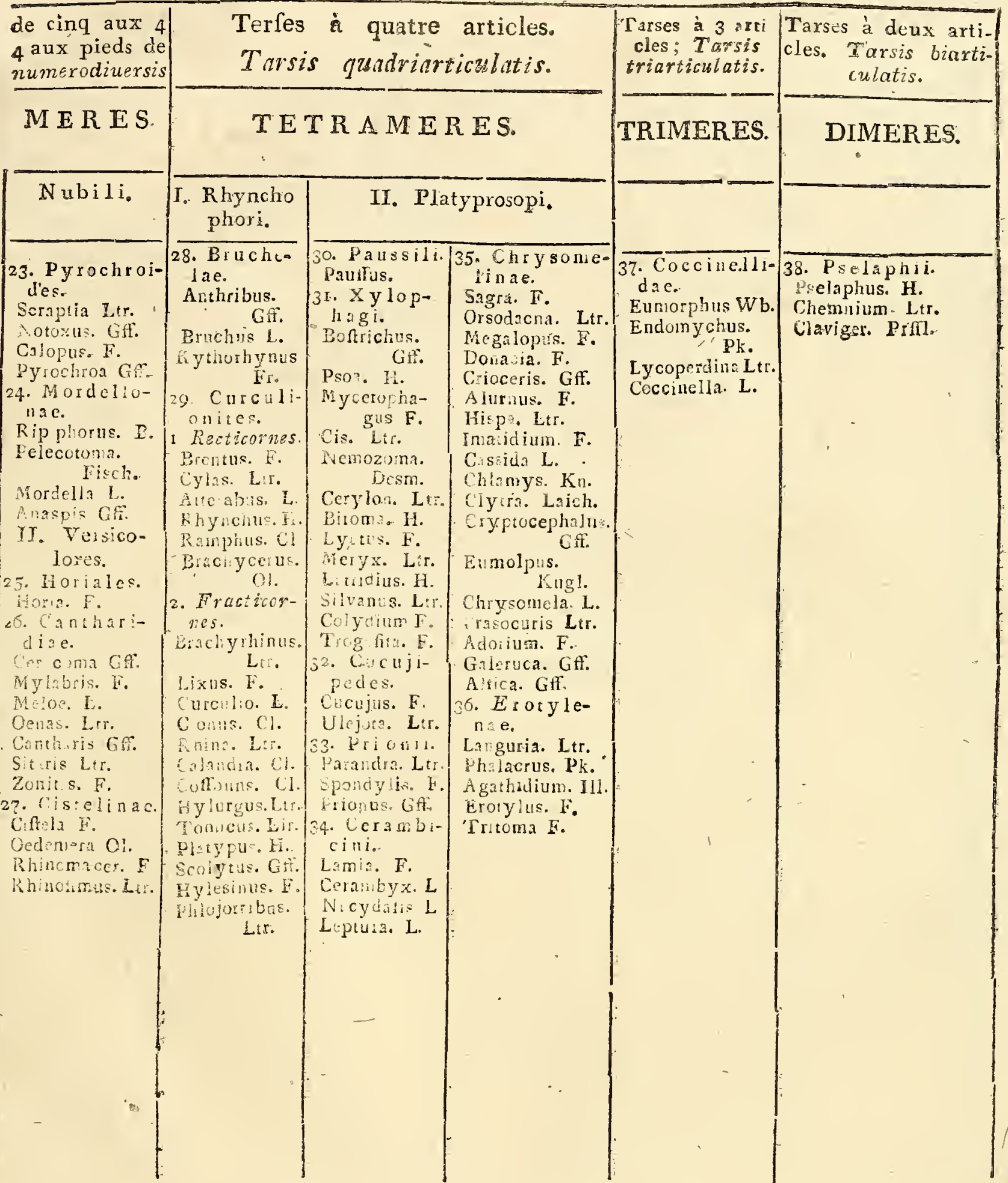




\section{2. Orthoptea; OLIVIER; Ulonata, FABRICI ; Orthoptères.}

\begin{tabular}{|c|c|c|c|c|c|}
\hline \multirow{3}{*}{$\begin{array}{c}\text { Elytres cou. } \\
\text { vrant deux } \\
\text { ailes plissées } \\
\text { en' partie en } \\
\text { éventail et } \\
\text { en partie } \\
\text { transver. } \\
\text { falement. }\end{array}$} & \multirow{3}{*}{$\begin{array}{c}\text { Elytres } \\
\text { couvrant } \\
\text { deux ailes } \\
\text { doublées fint } \\
\text { plement } \\
\text { dans leur } \\
\text { longueur. }\end{array}$} & \multicolumn{4}{|c|}{$\begin{array}{c}\text { Ely tres couvrant deux ailes plissées presqu'ento- } \\
\text { talité en éventail. }\end{array}$} \\
\hline & & $\begin{array}{c}\text { Tarses a } 5 \\
\text { articles; }\end{array}$ & $\begin{array}{c}\text { Tarses à } 4 \\
\text { articles; }\end{array}$ & Tarses & à 3 articles. \\
\hline & & $\begin{array}{l}\text { Antennes } \\
\text { fetacées ou } \\
\text { filiformes. }\end{array}$ & $\begin{array}{l}\text { A nutennes féta. } \\
\text { cées longues. }\end{array}$ & $\begin{array}{l}\text { Ant. fétacées } \\
\text { ou filiformes. }\end{array}$ & $\begin{array}{l}\text { Ant. filiformes ou } \\
\text { renflées au bout, } \\
\text { prismatiques, } \\
\text { ou comprimées. }\end{array}$ \\
\hline Eorficula. $L_{\text {s. }}$ & Blatta. L. & $\begin{array}{l}\text { I. Mantides. } \\
\text { I. Spectra. } \\
\text { Phylliumill. } \\
\text { Phasma Ler. } \\
\text { 2. Raptoriat. } \\
\text { Empusa IIl. } \\
\text { Mantis. L. } \\
\text { Maatispa Ill. }\end{array}$ & $\left\{\begin{array}{l}\text { 2. Losustaires. } \\
\text { Locuftariae. } \\
\text { Locufta. F. }\end{array}\right.$ & $\begin{array}{l}\text { 3. Grillones. } \\
\text { Grylliae. } \\
\text { Gryllotalpa.Ltr } \\
\text { Tridactylus. Ol. } \\
\text { Gryllus. L. }\end{array}$ & $\begin{array}{l}\text { 4. Acrydiens. } \\
\text { Acrydiang. } \\
\text { Tsuxalis. F. } \\
\text { Acrydium. F. } \\
\text { Tetrix. Lir. }\end{array}$ \\
\hline
\end{tabular}


IX. 3. Nevroptera L. Odonata er Syniftata. F. Nevroptères.

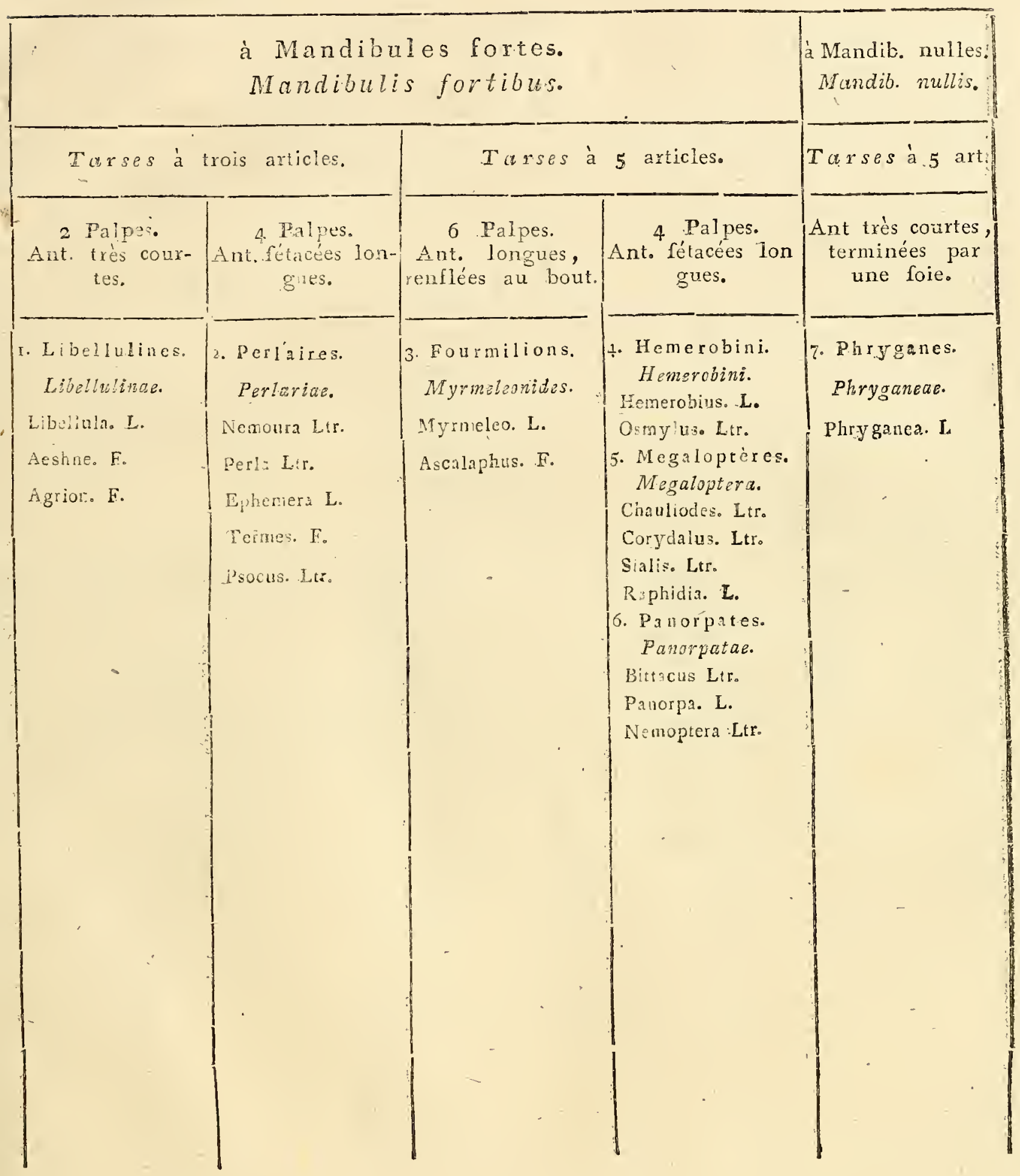




\section{4. Hymenoptera L. Piezata. F. \\ Hymenoptères.}

Femelles ayant à l'extrémité de l'abdomen une pièce en fcie ou en tarrière.

Porte - tarrière. Torebrantes.

Abdomen te / Abdomen tenant au coruant au cor-lcelet par un pédicule celet partou- ou par un anneau ré. (e) fon épais-

\begin{tabular}{|l|}
$\frac{\text { fcur. }}{\text { Sessili- }}$ \\
ventres. \\
\hline Phyti- \\
phaga. \\
\hline Tenthré- \\
dines. \\
Tethredi- \\
nes.
\end{tabular}

Cimbex. O]. Tentliredo. L.
Lophyrus.
Ltr. Hy lotoma. Ltr. Megalodonte. Nycteridium Fsch. Pamplicus. F.

Cephus. Ltr. Xiphydria. Ltr.

2. Uracerata. Urocerus. Oryflus. Ltr.

$\mathbf{P}$ u pophaga.

3. Diplole- 7.Chysides. pariac.

Ibalia. Ltr.

Figites. Chrysis L.

Parnopes. -

8. I c hneumonides. trupii.

Poctotrupes. Ichueumon. Lir. Helorus. - I. leptogaftri Diapria. ra.

2. roftrulati.

3. myftacini

4. longicolles. Leucopsis. F. 5. SphaeroceChalcis. F. phali. Cynips. $\mathbb{L}$. 6. truncati. 7. fasciati. 8. compressi. sa. Sigalphus. Bethylus. Lir. Ltr. Spacesion. - 9. EvaniaCleptes. - les.

Focilus. F.
Femelles et mulets ayant à l'extrémité de l'abdomeri un veritable aiguillon poignant.

Porte-aiguillons.

Aculeati.

Lángue membraneuse élar. Langue musculeuse longie, alongéé. gue étroite.

Nem oglossates.

Platiglossates. Diplolepis. -

Hedychrum. Sphex. L.

Ltr. 1. elongatae. 5. C'ynipse2. abbreviatae. Chlorion. Ler. Pelopocus. Pompilus. F.

Larra. F. Aliata. Ltr. 3. atomariae. Mellini-4. ambiguae. ores.

Psen. Ltr. 5. chelatae. Trypoxy-

10:1. -

Ceropales. - Cephaloie. Ltr Nyfron. -

17. Vespariae. I2. Crabroni- a. roftratae. tes.

$P_{E}$ mphredon. Eumeiles. a Ltr. b. murariae. Orybelus. - Odyuierus. Itr. 13. Bca bici-c. favosae. les. Stizus. Ltr. Vespá. L. Monedula. - Epipona Ltr. Bembex. F. Is. Philanto14. Scolie. i : $c$. 3) 1 y 9 a. Ltr. Scotia. F. res.

Philantus. F. Corceris. I. 16. Masarides Misaris F. 20. Alldienetae. Hylacus. F. Andiena. F. Dasypocia. Ltr. 
1X. 5. Hemiptera. L. Rhyngota F.

Hemiptères.

I. Bec pręnant origine à la partie antérieure et fupérieure de la tête.

Roftrum e copitis apice antico et fupero enascens.
II. Bec prenant origine à la partie inferieure de la téte.
I. Antennes plus longues que 2.- Antennes courtes, "cachées, la tête, placées au dessus placées au dessous des des yeux.

Antenice fupra oculos infertae, capite longiores.

I. Corisiac.

I. Scutellère. Scutellera. Im.

2 Pertatome. Pentatoma. 01.

3. Coré. Coreus. F.

4. Neide. Neides. Ltr.

5. Lygee. Lygaeus. F.

6. Capsc. Capsus. F.

7. Miris. Miris. F.

II. Cim icides. 3. reluvins.

8. Nabis. Ltr,

9. Reduvius. F.

Io. Zelus. F.

is. Plojere. Plojaria. Scop.

b. Ploteres.

12. Hydrometra. Ltr.

13. Velice Lito

I4. Geris. Lt..

c. Acantillas.

15. Punaise. Cimex. L.

16. Macrocephalus. Swed.

17. Phymata Ler.

18. Tingis. F.

19. Aradus. F. yeux.

Antennae fub oculis insertare cut absconditare.

II. Hydrocorisae.

20. Ochthére.

Ochtherus. Ltr.

21. Gaigulc.

Gaigulus, Ltr.

22. Beloftome.

Beloftoma. Ltr.

23. Náncore.

Naucoris. Gff.

24. Nèpe.

Nepa. L.

25. Rallatre.

Ranatra. F.

26. Notonectc.

Notonecta. L.

27. Corise.

Corisa. Gefo
3. Antennes très courtes. Antenncre brevissimae.

IV. Cica'dariae.

28. Cigale.

Cicada. L.

29. Cercopis. F.

3o. Ledra. F.

3r. Membracis. F。

32. Tettigonia. Ol.

33. Tetigometra. Ltr.

34. Fulgora. L.

35. Asiraca. Ltr.

36. Delphax. F.

V. Ps yllidae.

37. Psylla. Gft。

38. Livia. Ltr.

VI. Aplidii.

39. Thrips. L.

40. Puceron.

Aphis. L.

4r Alcyrodes. Ltr.

V. Gallinsecta.

42. Dorthesia. Bosc.

43. Coccus. L. 


\section{6. Lepidoptères. Lepidoptera L. Glossata F.}

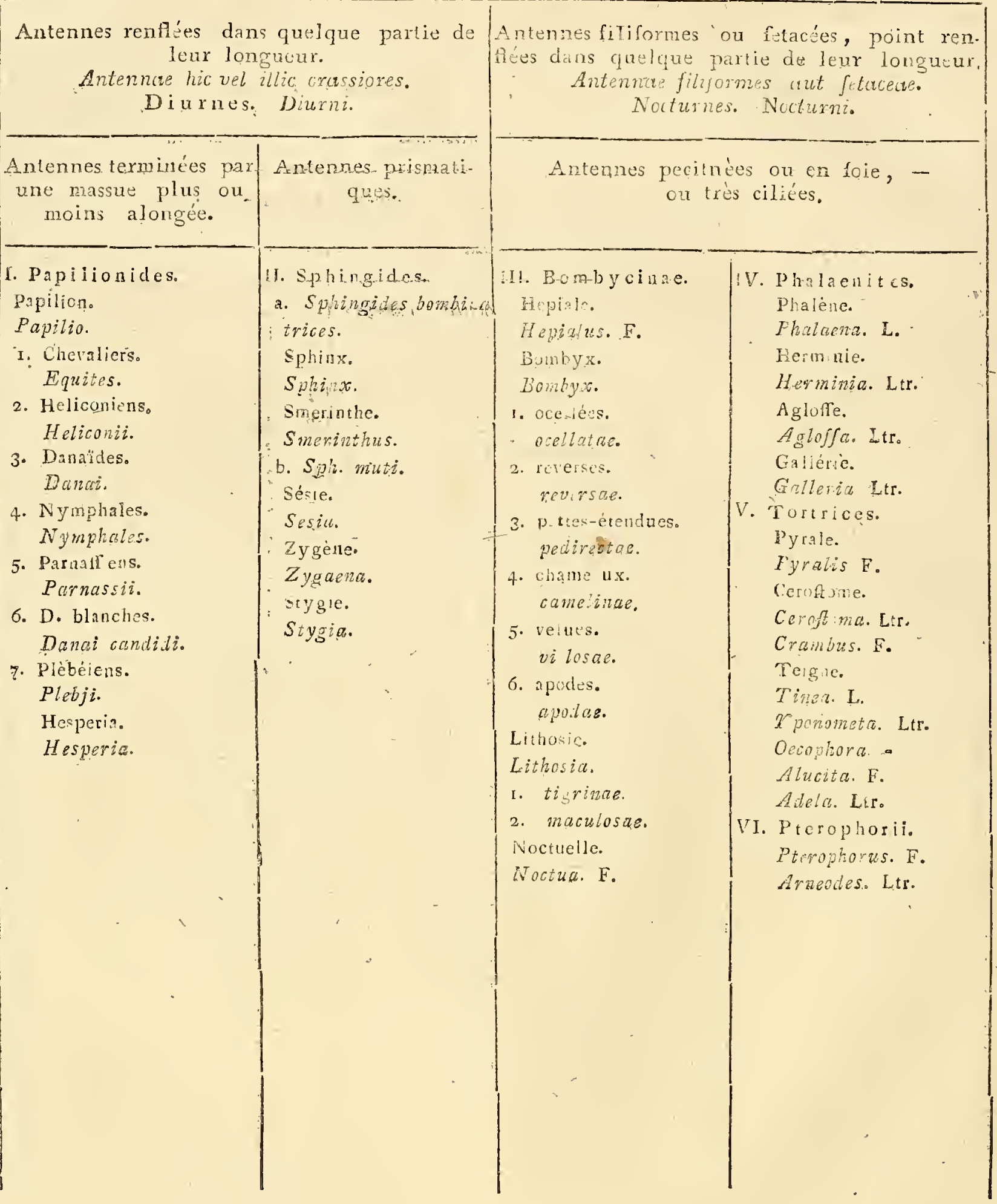




\section{7. Diptères. DIPTERA. L. Lm. Ltr. Antliata Fabr.}

Une trompe toujours faillante. Hauftello femper exserto.

Anrennes ayant au moins lept pièces diftinctes.

I. Tipulariae.

8. Colex L.

2. Cienophora Ltr.

3 Tanyptera Ltr. 22. U ia.

4. Tychoptera Ltr.

5. Limonia. Ler.

6. Molobrus. Lir.

7. Oligothrophu's. -

8. Corethra. -

9. Chironomus. -

го. Tanypus. -

11. Ceroplatus. -

12. Asindulam -

13. Rhyphus -

14. Mycetuphy -

15. Ceratopogon. -

16. recidon yia. -

17. Ps ychoda. -

18. Bibo. G.

19. S: mulium Lt:

20. Scathcpse. -
Antennes de deux ou 3 pièces, pas plus longue que la tête.
39. Rhynchoce-

47. Dol ichopus.
Trompe rétirée dans l'inaction ou nulle.

Hauftello retractili aut nullo.

Antennes 3 acticulées à pa. lette, ou à foie nue. \begin{tabular}{c|l} 
22. Uia. & VII. Siphuncu- \\
Ler. & Iara. \\
23. Ploas. & 37. Taigonia Ltr. \\
24. Cylle. & 8. Nemeftrinus.
\end{tabular}

III. A nphaius. Fich. tiract.

25. Muivo. 40. Taban us L. F.

26. Anthrax

iV. $A=i l i-$ ci.

27 Laphria Lir.

45. Therera. F.

43. Heptatoma. -

44. Cocnom yia. -

$L$ des.

29. Dasy-

pog n. Lis

3 . Dioct-

46. Rhagio. F.

Lst.

3r. Gony-

pes. -

V. E m pi-

des.

32. Plity-

pteta. Ittr.

33. Empis.

34. Sicus.

34. Sicus.
X. Strat-XII. Syrphiae. XIV.Coriaceae.

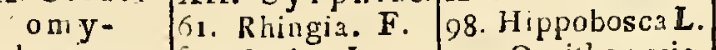

d a e. 62. Ceria. Ltr. 99. Ornithomyia.

48. Midas. 53. Psarus. Ltr.

F. 64. Aphritis. 100. Melopha49. Herme-165. Paragus. gus. tia. Ler. 65. Merodon. ror. Nycteribia. -

mys. F. 68. Syrphus.

5s. Odonto- 69. Eriltalis.

myia. Lir. zo. Volucella. F.

52. Beris. - XiII. Musci-

53. Ephip- des.

pium. - 71. Diopsis.

54. Sargus 72. Echinomyia.

55. Vappo.73. Ocyptera.Lir.

Ltr. 74. Phasia.

56. Nemo- 75. Musca. L.

telus. F.

XI. Cono-

ps a,riae.

57. Conops

77. Oscinis.

78. Camproriny

chus. Foch.

58. Zodion. 79. Kiftorhyn-

Ltr. chus. Fsch.

59. Myopa. 80. Ortites Lir.

F. 81. Dieiya. -

6o. Storno- 82. Tetancera. D. xis. Gfi:

83. Sepedon.

84. Luxocera Ltr

85. Calobata. -

86. Micropeza. -

87. Tephrites. -

88. Mosillus. -

89. L: Uxania. -

yo Lispe. -

91. Ochihera. -

92. Scenopinus. -

93. Piputiculus. -

94. Tynophora. -

95. Sphaerocera. -

95. Schizopa.

F sch.

o7. Phora. Ltr. 


\section{8. Aptères. APTERA. Lin. Suctoria. Latr.}

\section{Puce.}

Pulex. Lin. 
X. Vers. VERMES. V. Inteftins. Inteftinaux. Helminthes.

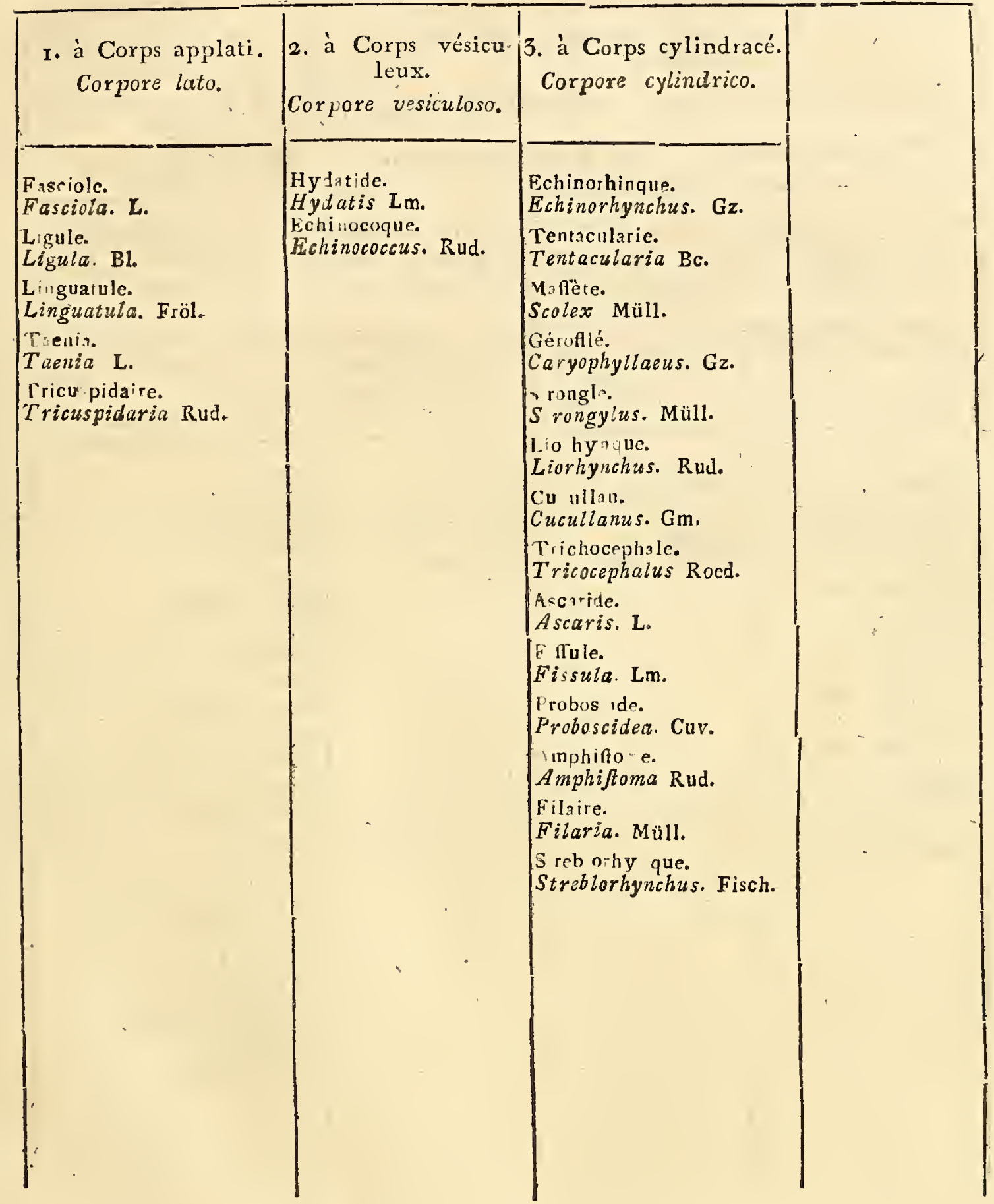




\section{Radiaires. RADIATA. Lam.}

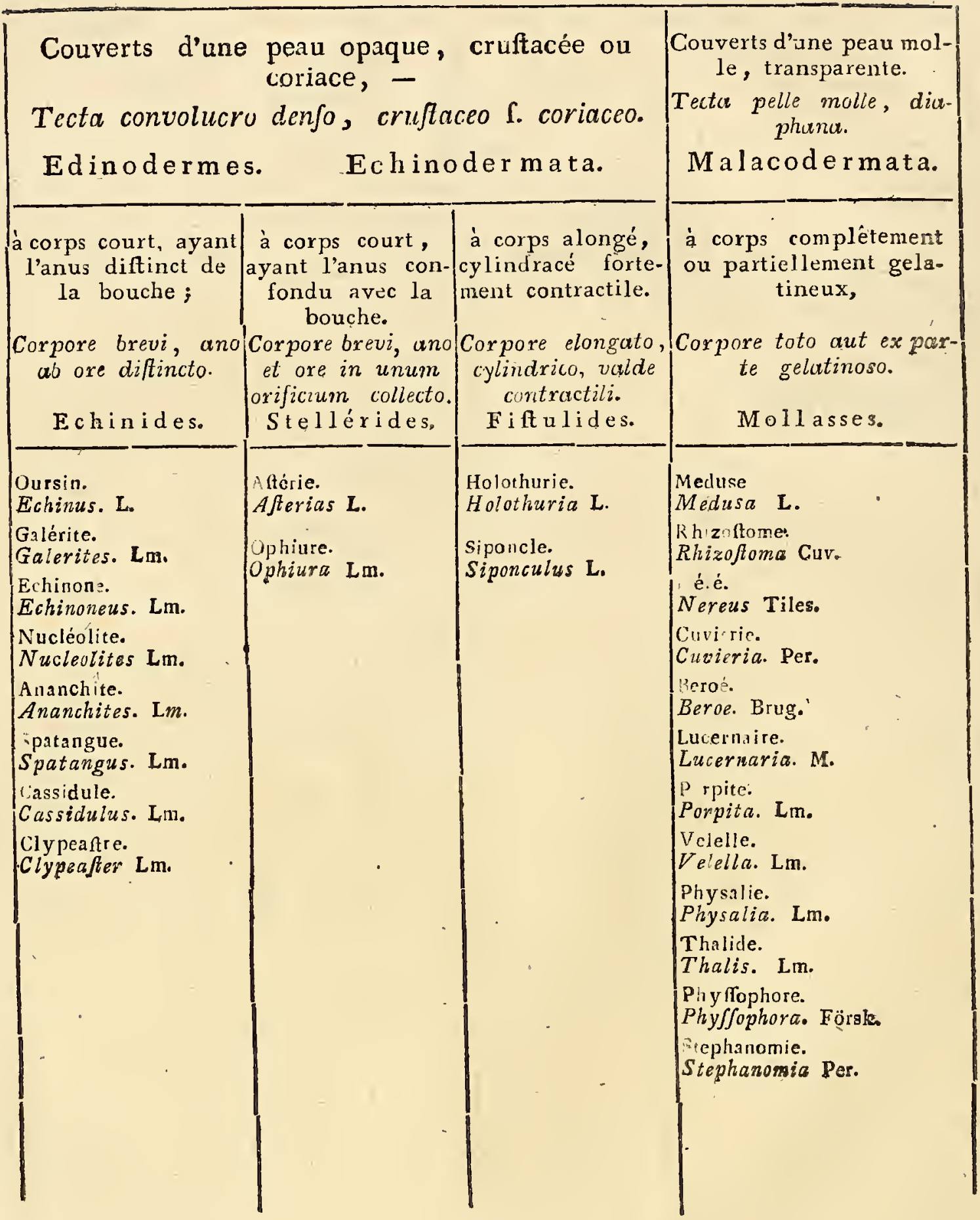


XII. Polypes. Polypi La marck.

I. Habentes circa os brachia radiata. $\left\{\begin{array}{l}\text { Polypes à rayons, } \\ \text { Polypi radiati }\end{array}\right.$ funt $\begin{cases}\text { vel nudi } & \left\{\begin{array}{l}\text { Polypi rad. nudi. } \\ \text { Polypes à rayons nus. }\end{array}\right. \\ \text { vel coralligeni }\left\{\begin{array}{l}\text { Polypi rad. coralligeni Lm. } \\ \text { faxigeni Dum. }\end{array}\right. & \left\{\begin{array}{l}\text { tectonourgi Lamethr. } \\ \text { lithophyti } \\ \text { Polypes cophyti }\end{array}\right\} \text { coliorum. }\end{cases}$

2. Habentes organa ciliata et rotatoria $\left\{\begin{array}{l}\text { Polypes rotifères } \\ \text { Polypi rotiferi. }\end{array}\right.$

3. Habentes nulla organa radiata fed $\{$ Polypes amorphes corpus amorphum 


\section{POLYPES. POLYPI LAMARCK.}

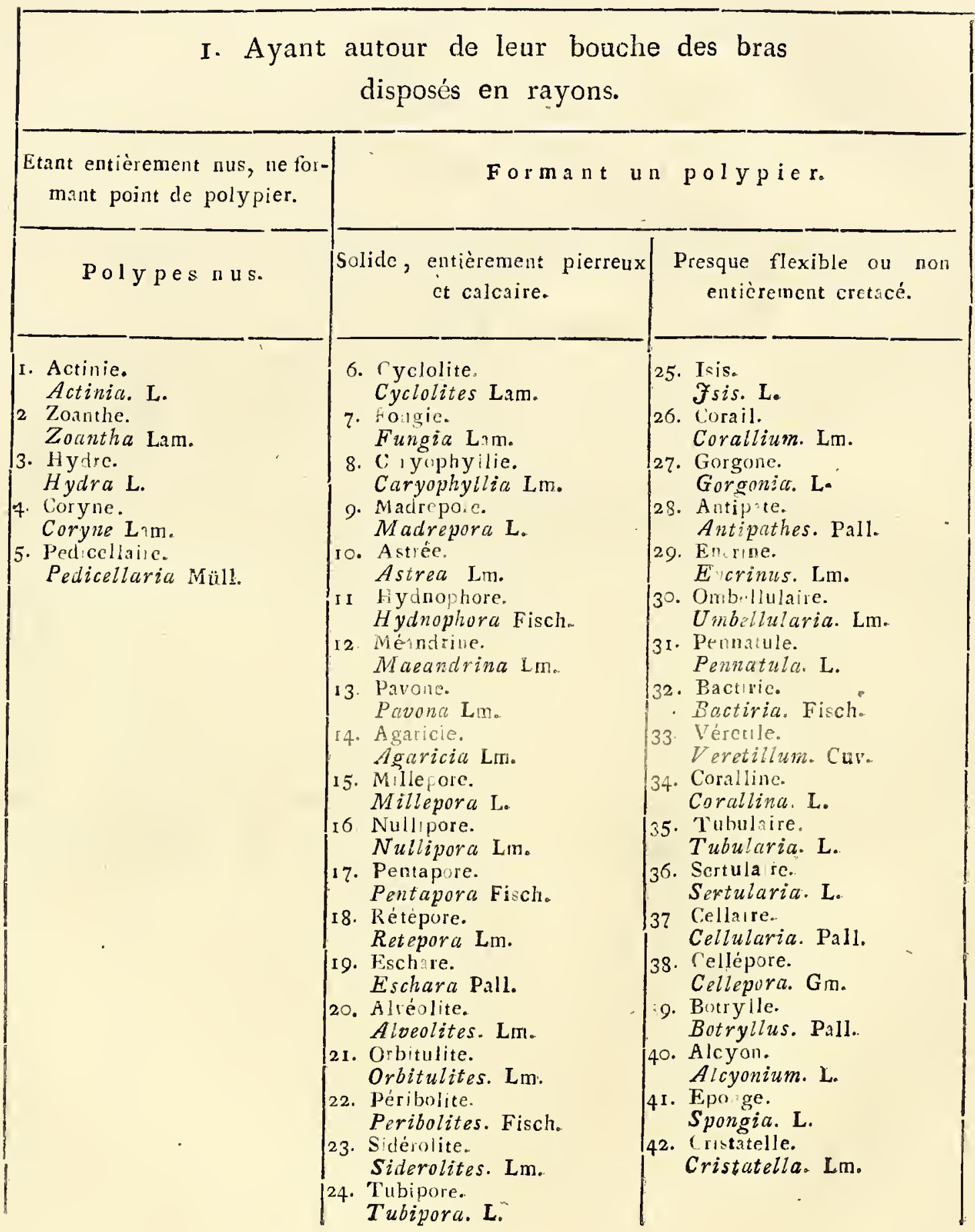


2. Ayant des organes ciliés et rotatoires.

Polypes rotifëres.

43. Vorticelle.

Vorticella. L.

44. Urcéolaire.

Urceolaria. Lm.

45. Brachion.

Brachionus. Müll.

3. Etant irréguliers sans bras rayonnans et sans organes rotatoires.

P o 1 y pes a morphes.

**Depourvus d'organes extérieurs. rieurs saillans.

46. Trichode.

Trichoda. M.

47. Trichocerque.

Trichocera. Cur.

48. Cercaire. Cercaria. $\mathrm{M}$.
49. Colpode.

Colpoda. M.

50. Vibrion.

Vibrio. M.

51. Protée.

Proteus. Brugu.

52. Volvoce.

Volvox. M.

53. Monade

Monas. M. 
TABLEAU SYNOPTIQUE

DES

M O L L U S Q UES. 


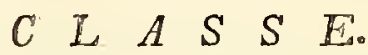 \\ DES MOLLUSQUES}

M. o I I u s c a.

\section{Mollusca. et Testacea Linnaei.}

Les. mollusques: ne forment qu'une très, petite: partie de la sixième et dernière Classe d'animaux de LiNNé, qui par un examen trop fuperficiel a réuni tous les: animaux fans: pattes articulées dans une feule et mème Classe Il a rassemblé fous le nom d'Intestins, Mollusques, Testacés et Zoophytes des animaux: dont l'organisation eft très différente; dont quelquesuns, furpassent de beaucoup en organes, les. Insectes, nonobftant leur fix pattes, articulées. Il en a féparé par des fousordres, d'autres: quil méritent d'appartenir à un ensemble naturel. Le c 'ubre PaLLAS est le premier qui, eximinant: la vraie nature de plusieurs de ces animaux fe fentit obligé de proposer un arrangement de ces êtres, par lequel il réunit les Limaces, et les Seches avec les univalves; les bivalves avee les Ascidies; et les ferpules comme troikieme ordre qui forme le passage aux Zoophytes ou. Polypiers, avec,les: Aphrodites $\left({ }^{*}\right)$. Ainsi. les obser.

(*) Cette observation intéressante fe trouve dans les Miscellaned p. 33 j je I"ajoute avec les mots de l'auteur pour ceux qui n"ont prs ce live a la main:, gqui a praejudicis ,hbero animo hune Tefaceorun. ordinem, quilis a LINNAEO et conchyliogis anie „cum omnibus tratitur', considaverite er non in testae calcareac accessoria nota con, fictas , fet animalia, quarum ha testae involucrum funt, perluftaverit, necessario s-perspiciet triplicem hic Mulluscorum gentem ab aucturibas invita natura corgergatum ,fuisse, quarum. fingula 3 gues fpecies tefta deftituas infer marina animalia adga cir. ,Sic euim unirersa Coctídum fou Testaceorum univa'vium fimilia Limacum Dori, dum, Tellidumque LINN. ideofle Lerneae, Argi EOHADS Hil renque Scyl-

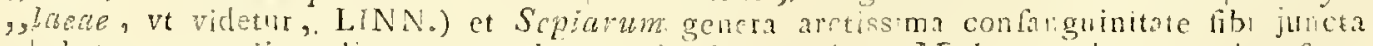

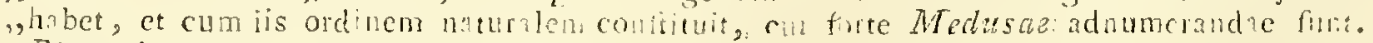

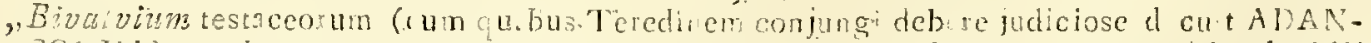

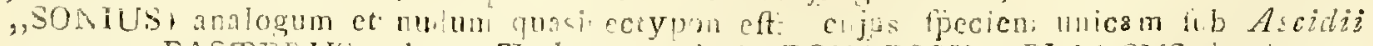
", nomme BASTERUS, plures Tethyomm tit!o BOHADSCH et PLAMCUS iccripce. ntibic ordini aninis valde cft et fulce adjungerdus multis videcur is, in qum Pri- 
vations de PALLAS prouvêrent déjä, il y a trente ans que la feparations des. Mollusques et des. 'Testacés formoient une division dans le fystéme qui $n^{2} e x i s t e$ point dans la nature. Les recherches de Fol, de Cuvifk, de LAMŕRKK, de Bosc ont éclairci cette partie à un point qu' on ne peut plus douter ni de'la place que ceite Classe doit occuper dans: l'échelle: aúmal, ni de l'arrangement des corps qui la composent:

Les MoIliusques, Mollizsca, nom quon a adopté pour cette Clase d'animaux fans vertébres, présentent: un corps charnu, mou, fans membres articulés, un coeuret un fystéme complet de vaisseaur ramenx, dans lesquels fe fait une vraie cilculation de leursfluides. Une partie deces vaissea ux forme un ou plasieurs reseaux, femblables aur. branchies des poissons, et qui comme dans ceuxci fervent à la respiration de ces animaux. Ils out une masse diftincté en guise de cérveau, de laquelle partent une moelle allongée et des nerfs.

Ces animaux font donc: des mieux organisés à tous égard’s parmi les animaux fans vertebres et approchent le plus des poissons: La Classe des mollusques doit donc fuivre celle des poissons et êtró par' consequent la 'premiere fur l'érlelle des animaux invertébrés. Les idées de CUvier et de LAMARCK fur la classification des. Mollusques me paroissent les plus claires et les plus naturels, c'eft pourquö. je les ai adoptés aussi pour l'arrange. ment de ceus qui font partie de notre Miseum. recentes:

En voici le tableau basé fur les decouvertes les plus.

, apos LINNAEI, Lepades . Echinos, Afteriasque cum Eucrinis colocari dcberc "ecenser. Scrpulae tandem plesaeque, a reliquis test. ccis (qubus test? quasi pro o se , (At, p rtemare corporis. conftimit, ) co maximae diversac, quod tubo non aduarae ,fint, Serpulae inquam, cum Nereide et Aphrodita indolem ct tabican comnunen , hab ut atque in vnam ordimem conjugi debent, qui ad Zoophyta gradum cficis,

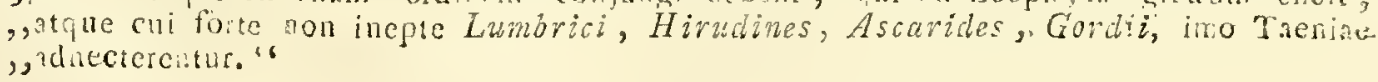


Mollusques céphalés.

ก $\mathrm{u} s$

2. Cellx Gui nas gent.

- Dc longues tenttacules aptour de la tete, des branchies cachées ou interres.

seche.

Colmar.

Poulpe.

Icrinéc

- T'entacutos courtes, branchies ejuternes en forme - dic nogeoires.

Clio

Picumoderme,

Firole.

Dorl is

Tritonie

Glaucus. Eolid

b. $C \in u x q u i$ a $\mathrm{mpent}$ fur unventre laro $\mathrm{g}$ e.

* Tentacules courtes cu nulles, branchies inters nes.

Lalpysie

Limace?

parmacelle.

Onchidic,

Tethys.

Bullees.

Sigarets.

Hhyllidie.

* Tentacules courtes, branchies externes.

šyllée.

Pleurobranche.

conch y lifères.

Coq. anivalve, uniloculaire, non fipirale recouvrant l'animal;

* En forme de tuiles ou d'úcailles réunies por anz contour conzsilusis;

Oscabrion.

- En forme de bouclier... Patclle.

Fissurelle. Emaginulc. Concholepas. Crepidule. Calyptrée. Galerite.
C. univaive, uniloculaire, fpirivalve et singainant l'animal.

6

Ouverturéchan Ouverture enticrée ou canali- ire et fans ca. culié à fa base. nal à ja base.

conne.

Porcelaine.

Orule.

Tarrière.

Olive.

Ancille

Voliute.

Mitre.

Colombelle.

Marginelle.

Cancellaire.

Nasse.

Pourpre.

Bucein.

Eburne.

$V$ is.

Tonne.

Harpe.

Casque.

Strombe.

Pterocère

Rostellaire.

Rucher.

Fuseau

Pyrule. Fasciolaire.

Turbinelle

Pleurotome

Clavatule.

Cerite.
Toupic.

Cadran.

Sabot.

Monodonte.

Clyclostome.

Scalaire.

Maillot.

Turritelle.

Jauthine

Bulle

Bulime.

Amphibulime

Agsthine

Lemnéc.

Melanic.

Pyramidelle.

Auricule.

Volvaire.

Ampullairco

Planorbe

Helice.

Helicine.

Nerite.

Natice.

Testacelle

Stomite

Haliotide.

Vermiculaire.

Siliquaire.

Arrosoir

Carinaire.

Argonauic.
Coq. univalve multiloculaire engainant ou renfermant $[" a$ nimal.

Nautile. Orbulite. Ammonite. Planulite. Numilite. Spirule. Turrilite. Eaculite. Orthocère. Hippurite. Beiemnite. 
Mollusques acéphalés

I U 5 .

c onchylífères.

Ascidie.

Bipbore

M. mmairc.

boule.
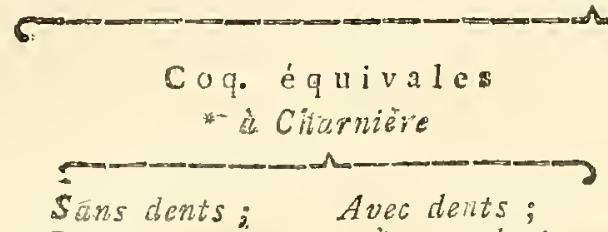

Hunie.

Munle.

Mudiole.

Aliodonte.

Cyrodaire.

Glycimère.

* $\ddot{t}$ un ize desit.

Mulette.

Crassatelle.

raphie.

Mscire.

Myc.
* is derix dients;

Trigoule.

Trilacue.

Hippope.

ciartire.

Lutraire.

Erucinc.

Fétricole.

Vènéricarde.

Solen.

Capse.

Samguinolaire.

* Avec des furazumeraires

Isocarde.

Donace.

Cyclade.

Galathée.

Telline.

$V$ enus.

* à 4 clents

Buccarde.

Méretrice.

lucine.
Cóp. inéquivaIves.

* à Charniere

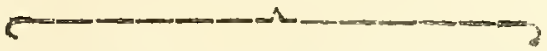

Sans dents. Avec dents.

ficarde. * is uns lest

Radiolite. Came

$V$ ulselle. Corbule.

MarteavoD Dicérate.

Huitre.

-Avicule.

* a doux dents

spondyle.

Plicatule.

Lime.

Houlette.

Cranie.

Hyale.

Luigule

Placune.

Pandore

Térébratule.

Anonie.

Calcéole.

- is piusieurs dents

Peine.

Crenatule. 


\section{$M O L L U S Q U E S$.}

Première fous-division:

Mollusques céphalés.

Ls ont une tête mobile et diftincte à l'extrémité antérieure ou fu* périeure du corps, et. le plus fouvent des yeux et des ten. tacules fur la tête.

$$
\text { Pre } m \text { i e } r \text { o r d r e. }
$$

Mollusques: cephalés nus.

Il ne sont jamais renfermés dans: une coquille à l'extérieur. Plusieurs: contiennent dans leur intérieur un ou plusieursi corps folides, foit cornés, foit crétacés.

A. Ceux quit nagent vaguement dans. les: e a $u x$

* Des tentacules longues autour de la tête, les branchies cachées. intéricieurrement:

$$
\text { Cephalopodes: de Cuvier. }
$$

$$
\text { S èche. Sipia Li no. }
$$

Corpg charnu, deprimé, contenu dans ua fac ailé dans toute fá longueur, efrenfermant vers le dos un os libre, crétacé et rpongienx.

Bouche terminale, entourée de dix bras garnis de ventouses g. et dont deax font: pédouculée et plus longs que les autres.

* Sepia officinalis: Mus. I. 1 .

$$
\text { C a } 1 \mathrm{~m} \text { a } \mathrm{l}^{\circ} \text { : Loligo. I a } \mathrm{mo.}
$$

Corps charnu; , alongé, contenu dans un sac ailé. inférieusement, et renfermant vers le dos: une lame mince, transparente et cortié:

Bouche terminale, cutourée de dix bras garnit de ventouses, et dont deux sont plus longs que les autres.

* Loligo vulgaris M. I. 


\section{Poulpe. Octopus I. a m. C.ur.}

Corps charnu, obtus inférieurement, et contenu dans un sac dépourvu d’ailes. Osselet dorsal nul ou fort petit.

Bouche terminale, entourée de huit bras ẻgaux, munis de ventouses sessilec et aans griffes.

Octopus vulgaris; Sep. octopus Lin.

Le rnée, Lernaea Lin.

Corps oblong, cylindracẻ, renflé au milieu ou vers sa base. Bouche en trompe rétactile.

Deux ou trois bras tentaculiformes à $l^{\prime}$ extrémité antérieure du corps. Deux paquets d'ovaires ou d'inteltins pendans à son extrémité poftérieure.

* Lernaea cyprinacea. Mus. Imp.

* * des tentacules courtes, des brancbies externes en forma de nagcoires.

\section{Pteropodes de Cuvier.}

\section{Clio, Clio. I, in.}

Corps contenu dans un sac oblong, turbiné, muni supérieurement de deux ailes branchioales, membraneuses opposées l'une à l'autr $e$.

Tête saillante entre les ailes, séparée du corps pas un étranglement, formée de deux tubereules entre lesquiels eft la bouche. Deux tentacules courtes insérées sur la tête. Clio borealis Pall. Spic. Zool. IO. 28. to I. f. I8. IV.

Preumoderme, Pnemmodernes Cuvier.

Corps nu; deux nageoires aux cotés du cou; deux panaches de tentacules à la bouche. Les branchies à la surface de la partie poftérieure du corps.

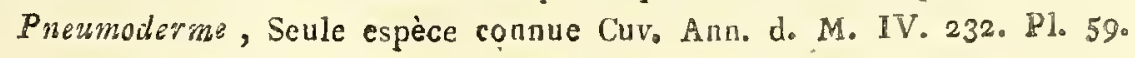

Firole. Pterotrachea Forskäl.

Corps libre, oblong, gélatineur, muni d'une nageoire mobile et gelatineuse, soit sous l'abdomen, soit à la queue; les branchies. .....

Denx yeux apparens fur la tête.

Pterotrachea coronata Forsk. Encyclop. to 88. fo \&o

\section{Doris. Doris Lin. Cuv.}

Deux tentacules en dessus, deux ell dessous d'un manteau pius ou moins étendus an forme de bouclier, et les brauchies autour d'un anus placé fur l'arriêre du dos. $2^{*}$ 
Bouche en uessous vers une extrémité. Anus aul bas du dos a découpé, frange̊ ou cilliẻ fur les bords par les branchies qui $1^{3}$ entouremto

Doris argo Barbut Vers t..4. fo:

Tritonie, Tritonia C ivier. Lam.

Deux tentacules en dessus feulement, les branchies en forme d'arbres plantées le long des deux cotés di dos, et l'anus fur le coté droit. Point do manteau.

Tritonic arborescens. Cuvier An。 du Mus. VI. t. 66. $8-10$.

G la que. Giancus Forster.

Des branchies palmèes píacées des deux cotess comme des nageoires; lianus comme les Trutoules. Point de manteau.

Eolide, Eolides C.u $\mathrm{v}$ i e.r.

Quatre, à 6 tencacules coniques à ha tête; les branchies en forme. d’écailles ca de triltes couchées fur un ou plusieurs rangs le long des deux cotés du dos.

Eolides, nauvelle espéce Cu viex. An. du Mus. V1. pa 430. t. 66. f. I2. 23.

18. Ceus gui rampent fur un large fillon . $\mathrm{d} u$, ventr, e.

Gasteropodes de Cuvier

Des temacoles courtes ou mulles, les brancbies interientrement.

A. I i m a c i e r s.

Aply sire. Aplysia Lin.

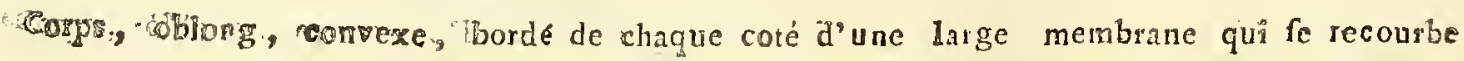

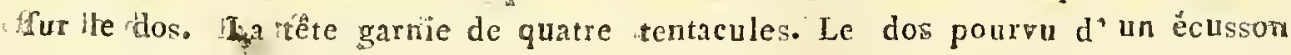
igecouvrant iles 'branchies er comtenant une pièce cormée. L'anus au dessus de - in'extèmité du dos.

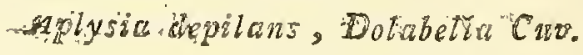

\section{Is im a c e Limax Lin.}

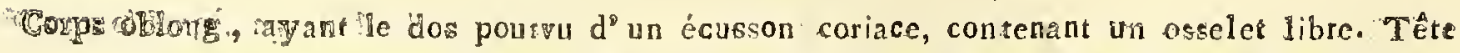
tam unie ide rquater itentacules, dont les deux plus longues portent chacune un oeil

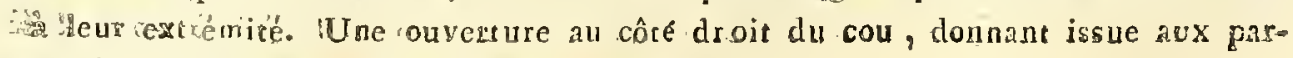

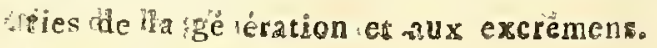

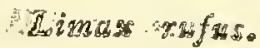




\section{PARMACELLE。-PHILLIDIE \\ Parmaceile Parmacella Cuv.}

ores oblong, partant un manteau en forme de bouclier, plus graind que la eoquille, laquelie s'y trouve cachée et placée plus en arrière par rapport au refte du corps. Parmacelle de Mesepotanize Cuv. Annal. V. p. 435.

$$
\text { Onchidie, Ozbidizin Buch a n nan. }
$$

Corps oblong: Tête munic de deux appertứces âurformes et de deux tentacules. Manteau debordant ézalement de tous côtes.

Bouche antérieure. Anus à l'extrémité poférieure cr en deffors.

Onchidium typhae Trans. of the L1mn. Soc. V. I32.

$$
\text { Tethys. Tethys. I, in. }
$$

Corps ublong, bordé d'un manteau, qui s'épanouit anterieurement et s'étend au dessus de Ia pếte en un voile large, arrondi et frangé.

Buuche salongeant en trompe, et située sous le voile qui couvre la tête. Deus ouvertures au coré droit du cou, pour la génération et la respiration. Tethys fimbria Buhadsch. Mar. 54. t. 45. f. x. 2.

\section{Bulléee. Buillaea. L a marck.}

Corps ovale-oblong, convexe, 'bordé đe membranes qui l'enveioppent. Téte nue, sans tentacules. Partie poftéricure du corps pourvue d'u ácusson large, embrassant, rccouvrant les tratichics, et contenant un corps conchyliforme.

Bullaea planciana Lamarck p. $\widehat{C}_{3}$.

$$
\text { B. Ph y } 11 \text { i d i e } n \text { s. }
$$

\section{$\mathrm{Sig}$ a ret. Sigaretzls. Cuvier.}

Corps ovale convexe, couvert d'un manteau lisse, iniérieurement conchylifère, et qui le tićborde tout autour. Bords du manteau vasculeux en dessous.

Téte applatie, située sous la partie antécieure da manteau, et munie de deux tentacules courtes.

Sigaretus hatiotoideus, Hel. haliot. Lin.

$$
\text { Phylli i die. 'Pbyllidito "C u vier. }
$$

Corps ovale-oblong, rampant, convexe en dessus et couvert d'un écusson ou manteau coriace variqueux, tuberculeux, qui le déborde par tout.

Branchies disposées en feuiliets meubraneux, placés à la file les uns des alutres autour du corps, sons le rebord du manteau.

Phyllidia trilineate Cuvier Ann. du M, v. 266. Pl. 18. 
Scyllée. Scyllaea Linné. Cuvier.

Corps oblong, presque cylindrique, avec une longue queue pointue; bouche à l'extrémité antérieure, accompagnée de tentacules non resractiles; anus latéral; trois pisires dc branchies sur les côtés.

Scyllaea pelagica Cuv. An. Vl. 42 r.

Pleurobranche. Pleurobranchus Cuvier.

Corps ovale, picds ausi large que son mantcau, présentant comme deux boucliers qui ne sont séparés que par un sanal qui fair le tour du corps. Bouche en avant en forme de trompe; branchies du coté droit enlame longitudinale, portant des deux côtés les séries transversales de vaisseaux.

Une petite coquille platte mince, ovale, oblique, blanche, et composée de couches à l'intérieur sur le péritoine.

Pleurobranchus Peronii Cuy, An. V. 269. t. I8. I.

\section{$D E U X I E M E S E C T I O N$}

Mollusques cephalés, extérieurement

c o n c h y 1 if ér es

$\mathbb{P} E \mathbb{E} \mathrm{I} E \mathrm{R}$ O $\mathrm{D} R \mathrm{E}$

C o quille r e c o u v r a n te

Coquille univalve non spirale, recouvrant simplenert l'animal.

* En forme de tuiles ou d'écailles réunies par un ligament circulaire.

$$
\begin{gathered}
\text { Oscabrion. Chiton Lin. } \\
\text { Lophyrus Poli. }
\end{gathered}
$$

Corps ovalc-oblong, rampant, convexe en dessus et couvert d’un manteall qui déborde de tous côté pièces teftacées imbriquées, transverses, eachassćes dans son eparaeur et plus ou moias apparentes au dehors. 
Les branchies placées sous le rebord du manteau rout avtour du corfs, formen une suite de petits fcuillets vasculeux rangés a la file les uns: des autres.

T. Oscabrion bicolor, à huit valves épaisses, le dehors vert, le dedans blanc, et le bord noir.

Chiton bicolor, tefta octovalvi, crassa dorsaka, thalassina intus nivea margine nigra. La. Gm. 3204. I0.

Chemniz Conchyl. 8. t. 94. fo 794.

pays natal inconnu. 2 p. 4 lignes de largeur étant courbé, et I p. 7 1. de long'seur.

2. Variété du même généralement vert, plus alongé, à écailles intélieurement decoupées. Les: écailles. latérales alongées bifides.

3. IL même couvert d"une couche calcaire ferpulifère:

L'oscabrion cerasin, à huit valves unies, couleur de cerises; le bord avec des dents blanches.

chiton cerasinus, tefta octovaivi cerasina laevi : marginis: dencibus niveis. Ln. Gm. 3204. II ${ }^{\circ}$

Cherinitz Conch. 8. t. 94. f. 796.

Le nôtre diffire ccpendant en ce que $1^{\prime}$ intérieur eff pe' $\mathscr{E}^{\prime}$ et que leś écailies présenreut des Interftices et ant aussi découpécs. II a 2 po 5 lo de large, sur \& $p_{0}$ de long.

5. * L"oscabrion de $\mathrm{ex}$-points, à huit valves ridées; les. valves terminales sillonnées; les intermédiaires garniss de coté et par devant de trois points élévés.

Chiton bipunctatus, tefta octovalvi: rugosa', terminalibus sulca. tis , intermediis: latere margineque anteriore bipunctatis

Cette espèce d'Oscabrion, couverte d' une' croute calcaire, sans être fossile, eft: nouvelle. Elle eft de la grandeur de la précedente ${ }^{\prime} ;$ fes: écailles; font ridées, les terminales fillonnées; les fillons: fortent d' un centre commun et fe terminent avant le contour dans un bourrelet élévé: Les écailies intermédicirres présentent auprès de leur fommet. qui fe: termine en épine élevée, deux points noirs et à la 
marge opposée, de coté, trois points noirs. élévés. L'intẻrieur eft $d$ 'un vert très foncé. l.es contours font blancs, garnis d’écailles grisâtres en forme de lentilies.

I.e pays natal m'en eft inconnu. Le port total le diftingue de lOsbabrion tacheté de Chemnitz 8. t. 95. f. 802: 2. p. 2. 1 . de longueur; 1. p. 11 . de largeur.

6.. L'oseabrion ftrié, ì huit yalves très śpaisses, rougesbrun, ftriées de blanc.

Ckiton ftriatus, sesta octavalui crassa, ex rubro, bruneo et albo - ftriata.

Un bel individu, gui convient $3: 5 e z$ a 13 défriptiondu Chiton crassus friatus de (hemnitz 8. p. 280 . fans cependaur s'ascorder avecla figure qui cu donne Tab. 95. f. 80I. Il eft d'un rouge brun; au milieu, auprès de la caréne, pius

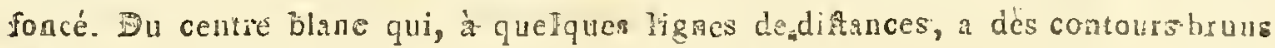
ess oval, fortent des. lignes blancheg en divergeant vers le contous latéral. Celx de la yartic antéricure qui fart l'articulation mutuclle, font placées paaallelement les uncs à cotés dez autres. Toutes les lignes examinées fous la Ioupe, présertent des points élévés réunis en chroclet.

Certe belle espèce mérite une meilleur figure. Ellc a 1. p. 6. lignes de largeur fur \& lignes de longueur.

7. 8. * l'o c a brion ondulé, à huit valves lisses branes, a firies transiersalos, ondulées jaunes,

Cbiton undailatus, testa octovalvi laevi brunnea, ftriis transversalibus vndulatis flavis.

Les deux exemplaires paroissent apartenir đa la mêne espère quoique l'une foit un peu plus grande et même plus pâle en couleurs. Les ftries transversales fe font plus près au milieu de la quatriéme et cinquiéme valve; là elles imitent des flammes. La ligne du mílieu qui fait le centre ou le fommet ef grisâtre accompagnée d'une bordure branefoncé. Le ligament cî́culaire eft garni de petites écailles alongèes grîses et verté. Le's écailles ou valves principales font intérieurement dentélées et d"un vert très pâté.

sune a 1. p. 5: 1. de largeni, fur 10. l. de longueur et Saucre 1. p. 3. 1. de Farge, fur 9 de long. 
9. toscabrion incomplet, à huit valves blanckes jaunâtres qui ne touchent pas le contour ligamenteux.

Chiton incompletus, testa octovalvi laevi, ligamentum circulare non attingente.

Cet oscabrion a 1 p. 2 1. de largeur fur 8 1. de longneur. Les valves font partout bordées $d^{\prime}$ un large contour mem. braneux où on ne voit point de trace des écailles latérales qui laissent toujours des impressions plus ou moins pro. fondes. Les valves elles - mêmes font james avec des lignes concentriques violettes pâles. Le fommet forme une ligne élévée brune à trois sillons bien profonds des deux cotés. Les deux valves terminales sont rayonnées, à rayons divergens formés par des points, bruns et imprimés. On tiouve de pareils rayons sur les valves intermédiaires qui partent du fommet vers le contour. İ'intérieur est blanc-rougeâtre et les valves sont un peu découpées au milieu.

* * en forme de bouclier;

Les mollusques de cette sous - division ont les branchies placées à nu tout autour du corps sous le rebord du mantoau et appartiennent par conséquent ì la famille des phyllidiens.

$$
\text { patclie. Patolla Lin. }
$$

Ceq ovale ou suborbiculate, en bouclier ou en bonnet, concave et simple en dessous, entiere a son sonmet et sars fissure à son bord.

Anim Gaserupode de têic tronquée obliquement munie de deus icntacules poinrues. Lis yeuz à a base cxtéricure des tentacules. Les branchies placécs"autour da corps sons le rebord du mantean.

* Celles dont la base est anguleuse ou dentée.

3. L'oeil de r ubis, blanche avec les bords tachetés de brun en zijzags; le sommet brun, entouré de cercles de diverses couleurs, les stries nombreuses, épineuses.

$\mathrm{P}$ t ella granat ina Lin. Gm. I. 6. 36, 6. 22.

Martini Conclyyl. I. 9. $71-74$. 
2. 3. Deux a u res à sommet moins élevé.

4. 5. O e il de rubis d'un jeune animal, et un autre à sommet élevé et très vif en couleurs.

6. Le grand oeil de bouc, brune, à stries armées d'epines blanches imbriquées.

Patella granularis, Lin. Gm. I. 6. 36g6. 2I.

7. Patelle sanguine, brune ou rouge-clinire en dehors et en dedans; les grandes côtes alternant avec les petites; le sommet applati blanc.

Patclla sanguinca Lin. Gm. 1. 6. 3705. 62. Bosc. Coq. 3. 2òr.

Notre exemplaire a 3 p. er demi de longueur.

8. Patelle deux yeux, Rayons élevésingaux, l'inter évalle des rayons striés transversalement; le sommet à deux tubercules blancs.

Patella laciniosa Lin. Gin. I. 6. 3695. 4.8. Bosc. 3. 192.

9. Patelle à côtes, ovales à côtes élevées et comprimées, blanches inégales, taberculeuses.

Patella costata Lin. Gin. 1. 6. 369g. 33.

10. * Patelle à I I côtes, blanche ondulée de brun, à I. côtes élevées principales touchant avec leurs racines le sonimet.

Patella undecim - costat a mihi.

La Patrie en est inconnu; I p. 7 l. de longueur, 1 p. 31 . de largeur.

II * Patelle bifide, ovale, blanche à bandes oranges, à sommet élevé à côtes rapprochées bases; les côtes du bord allongées comprimées, bifides.

Patella bifida mihi.

On ignore sa patrie. Elle a 1 p. 6 1. de longueur fur I p. 2 1. de largeur fans les épines.

12. Le Soleil de Cythère, blanche, marbrée de taches noires anguleuses avec fept cannelures. 
Patella ia echarina. Lin. Gin. 3605 , 17. 19.

Martini. 1. 9. 6. $70.75 .76 .7 \%$

Bos:. 3. 192. de Roi sy. 5. 214.4.

Vulgairement: $l^{\prime}$ 'étoile dis anatin.

13. * La digi té e, brune, rayée de blanc, à I I côtes élevées arrondies, depassant de beaucoup les bords.

Pasella digieata, m.

Elle eft à l'intérieu: blanche nacrée, bordée de brun.

14. L'é p i ne use, blanche, à fornmet et à rayons oranges; à I 2 cốtes élevées très tranchantes, depassant de beacoup les bords avec des pointes tranchantes.

Patella rpinosa, m.

15. * P. à 7ra yons, anguleuse avec-fept côtes rayées transversalement.

Patella feptem-radiata, m.

Cette patelle est blanche-jaunâtre, bigarrée de brun. I e fommet et les bords font d'un brun uniforme. Entre les rayons principaus fe trouvent cinq ou fix cordons élévés d'un blanc nacré.

On ignore fa patrie.

I6. - $18 \mathrm{Grand}-\mathrm{Sol}$ eil deponillé, l’un deprimé à beau volume, le troisieme portant un balane. d'Argenville. I. p. 513. Patella radiata.

19. 20. Petit foleil depouillé et nacré.

2I. Petit soleil couvert en partie d'une croute calcaire blanche.

22. Patelle depou illé e et à ce qui praroit, un jeune exemplaire de l'oeil de rubis.

23. P a telle radié e couverte de fucus et de corallines.

24. * Patelle leucogramme, ovale à fommet pointu, bleu d'indigo et presque central, à I 2 côtes élevées blanches. Patella leucogrammi. mili, refta ovali tenui, ftriis elevatis albis vndecim, vertice mucronato azureo, Ia $2^{*}$ 
patrie en eft incomnue. Le dedans eft blea $d$ 'indigo au fond qui est ceinturé de hrun. Ce $n^{5}$ eft que le bord qui présente à l'intérieur des raies blanches. Cette belle patelle a 9 lignes de longueur sur 7 de largeur.

25. 26. * Patelle scie, presque ronde, à côtes nombreuses élevées et dentélées en forme de scie.

Patella, serrata mihi, testa sub-rotunda, costis multis elevatis serratis.

Deux exemplaires dont l'une est verte et rouge, rayée de blanc à l'intérieur, l'autre blanche et jaune.

Elles $n$ ont que 8 lignes de longueur sur 6 de largear, et paraissent avoir quelque analogie avec la radiée, Patella alboradiata de Bosc. 3. 199.

27. Patelle rayé é elle paroit un jeune individu de la b if id $气$,

$28-31$. Le bouclier couleur de rose, ovale, bord crénelé rouge rose àvec des stries élevées blanches.

Patella cosea.

d'Argenville. I. 488. pl. 1. H. T.

Quatre variétés présentant différentes nuances de la couleur rouge. - De lista de France.

32. 33. L e boucli er lose, deux variétés dont les stries blanches sont plus élevées.

34. 35. Deux variétés du même dont le sommet est rouge mêlé de jaune.

36. 37. P. magella $\mathrm{n}$ ique, ovale, mince blanche; les bords noduleux; le dedans nacré; les côtos élevées jaunâtres; le sommet aigu.

Patella magellanica. Ln. Gm. 3703.5a.

Martinio I. 5، 40. a. b.

Du detroir de Magellan.

35. I a même garnie de balanes.

39 - 50. I a $m$ ême en I 2 variétés resultant de l'art de depouilier les coquilleg 
51-56. La m $\mathrm{e} m$ e en 6 variétés de la plus belle dorure.

57 - 59. Deu $x$ bo a cliers nacrés du même detroit appartenant à la même espèce.

6ó-62. Trois entonnoirs depouillés et nacrés du méme endroit préserrtant de jemes cxemplaires de la même espèce.

63. Entonnoir avec deux bandes blanches, is oeir de grenat qui paroit furmer avec les patelles précedortes la mene espèce.

6 fo Patelle br ún e á 20 stries élevées noduleuses, à sommet rougie de grenat, à intéricur blanc.

Elle paroit ure variété des précédentes. Elle approche par ses stries nedulcuses de la patelle nadulosa Lin. mais elle en differe gar la colteur du sommet. qui est blanc dans la dernière.

65 - 6. I a c el le froncée, ovale, rugueuse, châtain; le scmmet gris, le fond comronné de blanc, $l^{r}$ intérieur cendré, sadié de blanc: le fond brun pâle entouré de blanc.

Patella c orrugata, Lin. Gm. 3702. 44. Bose. 3. 197.

Cinq beatux échantilons qui d fïzent par les nuances du depouillement.

7o. Patelíe rouillée, couleur de rouille, avec trois fascies jaunes, les stries êlevées inégales, aignës? le sommet blanc (jaune dars la nôtre); le fond nacré.

Patella ochroled ca a, Lin. Om. 3703. 53. Bose. 3. 599.

71. 72. Patelle orange, ovale, citrine, ondée de brun, les stries les plus élevées rapprochées, rugueuses; le fond blanc.

Patella auratia, Lin. Gm. 3703. 49. Easc. 3. Ig8o

73-76. Patelle seriatule, un peu rugueuse, blanche avec des stries brunes, flexueuses, ramifiées à l'extérien; l'intérieur ayant deux taches brunes.

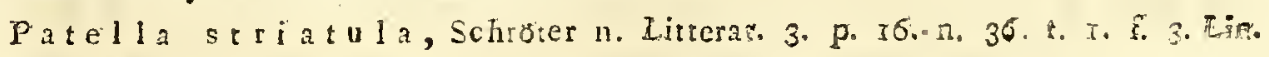
Gm. 3699 . 35. Bosc. 3. 195 . Quarre échantlions da differente grandeur: 
7\%. 78. P a te $11 \mathrm{e}$ o liv a cée, ovale, couleur d'olive, en dedans biun variée de blanc; les côtes inégales, bordées de deux rangs d'épines obtuses, le fommet jaune, le fond nacré, le centre brun

Patella olivacea, Schroeter Einleit. 2. 490. t. 6. f. 1. Lin. Gm. 3702. 45. Bosc. 3. 198.

79. Patelle couleur de cire, ovale striée en dedans et en dehors par des lignes couleur de cire; treize côtes applaties, le fond blanc

Pat ell a cere a Schrocter, n. L. 3. 59. 110. Lin. Gm. 3702. 47. Bose. 3. 198. 80. Fatelle bè ge, peu convexe brune; l'intérieur jaune paille; douze plus grands rayons accompagnés du même nomb̧re. de petits.

Patella badia, Schröter Ein1. 2. 484, to 5. f. 9. Lin. Gmel. 370039. Bosc. 3. 196.

8I. 82. Patelle bè ge, variété qui est à l'intćrieur olivâtre rayée de jaune.

83. 84. Deux Patelles nacrées qui paroissent apartenir à la même espèce.

85. * Patell e à côtes rouges, ovale, peu convese jaune, bordee de brun, à 16 côtes rouges.

Patella rubro-costata, testa ovali, subconvexa, flava, badio maculata, costis sedecim elevatis rubris.

Le dedans est rouge rose, rayé de brun aax bords, le fond est blanc, comme le sommet; elle a 10 lignes de loigueur sur 8 de largeur.

On ignore soll lieu natal.

86. 87. * Pat ell les à cốtes rouges deux variétés par l'élevation et le nombre des cótes.

88. 89. * Patelle double-rayon, ovale, blanche, des rayons élevés nombreux noires avec des intermédiaires plus. courts rouges - pourpre. 
Patella dublo-radiata, testa ovali, alba, costis numerosis elevatis nigris, intermediis brevioribus purpu. rascentibus.

Le sommet est blanc ceinturé de brun, le dedans blanc; le fond est noir et blanc de sorte que le noir imite assez bicn le contour d'une tête d'idole d'Egypte. L'une a 1 pouce de longueur sur 10 de largeur; l'autre a 8 lignes de long sur 8 de large. Num patella notata Lin?

Son lieu natal n'est pas connu.

90. Pat ell e plus convexe, rayée de brun, dont l'espéce ne se laisse point determiner.

91. Patelle varié e, ovale, un peu ànguleuse, obscure, les côtes inégales, unies, rapprochées, blanches.

Patella leucopleura, Lin. Gin. 3699. 34. Bosc. 3. I95.

92. Patelle costée, ovale un peu bossue, avec des côtes blanches, inégales et tuberculeuses.

Patella costata, Lin. Gm. 3699. 33. Bosc. 3. 195. un très petis exemplaire.

93. 94. * Patelles à neuf rayons, ovale, bleuâtre, à neuf rayons.élevés blanıs,

Patella novem-radiata, testa ovali, tenui, pellucida caerulea, costata, novem costis albis.

Le sommet eft bas $\in t$ jaune, entouré de blanc, le fond à l'intérieur est brun, le reste est bleuâtre avec des stries jaunes.

95. Patelle pentagone, blanche, obtuse, pentagone; le bord dilaté, crénelé, le sommet obtus; le fond rougeâtre.

Patella pentagona, Eorn Mus. caes. vind. t. I8. f. 4. 5. Lin. Gm. 3708 . 84. Bosc. 3. $2 \subset 6$.

96. 97. P. petite mitre, epaisse, presque conique, plissée dans sa largeur; le bord siılueux. 
Patel lamitrula. Lin. Gm. 3708. 82. Rosi. 3. 20g; de Boissy. 5.216. I4. Lister tab. 544: f. $3 \mathrm{~F}^{\circ}$ Martini. r. t. 12. f. JI. I. 12 .

De la mer des Antilics.

Les trois dernières espèces me paroissont ćtrangères à cette division es ă ce genre.

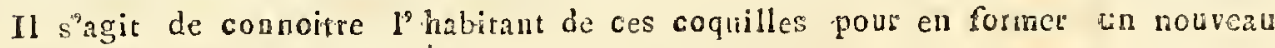
genre. La mirre sapproche davantage des Galérites.

$97^{\circ}$ Pat. peig $\mathrm{ne}$, orale radiée de blans, les rayons écartés, pectinés à leur extrémité; le sommet gris, le fond couron. né par une tache entourée de blanc.

Pate l-1 a pecten, Lill. Gm. 3702. 43 .

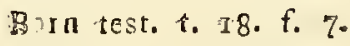

Quatre exemplaires; dont le plus grand jaure, les autres zleuatres.

Ces dernières paroissent former une esjèce distincte par les rayons intermédiaires non pectinés.

* Base simple et non dentée.

98. Pat. boucIier, aigue, unie, très glabre; fauve marbrée de brun rougeâtre.

Patclla testudinaria, Lin. Gm. $37 \%$ r 34. Bosc. 3. $27 \%$

de Roissy. 5. 217. 15 .

Dargenville t. 2. f. P.

Vulgairement l’écaille de torlue, dans la merdu Nord et dans celle de l'Inde.

99. Le mê m e bouclier nacré au sommet et d'une grandeur considérable; si ce $n^{\prime}$ est pas une espèce distincte.

I00. La test u $d$ i n a le, ovale, strite. Celle-ci est nacrée.

Patella testudinalis. Lin. Gm. 3717. rít. Bosc. 3. 2 r6.

IOI. I e bateau, ovale comprimée des côtés, jaune, à rayons élevés, à sommet blanc ponctué de rouge écarlate.

Patella compressa, testa ovali lateribus compressis, fulta costata, vertice albo rubro maculato.

Cetre belie coquilic éroit dẻjà connue à $\mathbf{L}$ isı er, Conchyl. t. 54. 25 patella magna, fulva, lateribus pauluium compressis, dense ct lauter striate; mais quelques areus tels que $G$ melin 3718 . 136. de Roissy $5 \cdot 2.8$ 21 et autres, confondent deur espèces, ce qui prourent par les citations destigures. 
La tigure de Ma'ri i i convient d une coquille deprimées qui avec celle - là 5.'a de commun que la couleur jaunâtre. Notre exemplaise eft de belle grandeur et de vives couleurs. Elle a 3 pouces de longuear, et dans toute sa largeur qui est aniforme par la compression des cotés, I pouce, Le dedans est jaune et le fond blanc-nacré.

On ignore son lies natal.

102 - 104. P. F l a m bée, ovale à rayons ondulés bruns. Le sommet est brun.

Patella fla m inea, de Rojssy. 50218. 19. Trois exemplaires différem ment depouillés.

105 - r06. P. pa p y a c ée, applatie, mince, demitranspare. te, ponctuée de rouge, radiée de fauve; les petites stries réunies, les grosses granulées.

Patelia papyracea, Schroeter Einlo 2 p. 487. to 5. f. I2. Lin. Gm. 3722. 158.

I07. * P. t a chetée, ovale, deprimée, brune-foncé, lisse à côtes élévées, tachetées de blanc.

Patella maculata, testa ovali, depressa, fusca, maculis albis irregulariter conspurcata.

Cette belle coquille a le sommet brun plus claire, placé un peu plus en arrière, le dedans est blanc; elle est très épaisse et paroît composée de trois couches. Elle a I p. I l. de longueur et $10 \mathrm{l}$. de largenr.

Du decroit de Magellan.

109. 109. P. f a u ve ovale, déprimée, finement striée, a sommet placé un pezi plus d'un coté.

Pa c clla depressa; $c^{9} t s t$ a cette coquille quappartient la figure de Martini I. 52. 106. et celle de la patella lutec de Bozn. Test. Tab. I8. f. 8 .

Iio. P. c o m primée, ovale, comprimée, jaune, à fommet er mamelon.

Cette fatelle paroît un jeune individu de la patelle bateau de n. Ior. quoique ic sommer ne soit pas entouré de points rouges. 
III. P. transparente, presque cvale, bossue, tramsparente avec quatre rayons bleus.

Patella pelluc ida. Lin. Gmel. 375\%. 333.

Born. Testac. E. I8. f. 9 .

Chemnitz. I0. t. I68. f. I620. 1628.

II2. P. pustullée, mince ovale, applatie, radiée de blanc es ponctuée de rouge.

Patella pustulata, Lin. Gm. 3720. 147\%

I $3-116$. P. a r réole, ovale, applarie blanche avec des rayons en auréole, qui se multiplient vers le bord.

Le guatrième individu de cetre espece est gluis petite et presque plate, le sommet est blanc ceinturé de jaune.

17. P. chinoise, ovale, jaune, épaisse au sommet, mince et tranchante sur les bords; onduleusement sillonnée en dedans; perpendiculairement striée.

Patella sinica; de Roissy. 5. 219. 25. Operculatum lave Lin. Muso Tessin. 6. 6. fo 5. p. $y \rightarrow 6$.

Le parasol chinois, vulgairement, de Favanne Conch. I. t. 3. fo 5. p. 524.

Chemair. 10. p. 34r. t. 169. fo 3645 . 46. la figure est mauvaise.

Pate 11 a u mbellara Lin. Gm. 3720. 146. Bosc. 3. 218.

Cette coģuille est extrémement rare et vient de l'isle de france.

* Patelles fossiles.

[18. Grande parelle à cotes mais altérée à la surface de sorte qu'il est impossible d'en definir l'espèce. Elle eft couverte de corallines.

19-121. Trois pat elles à côtes.

Voy. les pacellites du $M$ us e u $m-D$ e midoff vol. II. p. 266. n. $28-49$. 
Fissurelle. Fissurella Itam.

Coq, en boucilier, sans spire quelconque, concave en dessous ef percée au sommet d'ua trou ovale ou oblong.

Animal. Gastéropode ayant le disque rentral frangé et la frange du bord du manteau composée de filets anueux. Anus sortant pis le sommet de la coq̨uille.

I I2. Fissurelle radiée, ovale solide, variée de blanc sale ou de vert obscur; les rayons obliques, ondalés alternativement violets et blancs.

Fis 8 ugella sadiata; Lam. Arim, fo vert. po eg. Patella picta Lin.

Martini I. It. 90 .

Ces iz excmplaires dont phusieurs d"una beise grandeur présentent diffèrentes Enances de couleus; - du detroit de Mugellan, et d.s isles Malouines.

8- I3. Fiss. trou de Serrure, ovale, striée, rugueuse brune; le trou du sommet oblong, légèrement étranglé dans son miliea. -

Fis surella nimbosa de Roissy. 5. 230. 3 .

Patella nimbosa; Lin. Gmo 3729. 196. Bosc. 3. 228.

14. Fiss des $\mathrm{B}$ arbades, oblongue, inégalement striée; le bord crénelé, en dedans fasuié d'un vert blanchâtre; le trou entouré d'wa cercle fauve. - Le trou conique.

Fissure:la barbadensis; - Parella barbad. Lin. Gm. 3729. I99. E sc. 3. 22.9. - de la mer des Antilles.

15. Fiss, treillis, ovale, convexe, striée en sautoir; le bord crénelé en dedans; le trou ovale près du bord postérieur.

Fissurella grieca, da Rnissy 5. 230. 2. Patella graeca Lin. Gas.3728. 105. Bos 3. 228. - - des côtes d"Afrique.

16. Fiss. de ia $J$ am a iqu ue, blanche, annulée; les stries longicidinalss, avec des tubercules foliacés; Ies plus grands rénis: - le trou arrondi.

Fissurelia Jamaicensis. - Parclia fam. Lin. Gm. 3730, 200. Bosc. 3. 22y. Wariui. .. to I. 1.g. 9\%. - de la mer cies Antilles. 
I7. Variété de la même à côtes plus rouges et lisses.

18. I9. Fiss. nodule use, ovale, un peu convexe; les stries élévées, rugueuses, coupées par de plus petites; le sommet noir; le dedans blanc; - le tron tuberculeux au milieu.

Fissurella nodulosa; - Patella nodulosa Lin. Gm. 3732. 209. Bosc. 3. 23r.

20. Fiss, porpliyre, oblongue, comprimée, inégalement striée, blanche, avec cinq cercles interrompus pourpres; le dedans d'un blanc verdâtre, avec un cercle rouge. - Le trou subtriangulaire.

Fissurella porphyrozonias; - Patella porphyroz. Lin. Gmo 3730. 203 .

25. Fiss. épineuse, ovale, convexe, blanche; les stries les plus élevées plus épaisses à leur extrémité, avec quatre rangs de tubercules; les tubercules extérieurs épineux; le sommet rose; le trou crénelé.

Fissurella spinosa; - Patella spinosa Lin. Gmo 373r. 207. Bosca 3. $23 \mathrm{r}$.

22. Fissurelle à rayons élevés blancs, ¿̇ intermédiaires et à sommet couleur d'olive, paroit une petite variété de la fissurelle treillis.

23. Fiss. rose, ovale, comprimée, à stries rapprochées, alternativement tachetées de rose et de blanc, le trou ovale.

Fissurella rosea; - Patella rosea Lia. Gín. 3730. 204. Bosc. 3. 250 o Marrini 1. t. I2. fig. 105.

24. Variété de la précédente; elle s'accorde presque mieux avec la déscription de Fissurella lactea de Lin.

25. Fiss. rougeàtre, ovale striée, rougeâtre; une fascio, et le dedans blanc; le bord entier, le trou linéaire?

Fissurella rubescens; - Patella rubescens Lin. Gm. 3732. 274.

26-138. Variétés de la même soit dans la conleur soit darns la grandeur en I 2 individus. 


\section{$G$\begin{tabular}{lllllllll}
\hline & $A$ & $L$ & $E$ & 1 & $T$ & $E$ & $S$
\end{tabular}}

Fissurelles fossiles.

139. Fiss are $11 \mathrm{e}$, lisse, ovale, couverte de terre calcaire blanche et des serpules.

140. Fissure 11 e à côtes couverte de terre calcaỉre et de petités balanes. Elle paroit être Fissurella nizmbosa.

Galérite, Galerita Brongni ard.

Coq. à somnet recourbé sur le coté et quelque fois spirale, limbe de leur base irségu• lier, et non pas sur le miême plan.

Animal. inconnu.

5. 2. G. bon net de dragon; Entière, conique, striée fommet aigu, recourbé; blanche ou rose sale; l'intérieur couleur de chair vif. -

Galerita hungarica; - Patella hugarica Lin. Gin. 3709. 89. Bosc. 3. 206. de Roissy. 5. 22I. 27.

Martini I. 12. fo 107. 108. - dans la ner mediterroné Deux exempiaires roses de belle grandeur.

3. 4. Deux a utres plus petirs.

5. 6. Deux bonnets de dragon blancs de la prémière grandeur.

7. Un a utre plus petis à stries plus élevées et à rides plus profondes.

8. Galérite antique, entière, oblongue, imbriquée, le sommet placé en arrière et recourbé.

Ga lerita antiquatí; - Patella antiquata Lin. Gm. 3709. g0. Notre individu n'est pas fossile, mais garni d'an byssus qui remplit les interstices des imbrications. Je crois qu'il faut en faire une nouvelle espece.

9. * Ga lérit e ponctuée, entière, sillonnée par des ligntes ponctuées, le sommet courbé dans le même plan.

Galérita punctata, mihi. - Elle est en dehor's couleur de rose, dedans blanche. J'ignore son lieu natal.

10. Galérite corne d'abondance, entière, obliquement conique, presque rugueuse, base ovale, sommet crochu. 
Galérita cornucopiac.

Patella cornucopiae Lamarck innal. त. M. I. 3r. 5. integra, oblique conica, Gubrugosa basi ovata; verice simeo. -... f. 4. a. b. c. No 33. V. p. 214 Pl. 43. de Roissy. 5. 222. 3I. fossil a Grignon près de Vexaille.

IF. G. f piriroftre; tntive bliquement conique, ftriée Iongitudinalement et ruftiquće, base dilatée formet courbé en spirale fur le coté.

Gajerita spirisostris; - Patelka spiriroztris de Lamerck Annal. d. Mus. 1. 3I. 17.6. de Roissy 5. 222. 32.

La nôtge n'est pas fossile.

Emarginule, Emarginäi Ia marck.

Eoquilie. En bonclier conique, à scmmer inclité, concave cn dessous; bord fendu ors écliancié.

Animal. Inconu; probablement analogue à ceiri des patel'es.

Emarginula conaca Lar. - de Roissy. 5. 232010 - Patcllo fïssura Lisu.

\section{Crepidule, Crepidula I, a m a rck.}

Coquille; oviale ou oblongur, convere en dessus, à sommet incliné sur le bordo La câ vité interrompue partielieinent par un diaplarague simp'e.

Animait. Gasteropode à tềc munie de deux tentacules qui portear les yeux un pell au dessus de lenr basc. Le reste de l'orgatisation a rappost avec celle dos patelles.

1. Crepidule porcelaine, ovale, blanche tachetée de brun rouge; le sommet recourbé, la cloison applatie.

Crcpidula porcellana Lamarck; - de Roiscy. 5.23\%.r. Parella porcellana Lin. Gmel. 3502. 4. Eoso, 3 .189. - Adariso:a, pl. 2. f. 8 . Des mers de l'Inde.

2. Crepidule voutée, ovale, avec des rayons sur le dos, et des taches latérales d'un janne fauve; le sommet decourbé obliquement, la lèvre concave et postérieure. 
Crepidala fornicata, de Roịsy. 5. 238. 2. - Parella fornicha Lin.

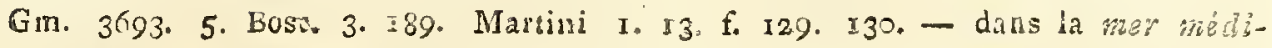
terranée.

3. 4. C. J e n a c, orale, applatie, mince, blanche, bullante; l'exté rieur lamelleus.

Crepidula goreensis, de Roissy 5. 238. 3. - Patella goreensis Lin. Gmel. 3694. Io; Bose. 3. I90. Adanson pl. 2. fo 10. - Sur les rochers de l'isle de Gorée.

5. C. garnot, ovale aplatie, unie, presque transparente; blan. che ou brune, avec deux bandes foncées; cloison plane er très enfoncée.

Crepidula fasciata de Roissy, 5. 238. 4. - Patella crepidula Lir. Gm. 3695.17. Bose, 3. 192. Gualtieri t. 69. f. H. des côtes du Senegal.

6. \%. * C. haliotoüde, ovale, aplatie, à sommet latéral et couché (comme dans les hatiotides) à côtes élevées et granuleuses, à cloison sillonnée.

Crepidula holiotoidea, testa subovali, vertice laterali depresso, striis elevatis granulatis divergentibus, labio suirato.

Cette coquille est très rare, et point d'auteür n'en a fait mention. Elle s'approclie par la forme génerale des haliotides, mais la cloison intélieure la range parmi les crepidules. Cette cloison est sillonnée et échancrée sur le bord. Elle a dix lignes de longueur sur 8 de largeur. - Ie lieu natal en est inconnu.

8-I5. Cr. Sole, legèrement contoumée, transparente, tarhetée de jaune, foiblement plissée; la cloison à bords sinueux.

La nôtre paroit avoir perdu sa cloison, mais il faudra peut être la senvoyor ăt. genre patelle.

\section{Calyptrée, Calyptrea Lamarc Patellae labiatae $L$ i n n a e $\dot{I}$.}

Cuquille. Contide à sommer vertical, entier et eu pointe. La cavirée inrfrieare mngrảe

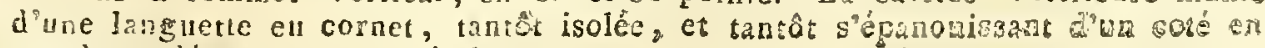
une lame décurrente en spiraile.

Animals inconau. 
* Cal y ptré es à languettes en cornet.

1. Calyptrée clocbet te, vulgairement le bonnet de $\mathrm{N}$ e ptu ne orbiculaire, blanche, presque transparente, irrégulière en dehors; la languette perpendiculaire et centrale.

Calyptraca equestris Lamarck, po $7^{0}$ - - Raissy. 5. 243. I. - Pattella equestris Lin. 369r. I. Marini. I. t. 33. f. 11 \%. II 8. de la mer des Indes. Exenplaire de la plus grande sareté par la grandeur et la bonne coservation.

2. 3. D e u x a u t res plus petites à côtes élevées très aigues et épineuses; - peut-être une espèce diftincte.

Dans ces trois individus la languette intérieure eft un peu adosées de coté, fon origine.

4. 5. C a l y p.tré e i n ég a l e ou chiffonnée, irrégulière, à bord finueux à furface rugueux, à languette partant librement du centre.

Deux exemplaires; dont l'une plus grande, blanche, transparente. Rare et fans doute une espèce distinctc.

6. 7. Calyptrée à ét ages, blanche, à quatre ou plusieurs réplis feuilletés par étages. Ia languette centrale courte et mince.

C a l y ptrea i m bric at a mihi.

L in né en a fait une variété de la Clochette (Mus. Reg. Vir. p. 687. n. 408. Patella labiata equestris a) lamellis horizontalibus imbricatis.) Mais clle doit former une espèce distincte parcequ'elle n'a rien de commùn avec la clo. chet te que les caractères génériques. C'est une des plus rares coquilles. V. Mattini. I. 155. t. 13. f. 125. 126. Nos deux exemplaires font parfaitement bien conservés; l’un, plus grand, à quatre étages plus difrantes; l’autre, plus petit, en a fix qui font ondulées.

* * a l y ptré es à languette en lame décurrente en ipirale.

8. 9. Calyptrée toît chinois, conique, unie, blanche, la languette latérale.

Cal 7 ptrea finensis de Roissy, 5.243.2. - Patella finensis Lin. Gem. 3692. 3. Argenville I. A. 2. fo F. 


$$
C N C H O L E N A
$$

10. II. De ux a tu res à surface sillonée. Peut ếre des especes distinctes. L' ane des deux ('I I) paroît fossile.

12. *aly p't ré e verruque use, peu conique, très aplatie, peàite, jaunâtre, transparente, garnie de points élevés rudes Calyptrea verrucosa mihi, Une calyptrée très delicate et très rare. Point d'auteur $n^{\prime}$ en a fait mention. 1'ignere son lieu natal. Elle a 5 lignes de diamètre et le sommet ne s'éleve point d'une ligne.

Concholepas. Concbolepas I. a m.

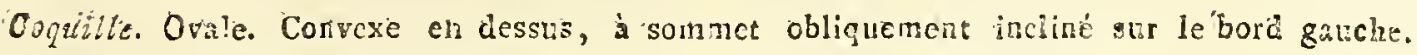

Deux dents et un finus à la base du bord droit.

Animal. Gastéropode ..... Portant un opercule corné.

2. Concholiépas péruvien, ovale, ventrae, trés baillanre; bord droit crenelé, échancré et bidenté à sa base; bord gauche contigu, bordé et reflechi; sommet de la spire obtus et caché.

"Concholcpas peru"viana Lam. F. "7o. de Roissy. 5. 245.

Buccinum concholepas Bruguière Ellcyclop. meth. vers. to 3. kp. 252. Argenaile, I. \&. 4. E. I.:.

DEU IEME SOUS. DIVIS IO

Cogulle anivalve anilowiare, spirivalue, engainant ou contenant banimal.

A. Ouverture échancrée ou canaliculée a s a b ase.

Côn e. Contss. I i n né.

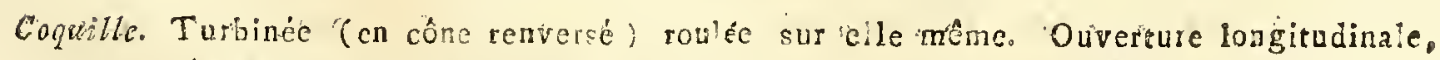
étroite non dentée, versante à sa base。 
Animal. Gastéropode à tête munie de deux tentacules qui porlent los yeux près de leur pointe. Manteau étroit. Un tube au dessus de la tête pour la respiration. Le pied muni d'un opercule petit, elliptique, corné ou cartilagineux.

I. Cônes à spire couronnée de tubercules.

1. Cône cedonulli, conique fasciée par des taches separées ou réunies; marqué de plusieurs lignes circulaires pointuées, la spire concave aigüe. Conus cedonulli Bosc 5. I I4. de Roissy, 5. 404. I. Conus ammiralis cedo nulli I.in. Gm. 3390. 10. $\delta$. Une variéte qui vient de la Chine ou de $C_{z-}$ racao, et qui a beaucoup d'analogie avec la figure de $\mathbb{K}$ morr Del. VI. I. 13. f. 5; mais le dessein de notre cône ef́t plua regulier.

2. A m i r a l, brune jaunâtre, à taches blanches triangulaires, et à bandes jaunes finement réticulées; fpire concave aigüe Conus a m iralis Iin. Gm. 3378 n. IO. de Roissy. 5. 406.4 .

Exemplaire de belle grandeur, et avec la clavicule bien élevée.

3. A miral, variété à cordons réticulés fur la bande blanche du milieu; celle de l'extrémité n'en a point.

Grand amiral vulgairement.

4. Grand a miral, autre variété à deux cordons réticulés fur la bande blanche du milieu; et à un feul fur celle de l'extrémité inférieure.

5. Le $\mathrm{Vice}$ a miral, la bande du milieu fans cordon

ore fans bandelette.

6. L' a miral d'or ange, rose, à deux larges zones orangées, et à grand nombre de petites, tachetées de rcuge brun.

Conus a a as acus Lin. Gm. 3392. 56. Martini 2. t. 57. f. 636. 637. p. 282 .

C'est le cône le plus rare et le plus précieux, des Indes. Notre exemplaire a deux puuces deux lignes de longuear. 
6. b. l'Amiral de Runiphins, brune, ou orange à deux bandes composées de taches triangulaires blanches.

7. 8. C. P iqu re de mo u che, conique presque ovöide bianc parsemé de piqures noires, disposées en masses, la ipire obsuse; moucronéc ou aiguë la base echancrée.

Conus arenatus Bosc. 5. I I9. C. ftergus muscarum Lin. Gm. 3395. 23. Martini 2. t. 63. f. 696. 697.

9. V a ri ét é à piqures plus rappochées.

10. Varićté de la même à piqures plus claires et à tubercules de la fpire plus élevés.

II. V a ri ét é à tubercules plus rapprochés et à piqures réunies à différens endroits en zigzag.

12. V a r i été à base presque tronquée, à clavicules pas plus élevés que les tubercules.

13. Variété de la même à piqures regulières.

I4. V a r i é $t$ è de la même à côtes élevées granuleuses.

15. 16. Variété de la mềme à gros points bruns le ftercus pulicis de Rumph et des hollandois; V. Martini. 2. t. 63. f. 698. - Bosc 5. II 9 . en fait une espèce diftincte, Conzs pulicarius, et á ce que je crois, avec raison.

I7. Cône papier marbré, conique rarement granuleus, brun ou jaune marbré de brun ou de blanc; la fpire aigüe. Conus nebulosus, Iin. Gmel. 3397. n. 29. Bosc. 5. IJ 5. Martini. 2. t. 62. f. 683 .

18. Cone nocturne, conique, noirâtre, marqué de taches blanches cordées, jointes entr'elles et disposées en fascies; la fpire obtuse; la base granuleuse.

Con us nocturnus Bosc. 5. 116. Martini. 2. tab. 62. fig. 697. 688. Chef d'Escadre, Contre-Aniral, vulgairement. - des Molragues.

18. b. c. Deux variétés plús petites à couleur brune plus pâles et à taches blanches plus petites ou plus grandes. 
19. 20. Ise da miar. Oblong; rarementgranteur; noir; marqué de tactes blanches, presque triangulaires, la tpire obtuse, canaliculée.

Con us ma.1 m.oreus. Lin. Gmel. 3374. 1. Bosc. 5. 115: Martini, 2. t: 62. f: 685: 686. - de 1'ocean Asiatiqute.

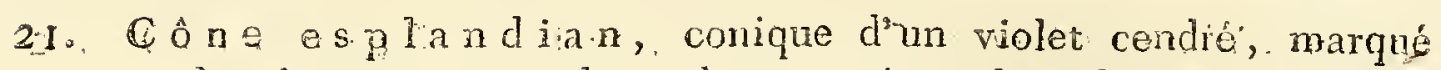
de plusieurs rangs de taches carrées, blanches et; de lignes. parallèles, couleur de foffrans: la fpire obtuse, caralieulée. mucronée.

Qonus arachnoideus, Lin. Gmel. 3388.34 -.. Martini 2: 6I. fig. 076. des Molugues; vulgairement: to the đíAraignée.

22. 23. CÁne imp e.ria l", conique, blanchâtre, gani de fascies alivâtre et de lignes transverses, artiçulées de blanc et. de: brun; la fpire obtuse, comprimée ou élevée.

Conns imperian is Lin: Gm: 33.74. 2. Mose. 5\% 1:1\%: Martini 2. tab. 62.f: 600. vulgairement, Couroune impés. riale, Konculorn; - des Moluques. Lune a des, lignes. tanyerces bien largos et pates, l'autre plus ctroites et plus. foncées.

24. C Q̂ $n \in \mathrm{m}$ a $\mathrm{u} \mathrm{r}$, conique, noirâtre, tachẹté de blanc, garni de lignes transwerses interompues noires; la fpire obtuse. Con as fruscatus Bosc... 5. I 18. Martini. 10. to 139. I289. - dans l'océan Indien et Afficainl.

35. 26. * Côn e r are, conique, brun", femé de petítes rugosités: blanches d'émail, la. fpire obtuse moucronnée.

J. ignore fon lieu"natal:. L'un eft plus petit et a des. cor-dons bruns,foncé plus granulenx et plus élèvés.

127. Ife fpectre, conique, bran, tacheté de blanc. Conazos Spectrum Lin. Gm. 3395: 62.

Une rariété rare i taches blanches presque quarrées. 


$$
\text { C. Oे: N E S }
$$

28. 29. Cồn e p a p̉er t u r c, conique, glauque, tachetéde fauve: marqué de lignes: transverses articulées de blans et de-brun, Jit fpire obtuse.

30. 3I. Cône hebräque, conique, blanc quelque foĭs rosé, marqué dè taches eransverses, presque carrées, noires, la spire convexe.

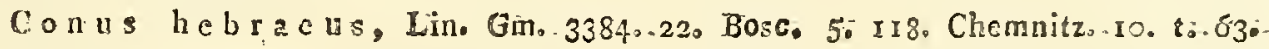
f. 699. 700.

32. 33. Còne he bra î u e d'un beau volume; lè vrai. cône dè ce now àe Linnée et de II a.rti.ni, 2. p. 259. T. 56. 16.17 :

34 Cône fustigé, conique Blanc, marqué de gouttes noires inégàles; la spire comprimée moucronée, la base échancrée...

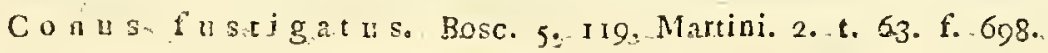

35. 36. Cône pea u de chagr.in, conique muriqueax ous granuleux, blanc tacheté le plus souvent d'orange, ou de marron, la spire aiguë.

Conus vari us.tia. Mus. Lud. VIr. 5590 n. 269. L. G. 3395.240 -

Busc. 5.- 120 . Marrini 10. to 238. f. 1284.

37. 38. Cône peau de chagrin, belle variété orangé; avee: des taches blanches plus ou moins grandes.

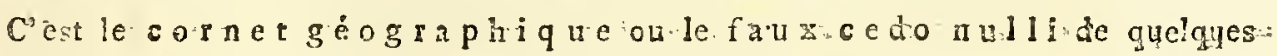
autcurs. Martinine. to 61. f. 678, 679:

V. une milleur figure chcz Regenfu fs.rvIF g.. 10 .

39. 40. De $u x$ varié $t$ é $s$ du mème cône à taches marrons; lé piús petit présente des-taches alongées non interrompres:

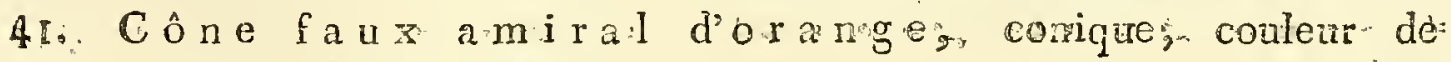
rose; fascié de brur.

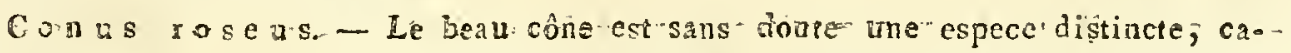
raciériśe par ses côtes iransverses élevées.s De bonnes figures s'en trouvent:

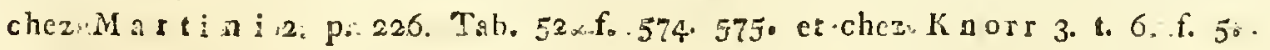

49. Cổn e bu is já un a, conique; jaune; spires noueuses et à: stries: grenues.. 
Conus citrin us mihi. - Der getbe Steinalmiral, die kleine gekrönte Zitrontute Martini, 2. p. 328. t. 61. f. 681. de Couracao.

43. Côn e rustiqu e, conique, brune, à spire couronnée à bande blanchâtre.

Conus rusticus; Lia. Mus. Lud. Mr. 556. n. I63. Gm. 3383.18. 44-46. Trois variétés à couleur brune pâle ct tachetée de blanc. 47. Var i été brune-foncé à fascie plus mince; elle paroit même différente comme espèce.

4. 49. De ux cồnes, à spire couronnẻe, depouillés. I’une a beaucoup de ressemblance avec le combs candidus de Born Test。 Tab. VII.

* Cônes, á rpire lisse et en partie aplatie.

50. 51. Cóne émail, conique, blanc d'émail, fans la moindre taches a robe femée de petites rugosités, la fpire plane, avec une tres petite pointe.

Conus porcellaneus, testa conica, tota alba, longitudinaliter rugosa.

Ce cone eft trés rare s̀ cause du defă absolu de couleurs, qui ne peuvent pas être ôtées par Part, vu que lés rugosités longitudinales rendent le depouiliement impossible fans applanir ces rayons élevés, diftans, et irrégulierement placés. Cette espece a tellement l'apparcnee de l'émail ou de la porcelaine, que je ne voulois point croire à mes yeux fans le fecours de l'acide nitriquc qui Y produifoit la mêtne cffervescence que sur les autres. La patrie en"eft inconnue.

52 - 54. Cone tigre, Blanc, marqué de fascies jaunes et de plusienrs rangs de taches brunes; la fpire plane, obtuse.

Cous liticratus, Lin. Gm. 3325. 3. Bosc. 5. 125. Martini. 2, tab. 6e. ث. 668: Trois variétés provenant du deponillement; - de l'océan Asiatigue.

55 - 60. Six tigres à bandes, du même endroit.

61. Cone tỉgre, variété à bandes jaunes.

Der Pardermit gelben Banden, Martini. 2. p. 3r2. Tab. 60. f. 670 .

Regenfuss. Tab. iV. f. 46 .

62 - 64. Cône pavé, blanc, marqué de fascies jaunes, peu apparentes, et de taches transverses fauves; la fpire obtuse; la base fillonnée. 
Conus ebu:nẹs, Bosc. 5. 125. Martidi, 2. tab. 6r. f. 674. - dans la mer des Indes.

65. La même fillonnée longitudinalement.

66 - 68. Cốn e mosa ì u e, blanc, marqué de plusieurs rangs de taches écarlates ou de taches informes, couleur minime; la fpire plane, obtase, la base fillonnée, violette dans l'intérieur.

Conus teffellatus Bolc. 5. 125. Martini 2. tab. 59. f. 653. 654. vulgairement nattes d'Italie. - Se trouvent dans la mer des Indes. La conleur eft très diffsrenle dans les trois individus de mêrne que les taches; le taches fone plus ou moins cohérentes danś l'uue, diftantes daus les autres.

69. Cône flamboyant, brun ou orangé, marqué de fascies blanches interrompues, ou blanc taché de brun, la fpire applatie, trés élevée au centre, la base noire.

Conus gencralı Lin. Gm. 3375. 4. Martini. 2. p. 29r. tab. 58. f. óf9 et fa.

70. Variét é à prémier cordon plus large.

7r. Variété de la mêne à bandes oranges très larges.

$72-74$ Trois variétés de la même à bandes plus ou moins foncées, plus ou moins distantes.

75. Côn e fileur, blanc, marqué de taches longitudinales brunes et de fils nombreux transverses interrompus; la spire obtuse,

Conus lineatus Bosc. 5. 12\%. Chemnitz. 10. to :38. f. 1285.

76. Cône renard, fauve; la spire canaliculée, plane, obłuse, la base granuleuse; le fond de l'ouverture violet.

Conus vulpinus Bosc. 5. I27. Born Misus. caes, tab. 7. f. I3. le vôtre eist. depouillé

77. Cốne rou il l.é, couleur de roullle, marqué d"une faskie b'anche, et de fils fauves peu apprens: la spire obtuse.

Conus Senator Lin. XII. I168. n. 300. Gin. p. 3351. 12:

Conus ferxugiacus Bosc. 5. 128. Martini. 2. t. 59. 5.659. 
78. Cone cierge, jaune: Ta spire plane, obtuse, la base violetre.

Conus virgo Bosc. 5. T28. conus cercola Trantini 2. p. 234." "4. 53. f. 586.

79. Cône carotíc, Rouge ou 'jauné, tacté ou fascié de blanc; la fpire plane, obtuse; le fommet couleur de rose.

Conus da.ucus. Bosc. 5. 128. Conus arausiacue Martini et Chemnizz X. p. 92. T. 144. A. fo 1. vulgaircment: Carotte rouge.

So. Cô ne carsot.te, individu très petit et d"une couleur plus rouge.

81. Cổne capittaine, Jaune rerdâtre, marqué de deux fascies blanches, tachés de brun; la fpire légèrement convexe. Conus capitane us Lin. Gm. 3376. 6. Bosc. 5. I28. Martinï 2. 1. 59. f. -650 - 652. de l:0cían asiatique.

82. 83. Cône hermine, Verdâtre, marqué de deus fascies bianches; la fascie fupérieure variée de noir, 'I'inferienre nucompagnée de deux rangs de taches noirâtres.; la fpire plane, obtuse.

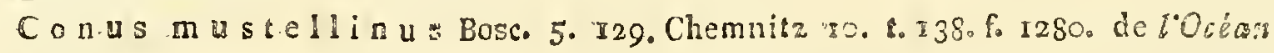
asiatique.

84. Cổ ne lo u p; Blanchâtre marqué de lignes brunes rameuses, réuniees, la fpire obtuse.

C.onus fumatrensis Bosc. 50 129. Chemnitz, 10. to I4t. f. a. b. Princegs fumatroe.

85. 86. D.e nx variététs, qui présentent les raies longitudinales fauves foncées et les bandes plus jaunes.

87. Cóne aile de papillon, Rubicond, marqué de plusieurs zones inégales, articulés de brun et de blanc; fpire plane, coveze, mucronée.

Conus genuanas Jin. Gm. 33381. 14. Bosc. 5. 180 . Martini 2. t. 56. f. 624. Un individu de belle grandcur, des côtcs de l'Afrique.

88. Cône aile de papillon, Exemplaile qulus petit mais à couleurs très vives. Les cordons de la base font très élevés. 


\section{C $\hat{O} N E S$.}

89. Cône papilionacé, blanc zoné de taches et de points fauves, la spire convexe, légèrement canaliculée, mucronéc.

Conus papilionaceus, Bosc. 5. 13r. P1. 40. f. 4. Martini 2. 6. 60. f. 669 .

90. Cône papilionacé, d'une granden extraordinaire. Il a 3 pouces 3 lignes de long, sur 2 ponces de diamétre à la spire, qui est très aplatie.

9I. Cône glauque, glauque, marqué de lignes transverses, faures, interrompaes; la spire convexe, tachée de brun; la base échancrée, striée; le bord interne de la lèvre droite, couleur de rouille.

Conus glaucus Lill. Gm. 3392. 15. Bose. 50 I32. Chemnitzo ro. t. Ias. fig. 1277. 1278. Vulgaireiant, Pelotte de berare, Tinne de beurre, Chat. de Cliypre.

92. Variété à lignes transverses ponctuées, à bande large glauque.

93. Variété plns petite glauque à points formant les lignes transverses élevées.

Conus millies punctatus Chemuitzo ro. po 460 t. 540 . f. r305. Seba. Tom. 3. to 43. f. 36. 37 .

94. Cône moine, ovale, ondé de brun et de blanc cendré, quelquefois marqué de zones articulées; la spire aigue; la base sillonnée.

Conus Manachus, Lino Gmo 3382. 16. Bosc. 50 ₹320 Regenfufs, Io t. 52. f. 68. Knorr. Tom. 3. f. 2 3. dans l'océan oriental.

95. Exemplaire dépouillé en partie.

96. Cône mo ine, d'une grandeur considérable; il a quatre poucés de long et píus de deux de diamétre.

97. 98. C o $z_{1}$ e ress a u, ovale blanc ou jaunâtre, marqué de fascies treillisées de jaune ou de fauve; Ia spire conveze. Conus mercator, Lin. Gm. 3383. ro. Eosc. 5. I33. Adarsna P. 6. f. 3. Martioi. 2. t 56 f. 620 . 621. - Sur les côres orcidertales de l'Afrique.

99. Cône tinné, citrin, marqué de plusieurs rangs de taches brunes, la fpire convexe, mucronée, tachée de brun; la base échancrée, ridée. 
Con us berulinus, Lin. Gm. 3383. 2c. Bosc. 5. 132. Marrini 2. t. 40. f* 665. p. 308. der gelbe Tiger, Tinne de beurie, vulgairement; un fuperbe exemplaise des Grandes Irides.

100. I01. Côn e $\mathrm{m}$ in i in e, couleur de rouille, marqué de files transverses très nombreuses, couleur d'ocre; la fpire con vexe mucronée; la base échancrée.

Conus figulinus. Lin. Gm. 3384. 21. Bosc. 5. I34: Martini. 2. t. 56. f. 656. Vulgairement: la Minime, la fileuse Dav. Thread-ftamper Peniv. braune Eichenholakegel; - deux individus de belle grandeur, l'un avecunelegère fascie jaunâtre; - des Indes.

I02. Variété du cốne $m$ in in e de belle grandeur à lig= nes transversales interrompues.

C'est conus minimus Lin. X. p. 7I5. n. 267. tefta cinerascente (le nôtre ct rougeâtre) punctis oblongis cincta. L'échancrure de la base eft la nểe. Le cóne me paroit une variété dépouillée. Il faut rayer dans l'édition de Linné par Gmelin, les figures citées pour le Minime p. 3382. n. I7. ainsi que celles qui d'après lui forment des variérés du figulinzus $\beta$ et $\gamma$ et la désoription du ninimus eft à fubordoner à celle de figulinus comme variété pour la quelle nous n'arons pas de figure.

103. Côn e I i n é, Jauñe, marqué de lignes transverses très nombreuses, couleur d'ocre; la fpire plane, obtuse, "(mucronée) la base róboteuse?

Conus que r cinus Bosc. 5. 108. Martini. 2. t. 59. f. 657. Knorr. 3. II, 2. - des Indes.

104. Variété du même à couleur jaune très pâle, et à fascie blanchâtre.

105. Cốn e protée; blanc marqué de taches et de points bruns disposés fur des lignes régulières, la fpire obtuse, mucronée. Conus profeus Bosc. 5. 13ł. Martini 2-t. 56 f. 627. vulgairement, ffectre, gueule delìon, lion combatíant; - ce cône n'ct pas commun et fe trouve dans l'océcun Américain et Indien.

106. Variété yare du même à taches en zigzag feparées par un cordon, formé par des points. C he m $\mathrm{i} t z 10$ t. 140 . E. 1300 . 
IOY. C今n e leonin, Blanc, marqué de taches longirudinales, irrégulières, brunes, et de gouttes brunes disposées fur plu. sieurs rangs; la fpire plane, canaliculée, mucronée.

Conus 1 eoninus Bosc. 5. 135. Martini 2. t. 57. f. 640. Tom. 10. P. 446. f. 2099.- - vulgairement: der vestindische Aclmiral, der Klimmencb Löwe leo scantens, fauss aniral de Guinée; - nôtre Individu est de Gurnzée th de belle grandeur, deux pouces quatre lignes de long.

$107^{b}$. V a riété jaune du même cône.

108. Cône picoté, Jaunâtre, fascié de brun foncé, marqué de plusieurs points obscures infiniment nombreux, la spire obtuse.

Cou us a ugur Bosc. 5. 135. Martini 2. to 58. fo 64i. de l'océan Asiatique.

109. Côn e a madis, brun, orangé, parsemé de taches blanche cordées, presque triangulaires et réunies; la spire canaliculées, le sommet saillant?

Conus amadis, Lin. Gm. 3338. 32. Mattini.2. to 58. f. 642. 643: - de l'océan Indien.

IIO. II I. Deux a $\mathrm{m}$ a dis à taches blanches plus grandes. Chennitz. IO. t. I 42. f. I 322. I 323.

I12. A madis, avec une excroissance singulière, qui provient d'une plie de la lèvre.

113. II 4. Cổ ne caractéristique, conique, blanc, avec des taches brunes en forme de caractères placés en trois séries, spire aplatie, mucronée.

Conus caracteristicus, tefta conica, alba, characteribus sufescenti. bus in triplici fascia inscriptis, fpifa truncata obtusifima, alba, ex fusco maculata, basi ftriis exoratis cincta. Chemniezo 10. p. 54. to 182. f. 1760. 176 เ.

II5. Variét é du même à taches confluantes.

II6. Variét é rare á fpire tout à fait tronquée, à fascies interme diaires blanches non ponctuées.

II ๆ. II8. Cône vario lé, fillonné, granulcux, blanc oú jaunâtre, varié de fauve; la fpire élevée.

G o nus versucoses Bosc. 5. 24. Martini. 2. 1. 55. f. 6r2. C. 
I19. Cóne noble, Jaune de citrorr, marquẻ de taches blanches ${ }_{x}$ cordées de deux fascies composées de lignes ponctuées distinctes; la fpire plane, concave accompagnée $d^{2}$ un rehord. aigu; le fommet raucroné, couleur de rose; la base échancrée.

Conus no bilis Lin. XII. p. 3r68. ก. 303. Gm. 398\%. T3: Chemnitz. 10.

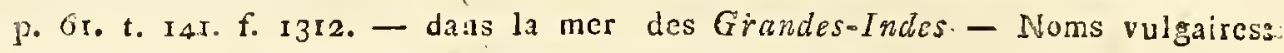
Ic Damier chinois; bella Donna, gecle Tygers Tour.

120. I $2 \mathrm{I}$. De ux vari ét és très. rares à couleurs plus pâles, et taches plus nombreuses.

I22. Cône Thomas, orangé ou blanc marqué de zones et de lignes nombreuses ponctuées, ld spire concave, macronée. Conus Thomae Lin. Gmel. 3344. 70. Martiuj. 2. Fo. 237. Tab. 53. f.590. Bosc. 5. 14.4. - vülgaircment par corruption. Cóne dioma.

123: I24. * Cône gé a $n t$, jaune citron avec des: taches ế trois: zones blanches cordées et ponctuées de jaune, la spire plane, sillonées mucronée, flamboyante de brun et de blanc. Conus gigas, testa laevi flava albo-maculita, zonis, tribus albis, punctis favis diftäntibus. cinctis, spira plana, sulcata, mucronata.

C'est parmi les cônes cleui qui reçoit le plus de vo lume; à conclure an moins d'après les exemplaires que $j$ 'a devant moi. Is un a 5 pouces 10 lignes de longueur, et 3 pouces 4 lignes de diamétre. Foint d'auteur n'en a fait mention: Lister à fait représenter Tab. $77 \mathrm{I}$ un cồne de la même grandeur, sans $\mathrm{y}$ ajouter une phrase caractéristique, nais le sien appartient aux cônes cylindriques, à spire élevées; le nôtro est conique à spire applatie, dont seulement les deus 'dernier's contours qui forment le sommet sont un peu plus élevés;

$J^{\prime}$ ignore fon lieu natal.

325. Côn e g é a n t, exemplaîre plus petit mais à conleurs plus regulièrement distribaés. 
* * Cônes à s pirenon couron ée, a longég e t subcylindriqu:es. vulgairement: roul eaux. ou cylindres.

126. Cône bru.nette, oblong, strió transversalement, brua, marqué de taches inégales, triangulaires; blanches; Ia spire aiguë.

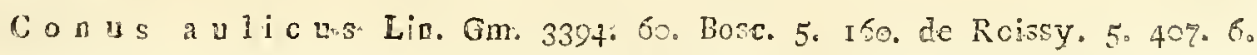
Martini. 2. t. 53. f. 592. - de la mer des Indes. - Vulgairement: Brinetios Drap'd'orange, Netakorn, Silberstück: - Notre individu esi de belle grano deur, de 5 pouces de longueur, cr d'un brun - foncé.

I27- - I29. Trois brunettes plus petites, bunes-foncé, à taches blanches plus frequentes, l’ùne comme parsemée avec dê petites taches.

I30. Brunette, couleur brune dè girofle..

131. Bru nette, couleur d'orange:

332. I33. Doux but ut tes coulew dorange à taches blanches plus petites et plus fréquentes.

Bosc paroir faire des cônes oranges une espece diffincte fous le nou de àr a g orangé, Conus a uratus go 150 . Martiai 2. t. 5ł. f. 597. mais śśne se diftingucat des brunetres que par la coulcur orange.

134. C’ôr e drap d'ôr, Jaune', marqué de lignes longituảinalús onduleuses brunes et de taches cordées, branches, circonscrites de fauve; la fpire élovée.

Conus.textile Lib: Nil. p. II7? 1: 359. Gmel. 3393. 59. Bosc. 5. $153^{\circ}$

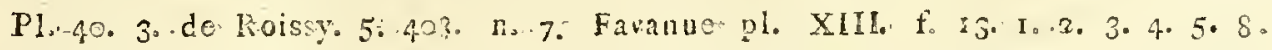
C. Io.Martiri. 2. t. 54. f. 54.8.599. Gc0.-Vulgairement: drap d'or, drap it reseaul, das braute. Feld-oder Hajel huhn, das Kläppeikitssen, die fpilzo berger - des mess entre les Tropiques. Individu magnifique d’une grändeús extraordiana, 4 pouces de longucur.

335. Cồne dirap dor, plús petit et ầ couléurs moins vives.. -

136. Cône drap d’or, variété à lignes löngitudunales plus onduleuses presque en zigzag.

137. 138. Cône drap d'or, deux variétés àtaches blanches per. fréquentes. 


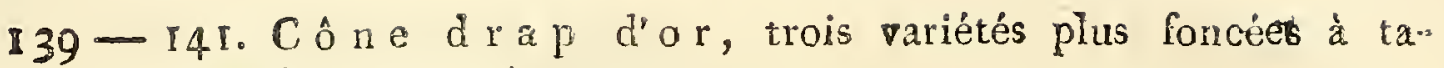
ches blanches très petites.

I42. - 145. Cốn es dr a p d’or, quatre variétés â couleur juune passant à la brune, à zones plus minces et à taclies blan=ches plus frequentes.

146. I 47. Cônes drap d'or, deux variétés rares à couleur oranges presque uniformes à lignes longitudinales ondulées très rapprochées et parallèles.

Il faudra faire de ces deux derniers cônes une nouvelle espiece.

148. Cône ftrié, brun avec des taches blanches on rougeâtres; de fines ftries transversas parallèles, brunes diftinctes; la fpire obtuse, canaliculée; la base fillonnée.

Con us ftriat us. Lin. XII. p. II7I. n. 318. Gmel. 3393. n. 53. Bosc. 5. 146. Adanson pl. 6. f. 2. Martini. 2. p. 35I. Tah. 64. f. 714 - 716. Vulgaio rement: l'Ecorchée, das Wulkhorn, der gestreifite Tiger; - des mers de l'Afrique Orientale er des Indes.

$149-153$. Cin q $\mathrm{n}$ u a n c es de la. couleur brune et des taches rouges de chaile.

154. - I56. Ciône ftrié, trois variétés très belles, oranges, avec les mêmes taches couleur de chair.

557. I 58. Cô ni e tuli p e, oblong, rougeâtre, varié d'ondulations blanches et bleues, marqué de lignes nombreuses circulaires, brunes ponctuées de blanc; la spire canaliculée.

Conus tu Iipa Liñ Gin. 3395. 64. Bosc. 5. r20. Mntini 2. t. 64. f. 718 , 719. vulgairement: la tulipe, le taffetat, la geogrophique, die gewölkte Achattute, la Nuée; - il se trouve dans les mers entre les Tropiques.

159. I60. Cón e ne bu $\mathrm{l}$ e ux, blanc, fascié par des taches longitudinales, nébuleuses, brunes; marqué de lignes transverses, ponctuées de blanc, la spire obtuse, presque mucronée.

Conus magus Bosc. 5. I46. Martini 2. t. 52. f. 570.583, vulgairement: da sebuleuse, dic kleine geuoolite Achattute; - de da mer des Iriles. 
I6I- - I64. Cone nebule u $\mathrm{x}$, quatre individus d'une variété à fond bleuâtre et à deux bândes jauıes. Les lignes ponctuées de la base sont élevées.

$165-\mathrm{I} 67$. Cône. $\mathrm{n}$ ○ buleux, variété olivâtre à stries moins sensibles; trois individus différens.

168. Cône solide, nuagé de brum et de blans transversalement strié, des stries nombreuses ponctuées de blanc et de brun, la spire élevée subcaliculée.

Conus solidus, testa, conica crassa, transversim striata, ex fusco et albo nubeculata, tilis seu seriebus numerosissimis ex albo er fusco punctaris, fascia lata a'ba redimica, spira pyramidata (sub) canaliculata. Chemnitz. Io. p. 59.

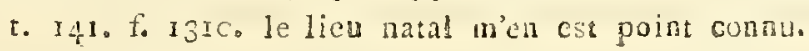

169. Ciône poudingue, brun, marqué de taches cordées inégales, blanches, la spire obtuse.

Conus rubiginosus, Bosc. 5. 15t. Matini 2. 4. 54. f. 594. 595. de l'océan Asiatique.

$\$ 70-172$. V a r ét é du même dans la nuance de la couleur, et dans la disposition des taches; trois individus differens l'un de l'autre.

I73. I74. * Cóne àilé, brun, marqué de taches blanches en forme d'ailes, disposées fur deux bandes paralèles, la fpire élevée, canaliculée.

Conus alatas, testa conica fusca, maculis albis alaeformibus, feriebus binis insignita, fpira canaliculata pyramidata.

Cette belle coquille dont je ne connois pas la patrie $n^{2}$ a pas été décrite par aucur auteur. Flle a I pouce 9 lignes de longueur fur 1 pouce de largeur.

175. 176. Côn e nussate 11 e, oblong, ftrié transversalement, blanc, nué de fauve, ceint de lignes ponctuées brunes; la fpire convexe élevée.

Conus nussate 11 a Lin. Gm 339). 43, Bosc. 5. 159. Martini. 2. t. 5x f. 567. Kinoss. 3. p. 4.0. 4. 19. f. 4. - de da res des Indes. Vulgairement. 


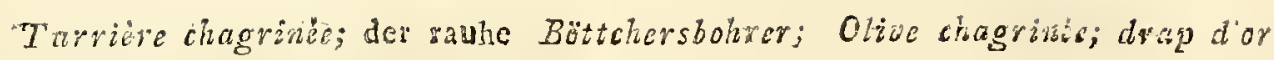
piqueté de la Chine,

17\%. Cône nuss a c l l e d'un beau volume à points plús grands. I78. I79. Cône târière, Rougeâtre, marqué de stries élevées et de deax fascies d'un jaune pâle; la pipire pyramidale, la base violette.

Conus terebelier Lir. Gm. 3390. 44. Co terebra Bose. 50 145. Chemitro

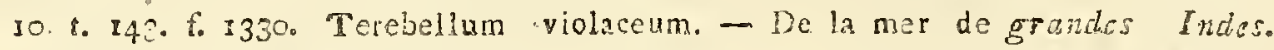
L'u de ces.denz individus eft bien remarquable en ce que ies firics font grao nulcuses de forte que les lignes forment conane aunat de chapeiets rapprochís.

179. 180. * Cône f'usiforme, rouge-rose arec des stries élevées et des fascies blanches, la fpire dbruse.

C o.n as fusifornis, telta fubconica, transversim ftriata fasciis duábus albis.

Ce cône doit former une espece nouvelle qui fe distinque - des autres cônes tarrières par le corpss peu veritru, par la ipire plus obtuse que dans les autres et par fa grandeur. I' un des deux individus que nous possedons, a deux pouces cinq lignes de lengucur, fur I pouce de largeur. J'ignore fon lieu natal. Je I'ai reçu fous le nom de bout de chandelle.

* * * Cônes cylindracés à fpire couronnée.

I8I. Cône broca rd, Oblong, marqué de nebulosités blinches et fauves et quelquefois d'un reseau blanc et brun; la ipire obìuse.

Conus geographus Lin. Gm. 3396. n. 65. Bosc. 5. 520. Martini. 2. to 64. f. 717. Favanne. 2. pl. 19. f. L. I. - des mers de l"Indes. Vulgairement: le brecard de Soie; tas.Wolkchen, die achatne Kronbacke; Wolkentute; Cloudy - fatuper.

$152 .-185 . \mathrm{B} \mathrm{r} \mathrm{ocards}$ de différente grandeur et couleur mais de la même espéce.

186. Broc a rd, belle et grande variété a deuri fascies transversales blanches. 
Porcel aine. Cyprace Lina a i.

Coq. oraje. convere, à bords roulés on dedans, Ouverurc longitudinale, étroite, densé Jes deux cotés.

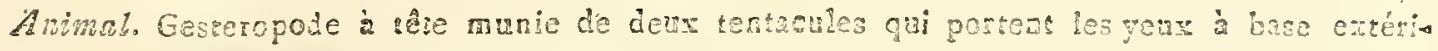
eur. Manzea formane deux grandes ailes que d'arimal replie a volonte fur le dos de la coquille, la recouvrant en egrier. Point d'opercule.

Cyaraea lin testa unvalvis, involuta, fubovata, obtura, laevis. Aperiara veringue cffusa, linearis, untrinque dentáta, lóngitudinalis.

* Porcelaines aigües, quiont une fpire visible.

1. Porcelaine exanthême, Presque turbinée, ferrugineuse, ou olivátre, parsemée de taches rondes et pâles; la ligne longindinale presque rameuse; ( - les dents toujours Brunes ).

Cyprae a exanthema, Lin。ed. XII. p. II 22. n. 325. ed. Gm. $339 \%$. I. Bosc. 5. 79. Martini I. p. 363. to 28. f. 289. to 29. f. 298. - 300. Vulgairement le faux argus; un individu de très belle granden:; clle a 5 pouces et gielques lignes de longueur fur 3 poupes de largeur; Se trouve dans l'ucéan Araéricuin et Atlantiqne.

9. 3. Porcelaine exanthème, deur exemplaires plus perits à taches blanches plus petites, et à fascies longitudinales plus discinçes.

4. 5. Porcelaine exanthène, deux exemplaires à taches blanches plus grandes.

6. Porcelaine exanthème, à taches blanches en partie oculées avec le fond brun.

7. 8. Porcelaine exanthème, "deur ezemplaires ferruzineur plus claires avec de irès petites taches blanches rappochées.

9. Porcelaine exanthème, variéce belle or grande, brunâtre, à taches blanches très petites ot très rapprochées.

Les trois derniers indigidu No. 7-9. paroifent former j'espece oculce, Cypraca ocellata de Bos c. 5. 34. Martini. I. 4. 26. f. 257. 258.

10. Porcelaine géographique, Presque turbinée, marquée de caractères; la ligne longitudinale rameuse. 
Cypraea ma p pa, Lin.XII. p. Ir73. n. 326. ed. Gm. 339\%. 11. 2. Martia x. p. 34ว. Tab.25. f. 24.5. 246. fig ire mallvaise. Vulgairement: la géographique, Porcelaine montagneuse, carte géographique, Mappe monde, caapschineke. - des ners dis Indes.

1. Porcelaine argus, presque turbinée, presque cylindriques, parseinée d'yeux, quatre taches brunes en dessous. Cypraca Argus, Lin. Gm. 3398. 4. Bosc. 5. 80. Mastini x. 20. 28. 285. 285. - se trouve daus les mers des Indes et Atlantique.

12. P. to $\mathrm{l}$ t $\mathrm{u}$, obtuse, presque cylindrique; les extrémités comprinées.

Cypraca te tudinaria Lin. ed. XII. porr79. n. 329. Mus. Reg. Uls. p. 567. n. 182. noscitar figura subcylindrica, fusco maculata, adspeisa tanquam farinae pulvere rariore. Bosc. 5. 80. une très honne figure f'eu trouve dans les delices de Knorr iV. 27. 2. vulgairement: Lapin; Levreau, Lióve. La Tortue ou Caret; Baftart-cap. de la mer des Indes.

3. Tortue, variétés à spire moins diftincte; I.in. ed. XII. 1. c. variat absque et cum spira antice manifefta.

14. 15. Porcela in e inc a r n a te, presque turbinée, pâte, avec des fascies incarnates; la bouche violette.

Cypraca carneola Lin. ed. Xll. rr74. n. 33r. Mus. rege Ulr. p. 568. n. 183. Bosc. 5. 8r. Martini. I. t. 28. f. 287. 288. Knorr. 6. 17. 4. Bosc. Test. VIII. 2. vulgairement : das fleisch-horn, der rothe fuchs, Taupe à bandes zousses, Caffé au lait. - De la mer des Indes.

16. 1 V V ariétè? à fascies ferrugineuses, à bouche brune-foncé. La spire est diftincte mais enfoncée. Knorr. a fait de la dernière une taupe, parcequ'elle est phus foncée. I. 27.2.3.

5. Porcelaine majet, presque turbinée, bossue, livide, tachetée de livide et de rouge de brigue, marginée des denx cotés; le dessous applati.

Cypraca stejcosaria, Lin. Gmel. 3399. 6. Adanson. pl. 5. f. r. Sihroeter Eiul. I. I. 8. f. 5. Basc. 5. 8I. t. 39. f. I. 2. 3. Born. Test. VIII. 1. Se trouve sưr la cốe de l'Afríque.

39. Porcelaine t a u e, turbinée, presque cylindrique, testacée avec des fascies pâles; le dessous épais, brun. 
Cy praca Talpa Lin. ed. XIl. p. II74. n. 333. cd Gmel. 3400. 9. Mastini. 1. . 27. f. 273. 274. se trouve dans la mer des Indzs. Vulgairement: da togso pe, der Maulwarf, der Moll, topo cero

20. Un e variété de la taupe ples jaunâtre.

21. Une vari été de la ta u p e plus olivâtre, et brunâtre des deux cotés; d'un très beau volume.

22. Porcelaine a méthyste, presque turbinée; les cótés bossus; le dos violet.

Cy prae a amethyszea, Lin. XII. p. Ir74. n。33. ed. Gm. 340I. n. 10.

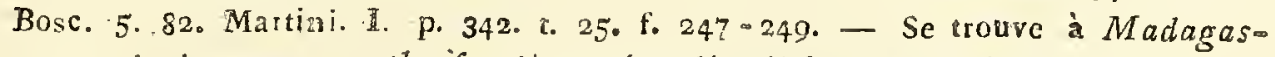
car; vulgairement : agathe fasciée ou banolée; lichte arabische Otter.

23. Variété de la même à cotés moins bossus et à dos plus tacheté de brunâtre.

24. Porcelain e pâle, Fresque turbinée, pâle, presque fasm ciée ; les extrémités jaunes; avec deux taches noires.

Cypraca I urida, Lill. Xl.1. p. II 75. n. 335. ed. Gmel. 340I II. Rosc. 5. 82. Martini. I. p. 384 . 20. fo $3 \overline{1} 5$. - fe csouve dans la mex méditerranée etc. - Vuigairemens: la Souris, de Mriso

25. La : $\mathrm{m}$ ê $\mathrm{m}$ e plus petìte à couleurs plus foncées.

26. Porcelai ne fragil e, Turbinée, ovale, verdâtre, ondée et presque fasciée de rowgeâtre.

Cy prace fragilis, Lin. Gm. 3402.n. 14. Bosc. 5. 83. de Born. Teft Vill. 6. - . Te trouve dans la mer méditerranée.

27. Tariété de la même à taches larges brunes.

28. V a riété à fascies blanches et à taches brunes petites et arrondies.

29. Porcelaine ce ndrée, Mince, ventrue, d'un cendré rougeâtre, avec des fascies plus pâles; l'ouverùre blanche. C y praea cinerea, Lill. Gm. 3402. I6. Bosc. 5. 83. Mastini. I. t. 25. f. 254. 255. On igrore fon pays natal. Notre individua quatre fascies et l'ouverture eft violetie pâle.

30. Parcela in e bistrion, ovale presque turbinée; livide, ocellée en dessous; plate, blanche; epaisse, noire et maculée de brun fur les cotés; la ligne du dos livide, l'ouververture violette.

$$
2^{*}
$$


Cy praea histrio. Lin. Gmel. 3403. n. I20. Bose. 5. 84. Chemnitz Conchyl. 10. Tb. 145. f. 834.6 . 1 847 . - le trouve dans la mer des Indes. Vulgaia renent: la vaic Arieguine. Cypraea Arlequina Chemniz ib. p. 110. Niñ excmplaire eft d'un beau volume.

3r. 32. Porcelaine a rabique, Presque turbinće, marquée de caracteres; la tache longitudinale firople.

Cypraca a a bica. Lill. Mus. Lud. 556. n. I8c, ed. Gnel. 33:8. 3, Rosco 5. 80. Krorr. 3. t. 120 f. 2. et. 6. ז. 20. t. 2. - fe trouve dans la mer des Indes. - Vulgairemene: la fausse Arlequine.

33. 34. Porcelaine zèbre, Turbinée, cendrée, avee des fascies brunes.

Cypraea zebra. Lin. Gm. 3400. 8. Bacc. 5. 8. Schroet. Einl. I. p. Ior. t. I. f. 6. - fe trouve dans la mer des Indes. Les nôties font d'un besux rolume, mais à robe plus foncte et a fascies plus claires.

35. Porcelaine ovale, ovale applatie, un des cotés bordé; le dos blanchâtre, avec des lignes ondulées très rapprochtées, d'un jaune brun.

Cy praca oyata Bosco 5.88 .

On ignore fon pays naral.

36. Porcelaine ocrée, oblongue, mince, blanche, pars semée de points fauve-rous ou junnès-d'ocre, fasciée do deus zones a points plus grands.

Cypraea Fertuginea, tcsta fpirali, elongata, tenui, alta panctis rubiginusis maculata et obscure fasciata. Maratini. 2. F. 344. t. 25. fo 250.25 s.

Vulgairement: das Eramerlingsey, Oeuf de Lorior; Kievitsohors ete. - de Madagascar.

3\% I. a même plus perite à taches plus alongées presque confluertes, variété a trois zones, formées par des points plus grands.

38: Porcelaine fanguinolente, mince, oblongue, fasciée de brun; les cotés ponctués de rouge.

Cypraea fanguinoleara, Lin. Gm. 3406. 38. Bosc. 5. 88. Martini, 3. 36. f. 265. 266. Oa ignore fon pays natal. 
39. Porcelaine àgouttes blanches, oblongue, mince jaunâtre avec des taches blanchos, les bords blancs à dents jaunes

Cypraca guttata, Lin. Gm. 33.02. 45. Martini. to to 25. f. 252. 253. Vulgairement: la neigense, die bechueite Porgellanes der gulbe Aigus rait wei"sen Augen, - Le lieu naral on eft inconnto

40. Va li é ćé te la même, plus loncée.

4 I Variété de la même plus claire.

42. I a mê me coupée au milíeu.

43. L a $\mathrm{m}$ ê $\mathrm{me}$, jeune individu.

44. Porcel a i n e olivâtre à taches ferrugineusés qui paroît être un jeune idividu de la porcelaine exantheme.

* Porcelaines obtuses, qui n'ont point de fpirevisible.

45. Porcela ine tète de rerpent; Triangulaire, bossue, postérieurement obtuse; le dessus maculé de blanc,

C y prac a Caput ferpentis, Lin. Gm. 3906. 39. Basc. 5. 89. Pl. 39. f. 4. 5. Martini I. t. 4九. f. 316 . - fe stouve dans la mes deś Indes et fus les côies d'Afrique.

46. 47. Porcelaine ma uriciane, triangulaire bossne; la partie poftériezre applatie, pointue; le desscus noir.

Cypraea mallitiana, Lin. Gm。 :40\%.4r. Bosc, 5. 90. Marini. r. t. 30. f. 3I7. 3Ig. - Dans la mer des Indes. Deux exemplaires de la premiere gia deuro

48. Porcelaine mauriciane tout à fait brune à zaches rares mais grandes.

49. Porcelaine ma n iciane, deponillée à 3 bandes on fascies; vola figure citée 319 de Martini.

50. Porcelaine tigre, ovale, postérieurement obtuse, antérieurement arrondie; la ligne tongitudnale tefacée.

C.ypraca eigris Lin. Gm. 34c8. 44. Bosc. 5.91. Martinai 3. 8. 24. R.232. -

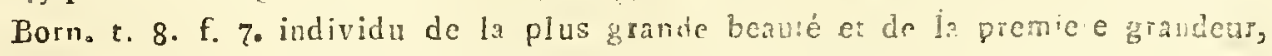
elle a 4 pouces 7 lignes de longueus sur 2 poures 2 hrgnes de largenro - Se trouve dans la mer des Indes. 
5I - 6ว. Dix tigres d'une belle grandeur présentant plusieurs différences de la grandeur et de larrangement des points.

61 - 64. Quatre tigres plus petices à points très fins et. très rapprochẻs. Les deux dernières présentent une différence singulière, en ce qu'elles font beacoup plus ventrues que les autres.

65. Porcelaine souris, obluse, bossue, cendrée, avec des fascies longitudinales brures, denticulées de noir.

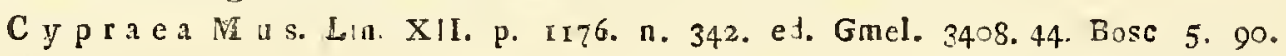
Martini. I. p. 324 t. 23. f. 222. 223. valgairement: la bossue de Carthageine; le Léopard; der katzenbauch; - se trouve dans la Mediterranée et sur les côtes de l'Amériquze.

66. 67. Porce l a $\mathrm{n}$ e souris, deux individus différens en grandeur et en couleurs plus foibles.

68. Porcela i ne 1 y $\mathrm{n} x$, ablongue, avec ides points bruns et des lignes faunâtres; le bout postérieur aigu; l'ouverture rousse.

C y praea Lynx, :in. "XIr. 1r76. n. 344. ed. Gmel. 3409. 48. Bose. 5. 92. Martini. a. p. 329. t. 23. f. 230 . 235. vulgairement: das kakerlakchen; der Luchs; la truitáe; - se trouve dans la mer des Indes.

69. Porcelaine ét o i lée, Mince, triangulaire, brune, à une bande dursale plus ou moins large formée par des taches blanches étoilées.

Cypraea ftellata, testa, fubtriangulari, etenui, crocea; dorso ex a'ba ftellato. - Porcellana in utroque latere fimbriata, dorso albido ficllulis fus is vario stc. Martini. 1. p. 39. t. 30. f. 326. 327. - Vulgairement: das fternclen, Ours in, ftarretje; - des Maldives.

7o. Porcelaine étoilée à bords moins fauves et à dos plus blanc.

* * Ombiliquées, à columelle perforée, et à forme cylindrique.

7I. Porcela i ne isabell e, d'un brun pâle avec des bandes blanchâtres, les extrémités jaunes. 


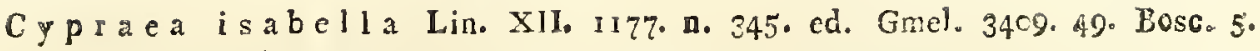
92. Martini. I. p. 357. t. 27. f. 275. - de la mer des Indes.

Notu. L i n a é et tous fes Comnentateurs ont placé celte percelaine parmi les porcelaines obtuses, mais quiconque les comparera dans la nature, la placcra cerrainement parmi les ombiliquées. Le mor de : fab-cylindrica doit aussi être ravé dans la descrifion fpécifique de Lin $n$ E. car prosure toutes les porce. landes ombiliquíes on cette forme; ce n'eft pas ainsi avec les obtuses, qui font pour la plus grande partic triangulaires govales, bossues.

72. Porcela ine isabelle ă couleurs plus pâles.

73. Porcelaine zígzag, des lignes en zigzag, jaunes rouges, et blanches; en dessous jaunes, ponctute de brun; les extrémités avec deux taches brunes.

C y praea zikzak, Linn. XII. II770 10. 349\% ed. Gmel. 3410. 54. Bosc. 5. 93. Favanne. pl. 29. f I. Martuni, Io to 23, fo $224-227$. - Vnigairement: le Zigzag, le point thangrie; - on ignue tou lieu natal.

74. 75. Porcela ine hirondelle, Blelâtre, les extrémités avec deux taches brunes.

C yprae a hirunde Lin. XII. 1178. n. 38 c. Gmel. 34Ir. n. 55. Besc. 5. 93. Born. V!II. II Marini. I. t. 28. f. 282. Vugarement: le petit ane bieuârue ou truité. Die fchwalbe. - Se rrouve dans la mer de. Irules.

76. - 78. Porcelaine aselle, Bhnche, avec trois fascies brunes.

Cy pratea AselItus, Lin。XII. I178. n. 351. Gmel 34ri. 56. Bosc. 5. 94. Knor r. IV. 25. 3. Martini. I. to 2\%. fo 280. 28I. Vuigarement: l'anon, te petit ane. - dans la mer des Indes.

79. 80. Forceldine a selle, à zones orangées.

81. Porcelaine atomataire, oblongue blanche, ponctuée de brun, les deux extrémités plus obscures avec deux taches.

C ур га а a a tomaria, Lin. Gmel. 3412. 67. Martini. I. to 28. f. 190. $29 \mathrm{~s}$. p. 37i. Bosc. 5. 96r. - On ignore son Iier natal.

82. Porcela ine zon a ir e, ovale, unie, jaunâtre, avec qquatre fascies courbes, tachetées de brun.

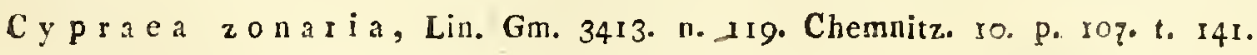
f. 1342. Bosc. 5. 99. - de la cồte de Guiaíe. 
* * * Porcelaines marginées ou qui or un renflement en leur bord ezériers.

83. Porcelaine crible, ombiliquée, jaune, avec des points blancs et ronds.

Cypraea cribraria, Linn. Xnt. II78. 17. 353. Gmel. 3819. 80. Bose. 5. 99. Martini. I. to 35. f. 336 . p. 403 . vn! gairement le petit argis ; das hleio are weibchen rom ergus; - se trouve dans la mer des Fódes ế la madjterranée.

84. Porcelain e crible à couleur jaune plus pâle, et à taches moins régulières.

85. Porcelaine monnoie, blanchatere, marginée par des nodosités.

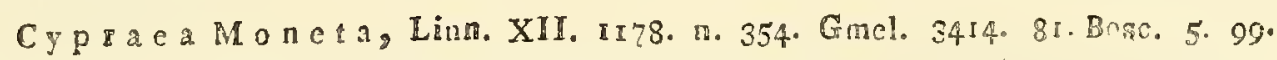
Marini, I. p. 404. t. 3r. f. 337. 338. vulgairemento Die colick, monnoie de Guinée, Cauris des Maldives, Squelette de Tortue, Slangerhoopipes, Sçhiangenköpfehen. - Se trouve dans la Méditerranée et la mer des Indes. Elle sere de monnoie on Afrique sous le nom de Cauris.

86. Porcelaine monnoic jaune.

87. Forcelaine anneau, le dos entowré $d^{3} u n$ cercle jaune.

C y p ra a a nnulus Linn. XIY. II79. In. 355. Gmel. 3455. 22. Bosc。 5. 99. Martini. I. p. 335. 1. 24. f. 239. 240. vulgairement: der gelbe Ring, puceiage blanc ou vio'et à cordon jonquille. - Se trouve dans la Medicerranée, et dans la mer des Indes.

88. Porcelaine caurique, le bord avec des gibbosités inégales, blanc, ponctué de brun; le dos d'un testacé nebuleux.

Cypraca caurica, Linn. XII. IIt9. n. 316. Gmel。 34.5. 83. Bosc. 5. I00. Martini. s. to 2\%. fo 30r. 302. 303. notse exemplaire f"accorde avec la figure 30 . mais les fries latérales brunes sont plus rares. Vulgairement: die Pockenporceliane, die shwarze Missem, Eiscnblattern, Rougeoie à grains moirs. - Se rouve dans la mer des Indes.

89. Porcelaine érosée, dentelée inégalement en ses bords, jaune, ponctuée de blanc, avec uno tache brune sur chaque coté. 
C, ур га а e rosa Linn. XI1. 3179. n. 357. Gmel. 3415. 84. Bose. 5. 100. Mnrtini. x. 1. 30. f. 320, 321. Vulgairement: Perdrix, Brandflecken, Kleine Leifte. - Se rouye dans la mer des Indes.

91. 92. Porcela i n e exosée, deux individus à taches plus rares et plus isolées.

93. * Porcelaine à pcints blancs, Jaunâtre, mince, parsemée de points blancs, dont quelquesuns font annelćs de brun, une ligne longitudinale blanchâtre.

Cypraea albo-punctata, mihi.

C'est la même coquille que Martini a figurée dans for ouvrage 1. p. 393. t. 30. 323. et décrite fous le nom de: weisse Friestlporcellane; quelques auteurs l'ont considérée comme une fimple variété de l'erosée, mais elle forme une espèce distincte, I. par les bords plus renflés et garni de traits bruns; 2. par sa forme plus alongée, 3. par son dessein; les taches annelées, $s^{3}$ il y en a, sont blanches et entourées de brun; une raie blanchâtre suit la direction de lá columelle et deus grandes taches violettes se trouvent des deux cotés.

94. Porcelaine à points blancs semblable à la précédente mais plus petite, et sans les raies brunes sur les renflemens des lèvres.

95. Porcelaine à points blancs, variété sans taches annelées. C'est la même que $\mathrm{M}$ a r t i n i a décrite sous le nom de la femelle de la rougeole ib. p. 3+9. f. $260.26 \mathrm{I}$.

96. Porcelaine à points blan $\mathrm{cs}$, variété singulière à raie longitudinale courbée en forme de lettre $S$.

97.* Porcela in e lu n u lée, alongée, jaunâtre, parsemée de taches blanches en forme de croissant.

C y p r a e a l u n a milsi, testa elongata, lutescente, maculis albis lunatis obducta.

Cette porcelaine qui par fa belle forme et fa beauté rivalise avec beaucoup d'autres, est nouvelle. Elle eft petite, d'urs 
pouce, deux lignes, de longueur, sur huit lignes de largeur, alongée, mirce, jaune foncé, garni de petites taches blanches en forme de demi lnnes. La ligne longitudinale qui separe le dos fait aussi distinction du desscin. L'autre moitié près de la lèvre renflée est blanche et garnie de points fauves. La lèvre elle - même porte des points imprimés bruns. Le des- sous est jaune pointillé de brun.

98. Porcelaine lunulée, variété à dos plus bombé, et à ligne longitudinale en zigzag.

99. Porcelaine folle, cendrée avec une tache carrée, testacée, à bords irréguliers.

Cypraea stolida Lin. XII. 1180. n. 360. Gmel. 3456. n. 89. Bosc. 5. sol. Martini. r. p. 37 r. t. e8. f. 292. 293. Vulgairement: la taupe, tête de Dragon, Dragonneau, Draakje; Born. VIII. Cypraea dracaena. - Se trouve dans la mer des Indes.

100. Porcela ine folle, individu plus petit, olivâtre, tache testacée plus regulière. Martini. I. t. 29. f. 305. Ces deux coquilles paroissent être diffèrentes comme espèces. Das Rostfleckchen, Porcellaine blanche à dos enrousillé de Martini. ib. p. 37 .

1QI. * Porcela in e ble ue, bleue, avec des taches testacée carrées, levre blanche sillonnée, violette en dedans.

C y práa ca e r u l ca, testa, clongata, tenui, caerulea, macul's rubiginosis adcpersa, intus violacea Martini. 1. p. 373. t. 28. f. 294. 295. Vulgairement : le bonnet polonois; der Blauricken; - on ignore son lieu natal.

102. Porcelaine pou, rougeâtre, sillonnée transversalement.

Cypraea pediculus Lin. XIr. Ir80. n. 364. Gm. 34r8. 93. Bosc. 5. 102. Martini. I. t. 29. f. 310. - Vulgaireisens: le pou de mer. - Se trouve dians toutes les mers.

103. Porcelaine pou, individu très petit à taches presque insensibles, Martini. 1. t. 29, f. 308. 309. 
104. 105. Por cela i ne à côtes, couleur de chair, pâle, le dos avec des stries élevées, très fines, et des aspéricés transversales.

Cyp rae a costata. Lin. Gmel. 34!8. n. 94. Bose. 50 102. Knorr. Del. 6. દ. I5. f. 7. - On ignore son lieu natal. Elle se distingú de la précédente, el ce qu'elle est plus bombée, que fa lèrre est moins ienflée, qu'clle est sans ta hes, et sons la ligne profonde longitudinale, qui interrompt les cútes transversàles de la porcelaine pow.

to6. Porcelaine noix, marginée des deur cốtés; lo dos tuberculé, les deux bouts saillans.

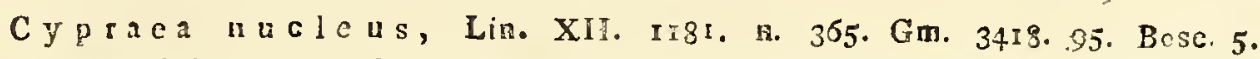
IC2. Martini, I. t. 2\% f. 3 I2. Born. Test. VIII. Vulgairement: la patite verale, Gransw oryzae, Porcelaine is grains de vis; die hasebianfs. - Se trouve dans la arcr des Indes.

10\%. 108. Porcela in e noix, deux individus plus petits, à robe plus foncée et à points élevés plus nombreux.

109. Porcela i ne 1 u e t te, saillante des deux cotés, avec des points élevés, sans stries; les extrémités jaunes; la ligne long tudinale latérale.

C y praea staphylaea. Lin. XII. Iy8r. n. 366. Gmel. 3419. n. 97. Born. Test. V1II. f. 17. Martini. 1. t. 29. f. 313. 314. Vulgairement: Porcellio, 1a Cloporte, der Kellerevtirm. - On ignore son lieu natal. - Norre individu est tout à fait blanc, sans taches jaunes.

110. Porcela in e 1 u e t te, d'un blanc-jaunâtre.

II 1. Porcelaine luette grise; à points élevés jaunes.

112. Porcelaine cicercule, saillante des deux côtés, et parsemée de points élevés, la ligne lomgitudinale presque centrale.

C y praea cicercula Linn。 XII. Ir8r, n. 367. Gm. 34rg. 98. Bose 5 . 103. Born. VIII. f. I\%. Martini. 1. t. 24. f. 243. 244. - Vulgairement: der korallenförmige Kneqf, das gelï̈rnte Knöpfcheñ. - Se trouve dans la mer des Indes.

113. Porcela ine globule, unie, alongée des deux cotés. 
Cypraea globulus, Linn。 Xir. Ir8r. n. 368. Gmel. 2419. 90. Bose. 5. ro3. Martini. r. t. 24. f. 242. Born. VIlI. f. 20. Vulgairenient: cas Ferichen. Se t:ouve dans la mer de l'lide. Notre individa, est jaune foncé avec des points bruns. Il y a quatre taches bruncs ell dessous, placécs presque en carré régulier.

$$
\text { Es peces fossilles. }
$$

14. Porcelaine gonflée, Cypraea inflata, ovata tumido gibbosa, laevis, obscure marginata. Lamarck. Annal. d. Mus. I. 399. 17. p. 225 . Pl. 4t. f. I. a. b. Lieu natal: Grignon en France.

\section{O $v$ w e. Ovula I a m.}

6q. Bombée, flus ou moins alongée en pointe alux deux bouts, à bords roulès ca de dans. Ouverture longitudinale, non denrée fur le bord gauche.

Animal. Inoconnu.

9. O v l e o e f, ovale, obtusement prolongée des deux côtés; la lèvre droite renflée, dentée. (ridée).

Ovula oviformis Lamack. Fo "72. O. oram. Bosc. 5. 69. de Roissy. 5 4Ig. 1.

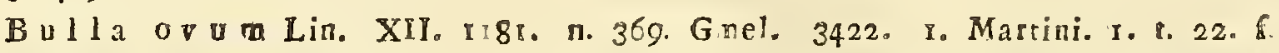
2с5. 2c6. Vulgatrement: oeu de poule, - Se trouve dans la mer des lindes.

* Ovule papyra cée, Ovale, prolongée des deux cotés, la lèvre droite mince, transparente et tranchante.

O vu l a papyracea, testa ovata birostri; labro tenui, acuto.

Cette belle coquille présente la même blancheur que l'ovule oeuf, mais ulle eft plus petite, très mince of transparente. Ia lèvre eft plus ćrartée, et des deux, cotés font plus. alongés que dans $1^{\text {s}}$ ovule oeuf. Sa patrie $\mathrm{m}^{\prime}$ est inconnue. 
3. Ovul e volve, Longuement prolongée des deus cotés; les prolongemens aigus, ftriés.

Ovula volva, Bosc. de Roi sy. ib.

Bulla volva Lin. XII. I131. 11. 3\%O. G nelo ib Mrtini I. to 23 f. $21 \% 218$. Vulgairement: la navette, der ächte Weberftuhl; - Se srouve àx Antilles. Un fuge exe exmplaire de quarante q-atie lignes de lunguur et d'une cono servation parfaise.

4. $O$ v u le bi rostre, Prolongée des deux cotés; les prolongemens lorigs, unis; le bord extérieur plus épais.

Ov bia birostris. Bos: 5 . 70 .

Bulla b. rossris Gmel. Lin -3423. 3. Lister Conch. to I1\% figo 66. Knorr del, 6. 20. 5. - Se trouve dans la ser des Irdes.

3. Ovu le verr q q e use, Ovale tvansversalement anguleuse, arec un tubercule osseux aux deux exirémités.

Ovula ressucosa, Busso 5. 7co de Roisty. 5. 722.

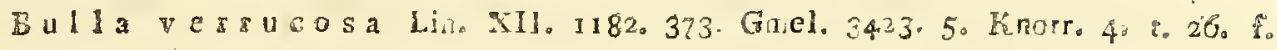

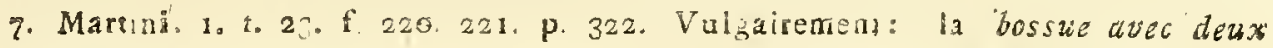
boutons, das Perchen, la citrouille blanchs. - Se trouve dás l'Inde.

6 - 8. Ovu le gibbeuse, Anguleuse avec ur cercle plas élevé; trois individus de differentes grandeurs et coulcurs. Is une eft blevâtre.

Orula gibbosa Bosc. 5. it. de Roissy. ib.

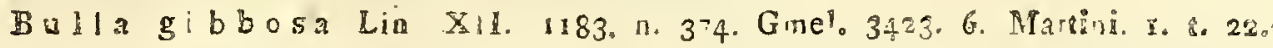

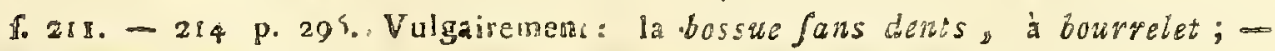
Se sroure au Brisit.

9 - 12 Qudtre autres gibbeuses d'une couleur jaunâtre et d'égale grandeur.

3. 14. * O vule dentée, Ovale; les prolongemens trẻs courts. étant le produit du renflement de la lévre, dont le, bord fit très épais ê finement dentée.

O vula dentat a mihi, testa ovata, margine interius et exterius incrassato, arcte denticulato. Cette ovule eft p'us petite que tcutes les antres, elle n'a que? lignes de longneur sur 4 de largeur. Elle est rrés gib. Beuse, à dos trís éleves, et a des prolóngenens trèz 
courts. qui sont entièrement produits par le renflement de la lèrre, qui a un bord très gros et finement dentelé. Les deux exemplaires que nous possédons sont rouges, couleur de chair, l'une passant au violet; les prolongemens sont intérieurement rouges de cinnabro. - J'ignore so lien nałal.

\section{Tarriére, Terebellum. Lamarce}

\section{Bulla Linnaei.}

Cojuille preaque cylindrique, pointue au sommet; ouverture longitudinale, droite sup ricurement, échancrée à la base; columelle tronquée.

Arimat. Inconiru.

Terebeltuna, testa univalvis convoluta, apice acuta. Apeitura longitudinalis superne argustata, basi emarginata, edentula. Columella truncata. Lam. Anal. du Mus. 1. 389.8.

1. Tarièe $\mathrm{vilb}$ requin, cylindrique, la spire très alongée. Terebellu in subulatum Lamarck. p. 72. de Roissy. 5. p. 423. LVI。 f. 5. Terebellum terebra Bose. 5. 72 .

Bulla re rebellum Lin. XII. I185. n, I78. Gmel. 3428. 22. Martini. \&. p. 190. to 51. f. 568.569 . Vulgairement: Tarrière lisse, Aiguille \& coudre. Se trouve dans la mer des Indes.

2. Tar è re e $\mathrm{n}$ o u blie, Terebellum convolutum, testa tenuis subcylindrica, obtusiuscula; spira nulla; apertura lon* gitudine testae.

Lamarcke Anal. d. Mus. I. 390. I. VI. 226. Pl. 2. fig. 3. Bulla sopita Brand foss. n. 29. t. I. f. 29. Se trouve fossile a Grignon.

3. Tarrièr e fus iforme, Terebellum fusiforme, testa fusiformi cylindracea; spira exquisita obtusiuscula, apertura testae breviore.

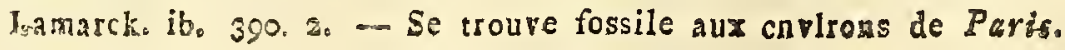




\section{O I i $\sigma$ e. Oliva. L a m a r c k.}

Cozuille subcylindrique, échancrée à sa base. Les tours de la spire séparés par un camado Columelle striée obliquemeut.

Aximal Gasteropode à tête munic de deux tentacules longues, aigacs, les yeux situés vess le milieu des tentacules; un tube au dessus de la tête pour la respiration. Point d'opcreule.

Oliva, testa univalvis, subcylindrica, basi emarginata spirae anfractibus canali separatis. Columella oblique striata.

1. Olive porphyre, Unie; la fpire oblitérée à fa base; la lèvre rétuse dans fon milieu; la columelle ftriée obliquement.

Individu de la plus grande beauté de 4 pouces de longueur. OJira porphyria Lamarck. p. 73. de Roissy. 5 p. 427.

Volut a porphyria Lin. Gmel. 3438. I6. Bosc. 5. 36. Martini. 2. t. 4?. f. 485 486. 498. Vulgairement: Olive de Panama. - Se trouve fur les côtes de l'Amérique méridionale.

2 - 4. Trois exemplaires de la même, presque de la même grandeur; de 3 pouces 7 lignes de longueur.

5 - 9. Six a ut res plus petites.

10. O live porphy re, varieté fingulière avec une côte circulaire élevée au milieu. Isa couleur en est la même comme dans les autres.

11. I2. Olive lettrée, blanche, avec deux bandes et des taches brunes, qui imitent le plus fouvent la lettre $\mathrm{M}$, la fpire distincte et canaliculèe à fa base, la columelle lisse. $\mathrm{G}$ m e lin (dans fon édition du fystema naturae p. 3438. 11. 16. 3.) prend cette olive avec Martini et autres pour une variété de l'Olive porphyre. Mais la forme totale et furtout celle de la fpire qui eft distincte et canaliculée dans la lettrée, et oblitérée dans la porpliy re, nous oblige de la considérer comme espèce distincte La lèvre extérieure en outre eft distarte ou elle a une petite excision près de la base dans la lettrée. Elle eft au contraire attachée dans la porphyre. 
13-16. Quatre Olives Iettré es à taches plus raprochées et à bandes moins distantes.

8. 5. De ux olives lettrées à lève très renflée et plus distante que dans les autres.

Les figures appartenantes à la lettrée font:

Martini. 2. t. 45 . f. $4 \% 6$. $47 \%$.

Knor Delices t. 3. tb. 2. f. 3 .

19. 20. L’olive nègre, unie; la base de la fpire recourbée. la columelle obliquement ftriée.

OIiva fusca m.

Voluta oliva Lin. Gm. 2499 ' \%. Borc. 5. 3\%. Martinio 2. to 45 . E. 472. At33. Knorr. 5. to 28, fo 6. - - fe troure dins la mer des Indes:

On considère comme variétés de l'Olive riègre:

21. B. l'Oli ve à robe brune plus claire avec des raies transwer. sales plus foncées.

22 - $25 \cdot \gamma \cdot$ IPO Live à robe brun-clair passant au rouge ou au jaune.

26. S. lo live à robe brune avec une bande ou zone, au milieng, tachetée de noir.

27. 28. $\varepsilon$. I'O I ive brene à ftries longitudinales plus foncées:

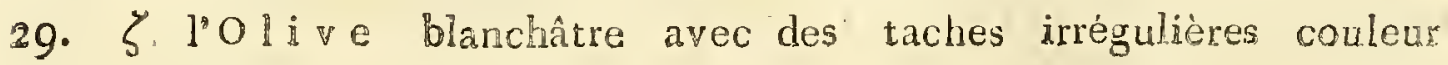
d'olive.

30. 9. l'Olive verdâtre avec des desseins en zigzag.

31. - 42. Douze olives qui appartiennent à la méme ra. rété, et qui présentent plusieurs nuances dans le dessein.

43. Ol i ve bianchâtre avec des ftries brunes très rapprochées en zigzag.

44. 45. O I i ves blancheseverdâtres avec deux zones foncées.

46. - 47 Olives blanches avec des desseins violets en zigrag. Deux Individus differens. 
53 - 59. 3 uite d'Olives dont les desseins en zigrag forment des zones réticulées; 15 Individus.

83 - 89. Olives tout à fait blanches; fept individus de différente grandeur. Celles-ci paroiffent forme: l:espece de Gmelin et de Bosc qu'ils appellent coluta wiva. Io crois que toutes les olives qui présentent des diffirences essentientielles dans la formation de la fpire ou de la columelle doivent être envisagées comme espéces distinctes Il fandra chone rañger plasieurs parmi celles que $G m e l$ i n allègue comme variétés de Voluta olua. Je considère comme espece diftinote ê nonvelle.

90. L'olive bossue, Ovale unie, le fecond tou" he la fpire enfoncé, trois plis distincts de la columelle, dunt le premier très élevé.

Oliva plic at a mihi. Je n'en connois pas de figure.

Elle eft ovale, blanche ou verdàtro, ponctuée de brun de différente manière. La lèvre eft épaisse, distante dars toute fa longuen:

Dle eft de la grandeur de l'olive nègre.

91. V a riété de la même, jaune, ponctnée de brun.

92. Variété de la même, verdâtre, poractuée de brun.

La patrie en efl inconnue.

93. Olive utricule, Alongée, unie, la fpire faillante.

Oliva utriculas; mihi.

Voluta utriculus $L$ a Gael. 344r. ก. 19. B)sc. 5. 37. Martiai, 2. L. 49. 50. f. $539 .-548$. - S: trouve dars la mer cles Indes.

94. 35. La mề me d'un plus petit volume.

c6. Variété dans la couleur, blanche avec deur bandes jaunes.

97. 98 . Deux antres irdividus gui présentent une variété de la coulevr; i's font ftriés longitudinalement en rouge.

99. Olive hiatule, Mince? le dos cendré, maculé, le dessus inégal; l'ouverture ample, la columelle dentée à fa base. Oliva hiatula.

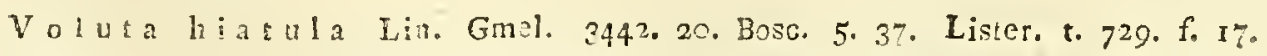
Martini. 2. t. 50. f. 555 - Se trowe fur les côtes d'Espagne. 
I00-II Douze Individus qui paroissent appartenir à la même olive. Ils diffèrent dans le coloris.

I I 2. - I r 4. O l i ves - h i a tu l e, Trois individus, les feules dont la lèvre foit très mince; à ceux-là fe rapporte furtout la figure de $L$ is $t$ e $r$.

115. Olive jaspée, Blanche ponctuée de brun, de violet et de vert; les tours de fpire avec une fascie maculée $\dot{a}$ leur base.

Olive jaspidea.

Voluta jaspide a Gmel. 3442. n. 2I. Bosc. 5. 38. Lifter 726. I3. Martini. 2. t. 50. f. 556. - Se trouve fur les cotes d'Espagne

I I6. - I 30. Q a inze individus qui paroissent appartenir à la même espèce, ne présentant que des différences de dessein et de conleur.

I3[. I32. Olive c a r n é ole, Orangée, avec des fascies bleues; la fpire applatie? et l'onverture blanche.

Oliva carneola.

Voluta carneola Gmel. 3443. n. 24. Bosc. 5. 38. Marsini. 2. 1. 46. f. 495 . On ignore fon pays natal.

133. Olive Girol, Alongée, lisse, la fpire courte et lisse, In bouche très onverte et violette.

O Iiva guttat a mihi. Elle a été figurée par Lister 72 . 6. Martini 2. p. 161. t 46. 493. 494. le Girol d’Adanson p. 6[. Pl. 4. f. 6. - La partie en elt inconue.

I34. I35. Olive is pud ule, unie; la fpire proéminente; la lèvre aves un feul cordon; la columelle obliquement ftriée. Oliva is pidula.

Voluta ispidala Gmel. 3442. n. 23. Marrini 2. t. 49. f. $522 . \quad-530$. Busc. 5. 38. Deux petits individus qui parnissent être des variétér.

136. O live a longée, Alongée, à fpire très longue à lévre distante.

O i va elongata. 
137-143. Sept individus différens en couleurs qui appartiennent à la mème espèce.

G meli n a considéré certe olive comme une variété de l'Utricule, (Mara tini 2. 50. f. $549-554$.) ma's à ce qu'il me paroit, elle dost furmer une espéce distincte, vu fa proportion alongée et cgliudrique; l'autre étant veutue。

\section{Ancille. Ancilla Lamarck.}

\section{Anaulax de Roissy (*). Voluta Lin naei.}

Coquille oflongue, à fpire courte, non canaliculée. Base de l'ouverture à peine échranctée versante. Un renftement ou ua bourselet oblique et calleux au bas de l\&

Animal inconnu. columelle.

Ancible Testa univalvis, oblonga, fubcylindrica; fpirâ breyì, non canaliculata. Aperos tura basi vix crnarginata, effusa; Varix obliquâ, callosa ad basin columellae. Lamarck. An. d. m. I. 474. 10.

I. Ancille cín n momée, alongée, cylindracée, mucronée. très mince, et lisse; le bourrelet, qui commence très haut. lisse.

A ncilla cina mom e a Lamarck. p. 73. Voluta Martini 2. p. 362 . t. 65 . fig. 731.732 .

Ia rôtre elt violette avec trois zones blanches, aussi le dedans eft violette; mais en la tenant contre le jour elle paroit être brune, brun de canelle. $C^{\prime} \in$ ft peut être la rause du nom fpécifique de M. Lamarck. - Se trouve á Coy rine.

2. Ancille cochenille, ovale, fpire obtuse, firiée longitudinalement, le bourrelet plissé, terminant la columelle. A ncilla coccinea, mibi; - des mers ocridentales. I_'espèce que $j^{\prime}$ ai appellée d’après la couleur quniqu'elle foit peu constante dans ce genre fe caractérise rependant par fa forme comme espèce distincte. Elle a une fpire très

(*) Mr. de Roissy a cru devoir charger le nom du genre Ancille de Lamarck parceque Geoffr o y aroit employé le nom d'Ancille pour dos guacr les pateiles tluviatules. Anaulace veut dise fans canul.

$$
2^{*}
$$


obtuse, la lèvre extérieure irrégulièrement ftriée, peu mince et le bourrelet qui fe trouve presque en bas de la colur melle eft legerement fticée ou pliée. Ille a I pouce 7 lig. nes de longueur. Il paroit que la figure de $\mathrm{Ch}$ em $\mathrm{n}$ t 10. f. I38I. appartienne à la même espéce quoique la fpire eft moins prononcée dans la nôtre.

3. - E. Ancille lisse, alongée, cylindracée mucronée, le b-urrelé élevé et distinct, laisøant la base de la columelle, qui eft lise, libre.

Ancilla laevigata, mili. Elle eft verdâtre, vert dolive, ou brunâtre à zones très larges plus claires. Quatre individus qui présentent des différences de couleurs. No. 5. et 6 a beaucoup de ressemblance avec les figures 728 et 729 de Martini. t. 2. to 65. qu il a décrit, fous le nom de voluti.

7. Ancille bullée, ovale, très ventrue, fpire applatie, lèvre très baillante, bourrelet large mais peu élevé, faisant la base de la columelle, bordée par un petit renflement en lizière.

A n cill a bu 11 at a, mili. Conus bullatus Linnaei; Martini.

2. t. 65.726 .727 .

8. Ia même bullé e depouillée présentant on outre une différence de la fpire qui eft élevée.

Espèces fossiles.

9. Ancille à gouttì̀re, Ancilla canalifera, testa cylindracea, mucronata: labro antiquo canalifero; callo columellae fubplicato.

I a ma rck, Anmal. d. Miss. I. 475. 4. Elle fe trouve fossile à Grignoaz en France.

Volute. Voluta. Iin. La m.

Coquille cvale, plus ou moins ventrue, à fommet obtus ou en mamelon, a base eschancrés et fans caral. Columelle chargéc de plis, dont les inférieuts font le plus gros ou le plus longs. 
Animat, Gastéropode à tête munie de deux tenacules pointues: les yeux à leur base extéricure. Bouche en trompe alongée, cylndrique et retractile, garnie de petites dents crochues. Ua tube pour la répiration, faillant obliquement derrière la tête. Pied fort ample. Puins d'Opercule.

Folzsta, univalvis, ovata, fubventricosa; apice papillari; basi emarginata. Columella plicata: plicis inferiorbus majoribus vel longioribus. Lam. Anal. du Mus. I. 475. II.

I. Volute musique, emarginée; les tours de fpire avec des épines obtuses; la lèvre unie très epaise.

Voluta musica. Lin. Gme1. 3460. g6, Martiaj. 3. t. g6. f. 925. Lamarck. p. 74. Bose. 5. 8. de Roissy. 5. p. 436. t. 56. f. 8. - Se trouve fir les cóes d'Amérique.

2. Volute musique, exemplaire de la même beauté, mais qui, quoique plus large, eft cepersdant plus court.

3. Volute musique femblable dans les dessein mais a couleurs plus foncées.

4. - 8. Volute musique, cinq individus à points intermédiaires plus petits.

9. Volute masique, variété à épines très élevées et très pointues.

10. Volute musique, variété à épines très obtuses et très bases et à lignes transversales presque effacées.

I I - 13. Volute mus ique, 3 variétés rougeâtres à points intermédiaires très petifs.

If. Volute musique, varièté très jolie à, trois zones de lignes transwersales, avec des points intermédiaires très regulièrement placés.

15. Volute musique, variété fans lipnes transiversales avec des points à queue comme les notes de musique; les épines de la fpire font courtes mais très pointues.

16. Volute musique alorgée, variété très intéressante, qui me paroit faire une espèce distincte, et par fa forme alongée et par les bourrelets de fa columelle qui font très 
élevés et au nombre de cinq. Il y en a toujours d'avantage et moins élıvés dans la vraie musique.

Knorr Del. 3. 12. 1. et Chemnitz 10. p. 149. to 149. f. 1402 1402 l'ont représcntée comme une variété de la volute musique.

17. Volute hebrai u e, Emarginée; les tours de fpire avec des épines emoussées; la columelle avec cinq gros plis et trois petits.

Voluta hebea ica Lin. Gm. 366r. n. 93. Bosc. 5. 60. Natre exemplaire eft plus petit et présente aussi quelque différence dans le dessein decelles dout les auteurs citent les figures pour cette espèce. Elle le touve dans la mor des Indes et aux Antilles.

18. Volute chauve-souris, Emarginée; les tours de fpire aigus; la columelle à quatre plis; la lèvre unie.

Vol uza ves pertilio Lin. Guel. 346I. n. 97. Bosc. 5. 60. Chemaiz. XI. f. 1699 .

19. - zI. Vol. chauve-souris, trois variétés á desseins en zigzag dont la couleur eft différente.

* Volutes ventrues.

22. 23. Vol u te $\mathrm{nacelle}$, emarginée; les tours de fpire canaliculés en leurs bords; la columelle à quatre plis.

Vol us a c y mb i um. Lin. Gmel. 3466. n. II 4. Volute yet? Bosc. 5. p. 65. Martinio 3. ti.70. f. 764. - Elle elt mangée par les negres des côres africaines et américaines.

94. Volute nacelle, variété à spire mamelonée et à lèvre plus approchée.

25. Volute in a c $11 \mathrm{e}$, variété d'un coloris brun, à lèvre plus écartée.

26. 'Volute éthiopique, Emarginée; la spire couronrée d'épines en voûte; la columelle à quatre plis.

Vollota a ebhiopiea. Lir. Gm. 3455. n. Ir3. Bosc. 5. p. 64. Martini. 3. 8. 73. fo 77\%. Vulgairemont: Couronnes d'Ethiopie: - Se sroure sur la côte orientale de l'Afrique.

2\%: Volute citrinée, Emarginée, la spire unie, la columelle à quatre plis. 
Volut a citrina mihi. Cette belle coquille jaune à taches brunes qui forment deux zones parallèles et doit Martini paroit avoir figuré un petit exemplaire vol. 3. t. 72. f. 772. forme une espèce bien distincte parmi les volutes ventrues en ce que la spire lisse et très base mais cependant composée de trois tours eft cachée d'un coté par la lèvre droite et en ce que la columelle prćsente quatre plis difincts. Elte se trouve dans les mers des Indes. Nitre exemplaire a quatre pouces neuf lignes de longueur sur trois poures de largeur.

28. Volute philippine, Emarginée; la spire unie; la co. lumelle à trois plis.

Voluta olla Lin. Mus. Lud. Ulr. p. 599. n. 243. Gmel, 3466. n. I I5. Volute philin Bosc. 5. p. 65. Martini. 3. t. 7. f. 766. - Se trouve dans l'océan Africain, Anéricain et 1udien. Un exemplaire de la première grandeur, de 3 pouces 6 lignes de longueur.

99-3I. Volute philippine, trois individus plus petits qui présentent une legère différence dans le contour de la lèvre droite.

Mitre. Mitra Lamarck.

$$
V \circ l u t a \text { L in } n \text { a e i. }
$$

Coquille turricule ou fubfusifurme, à spire pointue au fommet, à base échancrée et sans canal. Columelle chatgée de plis donr les inférieurs sont les plus petirs.

Animal inconnu.

Mitra, Testa univaluis, subfusiformis, apice acuta, basi emarginata; canali nullo; columella plicara; plicis inferioribus minaribus Lam. An. du Mus. 2. 57. I2.

1. Mitre épiscopale, Emarginée, unie; les tours de spire non dentés en leurs bords; la lêvre denticulée.

Mitra episcopalis Lalarck. p. 74. de Roissy. 5. p. 44t.

Voluta e piscopalis Lin. Gm. 3459. n. 94. Martini. 4. t. I4t. f. 1360.

- des Indes. 
2. Mitre épiscopale, exemplaire plus petit mais de couleurs bièn vives.

3. Mitre.papale, Emarginée, ftriée transversalement; le bord des tours de fpire et la lère denticulés; la columelle à quatre plis.

Mitra papalis; exemplaire de grandeur extraordinare, de presque cinq pouces de longuen.

Voluta papalis Lin. Gm. 3459, n. 95. Bosc. 5. p. 59. Martini. 4. t. 14.7. 1. 1353. 1354. - Se troure clans la mer des Indes.

4. Mitre papale petite variété garnie de zones, ponctuées trés profondement.

Chaque fpire présonte trois zones de points profonds, la lèvre n'ef pas dentée, les plis de la columelle font distiacts ar nombre de trois, le quarième n'eft qu'apparente. lans l'autre n. 3. qui eft lise qui ne fait vois aucun point cufouce, il y a a plis distincts à la columelle et le cincuieme els apparent. Ces différences ne font ellos que cause de l'age?

5. Mitre cardinale, Emarginée, ftriée transversalement; blanche, avec des taches, conleur de paille, dont plusieurs rangées en échiquier; la columelle à cinq plis. Mitra cardinalis; de deus ponces de longueur. Voluta cardinalis Lin. Gmel. p. 345s. 2. 3. Boss. 5. p. 58. Marini. 4. 1. 147. f. 1358. 1359. - Se tronve dans ia mor des Frales.

6. Mitre cardina le d'une belle grandeur, de deux pouces neuf lignes de longreur, et gni cependant ne présente que anare plis à fa columelle.

7. Mitre percée, Frraginé, frićo, percée de trous; la leve denticulée, Ja columelle à cinq plis.

Mitia peltusa. Cxemplare de trois pouces denx lig. nes de Jongueur.

Voluta pextusa, Lin. Gne1. 3458. n. 92. Bose. 5. p. 58. Martini. 4 t. 547. f. I36r. - Sa liouve dans la mer des Indes.

8. - IO. Mitre percée, trois exemplaires plus petits qui. présentent quelgue différénce dans la couleur et dans l'arrangement dés côtes formées par des points enfoncés. 
11. Mitre fendue, emarginée, avec des côtes longítudinales, furmées par des plis; des stries transverses fines, et la co. lamelle à trois plis.

Mitra subdivis a.

Voluta subdivis a Lin. Gmel. 3453. n. 133. Bosc. 5. p. 48. Chemnitz. 10. $t, 15$ r. fo 1424 . se trouve das la mer des Indes.

12 - I5. Mitre fendue, quatre variétés différentes en desseins et en conleure.

16. Mitre caffre, emargiaée, cylindrique, unie; les tours de spire plissés et striés; la columelle presque à quatre plis. Mitra caffia.

Woluta caffra Lilio GmeJ.345I. n. 51. Boce. 5. p. 46. Martini 4. f. I48. f. 1369 . 1370 . - Se erouve dans la mer des Indes.

I. Mitre caffre, exemplaire à peau naturelle et par consequent plus pâle.

58. Mitre plic aîre, emargirée, anguleuse; les angles antérieurs presque épineux; la columelle à quatre? plis.

Mitra plicaria.

Volutá plicari?. Lin. Fmel. 3452. n 55. Brsc. 5. p. 47. Martini. 4. \& 148. f. 1362. 1365. - Se trouve dans la mer des Indes. Norre exempiaire ne piésente que trois plis diftincts de la Culumelle.

19. Mitre hérisson, emarginée, furiée, transversalement rugueuse, la columelle perforée, à quatre plis; la lèvre crénlelée.

Mitra scabriuscula.

Voluta seabriuscula Lin. Gmel. 3450. 48. Bose. 5. f 45. Martini 4. t. 149. f. I388. 1389. a391. Se trouve dans la mer des Indes.

8. Mitre nu bile, presque entière, mie, ftriće transversalement de rouge, sale; la lévre crenelée; la columelle à quatre plis.

Mitra nubila.

Vol a ta n ubila Lin. Gmel. 3450. n 143. Bose. s. p. 4h. Martyn. Univers, Cench. 1. to 23. - Se truuve dans la mex du Such. 
2I. 22. Mitre g e not, emarginée, sillonnée longitudinalement, striée transversalement; la columelle à quatre plis; la lèvre unie.

Mitra sanguisuga.

Voluta sanguisuga Lin. Gm. 3450. n. 50. Buse. 5. p. 45. Martini. A. ז. 148. f. 1367 . 1368. 1373. 1374. - Le jies natal en cft inconon. L'un des deux exemplaires ne prèsente d'aurres différences que des coulcurs plus foncées.

23. Mitre more, un per émarginée, cylindrique, unie; la columelle à trois plis.

Mitra morio.

Voluta morio. Lin. Gmel. 345 I. n. 52. Boss. 5. p. 46. On ignore le lieu où elle se trouve.

24. Mitre noire, emarginée, noiratre; les tours de fpire applatis; la columelle à quatre plis.

Mitra nigra.

Vo, lu a n igra Lin. Gm. 3452.a. 132, Bosc. 5. p. 48.

Chemnitz 10. t. I5I. f. I430. I43I. Notre exemplaire eft plus petit et plus alongé que cette espèce y eft repsésentée. Elle fe trouve daus la?mer de l'Inde et de l'Afrique.

25. Mitre renardine, emarginée, presque anguleuse, fans épines, ftriée transversalement; la columelle à quatre plis; la gorge ftriée.

Mitra vulpecula.

Voluta wulpecula. Lin. Gm. 345T. n. 54. Bosc 50 47.

Marsini. 4. 4. I48, f. I35. - Se trouve dans la mer des Indes.

36. Mitre ensanglantée, emarginée, fasciée, transversalement ftriée, avec des côtes longitudinales noueuses ma: culées de rouge; la columelle à trois plis.

Misra c suentata; - Voluta cruensata Lin. Gn. Les poinis rou ges font aussi regGlièrement placécs que dans l'aiguills. Martini. 4. to $15 \%$

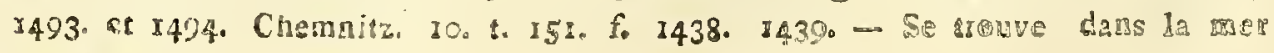
des Iodes. 
27. Mitre raboteuse, emarginée, granuleuse, ftriée en fautoir, avec des rôtes longitudinales, fasciées de brun, la columelle à.cinq plis.

Mitra exasperata. Lin. Gm. 3453. 135. Bosc. 5. 49. Chemnitz yo, t. Igr. f. 1440. 244. - Se trouve dans la mer des Indes.

28. Mitre turiculée, emarginée, fillonnée lóngitudinalement, à fillons écartés, ftriés transversalement, la columelle a trois plis.

Miera er r riculata, testa turrita emarginata, longitudinaliter fulcata, coftis diftinibis, tancyersim Ariata, columella triplicata.

Une tiès helle mit e jaune a tours de fpire bodés de trun foible. Les côte: qui garussent toute la coquille, funt larges et tzès écartées, un peu courbée au milicu.

Es pèces fossiles.

29. Mitre marginée, Mitra mrginata, ovata, laevius. cula; anfractibus marigine variculoso crenulatoque fubduplicatis.

Lamarck. An. du Muséua. 2. p. 53. 12. 3.6. t. 44. f. 7. - Se trouve fossile aे Grignon.

30. Mitre plicatelle, Mitra plicatella, fusiformis, laevigata, anfractibus margine fubplicatis, columella 4 plicata. Lambrik. 2. 58. 4. tom. 6. tab. 44. F. 8.

\section{Marginelle. Marginella I.amazck.}

Coquallte ovale - oblongue, lisse, à spire courte et à bord droit rebordé en dehors. Base de l'ouverture plus oll moins échancrée. Iles plis à la solumelle.

Anifral. Gaftèropode à deux tentacules pointues, portant les yeux près de leur base cxtérieure. Bou he en trompe retactile. Un tube se prolongeant au dessus de la têce four ia resfiration. Le disque ventral dépassant postérieurement la coquilie. Point d'opercule.

Marginella. Tecta unira'vis, orato-oblonga, laevis, spirå brevi. Labrum exius calloso- mnrginatum. Apertura basi subemarginata. Columella plicara. 
- 3. Marginelle porcela ine, très entière, unie; la spire unie; la columelle à quatre plis également diftans; la lèvre bossue, bourrelée, dentée.

Marg inella glabella, Lamasck p. 75. Voiuta glabella. Gmel. Lin. 3445. n. 32. Bosc. 5. 40.' Martinio 2. to 42. fo 429. - Se trouve dans la mer des Indes.

A-6. Margine $1 \mathrm{le}$ b l e ue, bleuâtre, unie, la spire plus ou moins pointue, la columelle à quatre plis, dont les deux supérieurs sont plus rapprochés; la lèvre bourrelée, lisse.

In áginella gla uc a mihi. Das hangefreifre zelg, dic Eyerschnecke. Knorr. IV. t. 23. et 27. fo I. Martini. It. p. ro6. T. 42. f. 424. 425 . Martini indique des lèvres oranges, elles sont blanghes dans nos trois exemplaires. - De la mer des Incles.

7. 8. Marginel le bossue", bleuâtre, ventrue, la spire très courte, la columelle à cinq plis, la lève fortement bourtelée, lisse.

Marginella ventricosa. m. Ia patrie en est inconnue.

9. 10. Marginelle bobi, la spire émoussée et ombiliquée; la columelle avec sept plis, la lèvre crenelée.

Marginella persicula. m. Voluta persicula, Lin. Bosc. 50 40. Mar\&ini. 2. to 42. f. $419-422$. Elle varie beaucoup. Celles-ci sont rayécs de rouge.

II - 12. L a $\mathrm{m}$ ê $\mathrm{m}$ e ponctuée de rouge.

13. 14. Le Concombre à points bruns, (Martini, 2. t. 42. f. 432. 433.) est considéré comme variété de la porcelaine.

15-16. Variét é de la porcelaine à spire oblitérée et à lèvres à taches oranges.

İ - I8. V ariété de la même à ftries transversales violettes.

19. Semence de la même dans une petite boite.

Espèces fossiles.

20. Marginelle en ov ul e, M. ovulata, Lamarck Anel. d. m. I. 2. 61. 1. VI. 226. t. 44. f. IO, 
Cancellaire. Cancellaria Iam.

cogarille orale ou fubtarriculée à bord droit filloné intéricurcment. Base de l'ouverture presqu' entière et un peu en caial。 Quelques plis compsimés ou tranchas fur la colume.le.

Antmal inconnu.

Canceliaria, Testa univalvis, ovata vel fubturnta; labro intus fulcato. Apezturac basis integriuscula fubeanaliculata. Plicae compresso-aoutâe ad coilumellam.

I. Cancel la ir e réticulée, un peu fillonnée en fautorir la lèvre intérieurement ftriée; la columello presque perforée. Cancellaria reticulata, Lamarck p. 76. Voluta cancellata Lin. Mâninio 3. I2 T. f. Iro7. Born. t. 9. f. 7. 8.

2- 6. Variétés de la même, foit par la difference de la cou leur, foit par l'arrangement de fes côtes.

7. 3. V V a r i ét és à plis oblitérés fur la columelle.

9. - Ir. * Cancellaire mitroide, fillonée, les fillons larges et lisses, les plis de la Columelle très courts.

Cancellaria mitroides mihi.

Espèces fossiles.

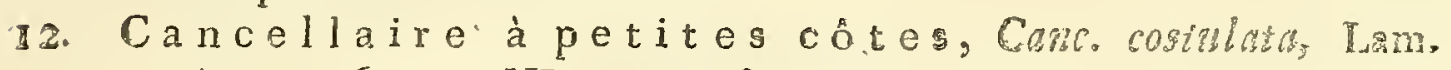
A.s. 2. 63, 1. VI. t. 44. F. IE.

\section{N a s s e. Nassa L a rito}

\section{Buccinum Lin:}

Cogatile avale. Ouverture fe terminant inférieuremcnt par une échancrure oblique qui remonte postérieurement. Eord gauche calleux, formant furla columelle qualil re couvre, une base ou un pli transverse dans fa partie fupérieurc, et ayant fa base obliquement tronquée.

Nossier. Gastéropode à disque rentral tiargi et tronque antérieurement, et fe prolor: geant au delà de la tếte. Deux tentacules pointues portant les yeux daus leur partie moyeane, Ua tube au dessus de la têre, formé pas le manteau. Listo 8. 975. f. 30 , 
1. Nase casquillon, ftries transverses, et plis obliques terminćs près la future par de gros tubercules: cốtes longitudinales lisses.

Nas a a r ularia, Lamarck p. 76. Buccinum arcularia Lia. Rosc. Mage tini. 2. t. 4I. f. $4090-4$ I2. Bose. 4. pl. 36. f. 2. 3.

2. Le casquillon sans le pli transverse.

3. * $\mathbf{N}$ asse gla nule us e, Pli oblique lisse, cêtes longitudinales grainues.

Nassa granulata mihi.

4. Nasse Iisse, pli oblique lisse, courrant à peine la columelle, côtes élevées seulement aux demiers tours de la spire.

Nassa laevis $\mathrm{m}$.

5. La même sans le pli oblique.

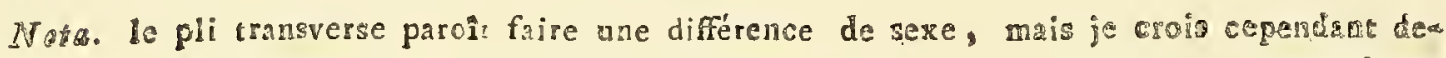
voir conserver le genre de Mr. Lamarck, ru que la columelle a la mêtme furme que dans les nasies à plo, forme qui eit propre aux Nasses de tamarkik ef qui he se trouve pout dans les Buccins proprement dits.

\section{I i c o r n e. Acantbina.}

Coguille ovale. Onverture se terminant inférieuręment en un canal tress court, obligue, pea échancrè à l'extrémite. Bord gaushe calleux, formant un bourreles difinat de 12 Coltuelle. Ièrre droite épaisse, munie près de la base d'une épine tiss longue.

Licorazier inconnu; - mais cette longue épine faik soupçonner une ftructure propre a cetze anima!, et bien différenæe soit des pourpres de Lamarck soir des Buccins.

Les Liscormes fo distiaguent des pourpres patr le bourrelet de lacolumelle et parcegue da lórre droite uta pas d'ezcision ou de canal à fa base.

1. 2. I a li c o I n e tuilée, brune, les côtes inégales, garnies d’écailles tuilées; la lèvre droire crenelée.

Aca othin a iubricata m. Buccinum monodon, Pallas Spicil. yo. p. 33. t. 3. f. 3. 4. - Dez bonnes figures. Voy. Koorr. IV. to 30. f. x. Ficgeu\&us T. 
2. ib. \%. f. 2. Martyn's Uniy. Con hol. T. I. f. 1e. T. 2. f. 50 . Bucc. Calcap; Chesnitz. 10. t. r54. f. $14 \%$. 470 . dis parages magellaniques. - Bose dans fon ouvrage d'ailleuas très eftimé fur doıble adhibition de la licorne tui. lée. Une fois clle représ fite Buccinum monoceros Voy. T. 4. p. 268; une autre fois Purpura monodon, Ton. 5. p. 2\%.

3. La 1 i corne t u ilée fossile. Elle ne fe distingue que par la lèvre droite plus mince, rayée en dedans de violet, et par fon aspect fossil.

4. La li corne à côtes, jaunâtre, les côtes inégales, les plus élevées tranchantes, la lèrre droite plissée en dedans. Acanthia a cosiasta milhi. Buccinum narbal Eose. 4. 268.

5. 6. Variété de la même plus peitre et noirâtre; deux individus de la même grandeur mais dont l'un a l'épine beau. coup plus longue que l'autre.

\% La licorne lisse, lisse et ̊̀ côtes oblitéréon, la lère droite lisse, l'épine très longue et visible le loug de la lèvre droite.

Acanthina laevigata mihio

8. La $\mathrm{m}$ ê $\mathrm{m}$ e depouillée.

Pourpre. Pupura Bruguiere.

Buccinum Lin.

Coquille ovale, le plus fouvent tuberculeuse ou épineuse. Onverture fe terminant inférieurement en un canal très court, oblique, échancré à l'extrératé. Colusielle wue, appiatie fur tout inférieurement et finissant en pointe à la base.

Pourprier. Gastéropode à disque ventral siliptique, plus court que la coquille. Deux tentacules pointses, portant les yeur dans leuz parke moyenne extéricure. Manteau formant pour la respiration un tube qui passe obliquement au dessus de la tête. Un cpercule cartilagineux ef femi-lunaire. Aclars. Sencg. t. \%. f. I.

1. 2. 3. Pourpre persique, ftriée, tuberculeuse, la lèvre crénelée; la columelle appiatie.

Purpura persica. Biguguiere, Lamarck, Bosc. - Euccinum persicum Lin. Martini 3. t. 69. f. 760 . - Se trouve dans la mer des Indes. 
4. 5. La m é mo depouillée et polie.

6. \%. Pourpre pakel, épineuse; la lèvre crénelée extérleu: rement, fa columelle en faucille.

Pu в pura patula Besc. 5. 25. - Buccinum patulam, Lin. Martiaio 3. to 69. f. $75 \cdot 759 \cdot$ - fur les cô:es de l'Afrique et de I'Amériquze.

8. Variété de la même à.épines plus courtes et à côtes intermédiaires plus élevées.

D. Porpre fakem, ovale, a tubercules obtus; I'ouverture fans dentelures, la columelle frriée transversalement, et la lèvre droite profondement fillonnée.

Puspura mancinella, Bosc. 5. p.28. p. 37. f. r. 2. Murex mancinelia Lin. - Martini 3. to 100. f. 963. \&. 10r. f. 966. 968. Adanson. 7. Io Se trouve fur les côtes d' Afrique et dạs la mer des Indes.

Notre exemplaire est de la premiere grandeur, de 2 pouces et demi de longuerr:

10. Pourpre Iaborin, ovale, friée, avec quatre rangs de tubercules presque épineux; l'ouverture ftrice transversalement.

Purpura hippocastanea Bosc. 5. 28. Murer hippocastanum Linn. Adanson pl. 7. F. 2. Martini 3. 1. 99. אิ 945. 946. - Se trouve dans la mes des Indes ef fur les cô:es de l'Afrique.

Nota. Il ne faut pas confondre avec les espèces décrites, par fuite de la fimilitude des noms, celles appellées pourgres par Dargeuville, Eavanne et autres Conchyliogistes frarsois, lesq elles entrent dans le gcare, rocher",

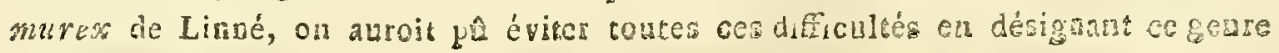
par un autre nomo

\section{Buccin. Bucsintem $\mathrm{I}$ a mark.}

Coqzille ovale ou alongé. Ouversure oblongue, échnorke inférieurenent et fans canal. Echancrure découverte ansérjeugmens. Colemelle pleines fans applakissement a $f_{a}$ base.

Fascinier: Gasteropode à pied elliptique, pirs coure que la coqualle. Derx tentaculcs coniques, portant les yeux à leur base extérieure, Marteal formant pour la 
réspiration, un tube qui passe par l'échancrure de la base de la coguille er fe prolonge au dessus de la tête de l'animal. Un epercule cartilagineux.

I. Buc c in r a yé, lisse, blanc, marqué de lignes branes trans. verses; le bas de la lèvre droite gani de dents épineuses.

Bu ccinum glans Lin. Bosc. - Martini 4. t. 125. I196-1198. - Se trouve dans la mer des Indes.

2. Buccin ponctuée, qui paroit une varióté de la précédente.

3. B a c c in lisse, oblongue, à tours peu distincts, blanchâtre le sommet ceinturé de violet.

Buccinum la evi gatum Mrartigi. 4. to $12 \%$. f. 1216. $x$. c'est fans doute la même coquille que cet auteur a aussi figurée fous le nom de $N$ assa labvis ib. t. I25. f. I193.

4. 5. * Buccin porcelaine, oblongue, très épaisse, blan. che, à stries circulaires brunes et très étroitement placées, la bouche très blanche.

Buccinum agat hinum mihie - Der braungestreifte Bauernjunge Mĩartini. 3. \&. 120 . f. x104. 1105 . Vulgairement la bouche de lait. Deux individus, dont l'un depeuillé, des Indes orientales.

6. * Buccin r ubanné, oblongue presque fusiforme, brun, à fascies transversales plus foncées, dont deux regulièrement tachetées de blane.

Buc c i n in fasciatum $m$ ihi. Les taches blanches triangulaires et en forme de fleche, qui imire un peu la barbe d'une plume a fans doute produit le nom de Hahnenfeder, Buccinum pennatum de Martini. 4. 5. 12\%. f. $1218-20$. Se trouve fur les côtes de Famaiqules et de l"Ascension.

7. Bucein sillonné, fusiforme, angulé, blanc-jaunâtre, ḋ stries transverses élevées. brunes.

Buc cin um Vadacum Lin. Nassa vndosa Martini 4. t. 122. f. 1126. II27. des Indes.

8. Buccin t uberculeux, roussatre, couverts de plusieurs rangs de terbercules arrondis.

B u c inum papillos u in Lin. Bosc. Martini. 4. t. 125. f. 12c4. 1205. Se trouve dans la mer des Irodes. 


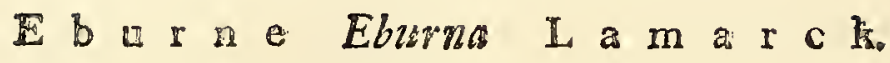

Buccinsm. Iinné. Bosc.

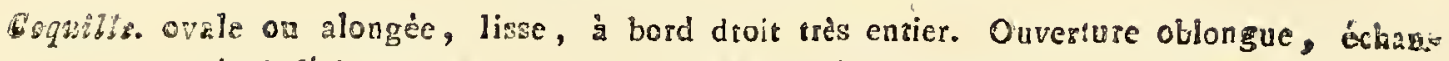
crée inférieurement. Columelle ombiliquée fubcanaliculée à $f_{a}$ base.

Animal inconn.

I. 2. E b u rn@ ja ư ât re, très lisse, ombiliqué, jaunâtre sans sutures distinctes: (tour inférieur de la spire marqué extérieurement $d^{2}$ un sillon).

Eburna flavida Lamarck. p. 78. Buceinum glabratum Lin。 Bosc, Marta 4. to I22. fo III7. Se trouve aux Indes.

3. Eburne c an a li cul é e, ombliquée, lisse, tachetée de jaune; le bord fupérieur des tours de la fpire creusé en canal de vive arête.

Eburna fpirata mihi. Buccinum fpiratum Lin. Boøc. Marto 4. t. r22. fo III8. - dans la mer des Indes.

G. E b u n e de Chemnitz, ombiliquée, lisse, blanche, tachetée de brun ou de pourpre, les tours de fpire distincts mais arrondis.

Eburna Chemnitziana mihi. Linné regardoit cetre belle coquille comme une vaziété de la précedente. Mais elle en différe totalement par les caractères Enoneds dans la description et furrelut par l'omblique non denté. Chemnitz l'a

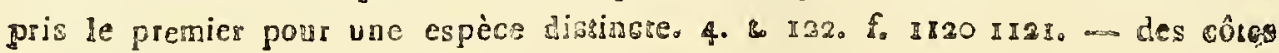
de la chine ef des Istes roisines.

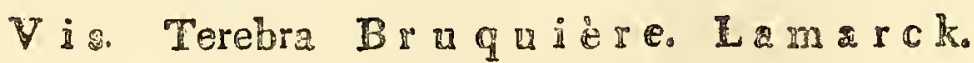

\section{Buccinum Einn.}

6oq. qurriculẻe. Ouversare echanesée inférieurement, et au moins deus fois glus course que ta coquille. Base de la columelle torse ou oblique.

Arziral. Gasteropode rampant fur un disque ventral beaucoup plus cours guve la coquille. Deux tentacules pointues, portant les yeux à leur base exterieure. Manteav formans un tube qui fort par l'échancrure de la coquille et fe dirige oblique

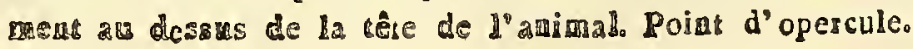


I - Vis, ma culée, les tours de fpire unis, fans fillons intermédiaires, fans dentelures, fasciés par des taches bleues et brunes.

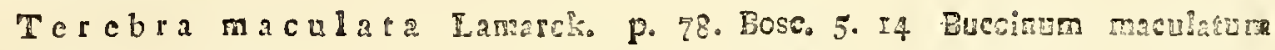
Linnaei. Mattini. 4oto I53. fo I440. Trois indiridus grai présensent quelques différences de couleurs. - Se troure dans la des les lobes ot de l'Afrique

1. 7. Vis favat, fubulée, unie; les tours de fpire (fans fillons intermédiaires?) fans dentelures, avec des taches carrées ferrugineuses.

Tcrebra fubulata. Bosc. Buecinum fubulatum. Lin. Marso 4. E。 I53. fo I44:. Bosc. pl. 32. f. $\sigma_{0}$ - dans la mer des Indes.

8. 9. V a riété de la rnême à tachés confluantes.

IO. II. $V$ is c énelée, Les tours de fpir inférieurement garnis de tubercules.

Tere bra crenulata. Brugụ. Bosc. Buccinam crenulatum Lin. Martini. a. 8. 154 . f. 1445 .

I2. 13. * Vis ja un ise, variété fuperbe, couleur de chair, firies longitudinales blanches.

Te rebra hectic a. Bosc. Chemniez. 11. t. I88. f. 1817. 1818. Buccinum hecticum Linn. - fur les côses de l'Afrique.

14. I5. Vis $\mathrm{d}$ im id i t e, les tours de la fpire unis et partagés par une carène applatie.

Terebra dimidiata, Bosc. Bucsium dimidiarum Lin. Martini. 4. t: r54. f. 1444. dans les mers des Indes et de 1'Afrique. I'un des Individus eft depotsillé.

16 - I8. Vis dup I ic at e, Les tours de fpire firiés, partagés par une large caréne applatie.

Te rebra d uplica la. Bosc. Buccinum duplicntum Linn. Martini. 4. t. I55. f. 1455. - Trois individus présentant quelque diféerence de la couleur. - de Ja mer. des Irodes.

\&. Vis $f \mathrm{trigillé}$, les tours de fpire ftriés obliquement, ot partagés par une carène applatie.

Terebra ftrigillata; - Buccinum Rrigillatum Lin. Martini 4. t. 155. E. r.456. - dans les mers de l'Indes et du. Sibd. 
20. $V$ is mira $n$, presque ftriée; la jonction des tours de fpire doublement crénelée.

Terebra vittata Bosc. - Buccinum rittatum Lin. Martini. 4. t. 155. f. I46r. 1463. 1468. 1469. 1470. - dans les mors de l'Afrique, et de l'Inde.

2I. Vis phallus: les tours de fpire avec des côtes longitudinales; la base de la future inégale; la lèvre proéminente et fupérieurement émarginée.

Terebra phallus Bosc. - Spitzmorchel Martini 4. r. 155. 4464.1465. Buccinum finuatum Born. Mus. caes. p. 264.

22. * Vis ondulée, les tours de fpire ftriés legèrement, les ftries per élevées, ondulées, à l'exreption de la carène applatie, des ftries transverses peu fensibles.

Terebra rndata mihi. Martini. 4. t. 153. f. 1446 . 444 . Bosc. 5. p. $\$ 4$. prend cette vis pour une variété de la zis favat, Terebra fubulata, mais elle en diffère essentiellcment.

23. 24. Vis $t$ ig $r$ in e, Demi transparente, blanche, ponctué de rous: cliaque tour de fpire un peu émarginé fur le dos.

Tezebra tigria a. Bosc. - Buccinum tigrinum Lin. Martini 4. t. I54 f. 1448 . Pays natal inconnu.

25. Vis fuccincte, Aiguë, ftriée horizontalement; les tours de fpire partagés par une feule carène applatie.

Terebra fuccincta Bosc. 5. p. 17. Martini. 4. t. 154. f. 145 J.

25. 2\%. D e ux Individus depouillés qui poroissent appartenir à l'espèce précendente.

28. * V is a $\mathrm{mm}$ irale, alongée, unie, ouverture ovale, les tours, de fpire applatis, ceinturés d'un cordon brun tacheté de blanc.

Terebra ammiralis, mihi. Cette vis eft nouvelle. Elle fe diftingue par fa robe brune d'ombre Ariée de blanc. Les tours de fpire font applatis et ceintures en haut gar un cordon regulierement tachcté de brun et de blanc. Elle a douze :ours et deux pouses quatre lignes de longqeur. I'ignore fon pays natal. 
29. 30. Vis cendréc, Alongée, unie, avec des fascies peu marquées, les tours de fpire entiers à leur future, ftriés longitudinalement.

Terebra cinerea Bosc. 5. 22. - Buccinum cinereum Born. Mus. vindob, Tab. 1o. f. Ir. ז2. - On ignore fon pays natal.

3 I - 32. Vis oblongo-gutté e, alongée, aiguë, frriée longitudinalement, à ftries profondes, le bas des tours de fpire marqué de taches brunes, oblongues.

Tere bra oblongo-gutata; mihi. Born l'a figurée comme appartcmant à Buicinum frigilatumi Lin. mas il l'est trompé. Comparez fa figure (Nus Vindob. Tab. 10. f. 10) avec la déscription de Linnè. - Trois individus de différente grandeur et couleur.

34. 35. Vis a iguille, alongée, unie, très finement itriée en travers; les tours de la fpire contigus.

Terebra acicula Bosc. 5. 29. - Buccinum acicula Lin. Listcr. tab. 1055. f. \%.

36. Vis âpre, Les tours de fpire avec des côtes ftriées transversalement; le premier bossu; le canal faillent.

Terebra aspera, Bosc. Lister tab. 925. f. 17. 18. - On ignore Son pays natal.

37. Vis mu $\mathrm{r}$ ic in e, Reticulée, rngueuse; la fpire recourbée; l'ouverture crénelée; la columelle rugueuse; la lèvre epaisse.

Te rebra muricia a, Bose. Buccinum muricinum Lin. Lister, tab. $9: 6$. f. 19. - On ignore fon pays natal.

38 - 40. Vis tubercul e use, Les tours de fpire aver une fuite de tubercules dans leur milieu.

Terebra tuberculat a. Bosc. - Buccinum suberculatum Lina - Lisa ter. t. 958. f. rr. b. - On ignore fon pays naral.

Espèces fossiles.

4. $T$ is plic at ule, Tirebra plicatula, fubulata: anfractibus plicaris; plicis crebris; inferioribus obsoletis. 
Lamarck. Annal. 2. I66. I. tom. 6. p. 227. PI. 44. E. I3. Lieu natal. Grigeron.

42. Vis fcalarine, $T$. fcalarina Lam. ib. n. 2. MIus, Dem. II. Pa 268. n. 55 .

Tonne. Dolizme. I a marck.

\section{Buccinum Iin.}

Coquille ventrue, fubglobuleuse, cerclée transversalement, à bord droi denté ou crénelé dans toute fa longueur. Ouvcrture obiongue, très ample, Echancrée infériels rement.

Tomier inconnu.

1. 2. Tonne pleure d'oignon, ovale'; les côtes plates.. trois fois plus larges que les fillons; l'ouverture plissée.

Dolium olearium; - Buccinum olearium Lin. Bosc. Dolium Martini. 3. t. II7. f. roz6. Io77. - Des mers des Indes.

3. - 5. Ton ne perdrix, Ovale, oblongue; les cốtes ferrées peu consexe, ondées de blanc; la lèvre droite légèrement ondulée:

Dolium perdix; - Buccinum perdix Lin. Boseo Dolium Martini. 3. to II 7 . fo 1078 . I080.

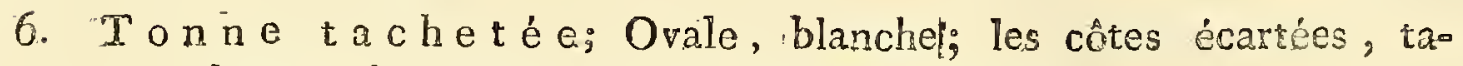
chetées de fauve; la lèvre droite ondulée.

Doli a m maculatum; - Buccinum dolium Lin. Bosc. Dolium Matrini 3. t. II7. fo 1073. - De la mer des Indes.

7. 8. 9. Ton ne fasciée, ovale, garnie de côtes peu élevées; le bord de la lèvre äroite fállant, denté à L'interieur.

Dolium fasciatum. Martini, 3. \&. Erg. f. Ic8s. Buccinum fasciatum Bose. A. p. 265. - De la mer des Indes. Le troisième et be plus grand individu presente un pli singulier de la lève droite qui rend la coquille bossue.

10. - I4. Tonne c a n nelée, Ovale; les côtes convexes dorblées vers le bord fupérieur des tours, intermédiaires plus petites. 
Dokizm gale a Lamarck. - Buccinum galea Lin. Bose. Dolium Mastiai. 3. t. II . f. so6o. - de la ner des. Indss. It yenz des comenglairos de In grénicre grandeur.

\section{Harpe. Harpo Lamarce.}

\section{Buccinum Lin。}

Coq. orale ou bombèe, munie de côtes longitudinales parallècs et tranchaneg. Ouvertur: oblongue, ample, écȟancréc infericureracn ct sans canal. Columelle lisse; *ै base terminé en pointe.

\section{Animal incconnu.}

1. Ha r pe e ntrue; ovale, très coloré; les cótes longitudina les carénées, garnies d"une épine sur les haut des tours; les intersitices striés:

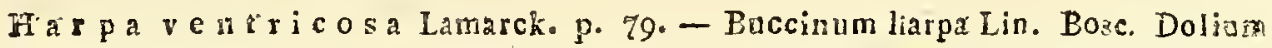
harpa Martini. 3. p. 412. t. rrg. forogo. fq. Vulgairement: la harpe noble. De la mer des Indes. Cet individu est de belle grandeur de 3 pouces ó lignes de longuesr sus 3 pouces 6 lignes de largeus.

- 22. Vingt et un individus de la mênse espèce, présentant de variétés de grandeur et de couleur.

23. Une petite harpe contenant l'hermite Bernards Ciancer bery har. dus I.in. (Pagzurzs Fabrici.)

Espêces fossilus.

4. 25. Harpe mutique, barpa mutica, ovata; costis apica muticis; striis intercostalibus decussatis. $\mathrm{L}$ a $\mathrm{m}$ a $\mathrm{r}$ c $\mathrm{k}$ Anal. d. M. 2. 167 . т. -6. p. $22 \%$. Pl. 44. f. 14.

Casque. Cassider. Brugujè re. Lamarck. Buccinum Iin.

Cog. bombée. Ouverture plus longue que large, terminée à sa base par un canal court; secourté vers le dos de la coqujile. Un boureles au bord drois. Colvmelie plissée inféricuremeas. 
Animal Gafteropode à tête munie de deux tentacules gui portent les yeux à leur base extérieure. Manteau formant, pour la réspiration, un tủbe qui cort par l'échancrure canaliculée de la coquilie. Un opercule cartilagineus attaché au pied de I’animal.

I. - 2. Cas que ba udrier, Ovale, luisant, uni; le bas de la lèvre droite garni de dents épineuses.

C ass idea vibex. Brugu. Lam. Bosc. - Buccinum vibex Lin. Martini z. t. 35. f. 366. - de la mer Mediterranée.

3. Casque hérisson, Ovale, legèrement plissé fur la longueur; la fopire couronnée d'un rang de tubercules; le bas de la lèvre droite garni de dents épineuses.

Cassidea erinace a. B. L. B. - Buccinum erinaceum Lin. Martini 2. t. 36. f. 363. - de la mer des Indras.

4. 5. Casque granuleux, Ovale, marqué de fillons trars. verses et des frries longitudinales; le bas de la levre gauche granuleux.

Cassidea granulos a Bosc. 5. p. 5. - de Ia Mediterranée.

6. ๆ. Variété à taches carrées, qu'il ne faut pas confondre avec le damier, Bucc. areola de $\mathbf{L}$ in $\mathbf{n}$ é.

8. Casque c a nnelé, Ovale, épais, marqué de fillons transverses, lisses; le bas de la lèvre gauche granuleux.

Cassidea sulcosa Bosc. ib. Gualt. t. 39. f. B. - des Antilles. - Ce casque me paruit de même une variété du précédent.

9. 10. Casque pa $v$ é, ovale, lisse, marqué de taches carrées, disposées sur plusieurs rangs; la fpire faillante, garnie de ftries treillissées.

Ca sside a a re ola. Bose. - Bucc. areola Lin. - de la Mediterranée et des Grandes Indes. Favanne. pl. 24. fig。 D. et fig. I.

I I. Cas que pavé, variété à ftries longitudinales fauves. Ma rtini. 2. t. 34. f. 356 . A.

12. I 3. C a sque treillisé, ovale, garni de fries treillisées; les bourrelets de la fpire terminés en dessus par deux dents. 
Cassidea docys sat a Bosc. - Buccinum decsisntum Lin. Martin. a. to 35 fo $260.36 \pi$. 367. 358. - Se trouve dans la Meditorrasue ce dass hes mers de l'Afrigue.

14. * Casque pon c $\mathfrak{e} u \dot{e}$, ovale, garni de ftries transverses peu profondes, treillisées par fept ou huit paires de lignes lono gitudinales ponctuées.

Cassidea punctata mihi. J'en ignote le leu natal.

\$5. * C a sque à $\mathrm{plis,} \mathrm{ovale,} \mathrm{strié} \mathrm{transversalement,} \mathrm{la} \mathrm{lèvre}$ gauche garnie de plis très nombreux.

Cassidea plic a ca mihi. - de la mer des Indas. Aftinis Cassidere ScaBuror Boss. 5. 5 .

16-19. Casque bonnet, ovale, marqué de sillons transverses et de stries longitudinales; l'ouverture oblongue of étroite.

Cassidea tessiculus: - buccinum tosticulus Lis. Martinio 2, so $3 \%$ f. 375. 376. - de la mer des Indies et des Antilles.

20 - 22. Casque $\mathrm{fla}$ a bé, cvale, marqué de plis longitudinaux, tuilés vers le dos, couronnés de tubercules; les satu. res élevées et crénelées.

Cassidea flam me a. Bosc. - Buccinum flammeum. Lin. Martini. 2. 1. 340

f. $353.354 .-$ de la mer des Iniles.

23. 24. Casque tuberculeux, ovale, ondulé de sillons transverses, quatre côtes, et les bords de spire garnis de tubercules obtus.

Cassidea tubcrculata miki. Jignore sa parrie。

25. L e $m$ ê $m$ e depouillé, ne présentant qu’une seule côte tu= berculée.

26. 27. Casque c an nelé, ovale, épais, marqué de sillons transverses, lisses; le bas de la lèvre gauché granuleux.

Cassidea suleosa Posc. 5. 5. Faranne p1. 25. fíg. A. 1. et A. 3. des Antilles. - Affinis Buccino tessiglato Lin. Gu. Martini. XI. p. 750 . I86. t. $1752,93$. 
28. 29. Casque th y r én i e n, ovale, transparent, marqué de côtes transverses; les deux du haut saillantes, tuberculeuses ou plissées; (l'ouverture dentée de chaque côté)?

Cassidea thyrrhena, Bosco - Martini。 Io. to I53. f. I4̨6r. I462. de la mer méditerranée.

30. Casque bézoard, bombé lisse; la spire couronnée d'un rang de tubercules; le bas de la lèvie droite garni de dents. épineuses.

Gassidea gla uca, Bosc, - Buccinum glaucum Lin. Martiaio 2. s. 22. \{. 342.343 .

31. Casque rouge, ovale, rouge, ftrié longitudinalement, le dos garni de plusieurs rangs transverses de gros tubercules arrondis.

Cassidea ru fa. Bosc. - Buccinum rufum Lin, Martini, 2. to 32. fo 34. t. 33. f. $346.34 ?^{\circ}$

32? Casque pom me, ovale, qarni de sillons applatis trans. verses, les deux lèvres denticulées.

Cassidca denticulata. C'est la rême coquille que Marini a décrit

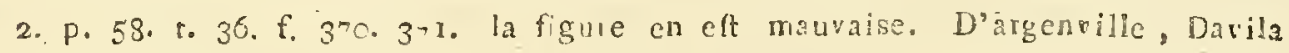
l'out placé parmi les Tonnes, Martini ares Schyrivoet, Hebensreir et Kleira parmi les Semi-Cassides. Je cros qu'elle doit former d'apres les principes de Mr. L a ma r ck un genre particulicr ; a cause de l'ouverture tis étroite, et furtout à cause des plis nombreux et d'un fillon très prefond de la cilumelle, qui donne à la partie inférieure t'ute une autre direction. L inné qui l'a dé irit fous le nom de Buccinum pomun, croit (Mus. Reg. Uiric. p. 600. n. 245.) que ces plis transverses f'obitiven aves l'âge. - Elle vient de la mer des Indes.

Strombe. Strombus. Linné, I a marck.

Eoq. Ventrule, term ée à la base par un canal court, é honcré ou trong é. Bord drni . fe dilatant avec l'âg en aile firm le, entière ou à tin léul tube et ayan méé riburen.ent un tiaus distunce de l'échancrare de sa base.

Animal, incouny. 
I. 2. Strombe ceste, la lèvre saillante antérieurement, arron. die, unie; la spire épinense; la quene obtuse, à trois lobes.

Strombus pugi 1 is Lin. Lam. Boss. Lister t. 863. f. "18. Martini. 3. t. 81. f. 836. 831. - des thers d'Amérique.

$3-7$. Six individus du même, différens en grandeur et en couleur.

8. Strombe grcnouille, la lévre mince, rugueuse, recourbée en dessus; le dos orangé, strié transversalement, cou ronné de verues; l'ouverture blanche, brillante.

Strombus ran iä us Lin. Base. Knorr. Delic. 6. to 29. f. S. - On ignore fon pays natal.

9. Io. De ux variétés du même strombe, l'une rouge plus grande et l'autre plus petite orange.

II. Trois i ème variété à aile plus courte à robe flambée d'orange.

12. Strombe lentigine us, la lévre antérieurement épaisse, trilobée, le dos couronné de verrues; la queue obtuse.

Strombus lentiginosus, Lin. Bosc. Martini. 3. t. 80. 8I. fig. 825 . 828. - Hes mers des Indes et de l'Afrique.

I 3 - 23. O nze individus de la même espèce présentant de différences de grandeur.

24. Var i été de la même espèce, très alongée à lèvre très mince, le dos efi garni de sillons et de très peu de verues.

25. a. b. Strombe coq, la lèvie très-alongée, avec une pointe . en avant; le dos couronné; la queue droite.

Strombus gallus Lin. Bosc. Marcini. 3. 2. 84. fo 84 r. 842. - de 12 mer d'Asie et d'Amérique.

26. St. oreille de $D$ iane, la lèvre avec une pointe en avant; le dos couronné; la queue droite.

Strombus a uris Dianae, Lin. Bosc. Martini. 3. t. 84. f. 938.839. \$4. - des mers d'Asis. 
26 b. St. oreille de Diane, variété à bouche orange et brune. Chem nitz. 10. t. 155 . f. $1487 \cdot 1438$.

27. 28. Strombe sillonné, la lève alongée renflée sur le bord avec une pointe arrondie en avant, le clos cillongé, à sillons élévés dont deux à tubercules, spire alongée, ta. berculée.

strombus fulcatus mihi.,

28. b. c. d. Trois exemplaires du même à lèvre plus mince plissées crénelie.

29. Stro tricorne, la lèvre très alongée, avec une poince plissée en avant, le dos couronné de trois épines.

Strombus ticornis mihi. Vulgairement: le cocu, der alreyeckige braun-roth gefiammte Kampfhahn. Der gehörnte Fechter. Grande aizke de la Famä̈que, Davila Cat. 1yst. p. I83. p. 3 7. Cocblis alata monodactyli: 3 Martini. 3. p. 140. bb. 84. f. 843 . - 845. - tiès rare et fe trouve dans la mes. des Indes.

30. 31. Str. canaris, presque en coeur, la lèvre arrondie, courte, obtuse, unie, ainsi que la spire.

Stro in bus c a na rium, Lin. Bosc. Lister. tb. 853. fo 9. Mastini. 3. t. 79 . f. 817. 818. - Dans les mers d'Asie.

32. Str. canaris, variéte très belle et grande de couleur brunâtre.

$33-35$. Str. e pidrame, la lévre arrondie, courte; le ventre uni; la spire légèrement noduleuse.

Strombus epidromis, Lin. Buss. Lisier. to 853. f. I0. Marto 30 to 990 f. 82 r. - dans les mers d'Asíie.

$36-38 . \mathrm{Str}$. vitté, la lèvre arrondie, unie; le ventre uni la spire alongée; la suture des tours élevée, diftiacte.

Strombus vittatus. Lin. Bosc. Lister. 852. f. 8. Dargenvilie plo g. f. F. Martini 3. to 70: fo 819. 820. 822.823 .

39 - 4I. Str. e ntouré, la lèrre arrondie, obtuse; le ventre uni, avec quaitro fascies pâles, linéairement ponctuéeś. 
Strombus fuccinctus, Lin. Bosc. C'est à cette capèse qu' il faut rapm porter les tigures 815 er 81.6 de Matini 3. to 79. La f̂gure 8I5 a été cmployée

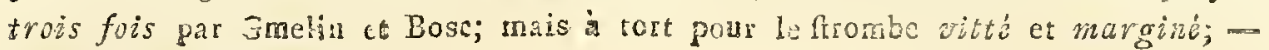
dans la mers d'Asic.

4. 43. Str. cruche, la lère (amincie?), obtuse, courte striée; le ventre et la spire plissés et noueux: l'ouverture à deux lèvres sans épines.

Strombus urceus Lin. Bosc. Rumpho bo 37. f. T. Lister, to 857. fo I 3 . Matini. 3. t. 78. fo 803.806 . the 8c. f. 870 .

4.4. Strombe a i lé, la lèvre antérienement proéminente, arronale, unie; la spire sans pointes, la queue obtuse, à trois lobes.

Strombus a 1 at us Lin. Bosc. Martinio 93. to x. f. 894 ,

45. Strom be marginé, la lèvre saillante; le dos marginé, unie; à queue entière.

Strombus margiatus, Lin. Bosco Chemnitz. ro. to I95. f. I489. 13900

46-50. Strombe bosselé, la lèvre próminente; le dos uni; les tours de spire inégaux, gibbeax.

Stromius gibberulus, Lin. Busi. Dargenville pl. r4. f. N. Martini. 3. i. 87. f. 792. 798. et to 88. f. 863 . Sít. - Se trouve dans la mer d'Asié.

51. 52. Strombe très petit, la lèvre obtuse, bossue; le ventre et la spire avec des plis noueux; l'ouverture d deue lèvies unies.

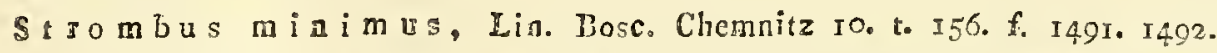

* Strambes à lére moins large n'excédant pas la for* me conique.

53. 54? Strombe fascié, la lève entière; le dos conronné de trois rangs de verrues; entre deux des verrues couleur de rose.

Strombus fasciatus rin. Besc. Martini. ?o P. 87. f. 833 . 834. th. 90. f. 830 t. gr. t. 893. - Se troure da:s les wers d'Afrique.

$55-57$. Strombe lo uhoune, la lève máminente; le dos uni; les tours de spire arrondis, égaux. 
Strombus 1 u hu a u us, Lin. Bosc. Lister. t. 850 . f. 5. Gualt. t. 3r. f. H.

I. Rampl. t. 37. f. 5. Martini. 3. t. 77. f. 789.79 I. 799.

58. Variété du mềme, figurée par Chemnitz. IO. to 15\% f. 1499. 1500. mais la notre a la boucle blanche.

59? Str. de Norvège, oblong, blânc; les tours de spire cylindriques; l'ouverture ovale, évasée; la queue relevée.

Strombus norvegicus. Lin. Gm. Bosc. Chemniz. Io. p. 218. t. 157. f. 149701498 .

60. 6I. Strombe lucifer, la lévre antérieurement arrondie, entière; le ventre doublement strié; la spire couronée de tubercules, les supérieures plus petits.

Stro mbus lucifer, Lia. Bosc. Favanne, pl. 22. f. C. I. Martini 3. to g. f. 878.879 .88 r. 885.886. - Se trouve dans les meis d' Amérique.

$62-65$. Strombe épineux, la lèvre mince en ses bords, entière, un peu plissée, couronnée d'épines très aigües; la spire aigüe.

Strombus fpinosus, mihi.

66. Strombetuberculeux, la lèvre mince en ses bords, entière, un peu plissée, le dos couronné de tubercules arrondis, la spire aigüe, la columelle courbée en haut vers la lèvre droite.

Strombus tuberculatu's mibio

67. Strombe bryone, strombus bryonia, exemplaire imparfait. v. Chemnitz. x. t. I59: f. $1513 .-15150$

Espèces fossiles.

68. Strombe à c a na 1 , Stromibus canalis, turritus, longitudinaliter costatus; basi transverse ftriatus; labro parvo emarginato, in canalem superne decurrentem continuato.

L a ma r ck. Bulletin des $S c$. n. 25. f. 5. Annales du Mus. 2; 22\%. i. J. pl. 45. f. 2. a. b. - Il m'et impossib!e de trouver les motifs qui ont determine M: Lamarck de séunir cette espéce aux nrombes. Diaprès fon propre fystènue elie doit être une rostellaire is canal. - l'ai vu plus tard que-Mr. Bosc en 2 formé arec raison, Ja Rostellaria canciliculata. 


\section{Ptérocere. Pterocera Lam a rck.}

Coq. ventrue, terminée inférieurement par un canal alongé. Bord dioir, fe dilatant aves l’àge en aile diguté, et ayant un fius vers la basi.

Animal inconnu.

I. 2. Ptérocère goutteux, la lèvre à six doigts courbés, la queue recourbée.

Plerocera chiragra; - ftrombus chiragra Lin. Martinio 3. t. 85. f. 853. et t. 87. f. 856 . 1.85\%. Bose. 1V. p. 249. 41. 35. fo 4. - Lians la घeez des Indes.

3. Pterocì re la mbis, la lèvre à sept cornes presque diroites; la gorge unie.

Pre rocera la mbis Lamarck; - frombus lambis Lin. Bosc. Mart. 3. to 8గ. f. 855. dans la mer d'Asie.

4-6. Trois variétés de la même, qui en général vario beaucorp.

7. 8. Pr. Scorpion, la lèvre à sept cornes noueuses, la postérieure très longue.

Pterogera fearpias - - frombus fcorpius Lin. Bosco Mattini. 3. to $\$ 8$. f. 850. - dans la mer des Indes.

9. Variété de la même à cornes lissas.

10. II. Pt. mille pieds, la lèvre à dix cornes droites et courtes; la gorge un peu striée; le dos tuberculé, comprimé. Pierocera millepeda; - frombus millepeda Lill. Bosc. Da:genville pl. 15 f. B. Martini 3. t. 88. f. 86́r. et. 862. - dans la mer des Indes.

I2. P t. pa $1 \mathrm{mée}$, la lèvre palmée en avant, à cornes irès courtes, à bord recourbé vers la gorge, qui efi unie.

pterocera palmata; - ftrombis laciniatus de Chemnitz. 10. p. 223. t. 158. f. 15c6. I507 E-pèce très beile et ues tare, qui est intermédiaire. enrre les ftrombes et les pérocères.

13 - 15. P.t. pied de peli ca n, la lèvre digitée, à quatre cornes très long!nes et alıguleuses.

Pterocera pes pelicani; - Stiombus pes pelicani Lin. Boce. Martina

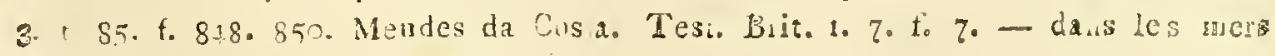
d' Europe, a'Afrique, d'Anérique. 
16. Pt. pied de pelican à digitations plus courtes.

17. Pr. pied de pelican, dont les digitations sont entrelacées avec des serpules, de trois pouces de longeur; - exemplaire très intéressant.

\section{Rostellaire. Rostellaria Lamarck}

Q o g. fửiforme, terminée inférieurement par un canal en bec pointu. Bord droí entier ou denté, plus ou moins dilaté en aile avec l’âge, ei ayant un finus contigu au cacal.

Anirral inconn.

5. Rostella irefuseau, unie, Ie canal alongé et pointu; la lèvre dentée.

Rostellaria fubulata Lamarck. p. 8r. Bosc. 4. p. 243. Lister to 854fo 12. Seba Mus. 3. t. $5^{6}$. f. 2. Favanne pl. 34. to B. 3. Martiaio 4. 2. 158. tol I495 - I497 et t. 159. f. I500- 1502 . - Se trouvc dans la mer rouge. Cet exemplaire eft de la plus grande beauté et a 6 pouces 6 ligues de longueur fur 2. pouces 5 lignes de large avec la lérre dentelé.

2. 3. Deux autres plus délices et plus petites. L'une des deux porte cette escare remarquable dont on trouve la. déscription parmi les escares. V. Tab. VI. f. I.

4. 5. Deux Rostellaires à lèvre non céntće.

Espèces fossiles.

$6-8$. Rostellaire fissurelle, sillonée; la lèvre entière en son inilieu, et se continuant en une fente longitudinale.

Rostellaria fissure11 a Lam. An。d. m. 2. p. 22 r. n. 33 6. P1. 45. Lo 3. Builet. des SG. n. 25. f. 4. - Se troure tossile a Courtagnon. 
Rocker, Maven Isinn. Iam.

Goq. ovale ou oblongre, canaliculéc à $f_{a}$ base, el ayant constamment à l'exiérięr daz bourrelcts longitudirax, perisistans, le plus fouvent taberculeux, épinenx or frangés.

Animal. Garteropode rampant fur un disque ventral muni d' un fotit opercule coné. Tête à deux tentacules pointues, ayant les yeur fitués à lear base cstérie zre. Borche en trompe retractile. Manteau terminé artérieurement par un prolongement ıubuleux.

$$
\text { * Tochers epine us. }
$$

I. 2. Rocher becasse, ovale, mberculé; la quene longue, mince pointue, droite, hériffée de pointes.

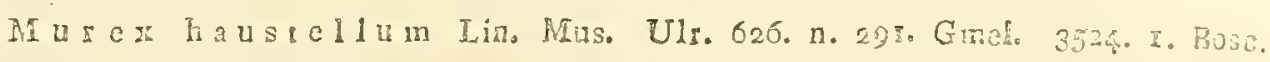

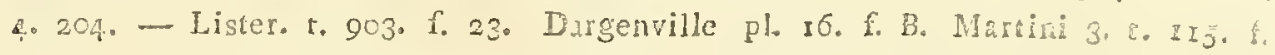
ro66. - Se trouve dans la mer rouge. - Vulgairement ta becrásse.

3. Rocher chause-trape, Ovale, avec trois rangs de longues épines courbes, minces et inégales; la queue alongée, droite, mince, pointue égalemeut épineuse.

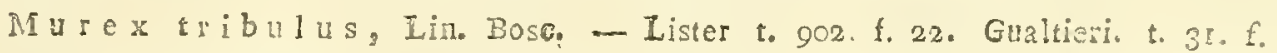
A. 8. 3. 4. Marini 3. \{. 183. א. 1052. - 1056. - Exemplaire de la plus grande beauté, et très bien consẹvé. - Se trouve dáns la mer rouge \&c.

$4-\eta$. Rocher cha use trape, individus plus petits et épines plus courtes,

8-IO. Rochex bulin, presque rond, entouré d'épines min. ces et obliques; la queue alongée, mince droite avec quelques épines.

Murex cornutus, Lin. Bosc. - Matiai. 3. to Is4, f. 1057. - Se trouve

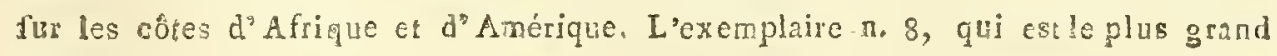
eft garni d'une quartisé de petites Palanes, qui paroissent former une espèce distincte.

11. Exemplaire mutilé de la mếme espèce surmonté de serpules, de Corallines et d'Escares. 
12. I3. Rocher b a nd a ire, presque ovale, entouré d'épines droites; la queue mediocre, mince droite avec quelques épines obliques.

Mures brandaris, Lin. Bosc. - Lister. t. 900. f.' 20. Dargenv. Zoomorph. pl. 4. f. C. Martiai. 3. t. 114. f. 1058. 1059. Bosc. pl. 34. f. 2. Se trouve dans la mer mediteranée. - Le bulin et le brandaire présentent des épines obliques de la queue, ainsi ceci ne peut pas faire le caractère de l'espece mais le deruier, quoique plus penti, a trois feries transuersales d'épiaes far le dos, le bulnn n'ayant que deux, non eomprises les épines de las fpire.

IA. I5. Rochertroncule, ovale, noueus, antérieurement entouré d"épines; la queue courte tronquée, parforée.

Murex tronculus. Lin. Bosc. - Martini 3. t. Icg. fo rors. Iozo. Se trouve dans la médirerranée, et fur la côte d'Amérique.

16. Rocher fascié, renflée, avec une série d'épines blanches fasciées de brun; quatre tours de spire renflés et écartés.

Murex fasciatus. Lin. Bosc. - Knors Del. $\sigma_{0}$ t. 40. f. $\sigma$.

17. 18? V a ríétés de la même espèce.

I9. Rocher cofar, ovale, nodulenx, avec de trois à sept rangs de tubercules; la quene courte et large.

M n rex pom u in Lin. Bosc. - Martini. 3. t. 109. f. I02 I - I025. - Sa trouve fur les côtes d' Afrique.

$20-25$. Six in di vidu s de la même espèce, différens en grandeur et en configurations des fèuilles épineùses.

26. Rocher jaton, ovale, silloné transversalement, avec des côtes converes, en sautoir, et des noeuds perpendiculaires; la queue imperforée, courte.

27. 28. R. méla n a mate, strié transverstlement, avec huit (ou $f(x)$ rangs d'épines creuses et noires; la spire épineuse, et noueuse; la queue mince et pointue.

Murex melanamathos, Lin. Hosc. - Martini. 3. to 128. f. ro:5.

29. * R. a i lé, blanc, strié transversalement avec trois langées de feuilles epineuses, les épines creuses et ailées. 
Murex alatus mihi. Les feuilles, erigées, aussi minces comme du papier, et frangées ou crénelés le lang de la queve, enveloppent du coté droit les épines, de forte que les épines en deviennent ailées. j'ignore fon lieu natal. - Elle ressemble au rocher triptère par la crêce et par ia grandeur, mais elle en diffère par les épines creuses, par fa delicaterse, ou qne je mexpeime ainsi, par fa nature papyracée, et enfin parcequ' elle no se trouve pas fossile. - Il paroit que Mar $\mathrm{M}$ i i a voulu présenter la même coquille. 3. t. III. f. r034. I035. mais la figure n'eft pas reconoissable. La déscription en est un peu plus claire, on y trouve au moins ces épines canaliculées et ailées d"un coté qui rendent cotre coquile si remarquable.

* Pachers r a me uz.

Mulices frond osi, suturis crispato-frondescentibus, cauda abbreuiata.

30. R o cher rameux, avec trois rangs de feuilles; la spire contiguë; la queue tronquée.

Murex a mosus, Lin. Bosco - Aarini 3. ז.102. - I05. f. $980-939$, 995 - 997. - Se troure dans toures les mená.

$3 I-60 . R$ och e r r m e ux, trente individus différens en grandeur et en ramifications de fenilles.

$6 \mathrm{I} \rightarrow 63$. Roc $\mathrm{h}_{\mathrm{e}} \mathrm{r}$ a meux, trois exemplaires à feuilles très épineuses, les épines très longues et courbées.

64 - 66. Rocher scorpion, à quatre rangs de feuilles; la spire en tête; la queue tronquée.

Murex fcorpio, Lin. Bosc. - Rumph. 2. 26. fo 2. Seba 3. t. 77. f. 5. 6. Martini, 3. t. I06. f. 998 . et 1005. L'un eft biun, l'autre blanc, et le troisième eft jeune es presque faus épines. - Se trouve dans la mer de 1'Asie.

6\% - 7o. Rocher des sables, à cinq rangs de feuilles; la spire contiguë la queue courte.

Murex faxatilis, Lin. Bosc. - Martini. 3.t. I0\%. f. I004. I013. 1014. Se trouve dans la Mediterranée et dans la mer des Indes..

21. Rocher, à six rangs de feuilles épineuses; la spire contiguë; la queue longue, recourbée.

Ce $M u$ rex eft de belle grandeur, de 5 pouces ro lignes de longueur, et de 3 pouces de largenr, et paroit former une espéce distincte. 
72. Rocher triangulaire, alongé, triangulaire, presque ailé, réticulé, par des côtes; la queue longue fermée.

Musex triqueter, Borno Mus. vind. Testo t. II.f. r. 2. - Dn igno:e fon pays natal.

* Rocher varique ux.

Murices varicosi, suturis rotundatis torosis cassiscue.

73. 74. Po cher. g y r in, des rangées de gros tubercules inégaux, opposés en grandeur; des groupes de points de tubercules; l'ouverture orbiculaire.

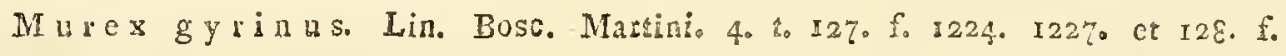
1229. 1235 .

35. 76. Rocher gyrin variété à bandes jaunes et brunes. Martini. 3. t. I 28. f, 1233.1234 .

77 ? Rocher voisin, renflé; des rangées de gros tubercules inégaux opposés en grandeur; la spire pointre, les tour's couronnés, de gros tubercules, l'extrémité seule unie. Murex affinis Lin. Bos:. Valentyin's Schnecken, t. I1. f. 95.

78. Roclier culotte de suisse, de gros tubercules inégaux, presque opposés en grandeur; les tubercules chargés de petites bosses, longitudinales.

Murex 1 ampas, Lin. Bose. - Martiai. 4. 1o r29. Fo 1236. 3233. - Sc troure dans la mer des Indes.

79. R o che r la m pe, de gros tubercules tuberculés, en grand nombre, et presque alternes; le dos pofterieurement uni et strié; l'ouverture sans dents.

Murex Olearium, Lin. Bssc. Guale Test. to 49. f. G. Martini 4. t. I3ิ0。 f. 1242. - Se trouve dans la Médirerranèe et fur les côtes d’Afrique,

80. Rocher cuisse, des rangées, en sautor, de gros tubel cules, trigones, rugueax; les antérieurs noueux; l'ouverture sans dents.

Murex femorale, Lin. Bose. - Lister to o4r. f. $3 \%$. Regenfuss, I. \&. 2. fo 21 Martini 3. t." "ris. f. I039. - Se riquve dans la mer des Indes d'Afrio

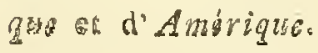


8. Rocher la'vandier, de gros tubercules en sautoir, des noeuds longitudinalement tuberculeux; la queue faisant un angle; l'ouverture dentés.

M u ex lotorius, Lin. Borc. Dargenville. pl, Io. f M. Martini 4. t. r3o. f. 1246. 1277. et. İI. f. 1252. 1253. Bosc. pl.34 f. 3. - des mers d'A Amérique.

82. 83. Ro cher vojet, de gros tubercules en sautoir, un peu noueux, ruggeux; l'ouverture dentée; la queue relevée. M u :ex pi ieare, Lin. Bosc. - Martini 4. t. 130. f. 1242 - I248. - Se trouve dans la n mediterange et fur la côte d'Afrique.

84. 85. Rocher crapaud, sir gros tubercules opposés, alongés, en voute; des cercles de noeudis; la queue oblique. Murex bufonius, Lin. Bosc. - Martini 4. ז. 129. f. I24̧0. I24r.

86. 87. Rocher poire, de gros tubercules ovales, sillonnés transversalement, noueux; la queue alongée, courbée, pointue. Murex pyrum, Lin. Bosc. Martini 3. to II2. f. 1040, 1044, I048. IC5I, - Se tiouve dans la mer des Indes.

88. 89. R oc her à que ue, finement striétransversalement, bur varié de blanc; la queue alongée et pointue; la spire saillante, les tours de spire canaliculés; le premier bossu. M u rex ca udatus, Liq. Bosc. Martini. 3. to II2. fo I045 - Ic4?.

90-92. Rocher-rubécule, de gros tubercules en sautoir. obcus, rugueux, noneux; l'ouverture dentée. Marex rubecula, Lin. Bosc. Nartini, 4. t. 132. f. 1259 et I26\%. - No. 90. et 2 r pr'sentent des variétés brunâtre avee et fans les fascies blanches. Se trouve dans les nuess d'Asie, d'Afrique et d'Amérique.

93. 94. R ocher grimace, gibberx, reticulé par des tubercules inégaur; les gros tubercules er la lèvre dilatés en mem. brane; l'ouverture sineuse; la quene droire.

Mures anus Lin. Bose. Martini. 2. t. 4\%. 403 . 408. - Se troure dans Ia néditerranie. el dans les mer d'Asie.

$95-9 \%$ Rocher réticula iré, de gros tubercules presque opposés, réticulés; des taches tuberculeuses; la columelle presque fans dents; la queue relevée. 
Murez re ticular is, Lin. Bosc. Martini. 2. t. 4r. f. 405. 406. La ciration de la figure $\$ 228$ (some 4. to I228.) employée pour la même espècc chez Gmelin et chez Bose, doit être rayée. Il est vrai que Chaminitz lui-même rapporce cette figure au rocker réticulaire de Linné mais cersanement à cort. * Rochers fans que ue.

Mur ices coaudati fubechinati.

98. 99. Rocher $x$ ic in, Presque ovale; des épines pointues; l'ouverture et la lèvre dentée (dans le jeune age).

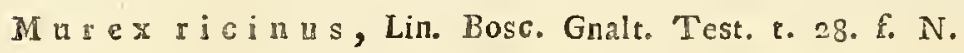

100. Rocher 11 o e d, Presque ovale; des épines coniques, la lèvre dentée; la columelle unie et colorée.

Murex nodus, Lin. Bosc. Knorr Del. 6. k, 24. f. 7 .

IOI I05. Rocher néritoide, Plusieurs rangées de roeuds, la lèvre anguleuse; la columelle un peu applatie.

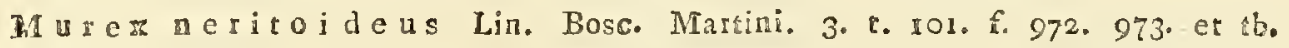
I02. f. $976-9 \% \mathrm{~g}_{0}$ - Se trouve dans la mel des Irdes. - G m e l in p. 3559. a. I69. er aprés lui B os c 4. p. 240. citent encore fuivane Chemniz Io. t. I65. 5. $157 \%$. et 1578. une aurre espéce néritoíle qui pasoit appartenir au gense des Jucins. Ces mêmes ateurs emplojent aussi le rocher Sirat d'Adanson deux fois' l'une fois pour Murex fenegalensis et l'aute fuis pour Marex costatus.

106. Rocher porc épic, Presque ovale, des épines aigües, l'ouverture finueuse.

Murex hystrix, Lin. Bosc. Martiri. 3. t. Ior. f. 974.975.

507. Rocher i mpérial, turriculé, très régulier, à fpires épineuses, les épines creuses, comprimées, furmontées d'une quantité de petites dents regulièrement placées.

M u sex imperialis, mihi. Ce recher est certainement une espèce des plus rares et des plus précieuses. Point d'anteur n'en a fait mention, fa ftructure est auffi délicate que ic meilleur arriste ne pourroit rendre tous les petits Alerails qua il voit. Ce rocher est composé de huit fpires, fillonnécs longitudiaalement ef fumontées aransversalement de feuilles épineuses fuivant toutes les regles de la fymmécrie. La prémiere ligne de chaque fpire qui porte les epines dentelées et furmontées de petites grains est la plus longue et les épines Iont un pea obliques, en inclinant la pointe vers le fommet de la fpire, Ies gusces fons droires et vont toujours en diminuant. 
I08 - Iro. Pocher mélongène, presque orale; les tours de fpire épineux; l'ouverture unie.

Murex melongena Lin. Bosc. Marsini 2. t. 39. et 40. $4.339-39 \%$ Se trouve dans les mers des Indes et de l'Améríque.

II. Pocher manoinelle, ovale, les tours de fpire garni d'épines oblitérées, l'ouverture lisse?

Murex mancine 11 a Lin. Mus. Lud. Ulr. 636. n. 3Is. oú il ajoute "agertura edentula, columella transversim ftriatab", ce qui ne cadre pas avec les figures ciées par Gmelin, p. e. celle de Borr doit être rayée, parceque ce $n^{3}$ est point la mancinelle de Linné. - et ainsi plurieurs autres.

112. Rocher ceramique, ovale, les tours de fpire garni d'épines longues, l’ouverture lisse.

Murex ceramicus Lin. x. 75r. n. 470. Murex capitellun Mus. Reg. Uli. p. 634. n. 286. Voluta ceramica ed. XII. Miarini. 3. to 99. fo. 94: - des cors de Coromandel.

11. Rocher a $\mathrm{r}$ é, ovale, les tours de fpire armés d'épine: arrondies très fortes, l' ouverture lisse.

Murex armigerus mihi. Buccinum armigerum, Chemnitz. 11. t. 187. \%. I 798. 1799. p. 82. 83. On ignore le pays d'où il vient.

114. 115. R ocher à tro is côtes, tringulaire à trois fillons Ionjitudimax très gros, ftriés transversalement, la lèvre droite crenelée.

M urex tricostatus mihi. Il appartient plûtôt $\dot{a}$ la division précendente, et doit occuper fa place à coté du Murex alatus et triqueter, quoiqu'il est beacoup plus giand et très pesant. Voici pourquoi Martini l'a appellé Purpura triquetra poiderosa. 3. p. $347^{\circ}$ t. 110. fo 1029. 1030. - de la côte de Coromundel.

I6. Rocher plissé, ovale, les tours de fpire plissés et noueur.

Murex plicatus Lin. Bose. Martini. 4. t. I23. E. I I4: II 4 2. - Se trouve dans la mer des Indes. 
II\%. I8. Rocher mure, ovale, les tours de fpire garni de tubercules granuleux, I'ouverture dentée, violette. Mirex m oxum. - Marini 3. t. 10r. f. 970, 971 . Se trouve dans les mers des Irdes.

II9. I:O. Rocher tonneau, ovale, ouvert, à queue, les tours de fpire avec quelques cercles élevés, arondis fubcanaliculés.

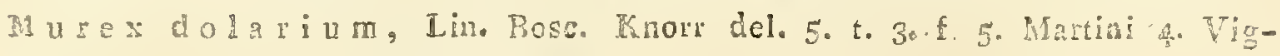
tretre. A1. - Se srouve dans l'Océan.

E2I. I22. Rocher c abestan, ovale, ourert, les tours de pire avec quelques cercles élevés, obtus.

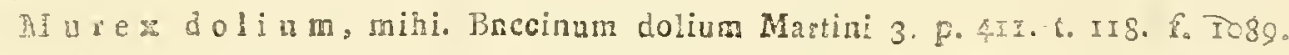

123. I24. Rocher treillisé, ovale, folide, opague, les tours de Spire treillissés, un fillon distinct.

Murex cancellatus. Lin. Boss. Knorr Del. 2. t. $2 \%$ f. 3.

I25- - 129. R ocher mage 11 a nique, ventru, ombiliqué, frié transversalement, les tours de fpire avec des côtes parallẻles, le premier très grand.

Muғex magellanicus, Lin. Bosc. Martini 4. to I39. for $29 \%$ Kran De1. 4. to 30. f. 2. - - Se trouve an détroit de Magellan.

I30. Rocher variqueux, ovale, à fillons longitudinaux, firié transversalement, la quene courte.

M ksex varicosus chemnitz. I0. p. 256. t. I62. f. 1545. 1547. - du cap de bonne Espérance.

131. R ocher nasse, folide, noir, ou d'un brun clair, avec une fascie blanche presque diaphane; les tours de fpire nou. eus: la columelle un peu plissée.

Murex nassa. Lin. Bosc. Martini. 4. t. I22. f. I131- I I34. * Rocher, c a udigè res,

C a u d ig exi, cauda fubulata ex parte. :

Jora. Certe fousdivision de Linné-Gmelin doit être entiérement refondue, vu que la plus grande partie des espè ces entrent dans les genres, fuseau et pyrule de Lamarck et que d'autres p. e. celles qui fuivent peuvent être placées parmi l'une ou l'autre des précéndentes. 
532. Rocher couleny de chair, solide, ventrue, hes spires garnies d'épines coniques, le dos ayant quatre ou plusieurs tubercules épineux.

Marex carnaries Chemnitz. 10, 270 . 4. 164. F. I556.

I557. Ges cotes de Tranquebr. Buccinum ampullaceum I.ister. \%. $88 \%$. $6 . \mathrm{b}$.

33. 139. $\mathrm{Roch}$ or o h a $\mathrm{ve}=\mathrm{s} 0 \mathrm{y}$ is, solide, ventrue, les spires garnies d'épires comprimées, lo dos uni.

Murex vespertilio Iin. Born. Martini. 4. t. 142. f. 1323.

5324.1326. 132\%. 162. Murex pugilinus Born Index

p. 314

I35. Rocher cochlidie, alongé, à queue; les tours de spire appiatis an dessus.

Hurex cochlidium. In. Bose. Chemnitz. 10. t. I6q. fo I56g.Se trone dans l' Cósan Indien.

【36. Rocher trapeze, alongé, obtusément anguleux; les tours de spire un pen noveux; l'ouverture dentée; la queue courte ct circite.

A urox trapezillin Liso. Bose. - Martini 4. 4. 140. f. E3ro. I3rto - Se touve dans la incr diss Intás.

3\% Rocher corné, oblong, inégal; les tours de spire enve. ioppes à leur base; le bout tuberculeux, l'ouverture sans dentes; la quene rélevée.

his rex cortacus Lin. Gmel. Bosc. Gualt. to 46, f. F. - dans la mer du Nort.

138. Pocher åpre, plissé longitudinalement, avec des côtes transverses; la spice un peu alongée, l'ouverture ovale, la lerre crenclée.

in u i $x$ asper Lin. Gmel. Bose. Mastini 4. 'to 144. fo I3t2. - Sc trouve dans la mer des Indes

139. Rocher antique, oblong, onvert, à cqueue, arec huit tours de spire cylindriques.

Mu rex antiqu cus Lia. Fa. fuec. 2165 . Bosc, - Martini 4. t. 338 . f. 1292 . da24. 1296. Se rroule dans les mers du Nord. 
140. 14:. Rocher trompette, ventru, oblong, uni; les tours de spire arrondis; l'ouverture dentée; la queue courte.

Murex tritonis, Lin. Bosc. Martini 4. t. 13!. f. 2277 . t. I35. f. I283. t. I 3ró. f. r 284. 1285. - dans les mess d’Asie, Ia mediterranée, et fur les côics d' Afrique.

is pèces fósiles.

142 - 146. Rocher triptère, Murex tripterns, trigonus, .elongatus transversim sulcatus; angulis alato-membranaceis.

Lamarck Anal. d. Mus. 2. 222. I; 6. P1. 45. f. Aุ.

147. Rocher tricariné, mirex tricarinatus, ovato-oblongus, tricarinatus, transversim sulcatus, angulis frondosis difinctis, subspinosis. Lamarck. ib. n. 2.

149. Pocher en cha hse-trape, murex calcitrapa. Lam. ib..n. 4 .

149. Rocher grillé, mavex clatbratus. Lam. ib. p. 224. n. $\%$

150. Rocher ftriatule, murew ftriatulus Lam. ib. p. 225.

ก. 9. 6. Pl. 45. f. 5

15i. Rocher nodulaire, murex nodularizs, Lam.ib. n. I5.

152. Rocher tubifèe, marcx tubifer, Iam. ib. I I

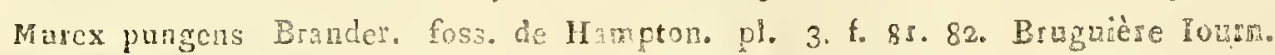
d' hist. 17. n. 1. p. 28. plo 2. f. 3. 4. Lieu natal. Grignors.

Fusean. Frisus. Brugu. Lam。

Cuq. fubfusiforme, canaliculée à fa base, ventru dans sa partie moyenne ou infericurement, ayant ha fpize nlongée ct depourve de bourrelers persistants à l'extéricur. Columelle lisse; bord droir fans échancruse.

Animal' inconu.

1. 3. Fus e a u à $\operatorname{long}$ u e que ue, turriculé, caudé, presque droit, ftrié, noueuz, caréné.

Fusus longicaud atus Lamarck. po 82. Fusus Rumpho to 29. f. Fo Lis-

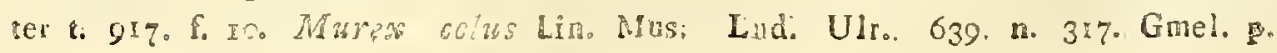


3543. ก. 65. Bosc. 4. 221 . Favanne pl. 33. f. A. 5. 35. f. C. I. Martini. A. E.

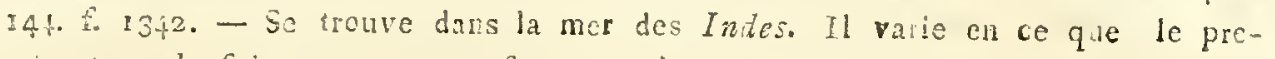
miar tou: de fpire cst anec ou fans noeuds.

4. Le fuseau blanc, fusus candidus, murex candidus de Gmel. 3556. n. 1 I3. et Bosc. 4. 234. d'après Chemnitz. 4. t. 144. f. I339. - n’est qu'une varićté du précédent.

5. Fuseau a u a n, turriculé, caudé, les spires nonenses, l'onverture anguleuse.

Fusus arua a us m. - Murex aruanus. Lin. Gm. Bose. - Chemilz. A vign. 39. f. D. - Se kroure dans les mers d'Asie.

6. 7. Fusea u $\mathrm{n}$ iva $\mathbf{m}$, brun, avec des fascies jaunes les cours de spires noueux, l'ouverture fubanguleuse.

Fusus Morio, m. Murex morio Lin. Bosc. Chemaiz 4. t. 139. f. !300. I30I. - So trolave fur les córes d'Afrique.

8. Fus eau n.ifat, ventru, uni, la spire ftriée, les tours de spire arrondis; l'ouverture unie, la queue courte.

Fusus pusio. - Mirex pusio Gmel. Eos:. Chemeirz 4. to I47. 1. 1357.- Se trouve dans la méditerranèe fur la côte d'Amérique.

9. Fuseau perron, presque turriculé; les tours de spire carénés en délessus, marginés, applatis, la queue droite, alonģée.

Fusus perzon; - Murex perron Gmel. Bosc. Chemn. Io. 6. I64. f. 1573.Se trouve dans la mer du 3ud.

Es pèces fossiles.

10. Fusean ridé, fusus rugosus Lam. An. 2. 316. 1. 6 . P1. 46.1 .

Murex porrectus Brand. Foss. p. 2I. t. 2 fo 35 - - de Grignon.

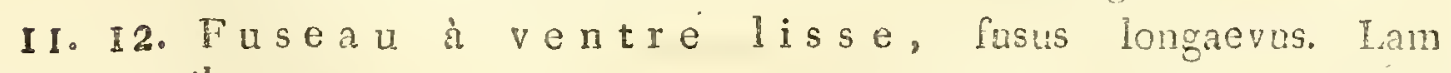
ib. n. 3. .

Murex longaevus Brand. p. 22. f. 40. 73. et 93. M. herigutus Gme1. F. 3555. n. 6 - de Grignon.

13. I4. Fusea u 1 ubulé, F. subulatas Iam. ib. 0. - de. Grignon. 
15. Fascan tortile, fusus intortus; Lam. ib. n. 8. de Grignon.

16 -24. Neuf Iusear polygones, $\mathrm{H}$. polygonus ib. 319. n. 9. de Grignon.

\section{Pyхule. Pyrula. $\mathbb{L}$ a ma rek。}

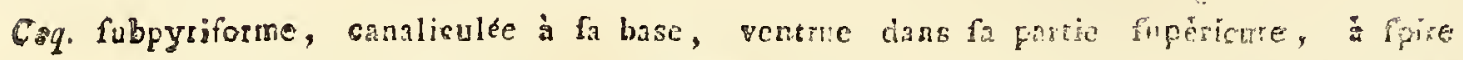

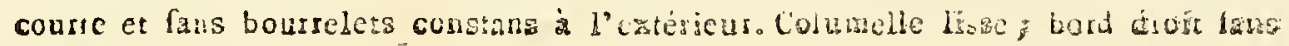
échascrure.

Animal inconau.

1-6. Pyrule figue, onverte, à queue friés les tours de atre avec un canal difince.

P у г 1 a fícus. Lam. p. 32. Musex et Bulla ficue Litro

7- I2. P y r l e $\mathrm{r}$ a ve, solide, ombihquée, firiée transwersalement à trois rangs de noeuds, l'ouverture ample ftriée.

P yrula rapa; - Mures ripa Lin. Busc. Markini. 3. t. 53. f. $750-753$.

33-15. "P y r u le unique, ouverte, sinué, la spire cournés à gauclio, conronnée a’épines pen apparentes.

pyrula perversa:-- Murex pertesu= Lin Bosco Borno t. ir. f. \&. a.

15-10. Pyrule canaliculée, ouverte, les toms de spiro avec un canal difinct.

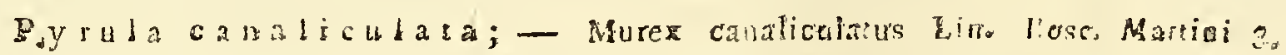

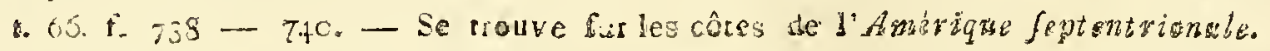
Espocs tossiles.

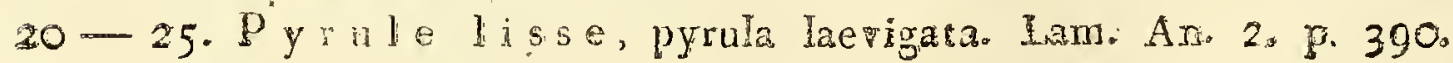
n. $1 ;-6 . p^{l} \cdot 40.7-$

Fasciotaire, Fusciolaria Lamatek

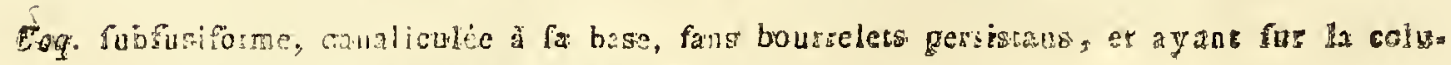

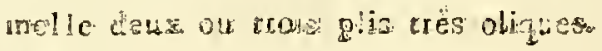

Amsiasat, incon:anm 
I- IA asciola ire talipe, ventrue, oblongue, anie: les cours de spire arrondis; la suture double; la queue ouver. te frriée.

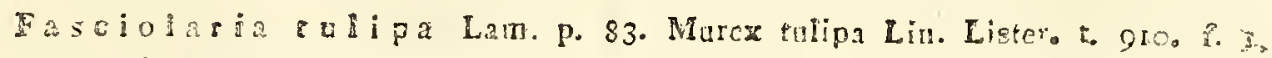

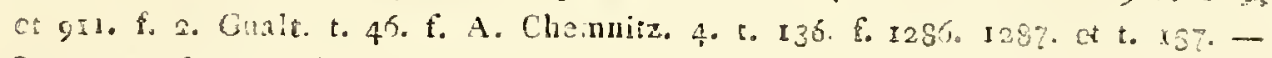
Se sroure fus les côtes d'Amérique. Elle varis bazuconp do carbeur or de

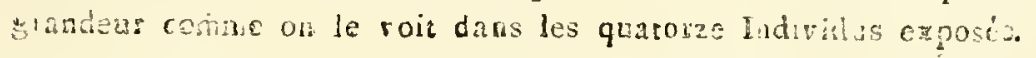

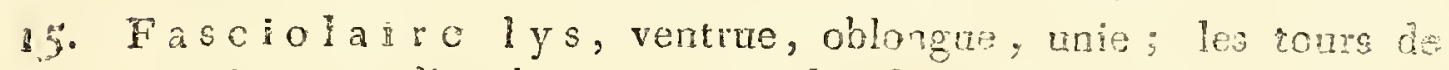
spire arronlis; la suture simple, la queve courte et lisse. Fasciolaria li itum, milli; clle est blanche, ob courcte de lignes earas-

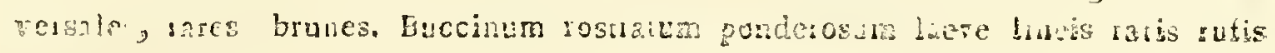
chediadutumo L.ster to 210.

\section{Tarbine l le. Turbinellus $L$ a in arck.}

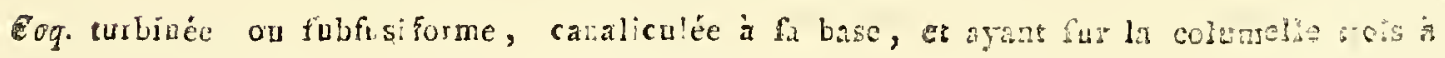
cing plis compr més et transucrsez.

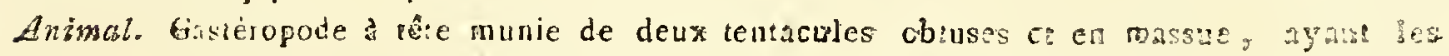

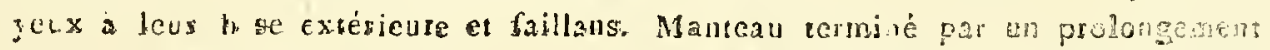

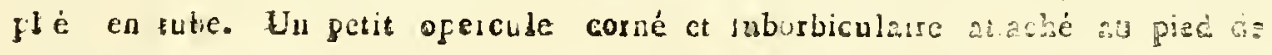
ranimaI。

2. 2. Turbinelle épine use, zurriculée, les tours de spire garnis d'épines très fortes, Ia columelle à ciuq piỏs, les intermédiaires plus petites.

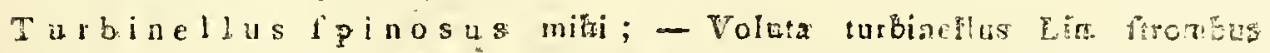

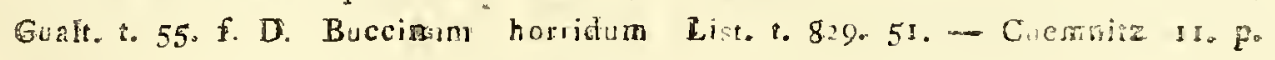

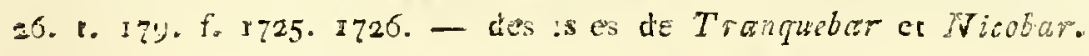

3. 4. Tarbinelíe couron ne; turriculét, les tours de spire garni $\mathrm{d}^{x}$ une rangèe d’épines et de plusieurs leuiltes tranchano tes, la columelle a trois plis.

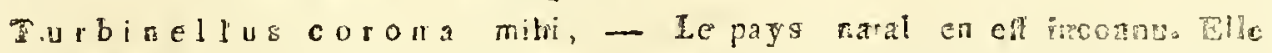

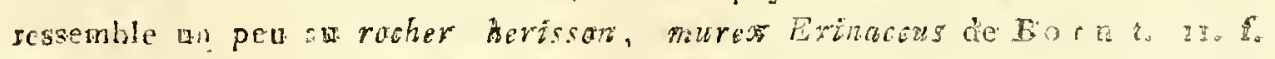

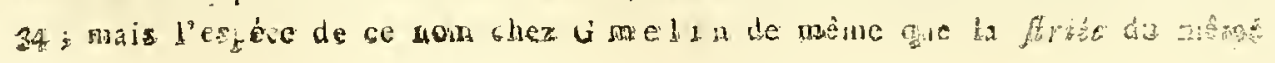




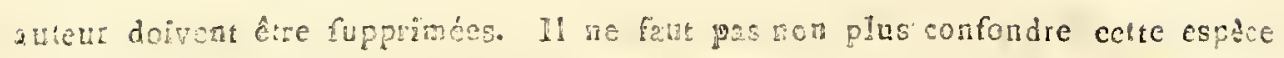

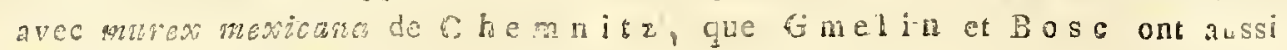
appellé corosa. C'efo un erai mureñ.

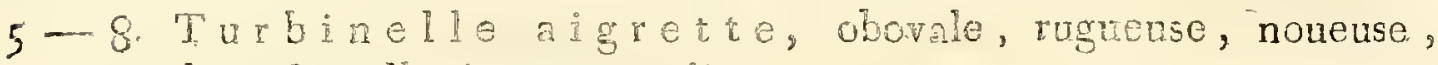
la columelie à quatre plis.

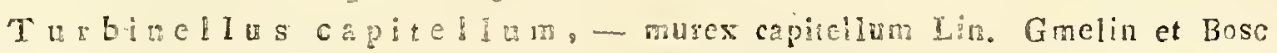
ont omis cotte espèce, qui cependant ef bien distinote. Linné en comptant quatre plis de la columelle a omis la prenière élevation, qui en vétité ne peut pas êle régarde comme Ln Pli. - Mnatini. 3. t. 99. f. 947.948.

9. Turbinelle chicorée, ventrue, ftrée transversalement; la spire obtuse, et onduleusement noduleuse, la columelle à quatre plis.

Turbinellus fcolymus; - Murex fcolymu Gnel. Bosc. Chamnitz 4. t. 142. f. I325. Elle eft transparente rans être mincc. Son pays natal eft illónnu. C'eft une des plus rares coginilies.

I. Turbinelle entonnoir, fusiforme, noueuse onduleusement, sillonée transversalement; la columelle à trois plis.

Turbinellus infundibulum; - Nures iufundibulum Gmel. Bosc. Chemaitz. 4. Vign. 35. f. A.

I $\angle$ I 3. T arbinel le polygone, un peu ventrue, avec cies tubercules onduleux; friée et sillonée par beancoup d’angles obtus; la columelle à trois plis.

Turbinellus polygou us; - Murcs palygmus Gmei. Bosc. Chemnitz. 4. 6. 170. f. I306. I300. - Se trouve dans la mer des Indes.

14. Turbine $1 \mathrm{l}$ e $\mathrm{l}$ ig n e us e, subturricnlée, fitriée et sillonnée transversalement, la queue courte, ombiliquée, la columelle à deux plis oblitérés, la lèvre droite, unie.

Turbinellus lignarius, mihi. - Murex lignarius Gmel. Bose. Knorr Del. 6. t. 26 . f. 5 .

15. * Tu r bi nelle fla m bé e, subturriculée, les tours de spire peu diftincts et large, sillonnée longitudinalement, et itriées transversalement, la queue courte ombiliquée, la columelie à quatre plis, la lèvré crénelée. 
Turbinellus flammous mihi. Elle est blanche et los fillons longitunaux jauné d'orange. J'ignore fon pays natal.

16. Turbinelle vill e, turriculée, à sillons longitudinatz ét à côtes transversales, la queue courte, la columelle ì deux plis.

Turbinellus fenticosus; - Murex fentieosus Lin. I2. n. 54 f. II20. Gmel. 3535. n. 49. Born. p. 306. Index pag. 304 Distelfchnecke; - Mutux cancellatus Karsien Mis. Lesk. I. F. 266. n. I020. f. 6. Chemnitz. IR. p. I3z. 1. 193. f. 1864 -- 1866. - des la mer des Inades. - Cetre dernère especo paroit appartenir a a Clavatules de Lamarck fuivant fa forme totale. Mais lez plis de la collumale, ci le defaut du finus au bord droit, la sendent espete ingermediaire entre ieș deux genres. .

\section{Pleurotom e. Pleurotoma $\mathrm{I}$ a $\mathrm{m}$.}

Coq. fusiforme, ayant louverture terminée inféricurement par un canal alongé. Une entaille ou une échancure au bord droit près de fon fommet.

Aramal. Gestéropode rampant fur un dişąe alongé et élcvé an dessus de ce disque fur un pedicule cours, épais ct cylindrique. Têre à deux tentacules pointus ayant les yeux à leur base exiérieuze. Manicau debordant fur les côcs, et terminé antérieurement par un prolongemeat plée en tube. Un petit opersule corné attaché au picd ou disq̨ue de l'animal. A r genvill e Zoom, t. 4. f. B.

1-5. Pleurotome babylonien, fusiforme, à queue iongue legèrement sillonnée, l'échancrure longue et parallèle. plezsoroma babyloaica, Lamack p. 84. - Musex babylonius Lin. Eist, t. grz. t. 11. Chemnitz. 4. t. 34.3. f. 1331. 1332.

6 - 3. * Ple nrotome sillonnée, fusiforme, à queue longue, fortement sillonnée, l'èchancrure courte et conique. Plecrotoma fulsata mihi. l'ignore fon licu natal.. .

\section{1 a va tu. I e: Clavatula. La m.}

\section{Murex Lin.}

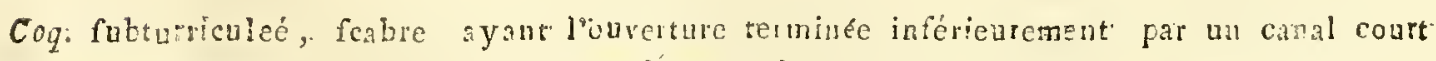
ou par une échancrure: Un fínts an bord droit piès de foul fonmet.

Animal iticomu. 


\section{IOE CLAVATULA. CERITIIUA.}

A yes striés, granulenx.

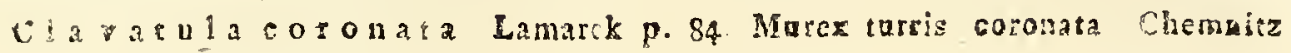
r.t. 590. to 1831.2832 .

\section{$C$ I it e. Cerithints.}

Adanson. Bruguière Iamarck. Marest In.

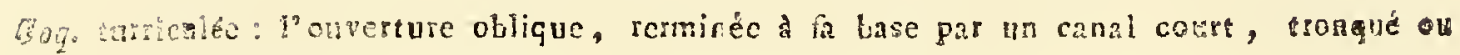
zecourbé. Unc gouttière à l'extrénité fupétieure du bord droit.

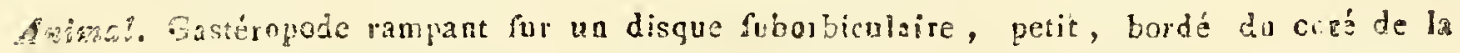

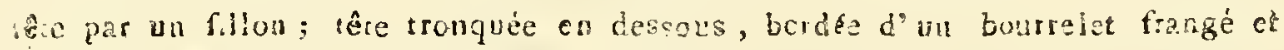
munie de deux tcncacules aigues, ayan les yeux près de luto base externe. Un pesit opercale orliculaire et corné attaché au pied de l'anumal.

* Ceriles a canal ruès recourbé.

1. Cérite obelis que, varié de brun; les tours de spire garnis de quatre côtes granuleuses; la columalle marquée d'un pli. Cerithill mobeison Bruguierc. Eosc. 4. 180. Pl. 29. F. 5. La citation

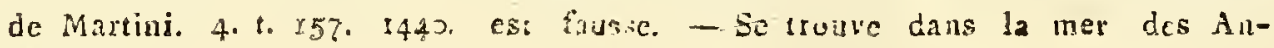
tilics.

2. 3. Céxite b u irc, ventra, lisse, blanc; le bord supérieur des tours de la spire plissé, la colmmelie marquée d’un pli.

Ccrithiusn vertagus Bace. - Murex verragus Lia. - Chenaitz. 4. to 156. f. 54 ?9 - Se trouve dars ia mes des indes.

4. 5. Cérite raboteux, blane; les tours de la spire profondement plissés, et fíniés transversalement; chaque pli garni de trois tubercules; la colunelle marquée d'un pli.

Cerithium asperam Bcso. - Murex asper Lia. - Chemate 4. to 55. 1. 2483. -- dans la wer des Antilles.

6. 7. Cérte fascié, presque cylindrique; les tours de la spire plissís longitudinalement, garnis de trois stries transverses, et de trois bandes jaunts; la columelle marquée d'un pli. 
Cerithium fasciatum Busc. Murex fasciatus Grot. Chemnitz. 4. t. 157. f. 1481. 1482. - dans 12 mer des Indes.

8. Cérite granuleux, étroit, fauve, les iours de spire garnis de stries transverses, et de trois rangs de tubercules grenus; la columelle marquée d'un pli.

Cerithium granulatum, Bosc. Murex granulatus Gme1, Chemnitz. 4. 8. 15\% f. I492. - dans l'océza asiatique.

9-II. Cérite chenille, tachelé de brun; les tours inférieurs garnis vers le milicu d'une côte tuberculeuse, ceux du haut de la spire striés transversalement.

Cerithium aluco Bosc. - Murer Aluco Lin. - Chemnitz 4. to ${ }_{5} 6$.

f. r478, dans la mer des indes

I2. I 3. Cérit a varié, blanc, tacheté de brun ou de rouge, les tours garnis au milieu d'épines, l'ouverture lisse.

Cerithiu va viegatu in mihi. - le bec du cosbeau Knorr Deí. ?o to I6. Fo 5. - La parrie en eft inconnue. No. 13. paroir une espèce distinc:e.

- Cé si tes a bor ds du canal légèrement recourbés.

I4 - 16. Cér i te massue, epais, jaunâtre, tacheté de brun, les tours de la spire plissés, striés transversalement et nodulenx; le dedans de la lèvre droite silloné.

Cerithiu m ciava, Bosc. Murex Clava. Gmelin. Chemnitz Io. p. $250^{\circ}$ Vigo. 22. f A B. - dans la mer des grances Intes.

17. Cérite noirci, variqneux, noir; les tours de la spire garnis de striés transverses, et de deux côtes tubercnleuses, la plus grande située au inilieu de chaque tour, la seconde près de leur bord supérieur.

C crithium atrat um Bojc. Murex atratus Borntest. to Ifof. 1 \% 18. Cans la mer des Aintilles.

18. Célite latissoire, brun, les tours de la spire garnis de quatre à cinq côtes tuberculeuses; les tubercules de la seconde côte du coté de la spire, plus gros que ceux des auties.

Ceritbium radula, Bosc. 4. 538. P1. 33. f. 1. 2.3. - Ihusex radila

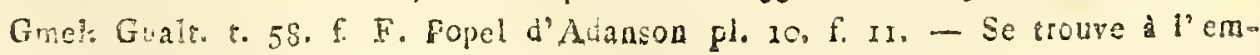
bouchure des sivières d'sfrique. 
I9. Cérite ly exagone, hexagone, jaunâtre; les tours de la spire garnis - de trois côtes granuleuses; la côte supérieure du tour de l'ouverture fortement tuberculeuse; la lèvre droite épaisse.

Cerithium hexagonum, Bosc. - Murex hexagonus Gmel。 Chomnitz. IC. t. I62. f. 1554. 1555. - dans la mer du Sud; - et fossile à Courtagnon. Espèces fossiles.

20. Cérite échinoide, Cerithiun echinoides, asperum; anfiactum costis binis trinisve transversis tubcrculato -imuricatis, insequaibus.

Lam. Aral. du M. 3. p. 273. n. To Lieu natal. Grignon.

2.5. Cyérite anguleux, Cerithium angulosum, pyramiatum; transrerse ftriatum; anfractibus nedois carinatis angulatis; canali brevissimo.

Lamark. ib. B. B. Lin. Grignon.

\section{B. Cuverture entière et sans canal ì sa base.}

Obs. Dans les genres de cette division le manteau de l'animal ne forme ausur prolongement tubuleux pour la resjiration.

$$
\text { Tou p i e. Trocbus. } \mathrm{L} \text { i } \mathrm{n} \text {. }
$$

toq conique. Louversure presque quadrangulaire, déprimée transversalement. Axe oblique fur le plan de la base.

Animal. V. Lam. p. 85 .

3. Toupie nilotique, conique, unie, un peu ombiliquée. Trochus nil oricus. Lin. Bo:c. Lister, to Gr\%. f. 3 . Chemnizz. 5 to $56 \%$ fo i605. r609. - de la mer des Indes.

2-4. Toupie maculé e.

Trochus maculatus, Lin. Bosco Chemnitzo 5. to r 72 , f. 169r. róg.

5. 6. Toupie parsemée.

Trochis coaspersus Lin. Bosc. Chemnitzo 5. r. 369. f. r62\%. - Se trouve dans la mer des Indes.

7- I 3. T T u pie sor c i è re, sept individus de diff́crente grandeur. Trochis magus, Lia, Bose. Chemnitz. 5. to 1 g. 1. 1656. 1600. - Se trouve su: les côtes d'Afrique. 
14. Toupie applatie.

Trochus depressus, Lin. Bosc. Chemnitz. 5. to 1qt. I. IGS8. et $\pm 66 g$.

I5 - 18. Toupie vallet, quatre individus.

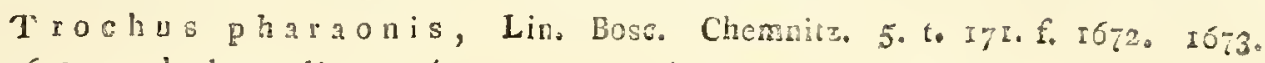
r678. - de la mecuiterranie.

19. Toupie concave.

Trochus Goncavis, Gme'. 357. r. 21. Bosco itop. 154. Chemnitz. 5. t. I68. f. I620. I6ai, - dans la mer des Indes.

20. Toupie ombilicaire.

Trochus umbilicaris, Gmę. p. 3568, no ta. Eosco Chemaitz. 5. I73. f. 1666. - dans la Médríterranée.

$2 \mathrm{I}$ - 23. To u p i m odule, trois exemplaires.

Trochus modulus, Gmel. 35?8. n. 8. Bosc. 4. I50. Chemite go to Ig. t. 1665. - Se irouve dans la mer routge.

* Toupies imperfores.

24. Toupie obelisque, conique entourée d' un grand nom. bre de séries de grains blancs ou verts, columelle à une scule dent.

Trochus obeliscus, Gmel. 3579. Bosc. 5. po r68. Chemnitz. 5. to I60, f. $1510-1512 .-$ Se trouve dans la iner des Inctes.

25. Toupie couleurdeciel.

Trochus caelatus Gmel. 358ron. 95 . Bose. 3. rin. Knorr Del. 5. t. I2. f. 3. (hernn. 5. t. I62. f. I53\% 1537. - Se trouve fur les côtes de d'A mérique méritionale.

26. Toupie truffe.

Trochus tuber Gmel. 3578. n. 77. Bosc. 4. I67. Chemnitr. 5. t. I64. f. 156r. et 556. f. 1572 - 1576. - daus la Méditerranée et sur la côte d' Anérique.

27. Toupie fovéolate.

Trochus fove olatus. Gmel. Bosc. Chemnitz. S. ?. 16t. f. $1516-1519$. Se trouve dans la mer rouge.

28. Toupie i mbriquée, pyramidale, avec des côtes, des sillons et des plis longitudinaux; les tours de spire élevés en leurs bords. 
Trochus imbricatus, Gnel. Base. Chemniz 5. 5. т62. f. 153ז.I533. - - :e noure daus la mor d'Anérique.

29. Toupie inerne.

Trochus inenuis, Gmel. 3575. 62. Bosc. 4. 162. Chennitz. 5. t. I 73 . f. 1712.17130

30. 31. Toup i e petit cône, conique, unie; les tours de spire aver une ligne élevée, les derniers tours élevés.

Tro.hus conulus, Gnelia. Bosc. 4. I58. Chemuiz. 5. t. I65. fo r583. I59r.

32. Toup ie grainue, conique, les tons de spire ondulés avec des tubercules et des cercles grainus.

Troehus glanulatus, mihi. Chemnirz. 5. t. I68. f. 1619. des cô.es de la Chine。

33. Toupie noduleuse.

Troch us nodulos us, Gmel. Bosc. Chemnitz. 5. t. I'3. F. I545. 1546.dans la mer dú Sud, et sur la cốc de l'Amérique merítionale.

34. 35. T o p ie chin o ise, obtusement pyramidale, noire, la base fasciée de pourpre; la columelle blanche; tous les tours de spire arrondis.

Troehus sinensis. Gmel. Bosc. Chemnirz. 5. 2. 165. t. I564. I56g.

36. 37. Toupic rostrate, pyramidale, transversalement striée, variée de blanc et de rouge; l'extrémité demi-transparente et verte.

Trochus rostratus Gmelin. Bosc. Chemnitz. 5. to I6I. f. 1524: I525.Sc trouve dans la mer du $S u t$.

38. 39. T o u p i e i r idée, très unie, hleuâtre, variée de lignes rouges, ondulées et anguleuses, et des ftries iridées.

Trochus Iris, Ginel. 3580. 86. Bssc. 4. I69. Chemnitz. 5. t. I6I. f. 1522.1523 .

I. es espèces fos s il es sont décrites vol. Ir. p. $270^{\circ}$

* Toupies turriculées.

Toupie porte coquille, les tours de spire cylindriques, un pəu plissés, le premer et l'ouverture applatis. 


\section{$\begin{array}{llllllll}X & E & N & O & P & H & K & \text { A. }\end{array}$}

Obs. La coguille qui cst connue sous le rom de frippiè ze ct lont nous onsséduis de supcrbes échantillons, dnit former un genre parteclier à ce qu'il me semble, le quel se fait vloir par les caractécs suivars: Co quilic subturrieulée, átours despire fermés pardescorps étrangers; l'oucrturelisse, cnorcille, la columelíe applatie, donnant desriérelebourrelet originc ides côtes élcrécs qui aboutissent aucontour de la prémicrespi1c; (presque to jours auprès du conps sirange: que la coquille a soudé). Il faut que l'animai ais une communication cutre les spircs avec l'cxtéricur pou faire suinter ce liquide qui amache les corps étrangers, et affermit les coure. L'animal parot faire ce procédé avec choix, car on rerra toujours les petis: co-ps vers le sommet et de plús gres vers la base, lesquels sont placés ainsi, que 13. base de la coquille ne touche par la terre. Ces obfervations doivent persuader les sauralistes d'établir un genre particulier des foit-disans fripières, pour laquel je propose le nom: Xenophosa, coquilie partant des corrs étrangers. L'animal csi inconnu, mais doit présentez beaucoup d'interêt à l'anatomifte ct au physiologifte. 11 peut même devcnir intéres ant pour le géognofte, en liz transportant des cailloux des fonds des ricrs, que son regard ne peut pas arcináre. - Les Xenoplores se trouvent dans l' acéan Américain.

I. * Xenopliore 1 i s se, les tours de spire comprimés, lia base lisse, les côtes de l'ombilio visible à l'origine.

X c noplora lacvigas a mihi. Elle présente dans sa paruse un melange de serpules, de coguilles, ce poìpicrs; le premies rour eft dégarni. - Trochus conchyiophorus. Ginel. Eosc. Lorn.

2. 3. * Xe nophore à trois côtes, turriculée, garnie de coquilles, trois côtes ciftinctes sortant de l'ombilic.

Xenophora tricostata nihi. - Tocins conchyliophons aliorum.

L'un des ibdividus porte des sabots, Jes caverares rour écs ve:s le sommet, et dis opercules; l'autre des bivaves, il es: grand et bien conservé.

4. 5. * Xenopjore vol c a n i que, turriculée, les tours de spire colies par des criblux de laves, la base présentant cinq côtes intermétiaires scrtant de l'ombilic.

Xenophora $v$ ulcan du cailioux de laves de tout gente. L'un des Incivious of grand ct rés peo

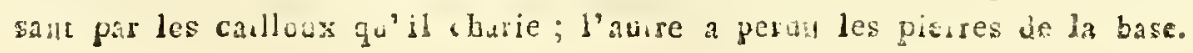


$114 \quad C A D R A N . S O L A R I U M$.

6. * Xenophore mćandrine, fubturriculée, les tours de spire garnis de méandrines, la base à côtes nombreuses ne laissant point d'espace entre elles.

X enophora mec a ndrina mihi. Je rrouve les côtes de la base variables, et cependant concordantes dans des especes femblables, voici pourquoi j'en ai ciré le carastere fpécifique, mais je ne dois cependant pas dissimuler, que les côtes varient d'après la quanti é d'objêts étrangers qui garnişent le prẹmìr tour de la coquille. Elles paroissent être les conduits des vaisseaux ou au moins les endroits où font artachés les vaisseaux conducteurs da iiquide calcaire le. qnuel colle ces différens objêts.

\section{Cadran. Solarizus. L a m a r ck.}

Eoq. en cóne deprimé, ayan dans fa base un ombilic ouver, crénelé fur le bord interas des tours de fire. Ouverture piesque quadrangulaire.

Animal, inconnu.

1. 2. Cadran perspectif, convexe, les tours de spire ob. tusement marginés, ftriés longitudinalement, 1'ombilic crénelé.

Solarium perspecivum, Lamack po 85 - Trochus perspectivus Lin. Bosc. - Chemnitz 5. t. I 72 . f. 1691. 16 ; 60 .

3. Cadran hy bride, convexe; les tours de spire hisses, l'ombilic sub--crénélé, plissé.

Solarium hybridum. - Trochus hybridus Lin. M. 645. n. 3̧o. Bosc. 4. p. 149. 150. Chemnitz 5. t. 173 . fo $1702^{\circ}-1703$. - Se trouve daus la n.er Mediterranée.

4-8. Cadran solaire, convexe, les tours de spire radiés par des larges épines, l’ombilic finement plissé.

Solarium radiatum, mihi. - Trochus folaris Lin. Mus. Lud. Uly. 645. R. 328. Bose. 4. 552 - Chemnitz 5. 8. 173. f. 1700 . 170r. t. 174. f. 1716. 1717. - Se trouve dans la mer des Indes. L'un des plus grands content une trés peute écrevisse bronard. 
Sabot. Tarbo Linnaei.

Coq. conoide on turriculke: l'ourerture arrondie, entière, et fans deats à la columelle.

$L \curvearrowright s$ denx bords désunis daas leur gartie fupérieure.

Saboier V. Lam. p. 86.

* Neritoides, dont le bord de la columelle est plat et imperforé.

1. Sabot vignot, presque ovale, pointu, ftrié.

Turbo littoreus, Lin. fn. fuec. 216\%. Busc. 4. 124. P1. 32. f. x. 2. Cheme nitz. 5. t. 385. f. 1852. 1853. 1855. - Se trouve dans l'ocean européeir.

2 - 5. Sabot boson; blanc; et des variétés branes et demibrunes.

Turbo muricatu Gmel. Eosco Borno to 12. f. 15. I6. Chemnitz 5. t. 17?

f. 1752. 1752. - Se trouve fur les côtcs d'Eurrope d'Afrique et ả' Anérique.

- Solides imperforéso

6 - 10.9 s b ot pét holate, ovale, uni, brillant; les tours de spire presque anguleux vers le laat.

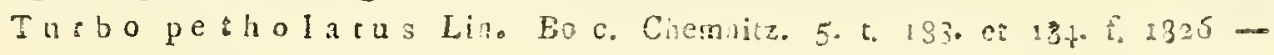
5839. - Se rrouve dans les mers de i'Inde et de l'Amórique.

I1. I2. Sabot chrysostome.

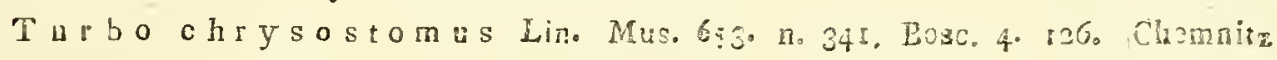
5. t. 178. fo $1766 .-$ de la mer des Indes.

13. 14. Sabot pagode.

Turbo pagodus Lin。 ib. Bose Chemnitz. 5. 6. 163 . 5. I54. $154^{2}$ - - de la mer des Indes.

15-1\%. Sabot éperon.

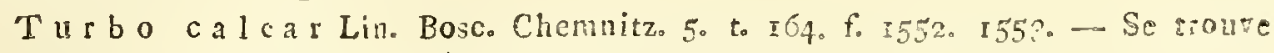
dans la mer des Indes. Les deux petits individus paroissent difírer non comme varictés mais comme cspèces.

$18-21$. Sabot marbré.

Turbo marmoratus Lin. - Chemnitz. 5. t. 179. F. 7775. I776. De fuperbes individus.

22 - 26. Quatre moitiés et un fragment nacrés de la même espéce.

Les chinois en font des vases à boire, et les ornent avec des gravures. L' un porte une inscription en caractéres chinois. 


\section{$\mathbb{1}: 6$

27.23. Sabot farmatique.

Tubo fasmaticus Gne! - Chemnitz. 5. t. I79: fo In7?. I778. I78?. (L'un est tout La faik et l'autre à demi depouilic.) = Ee trouve dans les mets d'Ajrique et des Iindes.

29. Sabot cornu。

Turbo cornutus. Gmel. - Chemnitz, 5. to I79. f. 1779. I7880 - de la mer de la Chirie.

30-33. Sabot radié, roguens, les tours de fpire cylindriques distans, ftriés transversalenent, avec de petites épines imbriquées.

Turbo radiatus Gmel. Posc. Chemnitz. 5. to r8c. f. 1788, 1789. Deus en font couvert d'opercules et de feupules. - Se trouve dins la naer rouge.

34. Sabot canaliculé。

Turbo cansiliculatas Gmel. Bosco Chemaitz. 5. to I81. f. I794. - Sa tronve daus la mer des intos.

36. 3\%. Sabot épervier.

Turbo fparverius Gmelo Bose. - Chemmilz. 5. t. I8r. b. 1798, - Se trouve dans la mer des Indes.

38. - 40. Sabot fpénglérien, (blanc, varié de jaune); les tours de fpire cylindriques, firiés transversalement, avee un canal intermédiaire.

Turbo fpenglerianus. Gme1. Bosc. Chemn. 5. to I8t. f. i80r. r8c2. Se trouve daus la mer des Indes.

4r. Sabot émeraude, variété à ftries d'éméraude.

Tu rbo fmaragdulus, testa ponderoar fublepressa (laeviussuia?) obllique rngcsa: fpirae anfiattibus quatuor; primo terete ampliore. Gmel. $3595 \mathrm{n}$. 30. Bosc. 4. 131. - Chemnitz. 5. t. 82. f I. 1815. 1816 . de la nouvelle Zilanle.

a. Sabot helicin.

Tuzbo holicinus Born. Test. p. 355. 355. \&o I2, f. 2 s. 24. * Solides perforés.

$43-47.5 a b$ ot pie, conique, arrondi, uni, arec uno petite dent à l'ombilic. 
Tusbo pica Hin. Bosc. - Chemnitz. 5. t. 175. f. I750. I75I. Les figures I 420 et I 421 t. 15 citées par $G$ melin et $B$ osc ne présenteat pas la conuille, mais for opercule. - Il fe suave dans toutes les mers.

48. S a bot sanguin.

Turio sanguincus Cmel. Bose. Chemnitzo. 5. 8. $2 \% \%$ f. 1756. I75\%. des côtes d'Afrique.

49. 50. Denx sabots depouillés et nacrés.

Les espèces fossiles voy. vol. II. p. 270

Monodonte. Monlodontr. I, a mate:

\section{proches tin na e i.}

Soq. ovale ou conoide. I'ouverture arrondie, entière; mais murie d’une dent, formée par la base sailiante et tronquée ou raccourcie de la columeîle. Les deux bozds đésunis supérieurement.

Aninat. v. Lamarcle p. $8 \%$.

I. Monodonte retan, ovale, les tours de spire peu sensibles, à côtes transversales applaties, ftriçes longitudina. lement.

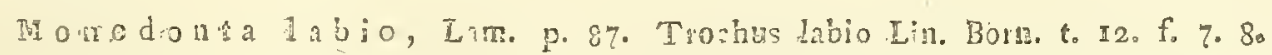
Cbemiatz. 5. t. 166 . fo 1579.15 50. Bose. 4. 167 . Pl. 31. f. 6. la double bouche de. Roissy. 5 p. $285 .-$ Se trouve dans la mer des Indes.

2. Monodonte canaliculée, ovale, les tonrs de spire separés, canaliculés à côtes transversales, aiguës.

Monodonte canalioulata mihio

3. Monodonte bicostée, avale, les tours dé, spire diso tincis, à côtes grainucs diftantes, doux cle chaque tour plas saillantes.

Nonodonte biconta mini.

4- Monodonte granuleuse, ovale, à tcurs de spice peu difincts, à côtes granuleuses contigues, onbiliqués, la dent fe prolongeant avec la columelle. 
Monodonte granulata mihi. Une tiès belle espèce de Monodonte rougre, a côtes granuleuses très serretes, dont deux de chaque tour sont ponctuées de noir. Le quatriènc individu présente une variété srès jolie, en ce que les cordons en chapelet sonr noirs et blanes et en ce que les cordons font plus fréquens que dass les autres. - Elle paroir la méne avec Trochus pharaonis Lin. le bouton de camisole de que gies auteurs, le vaser d'Adanson。 erc. Mais si cette singulière forme de la columelle eft confante, elle' n'eft ni Toupie ni Monodunte, mis elle doit former un gente particulier.

\section{Da ph in ule. Delpbinzla. Is a m.}

\section{- Turbo L i n na i.}

Testa univalvis, fubdiscoidea vel abbreviato-conica , solida, margaritacea, umbilicata : anfractibus fub-asperis. Apertura rotundata: marginibus orbiculatim connexis. Lanarck. Anal. du Mus: 4. p. 108. gen. 30.

Animal inconnu.

I - 4. Da u phinule ép in e us e, l'ombilíc épineux, les tours de la spire avec des pointes rameuses.

Delphinula spinos a Lam. de Roissy 50. p. 290. t. 54. fig. 45. Turbo delphinus Lin. Bosc. - Lifer : 608. f. 45. Gıalt. 68. f. c. D. Chemnitz. 5. 4. I74. f. 1725 . 1726́. 1736. Cyoloftoma del phius Lam. An. fo vertebres. p. 87. Se trouve dans la mer des. Indes.

\section{Cyclostome. Cyclostoma $\mathrm{Lam}$ a rck.}

\section{Turbo et Helix Lin.}

2este univalvis, discoidea: vel convexo-conica, fubdiaphan: anfractibus cylindraccis. Apertura circinata : marginibus orbiculatim connexis: acutis, patenti-reflexis.

Lamarck. An. ib. p. 112, gen. 3I.

Animal voy. de Roissy. p. 295.

I. 2. Cyclostome élégant.

Cycloftoma elegans; Lamarck. de Roissy. 5; p. 295. t. 54. f. 5: Turbo clegans Lin. Bosc. Gualt. s. 4. f. A. B. 
3. 5. Cyclostome rubanné, brunâtre; ombiliqué à tours globuleux, fasciés de rouge-pourpre.

Cyclostoma faseiatum mihi; - Turbo ligatus Chemoitz. 5. to 123. f. $107 \mathrm{I}-1075$.

6. Cyclostome bordé, brun, à tours globuleux, la bouche entourée de deux bords renflés.

Cy clostoma bic inctu m mihi. Le lieu natal en est inconnu.

7. Cy clostom a marginé, blanc, ombiliqué, lisse, à tours globuleux, 'la bouche marginée d'un contour mince et tranchant.

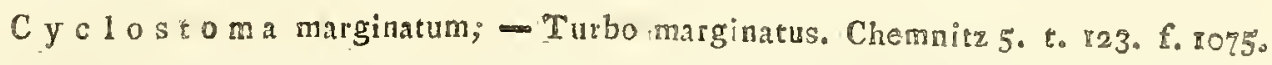

8. Cyclostome lisse, blanc, ombiliqué, sillonné, à tours globuleux, la bouche lisse.

cyclostoma la evigatum mihi. J'ignore fon pays natal.

\section{S c a l a r e. Scraria L a m a r c k}

\section{Turbo Linnaei.}

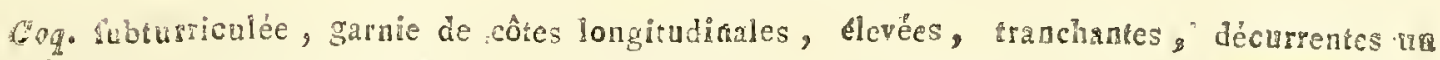
peu obliquement dans touce la longueur de la fpire. Ouverture arrondie: les deux bords rẻunis circulairement et têtéchis.

Animal. Voy. Lamarck. p. 88 .

I. Scalaire conique.

Scalaria conic a Lam. p. 88. de Roissy. 5. P. 300. pl. 54. f. 6. Turbe fcalaris Lit. Cyclostoma Salaris Bosc. 4. 85. p!. 32. F. 3. - Chemnitz. 4. to

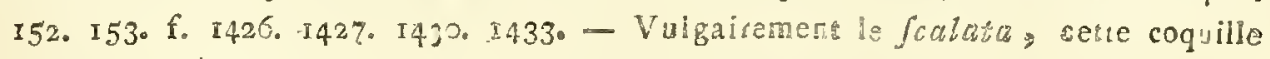
est aussi précieuse que fon prix augmente avec fa grandeur. La nôsre a 88 lignes. - Elle fe zrouve dans !a mer Médicierranés.

2-6. Scalarie grille.

Scalaria clathes Lam. de Roissy. Turbo clathrus Linn. Cyclostoma clathra. Bosc. - Chemnitzo 4. to 153. fo 1434. I438. dans Ia Médio zerrane et dans 12 mer-des Indes.

$$
2^{*}
$$


Maillot. Pupa $\mathbb{L}$ armarck.

Turbo Linnei.

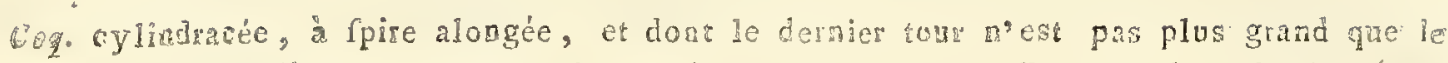
penultième. Ouvertur incégulière, arondie on ovale, les deux bords rérnis circulairement.

Anstral incounu.

I. ¿ Le marllot, alongé, blanc, à donze tours, sillonrés lon gitudinalement, les sillons écartés.

Pu pa uy a Lamazck p. 68 . de Roissy. 5. p. 358. t. 54. f. xt. - Turbo uva? Lin. - Cual. to 58. f. D. Bulimss popa. Bosc. 4. II3.

3. 4. I a momi e, alongée, brunâtre, à dix tours sillonnés longitudinalement, les sillons rapprochés.

Pupa mumia, de Roissy ib, Chemnitz. 4. t. 153 . f. 1439. Bulirsus mumia. Rose. ib.

Turritelle. Turritella $\mathrm{L}$ a marck.

Cog. tursiculée. L'ouverture arrondie et ayant les deux bosus désunis supéricurement. Bord drois muni d'un sinus.

Animal v. Dargenville. Bose. 4. p. to.

I - 6. Turitel le tar i è re, la spire chargée de cinq à neuf carènes aigues.

Turitella terebra Lamarck. p. 8S. Bogc. 4. p. 80. P1. 32. f. 4. 5. de

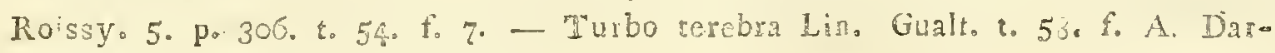

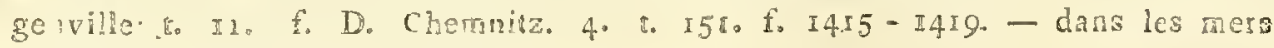
d'Europs, d'Asie et d'Afrigue.

7. 8. Turritelle duplicate, les tons de spire ayant dans leur milieu deux carènes aigues et rapprochées.

Turrirel Ia duplica a, Rosc. de Roissy. - Turbo duplicatus Lin. -

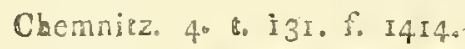

9:- Turritelle inzriquée.

Tarritella imbricata, Bosc. - Turbo imbricatlis Lin. Chemaiz 4 .

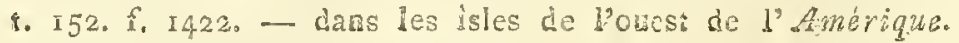

To. Turritelle repliée.

Tursitella replicata Bosc. de Roissy. - Turbo replicarus Lin. Chemnitz. 4. 8. 155 . fo $x_{45} 20$ - dans la mer des' Indes. 
II- 13. Turitelle exolète.

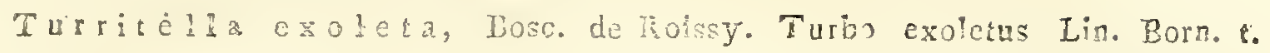
I3. f 7. Chemnitz. 4. t. I52. fo I420. 1425. - sur les còes de Gutinée. Gmelin et Boss font de l'exoletus de Bor n wne espeoce sous le nom $\mathbb{T}$. de obsolctus, wais ce n’eft giune simple tariéré.

R4. I 5. Turritelle polie, imperforée, tyès unie, blanche: louverure ovale.

Turatella polita Bozc. - Tubo policus Gmel.

16. I\% Turitelle calcaire, blanche, épaisse, les tours legèrement sillonnés, le sommet obtus, l'ouverture oblongue.

Turitelia calcarea Born Ind. p. 402. Test. p. 389. t. 16. f. Is. Chemnitz. 9.

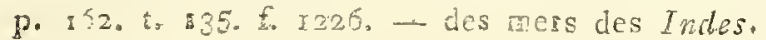

18-20? Turritelle tronquée, glabre, les tours de spire arrondis, striés, I'ouverture ovale.

Turritcila dacolląa - Helix decollata Iin. - Chemaitz q. p. I.82. t. I36. f. 1254,1255 。

Ianthine. Inmbina Lamared.

He lix lin.

Cog. fubiglobulerse, diaphane. E'owverture trianguhire. Un sinus angolews an bord drois.

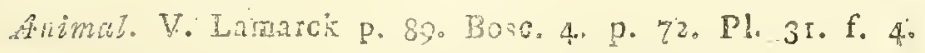

I-10. Ianthine fragile.

Ianthina fragilis, Lamarck. Bosc. de Roissy. 5. P. 394. 1. 55. f. IT. Felis janthina Lin..- Chemritzo 5. to I66. fo 1577 . 1578.

B a 1 e. Bulia L i n n a e $\dot{x}_{0}$

Coq. bombée, à fpire non fallarte et à burd droît tranchant. Ouverture aussỉ longue que la coquille. Foint d'onbilic inférieurements.

Animal. V. le genve bullée p. 9 .

3 - 6 . Bulle a mpoule, ovale, bombée, opaque, hise; le sommet ombiliqué. 
B ull a a m pull a Lin. Lam. Bosc. 4. 66. P1. 30. f. 5. de Roissy 5. p. 325. ז. 55. E. I. Martini。 1. E。 21. fo 188, I89. 190. - Vulg. la muscade; - dans Ia mer des Indes.

7-9. Bulle striée.

Bull a firiala Lino Bose; de Roisey; Marsini. ro to 22. fo 202. 1204. fur la côte d'Afrique, et des Antilles.

10. Bulle papiracée.

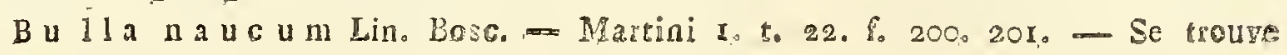
dans la mer des Indes.

II? Bulle épaisse.

Bulla folida, Lin. Bosc. Chemaitz I0. to I46. fo 235601357. - dans la mer des grandes Indes.

12. Bulle o u verte, presque orbiculaire, légèrement firiée, blanche; l'ouverture très baillante.

B ul d a a p e rs a Lin. Bosc。Chemn. Io. to 346 . fo I354. I355.

I3 - 15. B u 11 e de C e y I a , presque cylindrique, ftriée, cor née, diaphane; les sutures canaliculées, louverture trè baillante presque triangulaire.

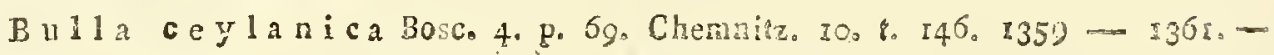
dans la mer des Indes.

Bulime. Bulizus Bu gui ère.

Coq. ovale ou oblongre, ayant le dernicr tour de la fpire plus grand que le penulsieme. Ouverture entière, plus longue que large. Columelle lisse, fans troncature es fans évasement de fa base.

Animal, Voy. Lam, p. go. Bosc. 4. p. 89.

I - 3. Bul ime à boụche rose, oblong, garni de ftries longitudinales; la Ièvre droite, la columelle couleur de rose.

Bulimus haemastomus Lam. p. go Bnlinus oblongus Bose. 4. p. roo. - Lister t. 27. f. 2r. Chemnitz 9. E. İg. fo 1022. 1023. - Se trouve dans les forêts de Caynne. Vulgairement: la fausse oreilie de Midas.

4. 5. Bulime ovale. 
Bulimus ovatus, Bose. - Chemnitz. 9. t. Iig fo 1020. 102I. Se troure rerresece aux Grandes Indes.

6-9. Bulime poule sultane.

Bulimust a datus Buguière. B sc. - Chemitz. go to IIS. fo ror 5 . ror6. - Se ravure rerrestre au mếme endroit.

10. I I. B u I ime g a che, ombiliqué, oblong, diaphàe, blanc, fascié; l'ouperture tournée à gauche et alongée vers le bas. Bulim its 1 a evus, Bosc. h. I00. Lister t. 33. f. 31. Favarne p. 65. f. A. 3. Chemnitzo 9. Io IIIf fo 940.949\%. Ties raie; des Grandas Indes.

I2. 13. B $u$ I ime citrom, ovale, lisse, couleur de citron; la spire tournée à gauché. la lève gauche burdée à l'extérieur par un bourrelet blanc.

Bulimus e itrinus, Bosc. Helix perversa Lin - Chemritz. g. t. r 10 . f. 928. 929. - Se troute dans l'Amérique méridionale. L'une eft tounéc à gauche, l'autre à droire.

ॠ4. Bu lime inversé, oblong, là lèvre gauche recourbée en dehors; la spire tournée à gauche.

Bult mus inversus, Bose. Gialt. t. 5; f. O: Favanne. t. 8J. fo N. Chemnitz. 9. to I10. fo $925 \cdot 926$. - Le nôtre est tout à faic blanc fans les flammes longitudinales clont on ne voit que de foibles iraces.

Agathine. Achotina I a m a r c k.

\section{Bu I a Lin na e $\dot{i}$.}

Eag: orale ou oblongue. L'auvernure entière glus longue que large. Columelle lisse, tronquée à fa base:

Animal. V. Bulimus Zebra Brug. Dicto n. Ico.

I. 2. A gath in $\mathrm{z}$ èbre, ovale ventrue, Blanche, marquée de flammes noirâtres; la columelle arquée, blanche.

Ach at hi in a Zebra. Lam. Bulla ze'ra Gmel. 343I 3r. Butimus Z óra Bruga, Do:c. 4. II6. - Dargenville. Supl 2. f. L. Fa:amie pl. 65. f. M. 3. Chemnitz. 9. t. II8. f. Iorf. - Se rouve cerrestie' $M$ Madagascar.

3-6. A gathino pourprée. 


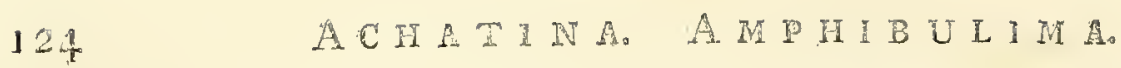

Achatina purpurascens; - Bulla pupurea Gmel Bulim:s purpum rascens Bose. 4. x17. Lister. t. 581. fo 35. Chemnitz 9. to 218. fo 1017. 1018. en Afrique ct dars les Antilles.

7. 8. Agathine variée.

Achatina vasiegata Lam. p. gr. Bulla achasina Lin. Bulimus achatis

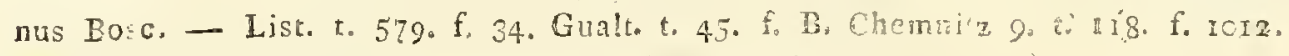
wor3. Vulg. la perdrix. - dans 1'Amérique méridizonate.

$9 \approx$ 12. Agathine pavillon。

Achatina yexillam; - Bullae virgir. varietas Lin. Bulimus verilum

Bosc. - Favanne pl. 65. f. G.2.3.6. Dargenvo 14. f. M. Chemnirz 9. to $17 \tau_{0}$

f. 1004. 1006. - des Grandes Indes.

I3 - 15. V ariétés dè la mème espèce.

16. Agathine vertrue.

A chat ina ventric os a; - Bulimus rentrieosus Bosc. 4. I29. Chemnitz.

9. t. II . f. 1007 . Icc 8 .

$17-24$. Agathine ruban, conique lisse, hanc fascié; la columelle arquée, couleu de rose; l'intériew de la levre gauche plissé,

A chatina virginea; - Bulla virginea. Lin. Bulimus virgineus Bosa. 119. Chemnitz. 9. t. II7. E. 1005. 1003. t. 173. f. 1682. 1683. - de Cayenme. Bosc. 4.99. fraic mention d'une antre espèce fous le même nom die Bulimus vigra gineus, Fuline incarnat (Chemairz 9.t. I21. fo I042.) mais celle-là appare t.ent auz vais bu li mes.

25. La même contenant une écrevisse bernard, à pinces très courtes et grosses.

20. I'agathine ruban tenant une autre, femblible, plus petite auprès de l'ouverture.

Amphibulime. Ambrbulina I. am.

Coq. univatue, cvale ou ovale conique. Cuverture entiere, plus longue que large, bord droit tranchant, non réflechio, remontrint fur la columelle fans félargin et fe sonfondant avec elle. Colmmelie fius pli, point d'opercule.

Animat inconus. Voty. Lam. Anal, du Mus. T. 6. p. 304. P1. 55. F。 I. a.

1. Amphibulime en capucllon. 
Amphibulima cucnliata, ovato-inflata; ftriis oblique transversis: spira obtusa, brevissima, rubra.

Lam. ib. p. 3o5. Succinea Drapannata Tabl. des moll. po 55

Ĺ y m née, Lymmaea. Ia marck.

Coq. cblongue, fosturriculée. L'ouvertuse entière, flus longue g"e large. Partie infério

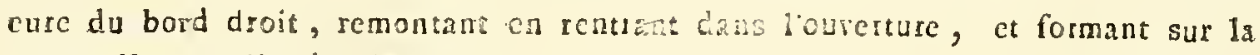
columclle un pli très oblique.

Alizinal. voy. Lam. p. gr.

I - 3. I y $\mathrm{m} n$ é e $\mathrm{stag} \mathrm{n}$ a l e, cblongue, ventrue, rransparente; la spire longue, étroite, effilée; le bord de la lèvre dronte onduleus.

Lym naca stagnalis Larn. p. gr. Irelix frogralis Lin. Iulinus ftagnalis Tos 3. 4. 95. El. 32. f. 1. 2. Lifter t. 2. fo 2I. Gualt. 1. 5. f. 1. Favarne plo 61. f. I5. Chemuicz, 9. \&. I35. f. 1237. 1238. - en Europe.

4. 5. I. ym ée des marais.

Lymnaca pelustris; - Eulimus paluftris Eosc. Chemitz. 9. 6. 155. f. I230. 12द्go. - en Europe.

Melanie. Melaña Lamarck.

Coq. turricrilée. Louverture crtilire, plus longue que large, crasée a la base de a colamclle. Aucur pli sur la columelle.

Animal inconnu.

1. Melanie tiare.

Mílania a marula Lam. p. qr. Helix amaruia, Lin. Brrgu. Bose. Bonis

Mus. t. 16. f. 21. Chemaitzo 9. to 1E4. f. I2:8. 1219.

\section{Pyramidelle. Paranidtila La m.}

Co7. turriculée. I'onveriure cariè:e, ecri-orale. Columclle saillante, munie de trois plis transverses et perfuréc ì sal base.

\section{Animal inconnú.}

I. Pyramidelle polie.

Py ramidella dolabrata. Iam. p. 92. Trochus dolabratus Lin Bulimue do'abratus Brug. Posc. - Cheminiz. 5. \&. 167. f. 1603.1604. - cn Afrique. 
2. Pyramidelle foret.

Py ramidella terebellum; - Bulimus tarobellam Biug. Bosc. Listo

to E44. to 72. Gualt. to 4. f. M. - dans les isles de l'Amérique.

\section{Solidule. Solidula. Mihi.}

Coq. oblougue. L'ouperture cntière, très longre, pyramidale. La lèvre droite solide, dépassaur la columclle, qui eft perforée et manie de deux plis et d'uu sinus tuès profond.

Animal inconnu.

I. Solidule mosaíque, ovale, étroite, les spires fub-canaliculées, ftriées transversalement, blanches, monchetées de noir.

Solidula mosa ica; - Bulimus solidulus Brugu. Bosc. La forme de la Columielic nous ollige d’étahlir un genre particulier. Lill né lui-mêne etoit douteux fur fa vraie pace; il I'a place farmi les bulles, (ed. 10. p. 728 n. 346 ; - Mus. reg. Lud. U.1r. p. 590. 12. 228 . bulla folidula) et plus tard i1 en a fait une Volute. (Voluta soliclula cd. I2. p. 1187. n. 395.) Mais elle. ne peut être non plis un Butime, parcequ'elle n'en présente aucune sessemblance. Une ronne figure Voy. Chemnitz. 10. p. i5. t. J49. f. r405. Notre. cxemplaire on un peu plus petit es pilusalongè. - Elle fe trouve aux Grandes: Indes. - Au rểme genre appartionnent les espèces fuivantes: Voluta toro matilis, et coffea Linn. (Eulim. Erugo et Bosc) - Bulimus monile, ovuinss. 2ariegatus, Dosc. 4. rog.

\section{A ur icule. Auricula. La marck.}

Cogo ovale ou oblongue, a fpirc faillante. L'ouverture èntière plus longue que large, résécie fupérieurement. Un ou phisieurs plis: fur la columelle, indépeudian de 12. décuraence du bord droit fur la base du burd gaulhe.

Animal inconnu.

to. A uricule. Midas.

Aur icula Mida e Lam. p. 92. de Roissy. 5. p. 362. Pl. 55. f. 7. Volua auris Midac Lin. Bulimus auris Mi tae Bruguière. Basc. Dargellville pl. 10. E. G. Favanne pl. 65. f. H. 20. Manini 2. to 43. f. 436 . 438. Cet exeinplaire est d'une grandeur exiraordinaire, il a plus de quatic pouces de longueur et presque deux de largeur. - des Indes orientales. 
A U R I C U L A. POE IODON

2. Auricile lisse.

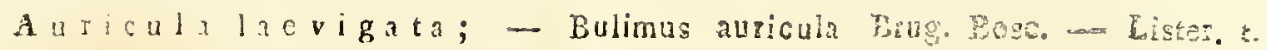
57\%. 32. Gualt. t. 55 Fig. F.

3. Auricule tortue.

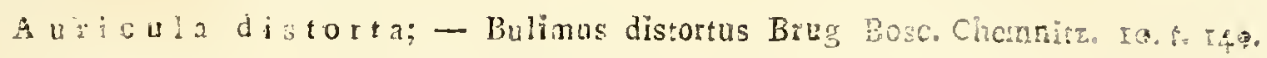

f. 1395. \$395. - des Grandes Indes.

4. A uricule papyracée, ovale, mỉnce, transarente, les sons de spire arrondis, louverture étroice, lit columelle garnie à un seul pli.

A uricula papy race a mihi. Uae espèce trés rate qui on refté incono nue aux Naturaliftes. Elle est bianche, d'un blanc sale rirant sur le vert, rranspar nte, et sa ftrocture parcî̀, regurdée au jour, commè une gelatine dur. cit. Elle a i p. 0 lo de longueur, sur 8 lignes de largeur. Le liey nata! es cest inconau.

\section{Polyodonte. Polyodonte $\mathrm{M}$ ih i.}

Cuq. ovale, compriméc, ombiliquée, à spire saillante; les tours de spire sub-anguleux, comme interrompus et soudés latéralement, luuverture oblongue, presque de= mi - circulaire, dentée des deux cotés.

Animal. Voy. Adauson 2. por.

I. Polyodonte aveline, ovale, épaisse, l'ouverture garnie de sept dents.

Polyodonte scarabaeus; mihi. Une coquille terreltre, des Giandes Indes, très rare, qui a beaucoup occupé les Nacuraliftes; - Heliz sca abúeus Linna e i. Mus.663. n. 36 . Gincl. $36 \div 3 . x$.

Lister. t. 577. fo 3r. cochlea compressa varicgata, lataribus acutis, senis, minimum, dentibus donata.

Gual t. test. t. 4. fo 5. turbo terreftris insigniter ventricofus, vabilicatus, e: variegatus. Fig. bonne.

R u $\mathrm{mph}$. T. 2\%. l. I. cochlea imbrium ex montbus Ambinac.

$\mathbf{P}$ et i ver Gazophylacium 1. b. 5. f. ro. Cocklea bengalensis ore lacero.

Favart d'Herbigny Dist. t. x. p. 57. t. 2. p. 20. ave'ine ou guelle de loup; apertura utrinque singulariter deratata.

B or $n$ Ind. Mus. p. $34^{1}$. Test. p. 365 . Vigin. p. $3^{\Re} 4$. I a.

A ig caville t. 9. f. T. Buciin garni de dents de deux contés.

$2 *$ 
Favanne Conch.t. 65. F. D. I, D. 2. D. \&.

K norr. Del. 6. t. I9. f. 2. 3. bonne figure.

Schrotes Eint. 2. p I22.

Chemnitz. D. p. 179. t. I36. f. 1249-1250. Z1uber-Regen. U. Sturm Schnecke; figure très mediocre; Bosc. Coq 4. I 1 c. B ulime aveline, ovale mazqué de deux angles longitudinaux, l'ouverture garnie de sept dents.

2. Polyodonte piétin, ovale épaisse, ftriée; l'ouverture garnie de cing dents.

Polyodonte pedipes; - Bulimus pedipes B.s: 4 Iro. Pedipes Adansın. pl. I. f. 4. L.fter. t. 577. f. 32. Chemnitz. 9. \&. 1336 to $1251 .-53 a$ la coupe horizuntale eftaintéressaute, parceque on y voit la culumelle creuse.

\section{Volvaire Volvaria La marck.}

Coq. cylindrique, rauiée sur elle-même, sass spire sallane, ouverture étroite, aussỉ longise que la coquille. Un ou plusicurs plis sus la base de la coluinelle.

I. Volvaile bullo ide.

Volvaria bu110 ídes Lam. p. 93. de Roissy. 5. p. 328. P1. 55. f. 2.

\section{Am pullaire. Ampullaria 1. a m a r ck.}

Goq. giobuleuse, rencrue, ombiliq̨uée a ra base, fans callosité au bord gache. Ouvertuse entière plus longue que large.

Animal: Gastéropate fluviatile, muni d'un opercule corté.

I. Amptilaire ido Ie.

Ampullaria rugorsa Lam. p. 93. Pulimus urceus Eosc. Brygll. Ampulo la.ia urcea, de Roissy. 5. p. 37T. Pl. 55. f. 8. - dans les fieuves de I' $A$ mé"rique.

I. 3. Ampul la ire cordan ble u.

Ampullaria fasciat a. de Roissy.5. ib. - Bulimus ampullaceus Brugu. Bosc, - Ife:ix ampulliceus I.jnı. Chemniz. 9. t. 128. f. II53. I135. - daus les caux douces de l'Inde et des Antilles.

Pla norbe. Planorbis I. a marck.

Coq. discoide, a fpire non rallante, applatie ou cnfoncée. L'ouversurc entière, plus longue que large, échancrée latéralement par la fallic coavexe de l'arato dernier rour.

Animis V. Lam. p. 23 
3. 2. Planorbe corne de belier, applatie; íourerture ovale.

Planorbis cornu árictis Lam. ib. Helix cornu arictis Litd. Bosc. Chemnirz. 9. r. 112, f. 952. 953. - daus les mers de la Chine.

3. 4. Pla norbe cor-de chasse, applatie, brunâtre, I'ouverture ronde.

Planorbis or aea de Roissy. 5. p. 375. 2. 55. f. S. Helix cornea Lin. Bose. 4. 24. t. 29. f. 3. - Chemnitz. 9. t. $12 \%$ f. Irzo.

5. 6. 7. Planorbe contournée, applatie, des deux cotćs; l'ouverture linéaire, courbée.

Planorbis contorta; - Helix contorta Lino Bosc. Chemnirz. 9. t. Iz?. f. $1 \& 26 .-$ en Euroue.

\section{Pleurodonte, Plezrobionte. Mils}

Eaq. discoide, à fpire obtuse, peu élevéc. L'ouverture ronde ou oblongue, garnic d"une ou de plusicurs dents forméas par des plis de la lève extérieure.

Animal, incorau.

I. Pleurodonte sinuée, brune, lpeu carénée; $1^{\text {souverture }}$ oblongue, à Iẻvre renflée. Quatre dents et trois plis exté. rieurs à la lievre droite.

Pleurodonte finuatam; - Helix finuata; Gmelin. Bosc. Vulgaircraent fa noisente dentée. La lampe antique aumée de quatre deniso

Lister to $9 \%$ fo 98 . et to 98 . fo 990

Klein Meth, ostr. f. 3r. lit. d. po I1. Angystoms tetracdon.

shane fam. \&. 2. r. 22\% n. C.

Eavanc. 8. 63. fo 8 .

Se trouve en Amirique.

2. 3. Pleurodonte I y chnuque, imperforé, blanche, avec des fascies brunes; l'ouverture oblongue, transversale, bidentée. Un seul pli à l'extérieur très alongé:

Pleurodonte lychsuch us; nihi. - Helix lychonchus Cinelin. Bosc. - Lister. to yo. fo g20 on ignore fon pays nan!. 
4. Pefrodone I a crne, imperforé, blanche, applatie an dossus, renflée ca dessous; l'ouverture oblongue, biden tée; ta plí trés court à l'extérieur.

Plegrodonte lucesua mini; - Nolix Jucerna et lampas Gmelis. Bosc. - Cheniniz 9. 20 I25. fo 1108 . Irog.

5. 6. Pleurodonte inégale, perforée, convexe en dessus et en dessous, les tours friés, inégaux, du coté de la bouche (bidentée), plus hauts et plus gros que de laatre. Un pli très profond à l'extérieur formant un trou rriangulaire.

ple urodonte ina equalis mihi. Coquille très rare et la plus grande de fon genre (I pouce 2 lignes de hau fur 2 p. x. 1. de large) que poin de naturalisse n'a décrite. J'ignore fon pays wa.al.

7. Pleurodonte la mpe, imperfore, frune, convese de deux cotés; la houche transverszie, fub-bidentée, le pli extérieur insensible, se perdant dans le renflement du bord.

Pleu rodonte la picida, mihi, Holix lapicida Lin. Bosc. - Chemnitz. 9. t. 126. f. 1107. - Se trouve dans les bois d'Europe.

8. Pleurodonte isognomostome, convexe de deux cotés, ombiliquée, imperforée, l'ouverture oblongue, tridentée. Point de pli extérieur.

Ple urodonte isognomostomos; mihi. - Helix isognomostomos Gmer. Bose. - Lister to 93 f. 94 . Se trouve en Vivginie er en Allenagale.

1

Anostome. Anostoma. Mihi.

Coge discoide, à fpire applarie; la bouche tournée en haut ou du coré de la fpire, et garnie de dears des deux cotés.

Animal inconnu.

x. Anostome à huit dents, l'ouverture présentant un péristome circulaire et renflé, cinq grandes et trois petites dents. 
Anostoma octodentata mihi.

Helix ringens Lin. X11. 1243. n. 604.

Lister. t. 92 . f. 1 co.

Argenville. t. 28 t. 13.14.

Born. Indes p. 378. Test. 369 . t. I4. f. II. Iz.

Favanne 1. 63. f. 5.10 .

Chemniz. 9. p. 86. t. 109 . f. 9I9. 020.

Busc. 4. I . Helice ridée; Helix ringens.

C'est une coquille des plus extraordinaires qui fe crouve terrestre aus Indes. Notre excmplaire est d"nne grandeur rare; il a 9 lignes de hautcur et uis pouce fix lignes de largeur.

3. Anostome à fix dents, l'ouverture ne présentant que le bord renflé de la lère droite, et 6 dents de grandelir presque égale.

A nostoma laexodon mihi; -

Klein Meth. ostrac. §. 3r. lit. e. p. I I. no I. Aingystoma hexodon.

Davila Catal rais. I. p. 4.40. n. 985.

\section{H e I i c e. It tion I, i $\mathrm{n}$ n a e i.}

coq globuleuse ou orbiculaire, à fire convexe ou cunoide. Onverture entère, plus large que longue, échancrée fupéeicusement par la failhe convexe de l'avant dicrnicr tour.

Animal. Vou. Lamarck. 1. 94.

- Helices à fpire applatio:

I. He lice peau de serpent, peu carénée, peinte de fascies et de flammes reuges et biancles, ceintes en dessus. de quatre rangs de pointes; l'onverture frangée.

Helix pellis ferpentis, Gmelir. Bt:se. - Chemnitz. 9. p. 79. to 125. f. 1095. reg6. - Se row e seriestre en Amérizue.

2. 3. H. a e goplut a $1 \mathrm{~m}$ e, crbiliquée, un peu comprimée, verdâtre, sans tachés, avec sept to urs de sprie.

Helix aegoghthalmos Gmol. Bo:cu - Argenvile 6. f. Ev - Se troure aux Indes。 


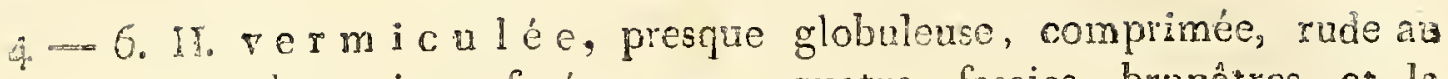
roncher, imperforée, avec quatre fascies brunâtres et la Jère blanche, recourbée.

Hel is vermiculana. Gmelin. Bosc. Favante t. 6.4. F. K.2. K. 2. Se irouve eerresire en Italie et en Espagne.

7. 8. I. I asciée, deprimée, ombiliquée; à zones brunâtres, I’ouverture transversale, la lève peu recourbée.

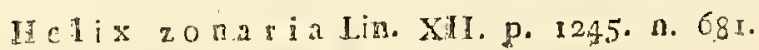

Sctua. 3. t. 4 . f. 53 53. 55

Da Costa. t. 4. f. 7. p. 79. Helix virgata.

Born. Index p. 383. - Test. p. 378.

Chemnitz. Qo p. I40. t. 132. f. Ir88. IIBS.

On ignore fon lieu natal.

9-13. H. c a rocolle, imperforée, convexe des deux cotés: ftrièe, l'ouverture triangulaire.

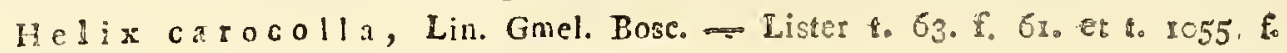
5. Favanne. 4. 63. fo 61. - dans l'Inde.

Is-in. If. cornet militaire, peu carénée, imperforée. convere; l'ouverure évasée, marginée.

Helix cornu-militaris Lin. Bosc. - Gualt. \&. 3. f. r. Ho cornata? Porn. r. I/. f 9. ro. - dans l'Inde. Les citations de Keorr et de Chemnitiz doivent être rayées cltez Gmelin. Elles apparficnnent à l'espéce $\square$. $22,=3$.

18. I H. marginé, ombiliquée, ftriçe obliquement, convexe en dissus, applatie en dessous, le premier tour de spire caréné pouverture triangulaire, transverse, marginée.

If ei ix marginata. Gmelin. Bosc. - Born. t. 14. r. 3. 4. Chemnitz. 9. t. 125 \&. $509 \%$ - On igroe son pays natal.

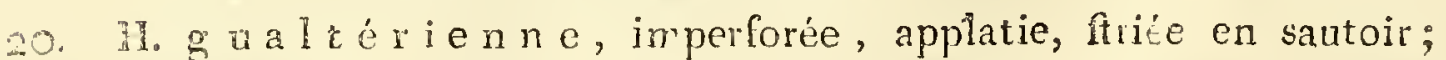
l'ouverture angulense des deux cotés.

Helis gualteriana, Grelin. Bose. - Guals. t. G8. f. E. Born. t. 13. f. 12. i3. Chemnitz. 5. p. $23 \%$ Vign. 44. fo A. B. C. - Se trouve dans Inde. 
2I. H. o ng u li ne, helix ungulina Borm. t. I 5. f. I r. Bosc. 4. 33. Seba. 3. t. 40. f. II. Chemnitz. 9. to I25. f. I098. rogg.

22. 23. H. douteuse, imperforée, applatie, blanche, l'ouverture grande à lèvre reflechie.

Helix dubia mihi. I'ai appellé cette espèse douteuse, parceque (hemnitz, Gmelin et Bosc l'ont donné pour celle, appellée, Corinu militare, par Linné. Cet auteur en citant la figure de Gialtieri ne laisse pas de doute de ce que celle que je viens de dérrire ne soit pas une espèce diftincte. $V$. de très bonnes figures chez Knorr. Del. 6. 1. 32. f. 3. Celles de Chemnitz. 9. t. 129. f. I142.' r143. sont très mediocres. L'un des Individus a 1 pouce 8 ligues de hauteur sur 2 pouces ro. l. de largeur.

* Helices à spire plus ou moins élevéc, à tours convexes.

$24-28$. Heli c e cỉ i ine, convexe, obtuse, jaunâtre, avec une fascie brune.

Hielix citrina Lin. Gmel. Bosc. - Chemnitz. 9. t. I3r. f. rI6. f. rr75. à la Chine et à la Iamaique.

29. H. chat a i g e, presque globuleuse, couleur de chataigne obscure, une fascie rousse, jointe à une blanche, les tours de spire striés.

Helix a stanea, Gmel. Bosc. - Chemnitz. 9. ¿. I3r. f. Irz7. II

30-35. H. des vignes eu escargot, presque ovale, obtuse, fauve sale; l'ouverture demi-ronde.

Helix pomatia. Lin. Gmel, - Chenniz. 9. t. I28. f. Is 38 . Born. t. If. f. 21. 22. Bosc. 4. 27. t. 29. f. 2. - Cans toute 1'Europe.

36. H. I ig a te, presque globuleuse, blanche, avec des fascies rousses et la lèvre blanche.

Hel ix ligata, Gmel. Bose. - Chemnitz. 9. t. I28. f. Ir37' - en Italie.

37. H. ja a ïque, globuleuse, brume, fasciée de blanc; la lèvre decoupée? blanche, le sommet obtus.

Helix jamaicensis, Gmel. Bosc. - Favanne. p. 63. f. M. - de la Iamaïque.

38 - 42. H. pein te, presque globuleuse, unie, avec quatre tours de spire cylindriques, le premier ventru; les autres conprimés, l'ouverture en demi-lune. 
Fie lix.pic:a, Gmel. Basc. Born. Mas. t. 55. f. I7. I8. Chemritz. g t. x30. f. $1162.1165 .-$ cn Italie.

43 - 4. Helices des bos quets, presque ronde, unie, fasciée; l'ouverture alongée, brune.

Helix lucorum, Lino Bosi. Guslt. t r. fo C. Lister. to 105. 8. fo r. da C Ita. to 4. f. rr. - dans l'Europe méridionale.

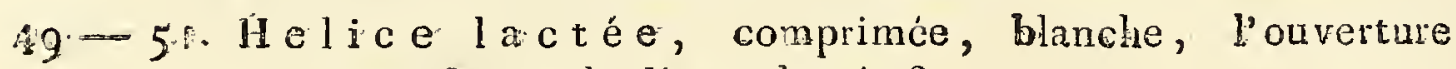
d'un rouge brun; la lévre dentée?

Helix lactea Gmelin. Bosc. Chemnitz. 9. \&. 130. R. 1160 . Se trouve en Portígal et à Ja Famaĩque.

5.2- 76. Hélice des jardins, globuleuse; la lëvre blanche?

Helix hortensis, Gmel. Bosc. chemniz. g. t. 133. f. rig9-120r.

$77-10$. Hélice liviéé, presque ronde, unie, demi-transparente, fasciée, l'ouverture un peu en croissant.

Helix nemorali s Lia. Busc. 4. 45. t. 29 . f. r. Lifter, t. 57. f. 54. Chemgitz. 9. t. r32. f. Irg6. xig7. Li n né n'a adopté que l'espèce tŕelix nemoralis, Müller en a separe le premier, Helix hortensis, mais it parô̂t que ccs deux espèces conflwent ensemble. Je troure du moins en suivant les variations de cinquante exemplaires 'qu'il n'y a pas de caracteres de les diftinquer exactement.

$102-$ I 2 r. Hélice fru it, globuleuse, l'ouvertare à lèrres non bordées.

Helix fruticum. Ginel. Bosc. Schröter Erdconch. to 2, fo 19* Chemnitz. 9. t. 133. f. r203. - dans le nord de l'Allematgne.

* He li c es à spire élevée et pointue.

Nota. Les espéces fuivantes peuvent fournir des caractères pour trois genres difo férens, qui doivent être féparés des Helices.

232. I23. Helix pileus, testa trochiformi alba fasciis rufis, apertura transversa ampla, labro subreflexo. Lim. GmeI. 3637 . n. 89 .

Muller h. verm. 2. p. 80. n. $27 \%$.

Davila. cat. rais. r. p. 445 . n. 996.

Born. Index. p. 390 . Testac. p. 380. to I6. f. 1I. 12.

Schröt. Einal. x. p. 235. n. 208.

Chemnitz. 9. p. 48. to 122. fo 1046 - 10480 
124. Helix papilla, testa conica perforata: ftriis rugosis, apertura transversali; labro reflexo. Müller hist. verm. 2a, p. 100. n. 298.

Helix papilla Gmel,-3660. no 137. Hélice mameloa. Bosc. 4. 53. Chemnitz. 9. p. 51. to 122. f. I053. 1C54. - fon pays natal eff inconne.

I25. Helix e pistylium, testa sübglobosa, candida anfractibus septem. Müller. hist. Vernı. n. 252. p. 5\%.

Notre csemplaire eft d'une belle grandeur et a 2 cours te fpire; - rr liģnes de haut, et ro l. de large à la base.

Trochus a us ralis, testa terrestri, atua, prymidali, laedi, anfraetibus ocio fubconvexis, apice obruso, basi imperforata convera, lab:o acuto. Chemnix. 9. F. 49. t. 122. f. 1079. 1050 .

Helix ef̧istylium Lin. Gmel. 3630. n. 55. Bose. 4. 29.

Nec trochus nec helix ese fed fui generis. Des Istes de lomerdu Sud.

126. Helix effus a, testa gJobosa, unbilicata: anfractibus centiguis; primo maximo, . apertura oblongo-ovata, limbo intus flavesceute.

Cheminis. 9. : p. T180 t. 229. fo I144. II4E.

Seba Thes. 3. t. 4⿻. f. 3t 5 .

Helix oculus con nunis Gme! 3621 . n. 159 .

Bosc. 4. 9r. H. ceil commun.

127. 128. Heliz g l a l c a, testa rumbilicata subrotunda, acu= minata; labro marginata, apertura owali.

Gmelin. S. N. 35280 n. 43. Bess.4.4. 27 ,

Seba. 3. t. 38. f. 8 .

Knort. Del. 5. to 5 , f. 3.

129. 130. Helix senegalensis. testa fubglóbosa lacide fusca, difincte umbilicata, anfractibus contrariis aperturae labro candidissimo. 
Chemnitz: 9. p. 85. t. 109. f. 917.918.

Les dernières especes n. 126 - 130 pourrone entres dans le gence c y clo= s $t o$ in c de Lamarck.

II e $\mathrm{l}$ c i n e. Helicina $\mathrm{L}$ a $\mathrm{m}$ a r c $\mathrm{k}$.

Coq. fuhglobulcuse, non ombiliquée: Quverture entière, demi-ovale columelle calleuse, comprimée inféricurement. Un opercule:

Animal. inconnn.

I - 5. He li c i n a vest i r i a, testa imperforata, conico-convexa, basi gibboso- callosa, apertura subcordata.

Trochus vestiarius Lin. XII. p. 1230. n. 59t. Lister t. 649 - Gua't. t. 65. f. E. Knorr, De!. 6. t. 22. f. 7 ; 4. t. 21. f. 4. Chemniz. 5. p. 70. t. 166 . fo. 1605. a - ho - dans la mer méditerranée, asiatique, ce de la C'hine.

\section{N. e r i t co Nerita L. i $\mathrm{n} n$ a e $\mathrm{i}$}

Coq. remiglobulcuse, appiatie en dessous, non ombiliquée. Onverture: entière, domironde. Columelle fubtransverse, tranchante, fouvent dentée.

Animbal V. Lam. p. 95. Bosc. 3. p. 26\%. de Roissy. 5. p. 268.

1. 2. Nérite couronne, noire, les tours de la spire: couronnés d'épines.

Nerita corona Lin. Bosco. Chemnitz. Q. to. I24. fo 1083. 1084. -- dans les rivières de l'Inde et de l'Amérique australe.

3. Nérite ratissoire, sillonnée; les sillons ftriés, tuberculeux; les tubercules égaux.

Nerita rad ul a. Lin. Bosc. Born. Testo XVII. 7. 8: Chemnitz. 5. t. Igo. fo 1946. 1947: - dans la mer des Indes.

4. 5. Nérite c or née, finement ftriée, violette, avec des ban. des longitudinales, blanches ou jaunes.

Nerita corne a, Lin. Bosco de Roissy. Dargenville pl. 7. f. M. - dans la mer des Indes. 
6-30. Nérite fluviatile, rugueuse et variée de blanc, de brun, de rouge et de jaune.

Nesita flupiatilis, - Lin. Bosc. de Roissy. Chemnitz. 9. t. I24. f. Ic88. daus les grandes rivières de l'Europe. Elle varie beaucoup, comune le prouvent les exemplaires exposés.

31. 32. Nérit e littorale.

Nerita littoralis, Lin. Bosc. de Roissy. Lister. t. 60\%. f. 39. 40. fur les côtes de la mer d'Europeo

33. 34. Nér i t c z è bre, jaunâtre, lisse, avec des lignes parallèles noires, l'ouverture blanche, semilunaire.

Nerita Zebra Chemnitz. 9. Q. 6\%. t. I24. f. 1080, 108 Ie - des rivières des Incles.

* Nérites à lèrvres dentées. Nérites marines.

35. L'ér ite ondulée.

Neritavidulata Gmel. Bosco Chemnitz 50 to 19r. fo, 1970. I97r. - Se troupe dans linde.

36. 37. Nér it e n o ir e, epaisse, opaque globuleuse, peinte de lignes très noirs; le dedans blanc; la lèvre extérieure unie, $l^{2}$ intérieure rugueusè et tuberculeuse.

Nerita at errima Gmel. Bosc. Chemnitz. 5. t. 192. f. I985. I986.

38. Nérite verte, unie, verte; la lèvre intérieure crénelée: dans son milieu.

Nerit a vir id is. Gmel. Bosc. Schröter Flussconch. t. 5. f. ar. a. b. Plusicurs exemplaires dans une petite joite d'ivoir - Se trouve dans la Méditerranée ct aux Antilles.

39. 40. Nérite vi lo in ie, ovale, unie, jaune avec des; zigzags bruns, la lèvre intérieure denticulée.

Nerita virginea, Lin. Bosc. Dargenville. pl. 2. f. P. - dans les fleures? des deux Indes.

4L - 7r. Nérite polie, unie, le sommet oblitéré, l' une et l'autre lèvre dentée.

Nerita polita, Lin. Bosc; de Roissy. Chemnitz. 5. t. Ig3. f. 201 2014. - des Indes. Elle varie beaucoup. 
72-75. Nerite histrione, sillonnée, ftriée traneversalement; la lèvre intérieulo dentée.

Nerita histrio, Lir. Bose. Chemnitzo 5. t. 190. f. [9+8. I949 et to 191. 5960.196 i.

76. Nérite p lissée, sillonnée, les lèvies dentées, l'extérieure arec des dents coniques et aiguës.

Nerit a plice a ta. oLin. Bosco Borm. Testo t. I $\eta_{0}$ fo I\%. If

7ఛ. 78. Nérite grosse.

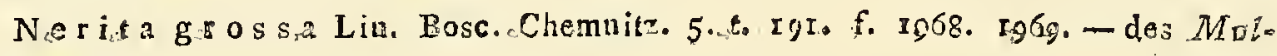
luques.

79. Nérite dé pouillée, sillonnée, les lévres dentées, l'ietérieur seulement tuberculeux.

Nerita exuvia, Lin. Bosc. de Roissy. - Chemnitz. 5. t. 191. f. 1972. 1973. - Se trouve dâns l'Inde.

80. Nérite nègre, très noire, unie, finement ftriée; les lèsres blanches, l'extérieure finement sillonnée, presque dentẻe, l'intérieure concave, rugueuse et taberculeuse.

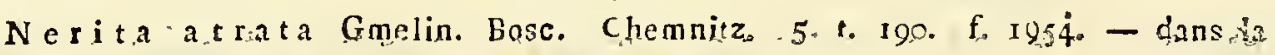
mer atlantique, et dans celle de l'Amérique australe.

81-93. Nérite de plusieurs coule urs, variée de bleu, de rouge, de blanc, aver des fascies rouges et des taches carrées noires; la lèvre intérieure striée, dentée des deux cotës.

Nerita versicolor, Gmelino Bosc. Chemnitz. 5. to Igr. f. 1962. Is.63. Se trouve dans les Antilles.

94. 95. Nérite pie, "blanche, radiée de noir, avec des stries transverses; - la lèvre intérieure rugueuse, à quatre dents? Nerita pica Gmelin. Bosc. Chemuitzo 5. t. 19r. fo r964. 1965n - dans $1 a$ mer de $1 \cdot$ Inlle.

96. 97. Nérite à chotes, pręque globuleuse, enicurée de ftries épaisses, l'intervalle blanc; la lévre dentée des deux côtés, l'extérienre crénelée en dehors; 'l'intérieure convexe, rugneuse tuberculée.

Nerita costat a, Ginel. Basc. Rorn. Test. t. 17. T 19. 20. Chemnitz. 5. 8. 391. f. ay66. ee 1967. - Se rrouve dans la mer des Indées. 
$98-100$. N de quatre coule urs, violette, le bout jaunâtre, le dedans blanc avec des stries élevées, noires; les lèvies dentées de deux cotés, l'extérieure crénelée en dehors, l'intérieure conveze, ruguease, taberculée.

Nerita quadricolor, Gmelin. Bosc. - Chemnitz. 5. t. 19i. fo 19?4. i975. - Se troure dins la ner rouge.

LOI 2. Nérite de mal a ca, sillonnée, jaunâtre en dedans: le sommet élevé; la lèvre extérieure crenelée en dehors; l’intérieure juunâtre, unie.

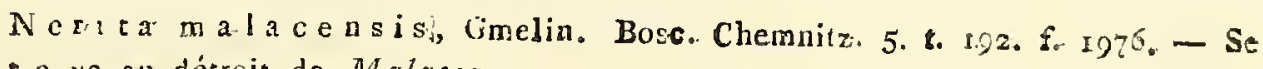
c ouve au détroit de Malacca.

$102-104$. Nérite des antilles, presque globuleuse, noire, sillonnée et striée; le sommet obtus, la lèrre denticulée et rugueuse des deux cotés.

Nerit a Antillax um, Gmelin. Bosc. - Cñemnitz. 5. t. 192. f. 1987. aux Autilles.

$\mathbb{I O S}$ - IO9. Nérite fla mbée; presque globuleuse, avec des stries blanches, transverses, fort rapprochées; des rayons onđulés, pourpre, et la lèvre dentée des deux cotés.

Neri ra fla mmea, Gmelin. Bosc. - Chemnitz. 5. to 192, f. 1992 - 1995. dé la mer des Antilles:

IIO-I14. Nérite foudroyarte。

Nerita fulgarans, Gmelia. Bosc. - Chemnitz. 5. i. 192. f. 1996. 1997. - Sc trouve fur la côte de l'ouest de I'Amérique.

I5. Nérite tour, alternativement fasciée de blanc ou de jaune et de noir; le sommet très saillant, la lévre aigüe; l'intérieur blanc.

Nerita turrita, Gmetin. Bosc, - Chemnitz. 90 to 224. 6. 1085. - dans les caux dauces des Antilles.

116. Nérate bifásciée.

Netita bifa sciata, Gmelin. Bosc - Chemnitz. 5. t. I.93. f. 2015. Se trouve dans la mer des Indes.

I 17. 118. N érite lettrée, presque globuleuse, marqquée de caractères blancs; la lèvre intérieure crénelée et dentée. 
140 NA T I C E. NA T I C A.

Nerita litterata, Gmelin. Bosc. - Chemnitz. 5.t. 193. f. 2016. 2018. Se trouve dans la mer de l'Inde.

119. Nérita pennat a Born Mus. t. I \%. f. 1.1. c'est sans doute une variété de la Nérite polie.

\section{Natice. Natica Adanson:}

Neritae umbilicate Linnae i.

Coq. fubglobuleuse, obiliquéc, à bord gauche caleux vers l'ombilic. Ouverture encière, dermi ronde. La columelle oblique; non dentée.

Arimal. V. Lam. p. 95 . Bosc. 3. p. 285 .

1-3. Natice canrène, unie, la spire un peu pointue; lombilic à apophyse bossue, bifide.

Natica cantena Lin. Rosc. 3. 287. t. 28. f. 5. 6. de Roissy. 5. p. 261 . t. 53. F. II. - Nerita canrena Lin. - Chemnitz. 5. t. 186. f. 1860. 6 t.

4. 5. Deux varié tés d’après Chemnitz. 5. t. I 86́. f. 1862. I863. I'ose croire que ni no. 4. ni no: 5 . peuvent ềrre considérés conme variétés mais doivent former des espèces distinctes à cause de la forme de l'ombilic.

6. 7. Nat i c e grelot, unie, la spire obtuse, l'ombilic à demifermé, la lèvre bossue, bicolore.

Nati c a gla úcina Bosc. de Roissy. - Nerita glaucina Lin. - Chemnitz. 5. t. 186. 1. 1856. 1859. - Dans 12 mer de l'Incle et la Mérliterranée.

8. 9. Natice ja un e d'o e uf, presque globuleuse', l'ombilic entièrement perforẻ.

Natica vitell us Bosc. de Roissy. - Nerita vitellus Gmelin. - Chemnitz. 5. t. 186. f. I866. T867. - daus la mer des Indes.

$10-\mathrm{I} 4$. Natï e mam elon.

N a ti a m a mill a Bosc. - Nerita mammilla Lin. - Chemnitz. 5. t. 189. f. 1923 - 1931 .

I5. Natice fauve:

N.a.tic a fpadice a. Gmelin. Bosc. - Chemnitz. 5. t. 187. f. 1872. I373. t. İ8. f. 1896: a. b. et. I897. - Se trouve fur les côtes de l'îsle de la Réunion. 
16. IV a tice ponctuée, unie, blanche, maculée ct ponctuée très finement de roux; l'apopbyse de l'ombilic bossue et bifide. Natica ftercus muscorum, Gmelin. Bosc. - Chemoitz. 5. t. I87. f. i894. - dans la Méditerrannée et fur les côtes d'Afrique.

1\%. 18. Natic e gochet, presque globuleuse, avec des lignes en zigzags, d'un fauve pâle; lapophyse à fommet applati. Natic a tulm inan s. Bosc. 3. p. 289. Gimelin. - Chemnitz. 5. to I87. f. I88 - fur les côtes d'Afrique.

I9. No toil e d’araignée, blanche, réticulée de roux; l'extrémité noirâtre; les tours de fpire conveses; l'ombilic presque couvert.

Natica arachnoidea Bosco ¿.2९r. - Nerita arachnoidea Gmein. Chemnitz 5. 8. Iss. f. 19i5. IgIG.

20. 2r. Natice melanostome, Mince, demi-transparenie, oblongue, unie; le premier tour de la fpire ventru, très grand, plns comprimé que les autres; l'ombilic presque fermé.

Natica melanostoma, Bose. - Nerita melanost. Grolin. - Chemnitz. 5. t. I89. f. 1926. I927. - Se trouve dans la mer des Indes.

22. Nat-ic e rousse, Roux clair, l'ombilic peu coloré; le bord et la base du dos des fpires fasciés de blanc; la gorge avec une fascie roussattre, et la columelle avec une bande brune.

Na tic a ruf a Bose. 3. 289. - Nerita rufa Gmelin. - Chemnitz. 5. t. 18 ? צ. 1874 , 1875. - fur les côtes de l'îste de la Réunzion.

Hal io i i e. Haliotis. In na e i.

Ceq. applatie, auriforme, à fpire urès basse, presque latérale. Ouverture très ample, plus longue que large, entière. Disque percé de trous disposés fur une ligne pavallèle au bord gauche.

Animal. V. Lam. p. 27. Adans. i. 2. f. т.

I. - 8. II a $1 \mathrm{i}$ o $\mathrm{t}$ id e ormier, presque ovale, le dessus avec des rangées transversales de tubercules rugneux. 


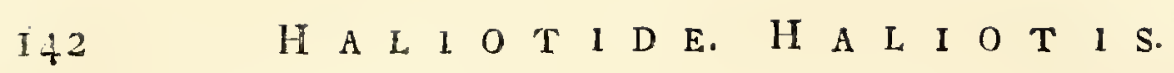

Haliotis tuberculata; Lin. Bosc. 3. 25o. t. 28. f. 7. 8. de Roissy. 5. p. 256. t. 53. f. 10. Martini. r. t. 15. 16. f. 145 - I 49 . C'est l'orcille de mer proprement dite. Deux exemplaires en contiennent de perites ferles, et un troisième est depouillé. - Se trouve fur les cô:es de l'Europe, de l'Afrique et de 1 'Inde.

9-I 4. H a liotide ftriée, ovale, transversalement rugueuse, et longitudiualement ftriée.

Haliotis ftriata, Lin. Bosc. - Matini. r. t. 14. f. 138. - Se trouve fur les côtes Africaines et Afiaíques de la Méditerrannée.

15-22. H. ma r brée.

Haliotis marinorata, Lin. Bosc. de Roissy. Lister t. 6r4. f. 6. Mastini. 1. t. 14. f. 139. - Se rouve fur les cộies d'Afrique et dans l'Imle.

$23-25$. H. a sine, oblongue, unie; le bord en faucille; le dos avec des nervures faillantes.

Ha liotis as inium Lia. Bosc. - Mart. 1. t. I6. f. 550 - dars la rer des Indes.

26. Ha liotide glabre, ovale, unie, verte, variée de blanc; la spire extrêmement petite.

Ha liotis glabra Gnel. Bis: Chemnitz, 10. t 165. f. I602a

27-32. Haliotide ovine.

Haliozis ovina. Chemnitz. ro. p. 3r5. ta 165. fo. 1609. Bosc. 3. p. 253.

33. Haliotide superbe.

Haliotis pulcherrima Ǵmelin. Bosca Chemnitz. ro. to 165. f. 1605.So rouve dans locéan Austral.

34. I a liotide iris, ventrue, d'un fauve clair; hérisé de rides tiansverses et de tubérosités longitudinales; le dedais. brillant des couleurs de l'iris.

Haliotis iris, Gmelin. Bosc. Martyn. 2a t. 6r. Favannea pla 29a f. D lur les cô:es de la Nouvelle Ze lande.

35. Ha li otide c a na liculée, applatie, finement ftriée, la spire applatie, un canal profond au miliea, paralléle: au contour extérieur. 
Haliotis canaliculata mihi. Elle est fetite', brunâtre, tachetéc de blans, cn partic couverte d'une Escare. Le pays natal cn eft inconnu.

36 - 4 . De petites haliotides, dont les espèces ne se laissent point déterminer.

Vermiculaire. Vermicularia $\mathrm{L}$ a marck.

Coq. tubulesse, contournée en fpirale à fon origine, et cntière dans toute fa longueurs Ouvertare fimple et orbiculaire.

Animal. Voy. Lam. p. 97. Adanson t. ir.f. r. Bosc. 5. I55.

1. 2. Vermicula ire 1 o mbrica le, réunie en société; can= nelée en long, et ridée en large; le tube supérieurement droit, et inférieurement à spire aigüe, de 5. à. 12 . tours. $V$ ermicula $\mathrm{r}$ ia $1 \mathrm{umbr}$ ic a lis, Lam. - Vermic vermcus Bose. 5 I55. P1. 5I. f. 3. - Serpula lumbricalis Lin. Lister t. 548. f. I. Gialt. t. Io. f. Q. V. Adarson. pl. I1. f. I. Martini 1. t. 2. f. I2. B. t. 3. f. 24. B. - sc trouve fur les cồles de l'Afrique, de l'Inde ct dè i'Adriatique. Un beau et grand groupe fur une coquille envelopant une baguctic de bos.

3. 4. Vermicula ire massier.

Vermicularia arenaria, Bosc. Serpula arein. Lin. Dargenville pl. 4. f. H. Martini. I. t. 3, f. 19. A. B. C. - fur les côtes d'Afrique et dans la mer des Indes.

5. Vermiculaire calyculée, isolée, cannelée en long, le tube composé d'entonnoirs enchassés les uns dans les autres, l'ouverture évasée.

Vermicularia calyculata mihi.

6-9. Verm i cula i e glomérée, réunie en société, à rídes transversales; tube supérieurement droit, et inférieurement à trois touìs de spire.

Vermicularia glomerata, Bosco - Serpula glomeratct Lin. Gualt. t. 10. f. T. Dargenv. pl. 4. f. G. Marini 1. t. 3. f. 23. - dals la mer da Nord, furl la côte d'Afrique, dàns la Méditerrannée, et mêne dans là Caspieinne.

10? Vermiculaile glomérée, lisse, bleuâtre, avec la ,coquilie crête de coq. 
II. Vermiculaire glomérée, beau groupe avec desspondyles et des serpules.

112. Vermic ula ire glo mérée, beau groupe, entrelacé avec des serpules.

L3. Vermiculaire glomérée groupée fur du bois, avec des balanes et des moules.

14. Vermicula ire glomérée, fossile, avec des porites.

\section{Sili qua ir e. Siliquaria. I. a m a r ck.}

Serpula Li n $\mathrm{n}$ a e i.

Coq. tubuleuse, contournée en fpirale à fon origine, irrégulièrc et divisée latéralemont, fur toute fa longucur, par unc fente etroite.

Alimal. inconnu.

I. Siliquaire ang primés, entrelacés ensemble.

S il iq uaria anguilla Lam p. s8. Bosc. 5. p. 15". Serpula anguilla Lin。

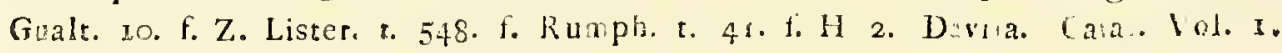
t. 4. f. E. Martini. r. t. 2. f. 13. rq. - Se nouve daus Ja mer des Indes.

2. Siliquaire épincuse, cinq tours de spire, arrondis, en spirale diftante, quatre rangs d’epines écailleuses, plissées.

Siliquaria fpinos a milh. Un exen plaire fuperbe, de trois pouces et domi de longueur, d'un pouce deux ligues de largeus an prerier tour, er de 9 lignes de largenr au dernier lor r. - Serkula echinata Gimel. (3744. 18. H'est pas à confondic arcc notsc esjèce, farcequ'elle n'apparticrt tas neêrie au genre Siliquaria.

3. Siliquaire turbìnée, six tours de spire, arrondis, lisses, en spirale reguliere et contigüe.

Siliquaria turbinat a nihi. Une coquille des plus raes et des plus précieuses, que l'on feroir tencé de prenảre au premier coup d'ocil ponr une fcalaire ou foalate. Les tours le toucheni de fi près, ot le deriter, formant le fommer, feme, comme dans les coquilles hommées, f'onbilic, de forte que l'enreur feroit encore plus pardonable, fi on ne ropoit pas la fente gai caractérise lo geare, e: qui acconpagne les tours jus y. an fommet. Cette ferte parô̂t encose mais presque foudée dans les demiers tours du fommet. Couse 
coquille rare, dont je ne conno:s. pas le pays natal, fait donc gu'll faut rager le mot „irrégulière. de la décription gárique; car la foirale qu'elle forme cat de la plus graude régularité La mêne Siliquaive turbanelle fo trove quelquefois en fociéré, mais cependant isolée, ainsi que l'oa peut ruir, daas le beau groupe de Davila r. t. 4: f. E.

Arrosoir. Penicillás Lam.

Serpula. I i n n a e i.

Coq. tubuleuse, adhé:cnte, rétrécie et un pea en fpitale à fon origine, dilarée en massue vers l'autrc extrénué. Disque terminal correxe, garui de petits tubes perforés.

Animal? parcit appartenir aux-Annelities.

I. Arrosoir de java.

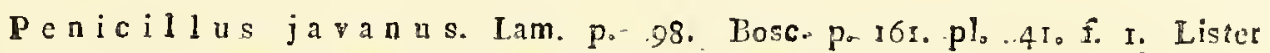
t. 548. f. 3. Gualt t. 30. f. M. D rgenville. t. 3. f. 4. Favanue c. 5. f. B. Martini. I. t. $x$. f. 7 .

2-6. Cinq tuyaus de mer, dont l'un d'un beau volume, ils paroissent appartenir à la classe des Annélides.

Argon a ute. Argonauta Linn a e i.

Coq. univalve, très mince, involute, naviculaire; à fpire rentiant dans l'ouverture. Cárène clorsale double et suberculeuse.

Animal. inconnu.

I - 8. Argona $u$ te papyracée, comprimée, carénée, munie d'un double rang de tubercules coniques.

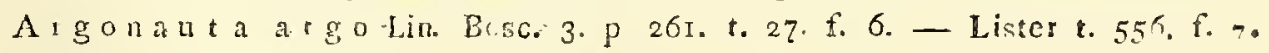
Martini. I. t. 17 f. 157 . - dans la haute mer, en Europe, en Asie, et en Amérique.

L'un des but exemaires est d'une grandeur extraordinaire. Dans quelques uns les côics lout percêes par accidento 


\section{T R O I S I E M E SO US-D I V I S I O N.}

Coquille univalve, multi-loculaire, engainant ou enfermant l'animal.

\section{Na tile. Nautilus Linnaei.}

Coq. en fpirale, fubdiscoide, dont le dernier tour cuveloppe les autres, "et dont les parois font fimples. Loges nombreuses, furmèes par des cloisons transverscs, fimples, et dont le disque est perforé par un tube.

Animal. V. Rumph. Mus. t. r 7 . f. B.

I. 2. Nautile fla mbé, ouverture cordiforme; le. fornmet de la fpire entièrement caché; des fascies brunes en forme de flammes; l'intérieur nacré.

Na usilus pompilius Lin. Lam. p IC0. Bosc. 5. p. I65. r. 42. f. r. Martini. I. t. 19. f. 165. I65. La fpire de l'un des deux exemplaires est ouverte; un exemplaire aacré de la première grandeur fe trouve dans l'armoir où fone exposées des coquilles de grandeur extraordinaire.

Les espèces fossiles Voy. Mus. Demid. Vol. nII. p. 27r. chi l'on trouve aussi décrites les espèces des genres, Ammonites, Discorbites ct autres, appartenans à la même fous-di vision.

\section{Spirule. Spirula Lamarck.}

Coq. partiellement ou complètement en fpirale discoïde, à tours féparés; le dernier furtout f'alongeant en ligne droite. Cloisons stansverses, fimstes, dont le disque est percé par un tube. Ouverture orbiculaire.

Animal, incornu.

I-2. S pirule fragile.

Spirula fragiiis Lam. p. 102. Boss. 5. p. 193. t. 42. f. 2. 3. de Roissy. 5. p. 15. t. 5 r. f. r. Nautilus fpirula Lin, Lister. t. 550. f. 2. Martini. r. t. 20. f. 184. 185 - Se trouve dans les mars d'Amérique et de l'Inde. 


\section{DIVISION SECONDE.}

Mollusques. A céphalés. Première Sous-division.

Mollusques acéphalés nus.

* Il fen trouvent quelques As c i d ies fechées dont il est difficile de déterminer avec exactitude l'espèce.

\section{Seconde Sous-division.}

Mollusques acéphalés conchylifëres。

\section{O $R$ D R E.}

\section{Coquilles équivalves.}

\section{I. $S \&$ c t i o n.}

Coquille équivalve à Charnière farss dents.

Pinn e. Pinna I in na $i$.

Coqo longitudinalé . cunéiforme, pointue à fa base, hâilante en fon bord fupérieur, el fe: fixam par un byssus. Chumère fass dente. Liganent latéal fort longo

Animal. V. Lam. p I12. Basc. 3 p. 173 .

I. 2. Pinnè a $p a n$, fillonée; tes écailles en voûte, disposées par rangées longitudinales.

Pinna r u dis: Lin. Bosc. 3. r-6. Lister. t. 373. f. 2 r $_{4}$. Chemnitz. 8. to 88 .

f. 773. 774. - da.ns les mers des Indes, d'Amérique ct d'Afrique.

3-7. Pinne com an e.

p inna nobilis Lin. Eose. Lister. t. 372 . f. 223 . Chemnilz. 8. t. 8\%. fo 775 - 777. - Se crouve dans liz máulterrannée er fur les cốtes de l'Amérique. Trois camplaires font pacés fur des pieds tournéz, noirs.

8. 9. Pinne papyracée, mince, cornée, fragile, avec des côtes lon itudinales; les bordi arrondis à l'extrémité. 
Pin in a papy acea. Gmelin. Bosc. Chemnitz, 8. t. 23. f. 786 . - Se trouve daus la mer des Indes.

\section{Mo ule. Mytilus Lin. I a m.}

coq. lorgitudinale, à crochets terminaux, droits, faillans, en pointe, et fe fixant par un byssus. Une impression musculaire. Charnière le plus fouvent édentce. Animal. V. Lam. p.. İ3. Bosc. 3. p. 1499.

* Moules ventrues.

I- I 3. Mou le com mu ne, unie, violette; les: valves antérieurement un peu carinées, et postéricurement obtuses; le fommet aigu.

Mytilus edulis, Linné. Lam. Bosc. Lister. t. 362. f. 260. - Chemniz 8. t. 84. f. $75 \circ$. 75 r. 755 . - Se trove dans les: ners de l'Europe es de l'Asie.

14 - I8. Variété, fillonnée, produite par '̂̉art?

19 - 30. Mi oulerong ui lée.

Ir ytilus ungulatus Lin. Bose. Lister. t. 360 . f. 199. ct. 364. f. 203. Chemnitz. 8. t. 84. f. 747 . - Se trouve dans la Méditerrannée es au Cap de bonne Fispérance. L'une est garnie de ferpuics, l'autre de baianes, une troisième est depouillée.

3I. Moule alongée.

Mytilus eloligarus, tesia clongata, ventricosa, margaritifera laevi-, coloribus purpurascentibus, violaceis, caculeis erc. fplendens. Chemnitz. 8, p. I57.t. 83. f. 738. - D'argenville. t. 2 I. f. N. - Moule magellanique Da= vila. Cat. rais. I. 879. - Myo Perno Linnae? - Un exemplaire très opalisant.

$32-38$. Moule africaine.

Mytilus africanus, Chemaiz. 8. t. 83. f. $739-74$. Bosc. 3. 165. Mytilus pictus Born. Mus. t. 7. f. 7. - dans la Méditerrannée, et fur lès cîtes d'Afrique.

39-43. M o ul e b x ulé e, ftrice, le ventre angulecix, le bord crénelé.

Mytilus exustus, Lin. Bosc. Lister. t. 365. f. 205 . Chemnitz. 8." 4.84. f. 754. - Se irouve dans 1.Océan Américain et dans la mer rouge. 
4. Moule percespierre, cylindrique, arrondie à fes extrémités.

Mytilus 1 it hophagus, Linn. Bosc. Lifter. to $42 \%$ f. 2680 et 437 . f. Gualt. t. 90. f. D. Dargenville pl, 26. f. E. Zoomorph. pl. 7. f. 5. Chemnitz. \&. t. 82. f. 729. 330 . - Se tiouve dans ia mediterranée, dans la mer des Indes et dans celle d'Anériquse, perçan les pieras. Sn superbe éehantillon d'un plus giand et d'un plus petit exemplaire, encore enferaé dans un morceau de pierse.

45. Moul e perce-pierre d'une belle grandear de pres. que quatre pouces de longueur fur I 3 lignes de largeur.

46. 4. Mo ule arborisée, fubcylindrique, fragile, d'un jaune aurore, avec des traits bruns, imitant aux bords les desseins d'herborisations.

Mytilus arborescens, Davila. I. to I9 f. z. n. 893. p. 336. Faranne t. 50. f. G Chemnitz. II. p. 25 I. t. I98. f. 20I\%. 2017. Unc moulc très rare de St. Dominique.

49. 49. Mou le variable, oblongue, pyramidale, transparente, transversalement ftriée, a firies concentriques, une bosse variable.

My átre, et a une singulière conformation de ses deux vaives. L'une a une bosse aiongèe aupréz de la charnière, l'autre auprès des bords Celle-ci forme comme une buuche hiarte pour faire sortir le byssus par lequel l' animal s'attache. Cette ouverture des bords cst-clie peut-êttre le caractère d’un genre particulier dont nous ne connoissons que cetre espèce? - Elle fait en rout cas le passage aux Nodioles de $\mathrm{L} a \mathrm{ar}$ a $\mathrm{Ck}$; et $\mathrm{n}^{\prime}$ a rica de commun avec la moule azuscée de Gmelin.

Les moules applaties de Linné vogez plus tard sous le genre Avicule.

Modiole. Modiola I a m a r $\mathrm{k}$.

Mytilus Eir.

Coq. fub-transverse, à cốté pofférieur extrêmement court, ầ crochets abaissés sur le cốté court de la coquille. Une seule impression musculaire. Charnère simple s.uns deat,

Arrimal, inconnu. 
I- 5. Modiole papouane, lisse, finement ftriée, brune rougeâtre。

Modiola pa p a na Lam. po Ir 3 . Mytilus modiolus Lin. Bosc. 3. p 163 . pl. I3. f. I. Lifter. to 356. f. 195. Moule Lulat Adanson. pl. 15 fo 1 Dargenville p1. 22. f. C. Chemuitz. 8. t. 85. f. 757 . et 760 . - Se trouve dans toutes les mers. Un cxemplaire ent garni d'un fucus catyculé; trois autres sont depouillés.

6. Modiole rouge, lisse, mince, transparente, rouge-clair. Modiola $r$ ufa mihï. Ef ce la moule rourge de Bosc? la nốtre n'est pas rugueuse.

7. 8. M od iol e fill lo n née, oblongue; fillonnée longitudinalement et ftriée transversàlement.

9. 10? Modiole gueule de fouris.

Modiola nurina. - Mytilus murinus Bosc. Dargenvo pl. z2. f. K. Sur la côte de Guinée.

\section{An odonte. Anodonte I a m.}

$$
\text { M y t i l a s I i n. }
$$

Coq. transverse, ayant trois impressions musculaires. Charnière simple sans aucune dent. Aninal. Lam. p. I rq. Bosc. 3. I44.

I. Anodonte a nat ine, ovale comprimée, très fragile; le bord membraneux; les fommets rongés.

Anadonte anatina Lam. p. Ira. Boss. 3.146. P1. 23. f. I. Mytilus anatinus Lin. Lif. t. 153. f. 8.

2. 3. Anodonte cygne.

A nodonte cygaea, Bosc. 3. I45. Lia. t. I56.f. F. Chemnitz. 8.f. 762. Mytilus cygneus Lin. La woule des étangs. L'une des valres eft isolée; l'autre est depouillée. 
* Charní̀re à une feu le dent.

Mu lette. Unio Bruguièr e I a mo

M y t i l us $L$ i n.

Coq. transperse, ayant trois impressions musculaires. Une dent cardinale, irréguliére, calleuse, se prolongeañ d'un cò̀é soùs le corcelet et s’aniculaut avec celle de la valve opposée.

Animal. Voy. Lam. p. irt. Buss. 3. 14 r.

I-4. Mulette margaritifère, ovale, le devant plus large, les fonmets rongés.

Unio margaritifera, Brug. Lam. Bosc. 3. 142. - Se trouve dans les lass et les étangs de l'Europe. L'une contient une perîe, et dour en sont depouillées.

5. Mulette depouill é e et nacrée, très belle espèce, grande et voutée.

6. Mulette carolinienne, ovale, alongée, les fommets rongés.

Unio caroliniana Pose. 3. 142. pl. 23. f. 2.

7. Mulette depouillée, presque triangulaire, fillonnée transversalement.

8. 9. Mulette des peintres, ovale, les fommets cntièrs. Unio pictorum. Brugi. Bose 3. 142, pl, 23, f. 3 Lifter. to I 4 6. f. 1. La monle des rivières. - Se trouve dans les eaux douces de l'Eusope.

\section{Mactre. Mactra I.inn.}

Coq. transverse, inéqquilatérale, un peu baillante. Dent cardinale, pliée en gouttière, s'articulane ar celle de la valse opposbe, et accompagnant une fossette qui reçoit le ligament. Une ou deux' dents latérales comprimées et intrantes.

Animal. Voy. Lamarck, p. $12 \mathrm{I}$.

I - 5. Mactre violette, mince, legèrement radiée, finement et transversalement ftriée; le corselet et la lunule blanchâtres. 
152 ISUTRARIA.SOLE

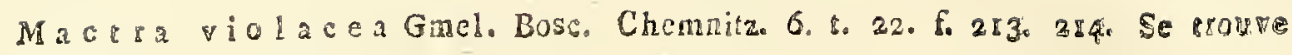
dans la mer des Indes.

$6-10$ ? Mactre ftriée.

Magtra striata Gmel. Busce Chemnitz. 6. 2. 22. f. $22 x_{0}$

* Cladratére à deuz dents。

Lutraire Lutraria Lam a $\mathrm{cck}$.

Mactra Iinnaei.

Cog. transverse inéguilaperate, baillante àx extré nités. Deuz dents cardinales, obliques et divergentes, accoinpag ant une Jarge fusselse gour le ligamens. Dents laièrates nutles.

Animal. incouna.

1. 2. Lutiaire elliptique.

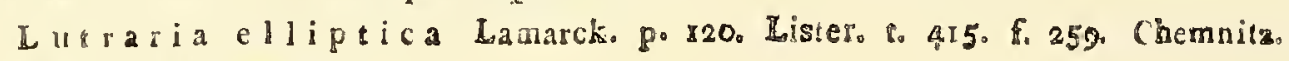
6. 8. 24. f. 24 C. $241 \%$

\section{Solen. Solen Linn a ei.}

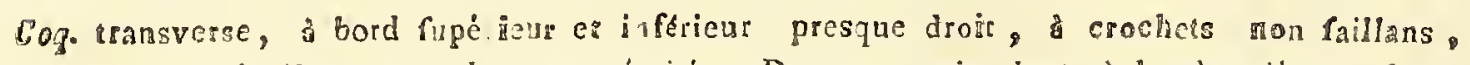
et ba:llante aux deux extrémités. Deura ou trois dents à la claraière, fouro " nies par les deux valyes, Liganent èxtérieur.

Animol, V. Lamarck. po 125. Argenville Zjom. pl. 6. fo Go H.

I - 3. Solen manche de cou e a u; linéaire, droit, l’une des extrémités marginée; la charnière à une faule dent. Sole n ragina Liñ. Lam. Brug ière. Bose, 3. ry. 8. 18. f. 5. Chemnitz. 6. t 4. f. 26. 28. - dane les incrs d'Europe, d'Asie et d'Afrique

4. 5. Solen filique, linéure, droit; la charnière à deus dents d'un côté.

Solen filiqua. Lin. Bose, Chemiatz. 6. to 40 Eo 29. d. - Se trouve dans les mers d'Europe.

6. 7. Solen fabre, linéaire, un peu recourbé: la charnière á deux dents d’un côté.

Solea e as is, Lin, Bosco Chamaitz, 6. \&. 4. fo 30. so 
8. 9. Solen molen d’Adanson; linéaire, ovale, droit: is charnière avec deus dents au milieu de chaque coté, dont une est bifide.

Sole n le gume n Lin. Brugüere. Bosc. Ananson. pl, ro. f. 3. Chemiz. 6 ,

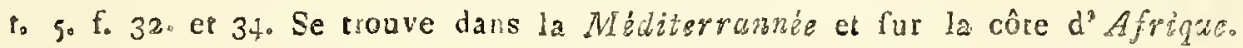

10. Solen de Che mnitz, très petic, blanc, diaphane, fragile, arrondi de deux cotés.

Solen chemnitzii; - 6. p. 48. e. 5. r. 3r. La figure de Chemente devroir être plus alongée. - Se troure Sur les cốtes de Tranquebar.

$11-13$ Solen golar, ovale, obliquement fứé, rongeâter, radié de blanc.

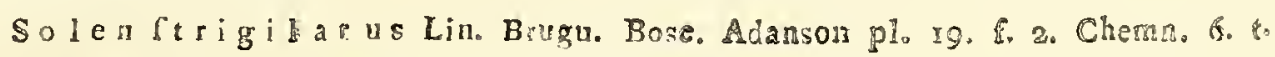
6. fo 4 I - 43. - daus la mer des Indes.

14. I5. Sol e n be c de c a n a rd, ovale, membranerax, velug les côtes de la charnière courbées.

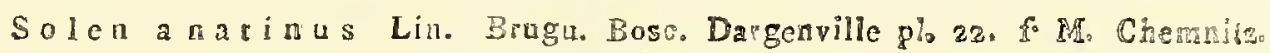
6. t. 6. fo 46. 45. - Se rouve dans la mer des Indes.

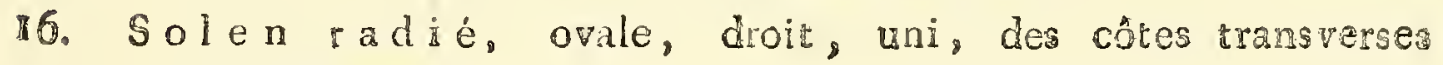
comprimées.

Sole a radiatus. Lino Brugu. Bosco Dargenville pro 22. fo Po Chemirza 6. 4. 5. f. 38 - 40. - Se trouve dans la mer des Indes.

Sang u in o laire. Sanguinolaria $L$ a m.

\section{Solen Linnaei。}

Coq. rransverse, bord fupéricur arçué, un peu baillante aux extrémités. Deux dents crge d:nales rapprochées et ariaculées fur chaque valve。

Animal inconnu.

I - 3. Sa gu inola ire rose.

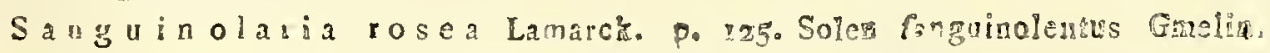
Busc. Chemnitz. 6. ใ. \%. fo 56. - dans da mes des Afritilles.

4-6. Sanguinolaire galano

Sanguinolaria vespertina; - Solen vespesticus emelin Bese

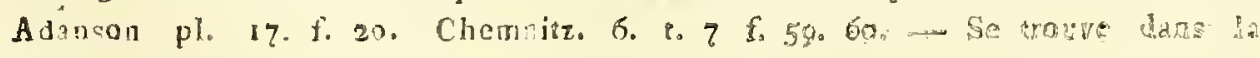
Méditerrannée, à lur la cóte d’ Afrigue 


\section{Capse. Capsa Brugu. La ma rak.}

$C_{\text {oq. }}$ transperse. Daux dents cardinales fur une valve, une dent bifide et intrante fur la value opposée.

Animal incontus.

I- 4. Ca pse rugueuse, ovale, fillonnée, les fillons fimples aux bords.

Capsa rugosa Lamarck. p. I25. - Venus deflorata Linnaci. Chemnitz. 6.t. 9. f. $79-82$.

5. 6. Capse à côtes, ovale, fillonnée, les fillons fe divisant en deux ou trois aux bords.

Capsa costata mihi.

7. 8. Ca p se lisse, fubtriangulaire, lisse; finement ftrièe. Capsa daevis mihi.

\section{T e lline. Tellina Lin n. Ia m.}

Coq. transverse ou orbiculaire, ayant un pii irrégulier fur le côté antéricur. Unc ou deux dents cardinales. Dents latérales écartées.

Animal. V. Lam. p. I24.

\section{* Telli n es ovales comprimées.}

1 - I1. Telline radié e, oblongue, foiblement friée en longueur, luisante; la future de la charnière canaliculée.

Tell i na radiata Lin. Bruga. Bosc. Chemnitz 6. to In, f. Ioo et ro2. Bosc fait, avec Gmclin, double emploi de la dernière figure citée pour $T$. raliata et $\mathrm{T}$. Lanceolata. Se trouve dans les mers d'Europe et d'Amérique. Vulgairement: le foleil levant.

12. Telline violette, oblongue, unie, violette, foiblement rayonnée de blanc, nymphes très alongées et très proéminentes.

Tellina violacea mihi. Une des Tellines les plus rare;, dont point d'autcur a fait mention. Elle eft très grande, de 4 pouces, 2 lignas, de largeur fur 2 pouces de longueur et d' unc couleur violette très funcée. Elle est lisse

- et luisante à l'extétieur et rugueuse à l'intéricur; le pli antérieur est fensible. - des Indes. 
33. Tellìne diphos, ovale, rostrée, violette pâle, fỉlonnée trausversalement.

Te Ilina diphos mihi; - Solen diphos Lin. Bosc. Chemnisz. 6. i. 7 .

f. 53. 5\%. L'exemplatre que $\mathrm{j}^{\prime}$ ai devant moi est peut-êrre le plus grand qui existe, il a precque 5 poures de largenr, et deux pouses et quelques'lignes de lorgueur. - des Incles.

14. Telline vulselle, oblongue, ancérieurement, en forme de bec anguleax; les angles un peu dentés.

Telli na rostrata Lin. Brugu. Buss. Chemutz. 6. t. Io. f. 95. ct. t. Ir f. 104. - Se trouve dans la mer des Indes.

I6. $2 \mathrm{r}$. Tell i n e verge, un angle antérieur; des ftries transverses recourbées; des dents latérales faillantes.

Tellina virgat a. Lit. Brugu. Besc. 3. 19. to I8. f. 3. Dargenville, pl. 22. f. G, - Se trouve dans la mer des Indes.

22. 23. Telline foliacée, corcelet hèrissé, la fente épineuse d'un coté.

Tellina foliacea. Lin, Brugu. Dargentille plo 22. f. E. Chemnitz. 6. t. 10. f. 95. Eosc 3. p. 22. 1. 18. f. 2. - dans la mer des Iniles.

24: 25. Telline de $\mathrm{Kn}$ orr, D'un rouge brillant; le bord violet.

Tellina Knorrii Gmel. Bosc. - Knorr. 5. R. 3r. f. 5o

26. 27. Telline de Born, ftriée transversalement, un des côtés avec un pli rougeâtre; des rayons rouges.

Tell in a Borni i, Gmelin. Bosc. - Borno Mus. t. 2* f. 5. Born l'a appellé angulata, mais is existe déjà une cspèce de ce nom décsite par Linné. La Telline de $B$ orn est très analogue à la virgata $L$ i nnaei; n'est-ce pas un jeune exemplaire de la mêne espèce? -

28-33. Telline i n c a r a te, antériewrement élargie, comprimée, applatie; les fommets un peu pointus.

Tellina incarnata Lin. Brugu. Bosc. Chemniz. 6. to I2. f. I1 $\eta^{\circ}-$ Se trouve dans les mers d' Europe.

* Tellines rondes, plus ou moins convexes.

33. Telline langue de chat, herissée, les écailles en croissant. 
$\$ 5$

Tellina ling ua felis Lin. Brugu. Bosco Chemnitz. 6. 4. 89. 6. 65. - Se trouve dans la mer des Indes.

$34-35$. Telline gargadie, ruguense antèrieurement, la fento dentée.

Tellina gargadiag Lin. Brugu. Bosc. - Chemnitz, 6. t. 8. f. 63. Ct

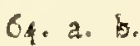

36. Teline ra ge use, des rides transverses ondulées, Is charniére arec deur dents latérales, la principale de la valve gauche divisée en deux.

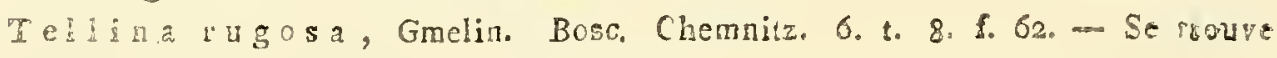
dars la mer de l'Inde et de l'Anérique.

3 - 39? Teline enflée, bossue, blanche avec des ftres longitudinales très fines.

Tellina inflata Gmelin。 Bose. Chemnitz 6. t. 9. f. 76. Est-ce une telline:

40-42. Telline fragile.

Tellina fragilis Gmelin. Boss. Chemnit. 6. t. 9. 6.85. - daus la mes d" Europe et dans ia mer Caspierne.

$43-57$. Tel line de la baltique, unie, incamate.

Telli na baltio a cineh Bosc. 4. 32. Chemnitz. 6. t. I3. fo 128. - dana lia nos Baltique.

\section{Vénus. Venus $\mathbb{L}$ innaei,}

Cog fubotbiculaire on transverse. Trois dents cardinales rapprochées, dont les latérales font plus or moins divergentes.

Animal. V. Lamarck. po r2̧. Bosc. 3. p.

- Venus à épines. Pubrutsos.

I- 8. Vénus di one, presque en coeur; fillonnée ranswers. lement; le tour du corselet épneux.

Ve is us dione. Lin. Bose. 3. 43. pl. 19. f. 2. Chemaitz. 5. 1. 27 . f. 271 . 373. - Se couve dans les mers d"Amérique.

9. IQ. Vénus paphie, presque en coems: des rides épaisses; les environs du corceler avec des rides plus perizes; 1 Jère compliquée. 
Venus Paphia Lin. Dargenville. pl. 2r. f. B. Chemnitz. 6. t. 26. \&. 274. 276. - dans la mer de l' ouest de l'Anérique.

II. Venus marique.

Venus ma ic a Lin. Bose. Chemnitz. 6. t. 27. f. 282 - 286. - dans les mers de l'Amérïque.

12. Vénus dys èré, presque en coeur; des fillons transverses écailleux et slevés; le bord crenelé.

V c nu s d y s e ra Linn. Bosc. Chemnitz. 6. 4. 27. f. $279-285$. dans les mess d'Amérique.

* * V é n us fans épines et presque en coeur.

Impuberes fubcordatae.

I3 - I5. Vénus c lon is se, ftriée par des fillons membraneux, verruqueux, principalement en devant; les bords crénélés. Venus verrucosa, Gmelin. Bosc. 3. 45. pl. Ig. f. 4. 5 . Chemnitz. 6. r. 129. f. 299 a. b. $30 \%$. - dans la Méditerranée, fur les côtes de l'Océan, etc.

16 - 20. Vén us treillisée, des ftries transverses membraneuses écartées; la lunule en coeur.

Venus cancellata Lin. Knorr. 6.t. 39. f. 4. Chemnitz. 6. t. 13. f. 137. 138. Bosc. 3. 46. - Une coquille rate de la mer des Indes.

2 I 22. Vénus poule.

Venus gallin a Lin. Bosc. Cheánitzo 6. t. 30. f. 308 - 310. - dans les mers d'Europe et d'Amérique.

23. Vénus de Guinẻe.

Venus guin e en is Gmelin. Chemnitz. 6. t. 30. f. 3II. et. t. 3 t. f. 3 t2。 Born. MIus. t. 4. f. 8. Bosc. 3. p. $4^{6 .}$ - fur les côtes d' Afrique.

24. 25. Venus cornue.

Venus flexuosa. Gmelin. Bosc. Chemnilz. 6. t. 3r. f. 333 334. - dans les mers des Intes et de l'Amérique.

26-28. Vénus erycine, transversalement et parallèlement fillonnée; les fillons très obtus; le corcelet uni; la lunule ovale.

Veuus erycina, Gmelin. Buse. Chemnitz. 6. t. 32. f. $337-339 .-$ Se trouve dans la mer des Indes. 
29-3r. Vénus me rcenaire, folice transverse, unie, legèrement ftriée; le bord crénelé, le dedans violet, la lunule ovale.

Venus mercenariat, Ling Bosco Chemnitz. In. it. If. f. 16:9. 1660. dans les mes du Nord de l'Europe et de l'A mérique.

$32-33 . \mathrm{V}$ én us chio ne, presque rugueuse transversalement, les dents cardinates postérieures lancéolées.

Venus chione. Lin. B ss. Chemnitz, 6. t. 32. f. 343 . Elle varie beacolng contme le prouvent les exemplaires exposés dont quelquesuns iont depou liés. L'un est garni de ferpulcs. - Sc trouve daus les mers d'Europe, a'Asie, et d'Amérique.

$39-42$ V én us chione, variété tachetée, très belle, figurée par Chemnitz. 6. t. 33. f. 344 .

La figure de Chemnitz $n$ 'aiteint poins nos originaux ni en grandeur ni en coloris.

43-45. V én as des c a m ps, triangulaire, arrondie, bossue, tris unie, avec des lignes en zigzago

Venus. castrensis, Lia. Bost. Chemuilz. 6. to 35. \&. 36\%-38. Se trouve dans la mer des Indes.

$48-5 \mathrm{t}$. Vénus méróc, ovale applatie, frriée trasversalement la Cuture postérieure baillante.

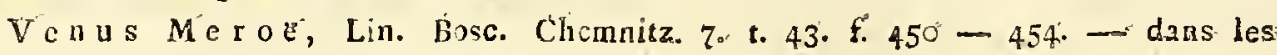

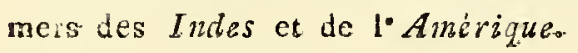

52. Vénus petite:

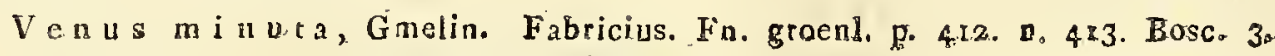
49. - da:is la mer du Nord de l'Eiurope.

53. 54. Véu us réticulée.

Ven us reticula ta Lill. Boss. Brugu. Chemnitz.. 6. ะ. 36. f. 382. 384. dans la mer des Indes.

55. 56. V é n us t i v'e I, presque triangulaire, arie antériearement et obtuse postérieurement:

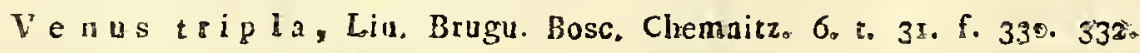

57. 59. Vénús corbeille. 
Venue orbicula Gmelin. Bosc. Chemnitz. 6. t. 3i. f. 326 - Se troure dans l'Océan d'Ainérique.

59-62. Vé nus fran, a is,e.

Venus gallus Gmelin. Bosc. Chcmnitz. 6. t. 32. f. 324.325 . - dans la mer des Indes.

63. 64. Vén us rois ine:

Venus a ffin is Gmelin. Bosc. 3. 54. Chemnitz.=6. t, 33. f. :353. 354. daas la mer des Indes.

65-68. V érus chaste, wlanche, antérieurement convexe, et violette en dedans; le corcelet presque rond; la lunule ovale, d'un violet obscur; le pord entier.

Venus c a s12, Gmelia. Bosc. Chemnitz. 6. to 33. f. 353. 354. - daus la mer des Tndes.

$6 g-74$. Vén us ridée.

Venus corrugata Gmelin. Bosco Chemnitz. 7. to.42. fo 444 . Elle a le port de Capsa de Lamarck, mais les caractèręs de Venus. - Se trouve dans - la mer méditerranée.

75. 76. Vén us tis su e.

$V$ en us te xtil e. Gmelin. Lister, Knorr. Chemnitz. 7. t. 42 . f. $442.443 .-$ dans la iner des Indes.

77. 78. Vénus à trois rayons.

ve us tir adiat a. Gmein. Bosc. Chemniz. 6. t. 34. f. 358.

79. 80. V é n us gé a n t e, ovale, livide; des rayors nombreux bleus et interrompus; 'Ia lunule pvale.

Venus gigante a Gmelin. Bose. 3. 69. Favanne pl. 49. f. T. I. Chemnitz. 10. t. I7r. f. r666. - dậs la mer des Indes et danșcelle d'Amériçue. L'exemplazice le plus grand a 5 pouces de largeur, fur 2 pouces 8 lignes de longueur.

8r. 82. Vénus lettrẻe, ovale, antérieurement un peu angtsleuse; des ftries transverses ondulées.

Venusticterata. Lin. Bosc. 3. 73 pl. 19. f. I. Dargenv, pl. 2 I. f. A. Chemnitz. 7. t. 4r. f. $432-434$. dans la mer des Indes. 
83. 84. Vt́n us écrite, en forme de lentille comprimée, ftriée formant postérieurement un angle droit.

Venus feripta. Lin. Bosc. Chemnitz. To to 40, fo 4200 426. - dans. 1a mer des Indes.

85. Venus crénelée;

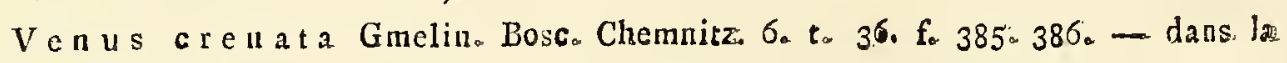
mer des Indes.

\section{Vénéricarde. Venericardia L a m。}

Coq. fuborbiculairc, inéquilatérale, munic de côtes longitudinales. à l'extérieuro Deux Animal inconnu. deuts cardinales épaisses, obliques non divergentes.

I - 4. Vénér ic arde i mbriquée.

Venericardia imbricata Lam. p. 123. Chemnitzo 6. to 30. f. $3140315 \%$ Venus imbricara Bosc. 3. p. 52. Nos exemplaires ne fone pas fossiles?'

5. Vénéricarde frangée, ovale, bossue, ftriée longitudinalement; le bord crénelé.

Venericardia fimtriata mihi. Venus fimbriata Gmelino Bosc. Clemnitz. 7o to 43. f. 448. 449. - Se trouve dans la mer des Indes.

6 - 8. Vé néricarde a c couché e, presque rondes, des ftries membraneuses en fautoir, les bords du corcelet avec des angles faillan's et rentrans.

Venericardia puerpera mihio - Venus puerpera Gmelin. Bose. Chemnitz. 6. to 36. f. 388. 389. - Se trouve dans laz mer des Indes.

L u c in e. Lucina La marck.

Coq. fuborbiculaire ou transverse, n'ayant point de pli isrégulier fur le cốté antérieuro Dents. cardinales variabics. Deux dents laterales écartées.

Animal, incounu.

I- 8. Lucine de jamaĩque,

Lucina jamaicensis Lamarck. p. r34. Pectunculus Lister. t. 300. fo 137. Tellina Klein Meth. S. 40. 11. 14. p. 362. Venus jamaicensis Chemnitz. 7. p. 24. t. 39. f. 408. 409. quclques excmplaires font depouillés. Vulgairen. Game Raffranée. 
22- I 4. Lucine rezeau.

L u c ina reticulat a mihi; - Venus tigerina, Lin. Darganvillé t. $2 \mathrm{r}_{\text {e }}$ f E. Adanson t. 16. f. 3. le Codock; - Knorr. DeI. 4. t. 14. f. 4. Chemnitz. 7. p. 6. t. 37. f. 3 \%o. 39 r. Bosc. 3. 60. ple rg. f. 3. Vulgairement : rezeau blanc; langue de tigte.

\section{Mérétrice. Meretrix I a m:}

Eoqu fubtransverse ou orbiculaire. Trois dents cardinales rapprochées, et une dent isolée, rituée fous la lanule.

Animal. Voy, Lam. p. 122 .

1-3. Méretric e labiée.

Meretrix labiosa Lamarck. p. I22. - Venus meretrin Lig. Bosc. Chemnitz. 6. t. 33. f. 34\%. 348. - Vulgairement la gourgiandine. Les trois exemplaires présentent trois variétés dont la plus rare est celle avee les lignes brunâtres que F a va nn e d e Montce rivelle to 47. f. E. a figuré, et qui paruît former une espèce distincte。

Donace. Donax Linna ei.

Coq. transverse, inéquilatérale, à ligament extérieur, Deux dents cardinales fur lâ valve gauche, et une ou deux dents latèrales écartées fur chaque valve.

Animat. V. Lam. p. I22. Bosc. 3. p. 79.

1 - 4. Donace pamet, antérieurement rugueuse; bossue'; les. bords crénelés.

Don ax rugosa Liu. Lam. Adauson. t. 18. fo I. Pamet; Boss. 3. E०. plo 14. f. 4. Clemnitz. 6. t. 25. f. 250. - Se trouve dans la méditerrannée fur les côtes d'Afrique ot d' Amérique.

5-10. Don a c e gafet, antérieurement unie, violette en dedans, les bords crénelés.

Don ax trunculus. Lin. Gafet. Adanson pl, 18. f. 2。 Chemnitz. 6. t. 26 。 f. 253 . 254 - Bosc. 3. p. 8 r. - dans les mers et fur les côtes d'Afrique.

1I. Donace épineuse, blanchâtre; le dedans bleuâtre, postérieurement unie, et ftriée perpendiculairement; antéri- 
eurement tronquée, et délicatement treillisée; les angles un peu épineux.

Dona x rpinosa, Gmelin. Bosco Chemnitz. 6. t. 26. fo 250 - dans la mer des Tncles.

12. Don ace cuir; triangulaire; en forme de coeur, le corcelet applati.

Dou a fcortum Lin. Bosc Chemnitz. 6. t. 125. f. 242. 2470

13. Don a ce frangée, blanche, mince, le corceler renflé au milieu s à borḍs frangés.

Do n ax fimbriata mihi. Une espèce rare et nouvelle des Indes, quï mérite un examen particulier. C'est une des especes intermédiaires qu"on fera olitigé d'élever au titre de genre, aussitôt qu'il f'en trouvera une feconde.

\section{Ga la thé e. Galathea I a m a r ck.}

Coq. fubtrigone, regulière. Deux dents cardinales rapprochées fur la valve droite avec une cavité ell devant. Deux dents écartées fur la value ganche, et en devant une grosse callosité intermédiaire, fillonnée; nymphes proéminentes,; ligamẹn extéricur très b mbé.

I. Galatété à râyons.

Ga la r he a $r$ d i at a Lamack Annales du Muséum. Tom. “V: P. $430-44$. Pl. 28. Venus paradox Born Mus. p. 66. t. 4. f. 12. 13.

\section{Trida c ne. "'Tridacna Brugui ère.}

6oq. inéquilatérale, fubtransverse. Charaière à deux dents eomprimécs et intrantes. Luñie baillante.

Animal inconnu.

¿- 4. Tr i d a c ne g éa n te, plissée avec des écailles imbriquées et en vonte.

Tridacn a gigas Broguière. Lamarck. p. rrz. Posc. 3. p. 92. pl. 2I. f. 6. Chama Gigas Lin. Cliennitz. 7. 1 49. fo:492.496. 49\%- - dans la mer des Indes ct dans la Méditerrannée. Une feule espèce constitue cc genre jusgu'̉ présent, il paroît ccpendant qu'ily en a de d fférentes, d’opsèc le nombre des fillons á écailles imtriquéeso Parmi les exemplaires de cette collecion il s'en 
3.- Trouve un de grandeur médiocre, lequel présente cinq fillons complets; les autres au contraire quoique beaucoup plus grands $n$ 'en ont que quatse.

H i p pope. Iippopus $\mathbf{L}$ a m a r k。

Coq. inéquilatérale, fubtransuerse. Charnière à deux dents comprimées et intrantes. Lunule pleine.

Animal. inconuu.

1. Hippope tacheté.

Hip popus maculatus Iam. p. rI\%. Basc. 3. po 90. pl. 2r. t. 3. Une fante typographigtre trés grussière de la planche citée dans l'ourage de B osc qui l'est aussi gliscé dans le texte, fair paroitre une confusion des genres Tridacne et Hippope que ce Naturaliste célèbre n'a certainement pas voulu établis. Chama hippopus Lin. Chemnitz. 7. t. 50. f. 498. 499. Vulgairement: le chou ou la feuilie dé chour. - Se rrouve dans la mer des Indes.

\section{C a r dite: Cardita La marck.}

Eoq. inéquilatérale. Charnière à deux denrs inégales, dont lune courre, situées fous les cro:hets et une longitudinale, fe proiongeant fous corcelet .

Animal. inconnu.

I - 2. Cardite variée, fillonnée, blanche, tachetée de brun. Ca id ita variegata Lamarck. p. II8. Chama calyculata Lin. Cheminitz. 7. 5 5. f. 5 o. $50 \mathrm{r}$.

3. C a r dite ínterompue, fillonnée à fillons interrompus, d'un rouge clair.

Cardita interupta mihr.

Elie parrit voisine de la Cardite Ieson d"Adansou pi. 15. f. 8. Cardite calysulata de Bos:- 3. 88. pl. 2I. f. 5.

\section{I s o c a r de. Isocardia I a m.}

Coq. cordiforme, à crochets écartés, unilatéraux, roulés et divergens. Deux dents cardinales applazies es intranies; une demt latérale isolce, fituke fous le corceles.

Animal, inconnu.

- 5. Is oc a rde coe ur, en farme de coeur, presque globuleuse et lisse; les fommets écartés et courbés en arrière en forme de fpirale. 
Is ocardia g lobosa Lamark. p. Ir8. - Chama Cor Lin. Cardita sor

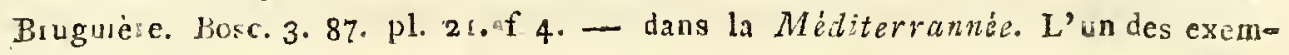
plares est garni d'huitres et de ferpales; un autre avec un mollusque an et feche.

B u c a r e. Cardium I, i n. L m.

Coq. fubrordiforme, à valves dentées ou plissées en leur bord. Charniére à quatre dents, dont deux cardinales rapprochées et obliques fur chaque valve, s'articulant en croix avec leurs corréspondantes. Dẻnts latérilcs écarlées ct intuntes.

4nimal. Vuy. Kéaumur. Adanson Lamarck. p. rig. Bcsc.3. 95.

I. 2. Bucarde coeur de Venus, très comprimée fur les deux faces, les dos des valves carinés, les fommets courbés en dedans, fe couvrant l'un l'autre.

Cardium cardissa Lin. Favanne. pl. 5r. f. P. 2. Chemnitz. 6. t. 14. f 143 - 148 - dans la mer des Indes. Deux exemplaires de belle giandeur, le ples grand, très épinerx aux bords, a presgue deux pouces de longueur, en mesurant la future, et autant de largeur lia oủ les deux valves font les plus Jarges.

3. Bucarde foufflet, presque triangulaire; carinée aux bords de la face antérieure, marquée de fillons ponctués; une lunvile ovale et plate.

Cardium he injcardium Lin. Brugu, Bosc. Chemnitz. 6. t. I6. f, 39. $56 \mathrm{r}$.

4-6. Bucarde marbrẻe;

C a r di um medium Lin, Brugu. Bosc. Chemnitz. 6. t. I5. fo r62. 16я. dans l'Inde et en Amérique. Vulgarement Pigeon.

7. Bucarde arbouse; marquée d'un angle droit au bas dé la face antérieure; les cốtes garnies de ftries saillantes, transverses et colorées.

C ardium unedo, Lin. Brugu. Bosc. Chemnitz. 6. t. 16. f. I68. I69. dans less Indes orientales et aux Antilles.

8. 9. Buc arde é pi n e use, cordiforme, face antérieure alongée les côtes convexes, marquées d'une ftrie au milieu et garnies d'épines courbées en avant. 
Cardium aculeatum Lin. Brugu. Bose, Chemnitz 6. t. I5. f. I56. dans les mers d'Europe.

I0. I I. B u c a rde hérissée, ovale, presque équilatérale; les côtes nombreuses; plates et garnies de beancoup d'épines coudées en avant.

Cardium e chinatum Lina. Brugu. Bose. Chemnitz. 6. t. I5. f. 157.dans la mer des Indes.

I2 - 14. Bucarde tuilée, bombée, en forme de coeur, les côtes garnies d'écailles droites et creuses.

Cardium Isocardia Lin. Brugu. Bocc. Chemmiz 6. r. I7. for I740 I $76 .-$ dans la mer des Indes et aux Antilles.

$15-17$. Bucarde frangée.

Cardi um ciliatum Gmelin. Bosc. Chemnitz 6. t. 17. f. I7r. I72. dans les mers d'Europe.

18. Bucarde fourd on, presque ronde, rustiquée; ringt fix côtes garnies de rides transverses, tuilées à rebour.

C a rdium ed u le Lin. Brugu. Bosc. Chemnatz. 5. to 19. fo. I94. - fur les côtes d'Europe.

I9-21. B u c a r d e pe cti n ée, bombée, marquée de fries longitudinales en avant, et de rides transverses en arrière; les fommets lisses.

Card i um pectinat ưm Lin. Brugu. Bose. Chemnitz. 6. t. I8. f. 18\%. I88.

22. Bucarde exotique.

Cardium costatu m Lin. Brugu. Kaman Adanson. pl. I8 f. 2. Chemnitz. 6. t. 15. f. 15I. 152. Bosc. 3. 193. 11. 16. f. 2. 3. - Se trouve fur la côte de Guinée.

23. 24. Bucarde à côtes $l$ isses, finement réticulées par des lignes ondulées.

Cardium reticulatum mihi. An Cardium magnum Lin. et C. lencostomum Bornil?

* Char nière multidentée ou pectinée.

Pétoncle, Pectunculus $L$ a ma r ck.

Coq. orbiculaire, fubéquilatérale. Charnière en ligne courbe, garnie de dents nombreuses, fériales obliques, articulées ou intranses. Ligament extéricur.

Animal inconnu. 
I. Pétoncle fans oreilles.

Pectunculus ribauritus Lam. p. ri5. Arca pectunculus Linn. Pecten Dargenville. t. 24. f. B. Kaorr. Del. 5. t. I2. f. 4. Chemuitz. 7. p. 235. t. 58. f. 568. 569. Vulg. Peigne ou Pétoncle fans Oreilles. - dans la mer rouge.

2-3. Pétoncle marbré.

Pectunculus mar inoratus m. Arca marmorata Chemnitz, 7. p. 228.

t. 57. f. 563. - des Indes.

4-6. P'ét o n cle velu, presque orbiculaire, équilatéral, velu, et brun; formmets crochus; bords crénelés.

Pectunculus pilosus m. Arca pilosa Lin. Brugu. Bosc. Favanne. pl. 53. f. D. 2. D. 5. Chemnitz. 7. to 57. f. 565. 566. - Se trouve dans la $M \dot{\varepsilon}-$ diterrannée.

\section{Arche. Arca. Lin n. Lam.}

Coq. transverse, inéquilatérale à crochets écarrís. Charnière en ligue droite, fimple aux extrémités et garnie de denis nombrcuses, fériales, transverses, parallèlcs et intrantes. Ligament extéricur.

Animal inconnu.

I - 9. A r he de $\mathrm{N}$ óe, transversale, oblongue, rhomboïdale, et ftriée; fommets très-écartés et crochus; bords fimples et baillans.

A $\mathbf{r}$ a $\mathrm{N}$ a a Lin. Lam. Brugu. Bosc. Dargenville pl. 23, f. G. Favanne pl. 5. f. D. 4. Basbut Gell. Verm. 2. t. 7. f. 3. Chemnitz. 7. t. 53 . f. 529. 531 . Deux individus font foudés ensemble; un autre est placé fur une moule de Papous (Modiolus); un rroisième porte des Vermiculaires; - dans la Méditerrannée, daus les mers des Indes et d'Amérique.

10. II. Arche nacelle;

Arca navieula r is Gmelin. Bosc. Chemnitz. 7. t. 53. f. 533. - dans la mer des Antilles.

12. Arche tailée.

Area imbricata, Gmelin. Bose. Chemuitz. 7. t. 57. f. 532. - dans la mer des Indes. - Chemnitz la regarde comine une vanté de l'Arche de Noé.

I3. Arche de IIelbling. 


\section{A R C A. P H O L A S.}

A rca Helblingii Gmelin. Bosc. Chemnitz. 7. t. 55. p. 542. - dans la mer des Indes et lur les côtes de Guinée. Elle étoit placée fur l'Arche anadara qui f'en est detachée.

$14-16$. A r c he an a d a ra.

A-r $c$ a a n ti quat a, Linn. Bosc. Chemnitz. 7. t. 45. f. 548. 540. - dans la mer des Indes et fur les côtes d'Afrique.

I7. 18. Arche comprimée.

Arca complanata, Gmlin. Bosc. Chemuitz. ?. ‘. 35. f. 54 \% 545 Se trouve fur la cóte de Madagascar. L'un des exemplaires cft furmonté de vermiculaires et d'éponges.

I9. 20. Arche rhomboï de.

A r a rhombea Bornii Mus. p. 76. Test. p. 90 . Knorr. Del. 4. t. 14 f. 2 .

21. Arche inéquivalve.

Arca ina quiva $1 v$ is, Gmelin. Bose. Chemnitz. 7. t. 156. f. $55^{2}$. - de la mer des Indes.

22. Arche $\mathrm{b}$ a $\mathrm{rbue}$, transverse, oblongue; valves applaties et barbues; fommets ferrés; bords fimples (et fermés?)

A r c a b a r b a a cimelin. Bosc. 3. I29. p. 22. f. I. 2. Chemniz. 2. t. 54. f. 535. - fur les côtes d' Europe et dans la ner rouge.

23. A r che radiée, transverse, oblongue, bombée, radiée de fillons doubles, les bords crénelés et fermés.

Arca $r$ a di a ta mihi. Une belle arche de trois pouces fix lignes de largeur, de deux pouccs de hautenr et d'un pouce deux lignes d'épaisseur a la parte la plus bombée. Elle est finement ftriée en dedans, a ftrics éievées et les cuenelures font une impression triangulaire. J'ignore le pays naral.

24. 25. Arche ridée, en forme de coeur; valves marquées de douze côtes lisses et obtuses; fommets courbés en arrière; bords plissés.

Arca fe n ilis Linn. Pose. Adanson pl. 18. f. 5. Chemnitz. 7. 1. 5؟ f. $554-$ 555. - fur la côte d'Afrique.

* à Ch a r n i è r e avec des parties additionelles.

$$
\text { Pholade. Pholas I, in na e i. }
$$

Coq. transverse, jäillante, et composéc de deux grandes valves principales, avec plusicurs perites pièces accessoires placées fur le ligament ou la chanière.

Animal. V. Lam. p. I2\%. Bosc. 2. I95. 
I. 2. Pholade à côtes, ovale, ftriée, avec des côtes élevées.

pholas costata Lin. Lam. p. r27. Bosc. 2. r99. - Chemnitz. 8. t. ror.

f. 863. - dans les mers d'Amérique.

3." Pholade dactyle, oblongue, reticulée par des ftries ritgueuses.

Pholas daceylus Lin. Bose. 2. r98, fl. 8. f. I. 2. 3. Chemnitz. 8. to Ior. f. 859. Se troure fur les côtes des mess d'Europe.

\section{DEUXIEMES S O US DIVISION.}

$$
\text { Coquille inéquivalve. }
$$

Ses valves principales sont inégales entr' elles.

* à Char n i è r e fans dents.

A c a rde. Acardo Brugui ère.

Coq. composée de deux valves applaties presque égales, n'ayant ni charnière ni ligament, une impression musculaire au centre des valves.

* A c ardo umbella.

La marck pense que la coquille que nous avons placéc parmi les patelles, fous le nom de chinvise, vulgairement, parasol chinois, appartienne au genre Acarde. V. n. 117. des patelles.

\section{M a rtea $u$. Malleus $I$ a m.}

Coq. libre, un peu bâillante près de fes crochets, fe fixant par un tyssus, ê ayant fes valves de même grandeur. Charnière fans dent, un peu calleuse, et munie pour le ligament d'une fossette conique, posée obliquement fur le bord de chaque valve, et feparée de l'ouverture qui donne passage au byssus.

Animal inconnu.

I. 2. Marteau vulgaire, à valves égales, ̀̀ trois lobes; les lobes transverses. 


$$
\text { I A L L E U S. A L E }
$$

Malleus vulgaris Lam. p. 133. Bosc. 3. 290. pl. 12. f. 2. Chemniz 8. г. 70. f. 655. 656. Avicula Bruguière; Ostrea Lin. - dans la mer des Indes. L'un est de grandeur considérable et brunâtre; l'autre est blanc.

3. Marteau valselle, demi-transparent, aigu, alongé, fragile, lamellé; une des extrémités arrondie; le dedans uni laisant.

Malleus valsellus Bosc. 2. 290. Chemnitz. 8. t. 70. f. 657. - cals la mer rouge.

4. Marteau anatin, opaque, lamellé, latéralement recourbé.

Malle us a natinus. Bosc. 2. 290. Chemnitz. 8. t. 70. f. 658. t. 710 f. 659. - de la mer des Indes.

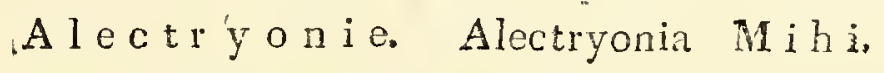

Coq adhèrente, inéquivale, à bords fortement plissés, mais d'égale longicur. Charrière. fans dents. Une fossette carcinale, rriangulatre, fillonnée en trafess, donnant atiache au ligament.

Animal inconnu. La coquille crête de coq a été placée par quelques Naturalistes parmi les Moules par d'autres parmi les Huitres. Elle doit furmer un genre particulicr, fuivant les caractères exposés.

I. A lectryo nie rare, les plis des bords lisses, peu fréquens très profonds, rarement épineux d'un coté, des épines de la charnière fervant d'attache.

A le ceryonia rara, Mihi. Mytilus crista galli Lin. Born. Bosc. Ostrea Kleill. Chemaitz. 8. p. 52. t. 75. f. 683. 684. - Se trouve dans la mer aes Indes. Vu'gairm. Créte de coq, Oreille de cochon, Aile de Chauve-Souris.

2. Aletryonie parasite, des plis fréquens peu prófonds écailleux, tonte la valve inférieure, fervant d'attache.

Alectryonia parasiticamihi.

3. A lectryonie hyotide, des plis des bords fréquens éca. illeux, à écailles épineuses de deux cotés, un endroit glabre noduleux auprés de la chanière fervant $d^{\prime}$ dttache. 
Alectryonia hyotis mihi; - Ostrea hyotis Chemnitz 8. p. 58. t. 75. f. 685. - Mytilus hyotis Lin. Born. Bosc.

4. Alectryonie feuille, en forme de feuilles, les plis fréquens et lisses, un double rang d'épines fervant d'attache.

Ale ctryonia frons. mihi. Ostrea frons Chemnitz. 8. p. 6I. t. 75. f. 686. Lister. Gualtieri. D'argenville. Mytilus Liu. Born Bosc. Vulg la feuille, das Kammblatt. Un groupe de plusicurs individus attaché à une baguetre d'Antipathes. 11 n'y a qu' une qui est encore bivalve.

\section{Huître. Ostrea Lin. L a m.}

Coq. adliérente, inéquiralve, à bords des valves fimples ou plissés, inégaux ( $l$ un dépassant l'autre). (harnière fans dents. Une fossetic cardinale oblongue, fillonnée en travers, donnant artache au ligament. Une feule inpression musculaile dans chaque valve.

Animal. V. Lamarck. p. 132. Boss. 2. p. 296.

I. Huitre concentrique alongée, ovale, applatie, la valve fupérieure avec des couclies concentriques, I'inférieure dépassant la fupérieure de beaucoup.

Ostrea concentrica mihi. Elle est attachéc à un balane, qu'elle elltoure à motié, et toute la valve iuférieure a éré atrachée.

3. Huit re chinoise, Inégale, rugueuse ou lamellée; plus creuse, et terminée par un prolongement.

Ostrea fincnsis Gmel. Bosc. Chemnitz. 8. t. 72. f. 67r. a. b. c. - Se trouve dans la mer rouge.

3. H. corne d'abondance, valve fupérieure applatie; inférieure, convexe, écailleuse, plissée, et ridée, 'fe terminant par un prolongement.

Ostrea corn ucopiac Gmelin. Bosc. Chemnitz. 8. t. 74. f. 679. - dans la mer des Indes et d'Afrique.

4? Huitre papyracé e, presque ronde, mince, demi-transparente, blanche; la valve fupérieure terminée par un prolongement court et aigu. 


\section{OS TRE A. A V I C U L A.}

Ostrea papyracea Schioler Einl, ind. Conch. 3. p. 378. Gmelin. 3337. n. I21. Bosc. 2. 305 . - dans la mer du Nord et dans celles des Intes.

5. Huitre ga i n, la valve fupérieure emboitée dans l'infé. rieure qui a un bord très élevè.

Ostrea capsa, mihi; - Adanson. p. 200. pl. 14. f. 2.

6-8. Hu itre gazar, mince, la valve inférieure convexe, plus épaisse; l'autre applatie.

Ostrea parasitica. Gmelin. Bosc. Adanson pl. I4. f. O. Chernitz. 8 t. 74. f. 681. en Afrique et dans 1'Inde.

Avicule. Avícula Lam a r ck.

Mytilus I, in.

Coq libre, un peu bâullante vers fes crochets, se fixant par un byssus, et avant fes valves d'inégale grandeur. Charnière fans dent, un peu calle: se. Fossette du ligament oblongue, marginale et parallèle au bord qui la foutient.

Animat, inconnu.

I - 4. Avi c u le hi rondelle, applatie, oblongue, lisse, la base transverse à aile trìs longue.

Avicula co $m \mathbf{m}$ u i is Lam. p.134. Mytilus hirundo Lin. Lister. t. 22c. Dargenville. t. 19. f. B. Knorr De!. 4. t. 8. f. 5 ; t 5 i. 10. f. I. 2. le chanon d'Adanson. t. 15. f. 6. Chemnitz 8. p. 136. t. 81. f. 722 .

5 - [3. Avicule perlifère, applatie, presque orbiculaire, la base transverse à aile très courte, imbriquée de lames dentées.

Avicula ta argaritifera, Bruguière. Bosc. Dict. d'hist. no Mytilus margaritiferus Lin. Bosc. Coq. 3. 160 . pl. 13. f. 4. 5. Lister. t. 22 I. f. 56. Chemnitz. 8. . f. $7^{17}-720$. Plusieurs excinp'aires ont conservés leurs lames épineuses, d'autres font intéréssans par leur grandeur. Elle fe troure das la mer des Indes et dans celle d'Amérique, et produit la plus grande partie des perles que l'on roit dars le commerce.

14.- - 16. Trois valves isolées de l'avicule perlifére, dont deux ornées de caracteres chinois et la troisième deponillée. 
Perles. orientales isoléés.

* Paragone ou perle orientale très grosse, trouée à la base; d'un pouc e de longueur, et de $d \mathrm{i} x$ lignes de diamètre, à l'endroit le plus gros.

* Une loupe ou coque de perle d'un pouce de longueur et de $\mathrm{d}$ i $\mathrm{x}$ lignes de largeur.

* Un bouton de perle tourné.

* Hu it b a roques isolées de grandeur différente, et inférièures aux autres.

* Quatre petites perles de la Russie qui font très belles.

* Perle alongée et artificielle, collée et préparée de nacre de perle.

* Plumes de paon, ou nacre de perle changeant, deux morceaux arrondis.

\section{Peign e. Pecten Brugui ère.}

\section{Ostrea Linnaei.}

Coq. auriculée inéquivalve, à crochets contigus. Charnière fans dents. Ligament intéricur, fixé dans une fossene triangulaire et cardinale.

Animal. V. Lam. p. 135. Bosc. 2. p 257.

I - 6. Peigne gigantesque; des rayons arrondis et ftriés longitudinalement.

Pccten maximns Lam. p. 155. Tosc. 2. p. 259. Ostrea maxima Lin. Chemnitz. 7. t. 60. f. 585 - 587. - dans routes les mers d'Europe.

7-i3. Peigne de S. Jac ques, quatorze rayons anguleux, longitudinalement ftriés. 


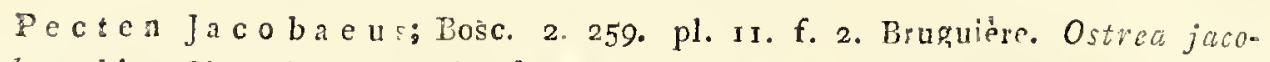
baea Lin. Chemuilz. 7. เ. 60. f. 588. 589. - dans la Mediterrance, et sul: les côies de Portugat.

14. Peigne zigzag, feize rayons applatis. Pectenzigzag Buguière, Dose; - Ostrea zigzag Lin. Chemnitz. 7. t. 6 I. f. 592. 592. - daas la mer d'Amérique.

15. 16. Peigne sole, valves égales, douze rayons doubles, les extérieurs uris.

Pecten pleuronectes Bosc. Ostrea pleuronactes Lin. Chemnilz. 7. to f. 5\%5. Des meis des Irdes.

17 - I9. Peigne de I.aurenti.

Pecten Laurentii Bosc. Ostrea Gmelin. Chemnitz. 7. t. 6r. f. $593-$ fur les côtes d'Anuérique feptentrionale.

20. Peigne du J a po n.

Pecten japonicus Bose. Ostrea Gmelin. Chemnitz. 7. t. 62, f. 596. fur les cûtes d'Afrique et fur colles da Fapon.

2I-26. Peigne ratissoir.

Pecte a radulus. Bose. 2. 26r. fl. 3. f. Ir Ostrea radula Lin. Chemnitz. 7. t. 63. f. 599. 600. - dans la mer des Indes.

2\%. 28. Peigne ratissoir, variété depoúillée.

29. Peigne manteau.

Pecten pallium. Brsc. 2. 267. Ostrea pallizan Lin. Chemnitz. 7. 4. 640

f. $60 \%$ - Se trouve aux Indes.

$30-35$. Peigne gl a bre.

Pccten g laber Chemnitz. 7. p. 338. t. 67. f. 641 - 645. Ostrea glabra Liı. - Dans la mer alriatique.

$36-39$ ? Peigne ro uge, glabre, rouge.

Pccren ruber, Bosc. 2. 266. Regenfuss. I. t. 9. f. 34 .

* Peignes à orcilles inégales.

40. 43. Peigne noueux, neuf rayons, avec des noeuds vésiculaires.

Pecten nodosu.s. Bosc. 2. 268. p'. II. f. 4. Ostrea nodosa Lin. Chemnitz. 7. t. '4. f. 6co. 611. cans l'Océan d'Afrique et d'Amérique.

$44-50$. P e igne d'is l ande; 
Pecten is 1 andicus Busc. Ostrea islandica Gal. Chemnitz. 7. s. -65. f. 615.6r6. - dans les mers du Nort.

$5 \mathrm{I}-56$. Peig ne oblitéré, uni, vingt.quatre rayons doubles. Pecten obliteratus Bese. 2. 268 Ostrea ob.iterata Gmel. Chemniz. 7. t. 66. f. 622. 624, - Se trouve dans la mer des Julles. L'un des exempiaircs porte des V'ermiculaires, l'autre le tuyeau a' we Sabelle.

57 - 59. Peigne demi-nu.

Pecten feminudus. Busc. 2. $27 z$ Ostrea Seminula Gmel. Knorr. Del 6. t. 9. f. 4 .

60-64. "? Peigne blessé.

Pe cte n sa uciatus. Bosc. Ostrea Sauciata Gmelin. Chemnitz. 7. t. 69. f. H. - Se rrouve dans la mer ronge. Queiques uas ont des épines sur les fillons et paroissent former une espéce nouvcile.

65. Peigne fanguinolent, à valves égales, neuf rayors épais et obtus; les intervalles ftriés longitadinalement, noueux et épineux.

Pecten fanguinolentus, Bo:c. 2. 26\%. Ostrea fanguinolenta Gmelin. Chemnitz. 7. t. 64. fo 608. - dans la mer rouge.

66-68. Peig n e c it rin, orangé, vingt deux rayons arrondis; le bord plissé, la voute applatie.

Pecten. eitrinus Busc. Ostrea citrina Gmelin; Chmnitz, 7. t. 65. fo 6r8. - fe trouve dans la nier des Indes.

69. Peigne fanguin.

Pecten ranguineus. Bosc. 2. 26o. Ostrea sanguimea. Gmelin. Chomnitz. 7. t. 66. f. 628. - dans la Méditerraneé, et les mers d'Afróque et d'Amésique.

70- 72. Peigne da Tranque bar.

pecten tranquebaricus Bo:c. 2. 273. Ostrea tranquebarica Ginclin. Chemnitz. 7. t. 67. f. 647.648. - dans la mer de 1'Itule.

73. 74. P e igne varié.

Pecten varius Bosc. 2. 269. Ostrea varia Gmelin. Chemnitz 7. t. 65. f. 633. et 634. - dans la Méditerranée.

75. Peigne histrionique, mince, applati, demi-transparent, avec des rugosités fines. 


\section{P E C T $\mathrm{T} E$ N. $\mathrm{L}$ I $M$ A.}

Pecten histrionicus. Bose. 2. 2-c. Ostrea hisirionica Ginelin. Chemnitz. 7. t. 65 . f. 614 .

76. Peigne imbriqué, à valves presque égales, applaties, avec neuf rayons inégaux, chargés d'écailles tuilées.

Pecten imbrieatus Buse, 2. 262 Ostrea imbricata Galin. Chemnitz.

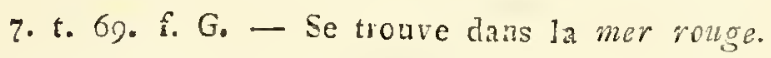

77. 78. Peigne fillonné.

Pecten fulcatus Bosc. 2. 270. Ostrea fullcata Gmel. Chomnitz. 7. t. 63. f. 603. 604. - daus la mer des Indes.

79-88. P eigne e n fant, à valves égales, quarante rayons filiformes, une feule oreille.

Pecten pusio Bosc. 2. 269. Ostrea pusio Lin. Chemnitz. ?. t. 67. f. 635. 636. - Se trouve dans la Méditerranée.

89-98. ? Peigne en fant, variété, rouge tachetée.

L. im e. Lima Bruguière

Ostrea Lin.

Coq. inéquilatérale, auriculée, un peu bâillante d'un cớe entre les valres. Charnière fans dent; ligament extérieur; crochets écartés。

Animal. Voy. Lam. p. 136.

I. L i me exc a vée, blanche, folide, des ftries longitudinales onduleuses, avec quelques anneanx transverses; le bord très-entier.

Lim a exeavat a. Bosc. 2. 253. Ostrea excavata Gmelin. Chemnitz. 7. t. 68.

f. 654. - Se trouve dans la mer du Nord.

2-4. Lime glaciale.

Li ma gla cialis Bosc. 2. 253. Ostrea glacialis Lin. Ostr. aspera Chemnitz.

7. t. 68. f. 652.653. - dans les iners d'Aniérique.

* * à Charnière dentée.

a. à Charnière à une dent.

C a m e. Chana Li n. La m.

Coq. adhérente, inéquivalve, à crochets inéganx. el ayant deur impressions musculaires dans chaque valve. Charnière composíc d'une fetle dent épaisse el oblique.

Animal. V. Lam. p. 131. 
I. Came feuilleté e, couverte de feuilles lâches, tuilées, dechiquetées; les bords legèrement plissés.

Ch ama Lazarus, Lin. Lam. Bosc. Chemnitz. 7. t. 5r. f. $507-509$. dans la mer des Indes. Une icule valve.

2-9. C a m e i m briqué e, couverte de feuillées ferrées, tuilées plissées ou épineuses; le dedans et le bord des valves legèrement ftriés.

C h a m a i mbric a ta Lainarck. p. זзr. Chama gryphoïdes Bosc. Bruguière. Gmelin. Chemnitz. 7. t. 51. f. 5 10. 553. - Se trouve dans la méditerrannée. Quelquesunes font fimples, d'autres groupées, ou ensemble ou avec d'autrs corps marins, tels que coquilles et autres.

IO- 15. C a m e a r c in elle, presque en forme de coeur, garnie de côtes longitudinales, épineuses et de fillons pointillés; les bords des valves crénelés.

Cha ma arcinella. Lin. Brugu. Bosc. Chomnitz. 7. t. 52. f. 522. 523. Se trouve dans les mers d'Amérique.

b. Charnic̀re à deux dents.

$$
\text { Spondyle. Spondylus I. in. }
$$

Coq. inéquivalve, auriculée, hérissée ou rude, et à crochets inégaux, dont l'inférieur plus avancé offe une facetce plane, triangulaire, partagée par un fillon. Chanière composée de deux fortes dents crochues et d'u..e fossette internetdiaire qui donne attache au ligament. Une foule impression musculaire.

Animal. inconnu.

I - Ig. Spondyle ga i d e ron, un peu oreillée, épincuse. Spondylus ga eda ropus Lin. Lam. Erugu. Bose. 2. 315. pl. 15. f. 4. dans la Méditerranée. 11 y a une infinité de rariécés; les bornes qui me font préscrites, ne me permettent pas de décrire ceux que le lectenr trouve réunis ici. No. I8. et 19 font des valkes isolées. Qnelquesunes futment. des grouppes, d'autres font attachées à des coraux.

20. 21. Spondy le gaideron, variété fuperbe, à feuilles très larges et roses.

22 - ag. Spondylus croce us de Chemnitz. $7 \cdot$ t. 45. f. 463. n’elt qua une variété de lá même espèce. 
30. Spondylus nicobaricus da même auteur (7. t. 45. f. 469. 470. est. de même une variété de l'espèce précedente, à épines très fines.

3I. Spondyle royal, fans oreilles et épineuse.

Spondylus redius Lin. Brugu. Bosc. Dargenville. pl. zb. f. G. L. M. Chennitz. 7. t. 4G. f. $47 i_{0}^{\circ}$ - Se trouve dans la Méditerrannée es dans la mes des Indiks.

\section{Pl a c un e. Placuna. B r ug ui è re.}

Coq. libre applatie, a valves de même grandeur. Charnière intérieure offrant sur une valve, deux dents longitudinales ou côres tranchantes, rapprochées par leur extrémiré infétieure, et divergentes en fuite en forme de $\mathrm{V}$; et fur l'autre valve, delix impressions qui correspondent aux côtes cardinales, et donnent attache au ligament。

Animal inconnu.

1. 2. Placune placenta, presque ronde, blanche nacrée; des ftries longitudinales très fines, en sautoir, avec des rides transverses.

PI a cu ua placenta Bruguière Encyclop. pl. 173. f. I. 2. 3. Lam. p. I35. Bise. 2. 27\% pl. 7. f. 5. Anomica placenta Lin. Chemuic. 8. t. 79. f. 7ró. - Vuigairement: la vitre clainoise. - Se trouve dans la mer des Iniles.

2. 4. Plac,un e felle, presque quadrangulaire, convexe, dorée; le bord un peu finueux.

placuna Seli a Bruga. Bosc. Anomia Clemnitz. 8. p. Irr. t. 79 f. 7 r Vulgairement: la felle polonaise, la felle angloise. - Se trouve dans la mer des Indes.

Térébratule. Terebratula. Bruguière.

Coq. régulièrc, fixée par un ligament ou un tube conrt, et compofée de denx valves inégales, dont la plus grande a fon crochct arancé presqu'en bec et percé d'un trou par où passe le ligament. Charnière à deux dents.

Animal V. Lam. p. 139. Bosc. 2. p. 225. 
I- 4. Térébratale vi trée, ovale, ventrue, très mince, transparente, deux rayons osseus à la charnière de la valve inférieure.

Terebratula vierca, Lam. p. I39. Bose. 2. 230. pl. 7. f. 2. Anomia terebratula Litl. Chmnitz. 8. to 78 . f. 707-799. Vulgairem. La poulette. Se rouve dans la Miéditerranée.

5- Io. Térébratule perroquet, conleur de corne, finement et longitudinalement ftriée, la valve la plus courte bossue; la plus grande applatie, le trou triangulaire.

Texebratula psitcacata. Bosc. 2. 230. Dargenville. pl. 23. f. o. Chemnitz

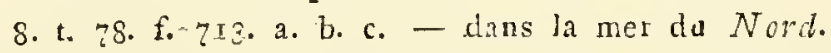

II. "Térébratule crénelée, triangulaire, à valves peu bombées, ftriées, à bords crénelés, le trou rond, et droit, (non caché par le bec.)

Terebratula crenara mili. Fspéce petite mais très belle, qui fetrouve dans une petite bate d'ivoir. J'ignore de quelle mer elie vienne. Elle ressemble a la tronquée de Born, par la Charnière come tonquée, mais en diffëre par sis sillons élevés, par les bords crénelés, et par fa grandeur.

** * Charnière à plusicurs dents.

\section{Perne. Perna Brugui ère.}

Coq. libre applatie, fe fixant par un byssas. Charnière compoée de plusieurs' dents linéaires, parallèles, tronquées, non articulées, et rangées fur une liggne droiıe, transverse ou oblique. Les interstices des dents donnent attache au liganent sur chaque valve.

Animal inconur.

I - 3. Ferne felle du cheval, à valves égales, orbiculaires, comprimées, membraneuses.

Perna ep hippium Brugu. Encyclop. pl. i75. f. 2. Lam. p. I3. Bosc. 2. 283. Ostrea ephippium Lin. Chemnitz. 7. t. 58. f. 576.577. - Se troule daus la mer des Indes. L'un des individus est couvert de valves imparfaites, de coquilles et de vermiculaires.

4. Perne jsogone, à valves égales, le lobe latéral plus long que l'autre. 
Perna isogon a Brugu. Lam. Ostrea isogona: Lin. Chemnitz. 7. t. 59. f. 583 534. Bosc. 2. 283 . Pl. 12. f. 3. - Se trouve dans la mer des Indes et daus celle d'Amérique.

Addition de quelques coquilles dont plusieurs fe distinguent par leur grandeur extraordinaire.

Noła. Les dernières sont numérutécs: ct fe trouvent exposés dans une armoirc particulière. Voluta e. I. 2. Voluta olla. 3. maculata.

* Voluta aurantia, orangée; les quatre premiers tours de fpire avec une fascie blanche. La columelle à quatre plis. Chemnitz. 4. t. I 50. f. I 393. I $394 ; 10$. p. 15 1. Vulgairement le pavillon d"orange. La couleur en est bien vive, Ta fpire entière, boutonnée.

* Voluta hebra i ca, trois exemplaires.

Cassidea e. 4. 5. Cassidea $\mathrm{t}$ u berosa; Euccinum Linnaei. 6. 'a même crensée, de sorte qu' on ne voit que la spire extérieure. 7. 8. C. plicata. $9-16$ cornura 17 . C. reticulata, espèce fossile.

Strom bi. $18-21$. Strombus gigas

Pter o c erae. 22. 23. Pterocera lambis. 2\%. 25. Pt. gallus. 26. 27. 2S. Pt. lucifer. 29. 30. Pt. bryonia. 31. Pt. fossile; elle est casite. 32 - 34. des parties de ftrombus lucifer de Imné. $35-37$. Pt. Cliragra.

Murices. 39. 39. Marex lampas Lin. 40. 4 I. M. reticularis. $42-45$ M. ramosus. 46. 47. M. Tiitonis.

T u r bine 11 us. $47^{b}$ Turbinellus pyrum; exemplaire fossile:

Fusus. 49. Fusus morio; Murex morio, Jin.

Fas ciolaria. 49. 50. Fasciolaria cardoscolym. Chemnitz. IV. p. I6 t. t. I 42 . f. I 325 .

Trochus. 5 I, a. b. Trochus niloticus Lin. 
Turbo. 52. 53. Turbo olearius I. dépouillés et nacrés. B u $l 1$ a. 45. 55. Bulla Achatina Lin. Acbatina Lamarck. Na utilus. 56. Nautilus Pompilius. L.

Pinn a. 57. Pinna rotundata.

* Tridac na gigas d'une grandeur prodigieuse est déjà citée au fecond volume fous le nom de Linné, Chama p. 279 n. 236.237 . où chaque valve a été désignée par un numero particulier.

Hi p p pus. 58. 59. Hippopus maculatus; Chama Lin.

Avicula. 60-6I. Avicula margaritifera; deus exemplaires, de belle grandeur, complèts, - Ostra Lin. $6 \div-64$. Trois valves isolées de la même, dont deux font fculptées.

Astrea. 65. 66. Un beau et grand groupe d'buitres; un autre dans une bouteille cassée.

spondylus. $67-6$; : Trois groupes de fpondyles, Spondylus gaedaropus Lin. 


\section{$\begin{array}{lllll}N & O & T & E & S\end{array}$}

\section{et Explication des Planches.}

I. Les corps naturềs fe diftinguent en
corps organisés et bruts.

2. La condition de l'exiftence des uns eft l'organ is ation, celle des autres s'appèle Affinité.

3. Les uns forment l'idée de l'Individualité, les autres celle de la divisibilité.

4. Il n'y a pas de ligne de démarcation entre les corps organisés, (entre les animaux et les plantes); les Naturaliftes ont don:c vainement cherché une différence entre eux; Hedwig, H u mboldt, Smellie, Ackermann; - Senebier; Ludwig, Us lar, Willden ow, Sprengel, Treviranus.

5. L'auteur adopte dans les áninaux: $x_{0}$ une faculté de reproduire fon femblable dependant d'une feule partie douée d'une crganisation particulière b. un organe particulier et central favo. risant, la circulation des fluides; - et dans les plantes: a. la faculté de reproduire fon fentblable, repandue fur toutes les parties isolées; (racine, tige, branche, feuille, bourgeon, fleur, fenence) c. point d'organe particulier et central favorisant la circulation des fluides.

6. Mais aussi dans cefte observation les plantes paroissent des a nimaux compofés et les poly pes femblent inferieurs aux plantes.

7. Les polypes sopposent à toute division naturelle, même fi l'on ne cher. che dans - les êtres organisés qu'une fimplification d'organes.
8. L'auteur trouve dans la nature organisée une opposition remarquable, qui pourroit être exprimée par feux cercles t. mouve. ment qui fe touchent au qui fe crusuent, en deux endroits:

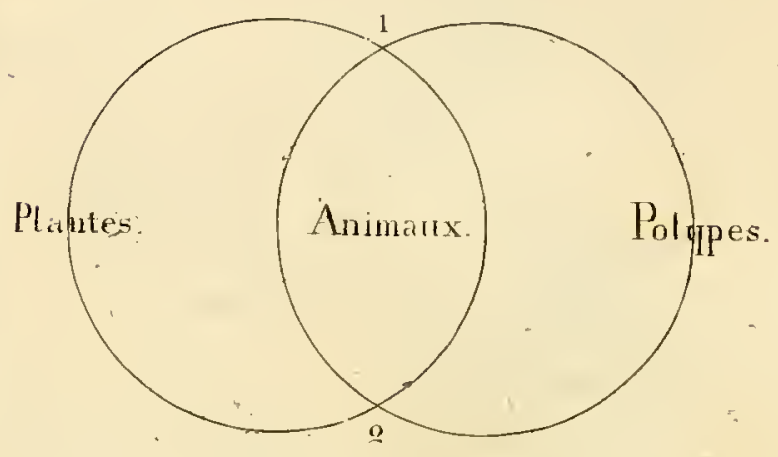

Cetie opposition apparente forme le fyftéme de l'équilibre entre la nature organisée et la brute; les plantes trans. forment la matiere brute; elles la portent au regne organique, l'assinilent: les polypes rendent par un nouvement contraire la matière organifée à la terre. Les tectonourgiens ou polypes coralligènes, ne paroissent exifter que pour transformer la matière animale en. chaux. Les deux points des cercles qui fe touchent, désignent deux ternes extrèmes, deux circonftances inexplicables pour le Naturalifte: I. la mo issisure produite par la corruption des matières aninales; 2. l'origine des anina ux infusoires par celle des matières végétales. - Les bornes de cet ouvrage ne permettent pas un developpement plus ample de cette idée qui préseute une vérité à poursuivre. 
9. Le rapport de la Zoógnosie avec les fciences phyfiques en genéral eft developpé dans le tableau fuivant:

Pliysique universelle, Kosmologie.

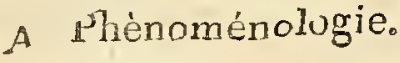

I. Chymie.

a Universelle.

b Particulière.

* Organurgie.

Zourgie.

Phyturgie.

* A norganurgie.

Lithurgie.

Halurgie.

Pyruménurgie.

Metallurgie.

2. Physique expérimertale.

3. Mathématique pure.

4. Mathématique appliquée.

\section{B. Somatologie。}

1. Uranclogie.

II. Géologie.

$\boldsymbol{\alpha}_{0}$ Organologic.

I. Zoologie.

a Zoognosie.

Maftognosie: (Mammifères).

Ornithognosie (Oiseaux)

Amphibiognosie (Reptiles).

Ichthyogriosie (Poissons.)

Malakognosie (Mollusques)

Liothyliognosie(Annélides.) Malakoftracognosie (Crufta. cés.)

Entomogriosie (Insectes.)

A rachnognasie(Arachuides)

Helminih guosis (Vers.)
Afteriognosie (Radiaires.)

Polypognosie (Polypes.)

b. Zootomie.

c. Zoophysiologie.

d. Zoopsychologie.

e. Zoopathologie. sart vétéri-

f. Zoothérapie. $\{$ naire.

2. Phytologie.

a. Phytognosie.

b. Phytotomie.

c. Phytophysiologie.

ß. Anorganologie.

I. Oryctologie; Minéralogie gé-

a. Oryctognosie.

Lithognosie.

Halognosie.

Pyruménognosie.

Metallognosie.

b. Minéralogie chymique.

c. Geognosie.

d. Mineralogie géographique.

e. Minéralogie écononique.

2. Atmosphérologie.

\section{Classes d'Animaux.}

Io. J'ai ajouté des planches pour $\mathbf{r}^{-}$Ies cinq premières classes, pour faire voir la charpente osseuse des quatre premiéres, et la diftance des autres, qui en font dépourvues. "Les planches necessaires à l'explication de la terminologie feront partie de ma Zoologie.

I I. M is non feulement le squélette mais. encore les autres partics lorment une. echelle de degradation d'organes que l. a marck a tiés bien représentée dalls le tableau fuivant: 


\section{TABLEAU DU REGNEANIMAL,}

MONTRANT LA DEGRADATION PROGRESSIVE DES ORGANES SPEGIAUX JUSQU'A LEUR ANEANTISSEMENT.

Nota. La progression de la dégradation n'est nulle part réguliére ou proportionnelle nrais elle existe: dans l'ensemble d'une manière évidente.

Les $\quad\left\{\begin{array}{c}\text { Vivipares et à mamelles; } 4 \text { mera bres ar- } \\ \text { ticu!és déperdaus du queletie. Des pou- }\end{array}\right.$

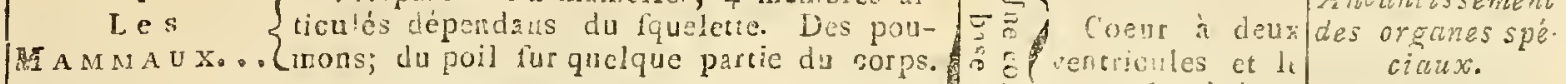
$\mathrm{L}^{2} \mathrm{es} \quad$ Sarticules et sans mamelles; 4 membres Orsea X X.... \{adhéreas; des plumes sur la peau.

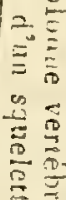
Reptiles... S n tout remps, ou semlement dais le del àge. Ni poils ni plumes sur la pcau.

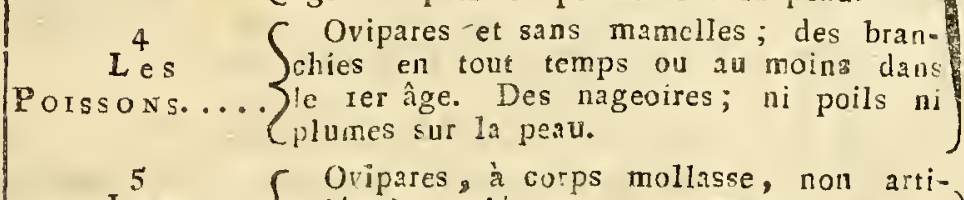
. Les $\{$ culé ni annelé, ayant un manteau variable. Mollusques. Des branchies.

6. C Oripares, à corps mollasse, alongé,

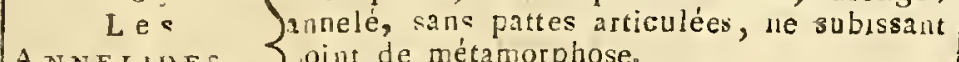
ANNELIDES. $\{$ oint de métamorphose. $z$
Les, $\quad\left\{\begin{array}{c}\text { Ovipares, ayant le corps et les membres } \\ \text { rtcules, a pau crnftacée et ue subissant }\end{array}\right.$ C. Pr T A CES. $\quad\left\{\begin{array}{c}\text { wint de meramurphose. } \\ \text { Dis branchies. }\end{array}\right.$

$8 \int$ Ovipares, ayant en tout temps des patLes $\quad$ es articulées, des yeux a la têtc et ul ARACHNIDES. $\{$ ubissaut point de riétamorphose.

Des stiginates et des trichées.

9 - Ovipares subiscant des métamorphoses, e:

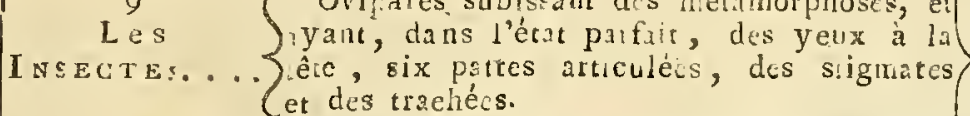
Cet des traehécs.

Io $\int$ Gemmovipares, à corps mou, régénéra-

Les $\quad\{$ if , ne subissant poin de métan., l'ayan

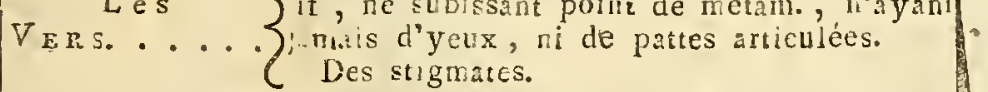

Gemmovipares, a corps régénératif, dé-

ri Spourva de tête, d'yeux, de pattes art., et

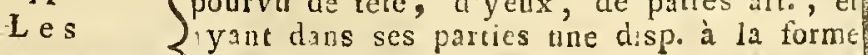
RADIAIRES. . $\left\{\begin{array}{l}\text { yant dans } \\ \text { Donante. }\end{array}\right.$

Das rachées aquifères.

Gemmipares et fis:ipares, à corps presque

I2 $\quad$ généralcment gélatineux, régéuératif, et Les $\quad\left\{\begin{array}{l}\text { nayant aucun organe special in. autre } \\ \text { qu'un canal intestinal à une seule owverture. }\end{array}\right.$

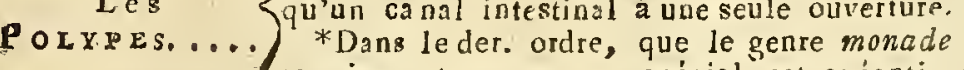
termine, tout organe spécial est atéanti, la génération a’est plus que fissipare. 
I. M a mmifè res.

52. Le fquelette de Didel phe (Tab. I. Didelphis cancrivora, et marfupialis L. Gmel.) présente une particularité que l'on. ne trouve que chez les Didélphes, favoir les os marfoupiaux (ossa marsupialia) ou la fourchette du basin qui fert d'attache à la bourse. Ces animawx nanquent d'ongles aux pous ces des pieds de derrière.

23. L'auteur écrit Quadrimanes comme longimanus etc. Ceux qui disent quadrumanes pensent devoir fuivre l'étymologie de quadrupède.

If. La famille des Tarsiers eft fi prononcée dans la nature, qu'elle s'offre fans developpement a l'observateur.

I5. L'ordre des palmigrades eft dicté par l'anatomie comparée et par an grand nombre de propriétés particulières des animaux qui le composent.

I6. Il ne faut pas croire que la férie des mamnifères foit à considérer dans une direction droite comme une fuite. J'ai fait voir à mes élèves, qu'elle forme une galerie oú l'observateur fe trouve au milieu, ayant les espèces d'animaux de fes côtés. C'eft à dire lauteur s'imagine que chaque férie de la premiére division dont les doigts ou pieds ne font pas réunis par unc membrane, trouvera des analogues parmi les animaux de la feconde division dont ies doigts on pieds font réunis par une membrane. Une représentation des genres de mammifères en cercles entourant Ie centre où eft placé l'homme et fo toushan mutuellement, fuivantque les propriétés de différens animax $f e$ resseinblent, feroit peut- etre la plus conforme à la nature.

\section{II. $O$ is e a u $x$.}

I7. Le fqueletto des oifeaux (Tab. II. l'autour, falco palumbirius) eft déjà moins complèt dars lés extrémités, mais il a un os dé plus auprés de la poitrine que l'on appele fourchotte et un autre au crane qui eft carré et qưi facilite le mouyement de la machoiro inférièurt.
18. L'anteur a fuivi le fyftème de Lacepéde qui eft d'une clarté parfaite. Il a feulement féparé Pipra el Manakina et les a divisús en oxdres très distans, comme la nature l'exige.

\section{R e ptiles.}

r. Le fquelette des reptiles ( Tab. ITI) perd de fa perfection. La grenouille a un flemuin et un busin, mais point de côtes; le ferpent a des côtes très nombreuses mais ni fternum ni basin. Le fquelette de ferpent déssiné d'après nature appartient à un genre à crochets venimeux. (Crotolus) qui font fixés par des parties molles mais que -j'ai taché conserver à la place qu'ils occupent dans l'animal vivant.

\section{Poissons.}

20. Le fquelette de poisson; l'uranoscope (Uranoscopus fcaber). Les: poissons occupent une piace inférieure aux re. ptiles parceque les organes de rérpiration font moins complêts. Ils réfpirent par des branchies et la circulation paroit fimple, c'eft à dire le Sang qui a été poussé par le coeur uniloculaire aux branchies ne retonrne plus au coeur mais fe diftribue, retournant des arcs branchiaux aux différentes parties du corps.

V. Animaux invertébrés.

2x. Tous les animaux des Classes furvantes n'ont ni fquelette, ni colonne verié. brale articulée, ni quelque chose d'azalograe. Lios de la féche, les or des oursins ont toute une autre déftina. tion; quoique la charpente du ventricule des oursins foit formée de véritables os, il faut la considérer comme un prolongenent des dents. Toutes lés autres prarties dures que l'on trouve dans ces classes, tellesque Caquilles, croute des Ciruftacés, des Radiaires, Polypiers, présententun assemblage dé la matiere calcaire, qui, toat regulier gitil efl quelquefois, na rien de commun avec les os qui composent le fquelette des a classes antérieures, 
Mollusques. Tab. I.

22. Pnemoderme de Cuvier. Le genre a été décrit p. 7 .

I. Face antérieure, de grandeur natu. relle.

2. Face poflérieure.

3. Le mème, la tête en bas. Les branchies font placées à l'extrémité opposée du corps et forment deux lignes faillantes en forme de DC adossés.

4. 5. Tritonic arborescens.

6. 7. 3. Scyllaed pelagica (décrit p. 10) par le coté droit, par le ventre, et par le dos.

9. Glancus hexapterygius voy. le genre p. 8.

I0. I I. Eolide. Voy, p. 8. Cuvier avoit déjà indiqué ce genre dans fon Tablecru élément. p. $388 . \quad \mathrm{La}$ marck, Bosc, de Ro ıssy réunissent les especes a'Eolide avec les Tritonits.

\section{M oll usques. Tab. II.}

23. Denominations des parties des coquilles et des animaux qui les habitent.

A. Vis macul e.

B. Strombe oreille de Diane.

C. Natice grelot.

D. Peigne ratissoir.

E. Venus treillissée.

Denominations des parties de ces coguilles.
a. lOuverture, apertura
b. la base. busis.
c. le ventre.
d. le dos.
e. la columelle, columella.
f. la. lèvre droite.
g. la lèvre gauche。
h. l'ombilic.
i. les tours de fpire.
k. le fommet.
1. le bord antérieur.
m. le bord poftérieur.
n. le borá fupérieur.
o. la charnière.
p. le corcelet.
q. la lunule.
r. les oreilles.

F. Anatomie de l'helice des vignes ou du grand escargot fuivant Cuvier。

I. la bouche.

2. le fphincter de la bouche.

3. le premier eftomac.

4. le fecond eftomac.

5. l'intestin grèle.

6. glandes falivaires.

7. lobés du foie.

8. cavité commune de la generation.

9. la matrice.

ro. la glande féminale.

I I. l'ovaire.

I2. l'oviductus.

a. la verge.

b. la bourse du dard.

c. les vaisseaux fibreux.

d. muscle du reservoir de la pourpre.

e. reservoir de la pourpre.

f. les muscles retracteurs du pied.

g. les nuscles transversaux du corpso

h. les muscles retracteurs des cornes.

* " dard rompu.

G Anatomie de l'Anodonte cygne ou de la moule desétangs fuivant Cuvier.

a. Contour de la coquille.

b. Les muscles qui ferment la coquille.

c. Deux lobes triangulaires qui entourent la bouche.

d. Le pied.

e. Les branchies qui contiennent les petits dans leur intérieur.

f. Corps glanduleux blanc que Cuvier croît etre le testicule.

g. Une des oreillettes dans fon état de dilatation.

$h$. Les valvules qui font communiquer avec le coeur.

i. Le coeur.

k. Canal intestinal qui traverse le coeur.

1. Le rectum.

mi. L'anus.

n. Les tentacules du bord postérieur du lobe gauche du manteau.

o. Le reste de ce lobe.

p. Le lieu de l'estomac entouré par lo foie.

r. Circomyolutions du canal intestinal. 
186 Notes et Explication.

24. Noms des Auteurs qui ont étécités dans les tableaux de Zoognosie.

Ad. Adanson.

Bi. \} Bloch.

Br. Bruguière.

Bsc. Bosc.

Cl. Clairville.

Cuv. Cuvier.

D. Daldorff.

Dum. Duméril.

F. Fabricius.

Fisch. Fischer.

F. Forskäl.

Fröl. Frölich.

Fist. Forster.

Fv. Favanne.

Geer. de Geer.

Gfi. Geoffroy.

Gfi. II. Ge offroy St. Hilaire.

Gz. Gäzе.

H. Herbst.

Hg." Hoffmanseeg Comte.
Hlw. Helwig.

Ill. Illiger.

Isn. Knoch.

Kugl. Rugel mann。

L. Linné.

Laich. La icharting.

L. C. La Cepéde.

Lm. Lamarck.

Lath. Latham.

Ltr. Latreille.

M. Müller. O. IF

Ol. Ollivier.

P. Pallas.

Per, Percn.

Pk. Paykull.

Prf. Preyfsler.

R. Römer.

Rud. Rudolphi.

Sch. Schaffer.

St. Steven.

IV. TValkenaar.

Wb. Weber. 


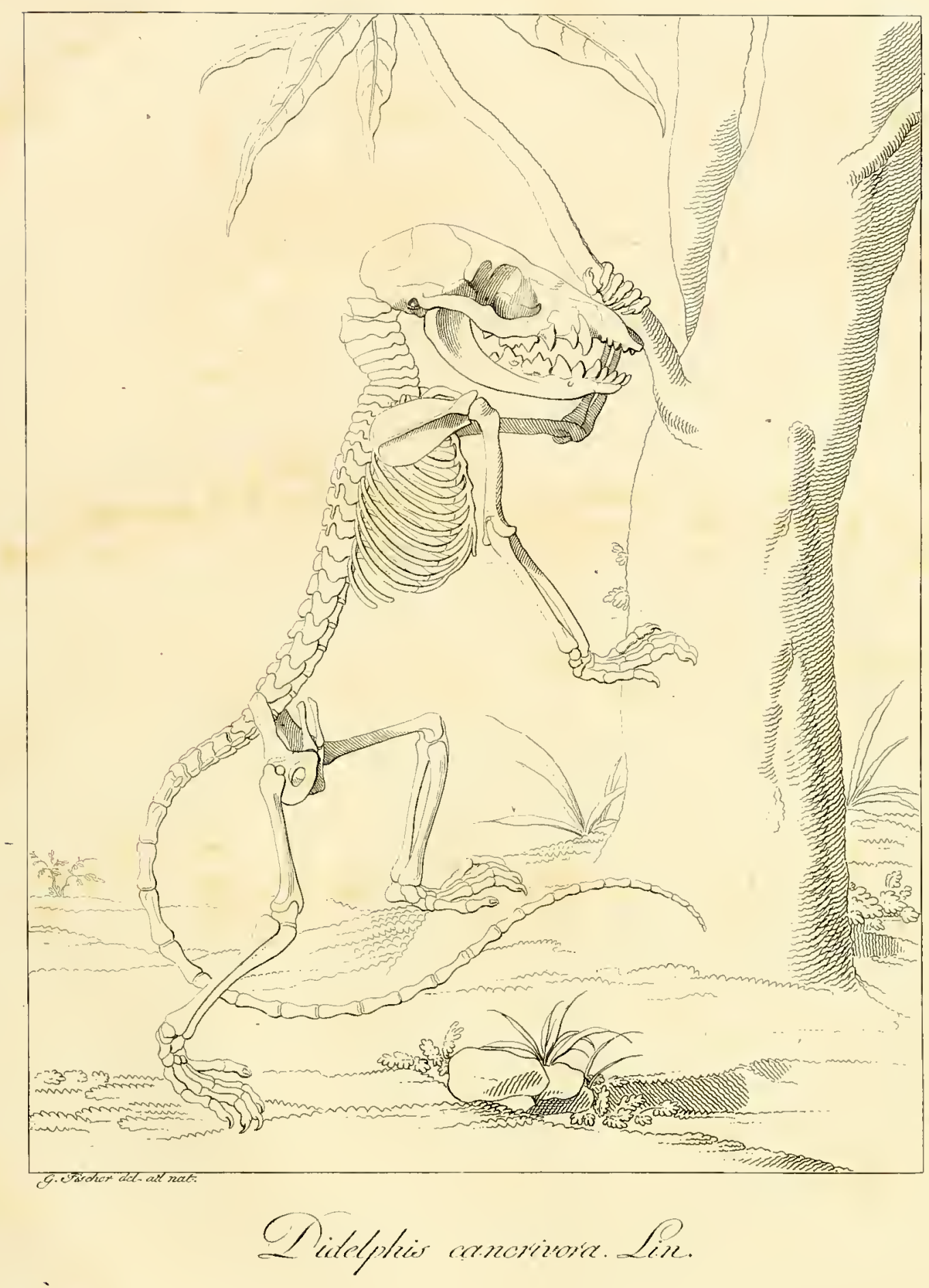




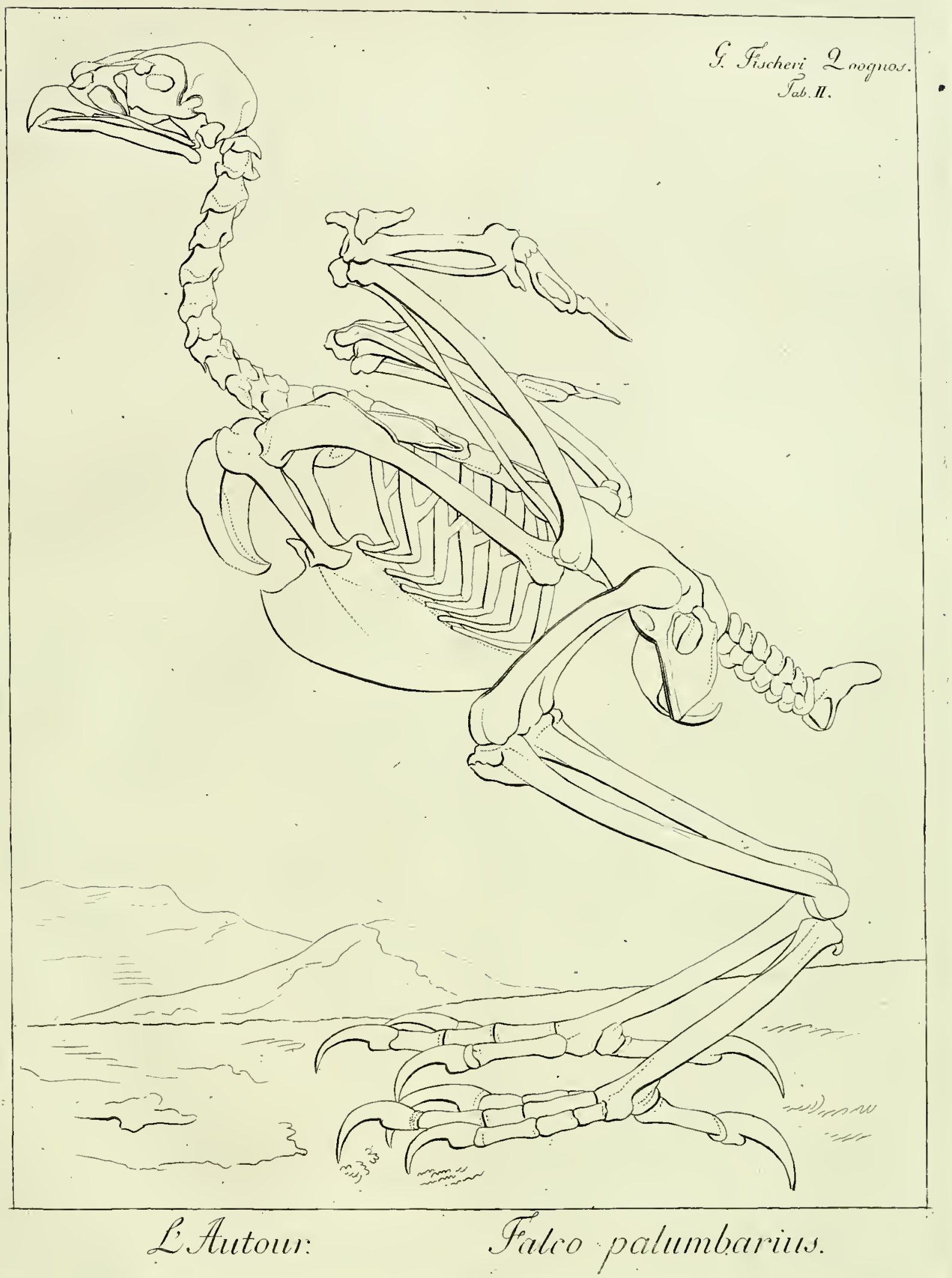




$$
\text { , }
$$


Fischeri Zoognosia.

屈

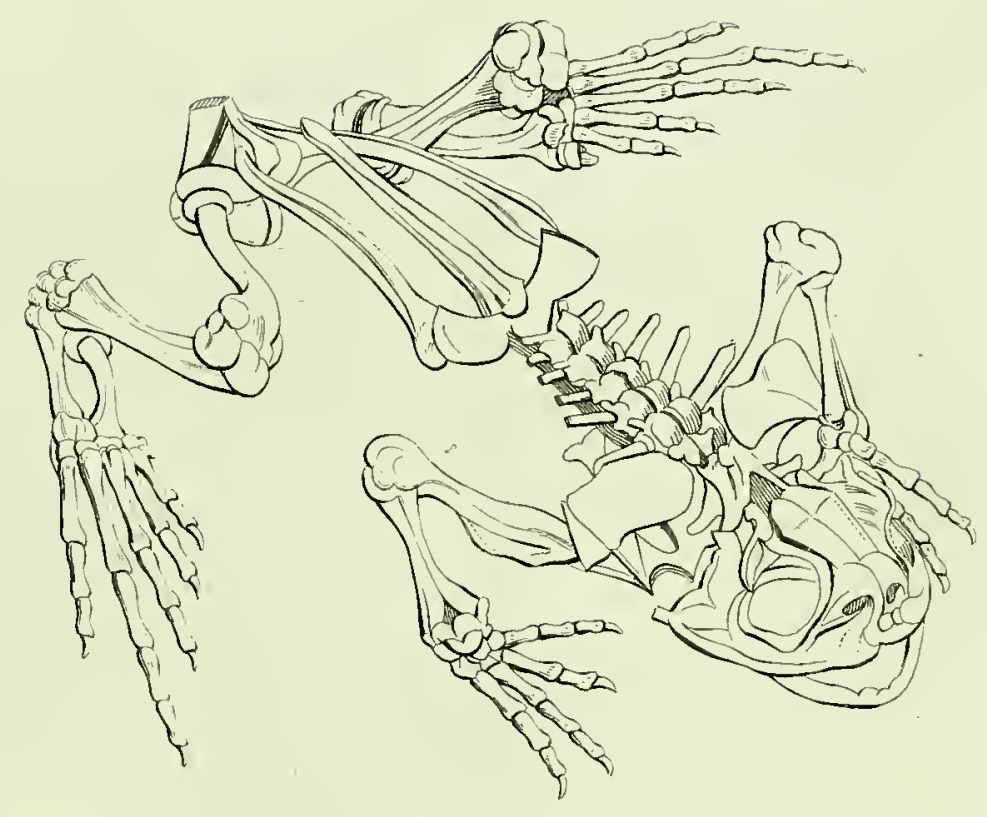





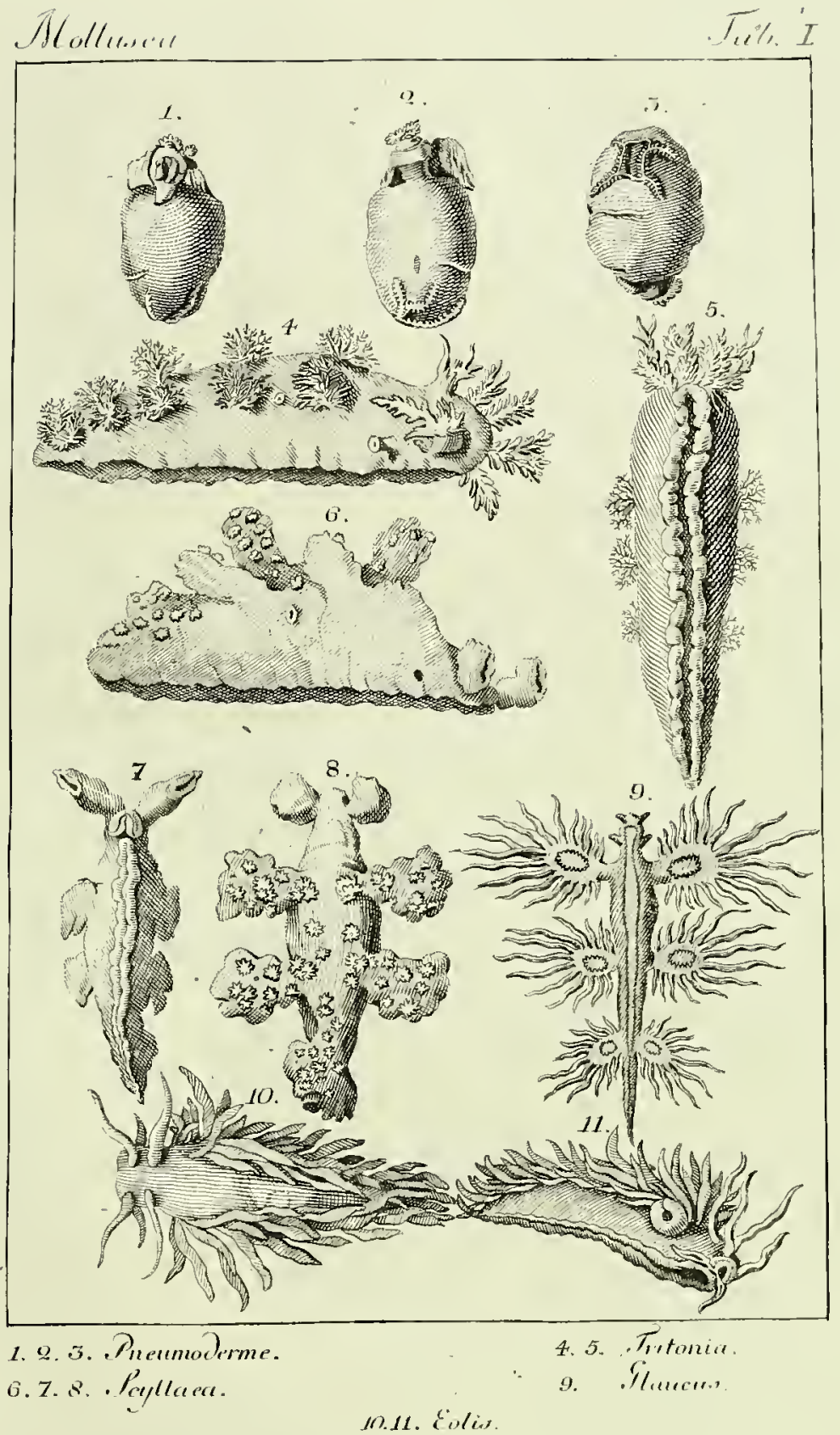



Molleseat.

Tab. II.

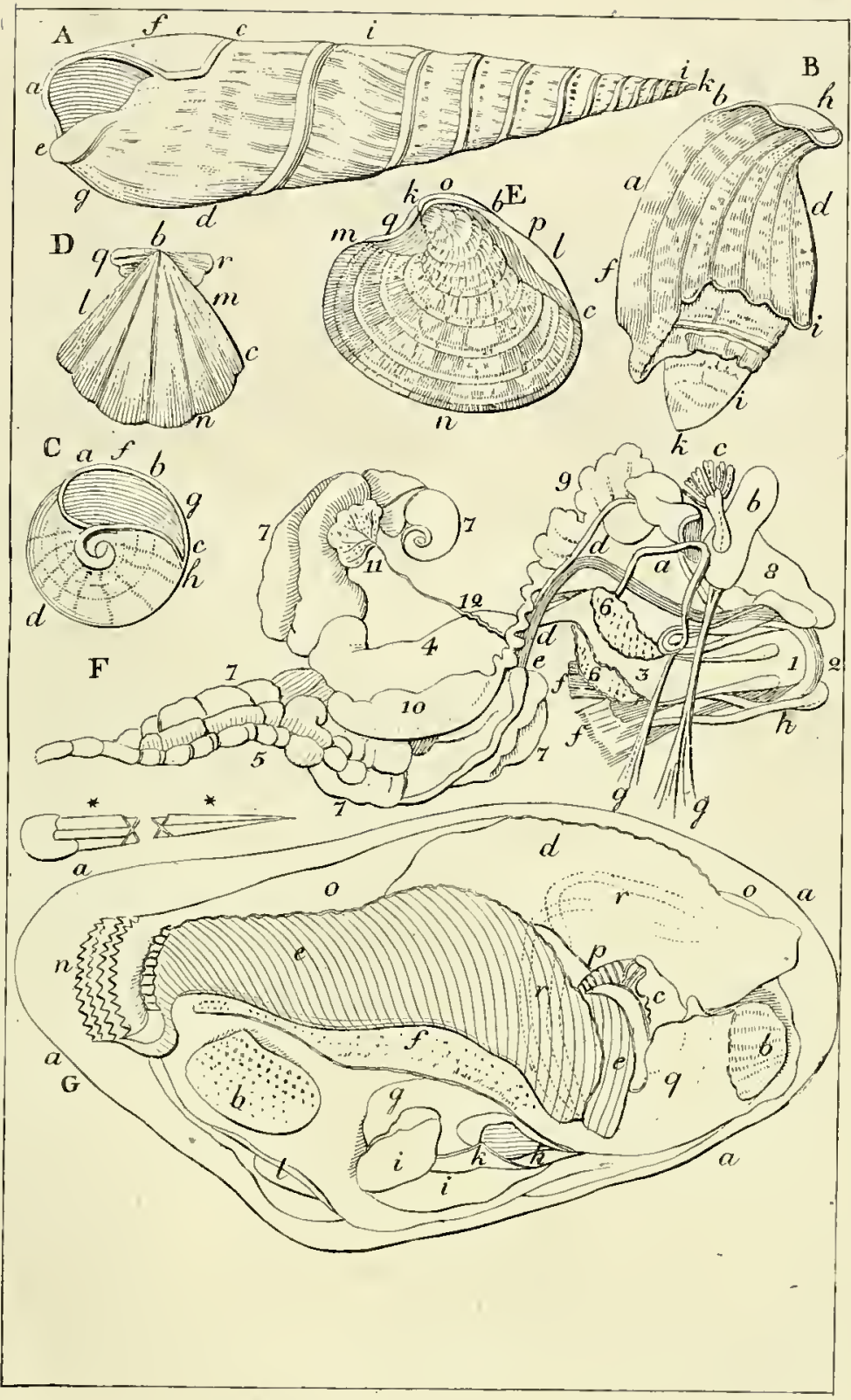




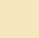




\section{.}




\section{.}


Oardod 

